RHÉTORIQUE DU TEXTE NUMÉRIQUE : FIGURES DE LA

LECTURE, ANTICIPATIONS DE PRATIQUES

Alexandra Saemmer

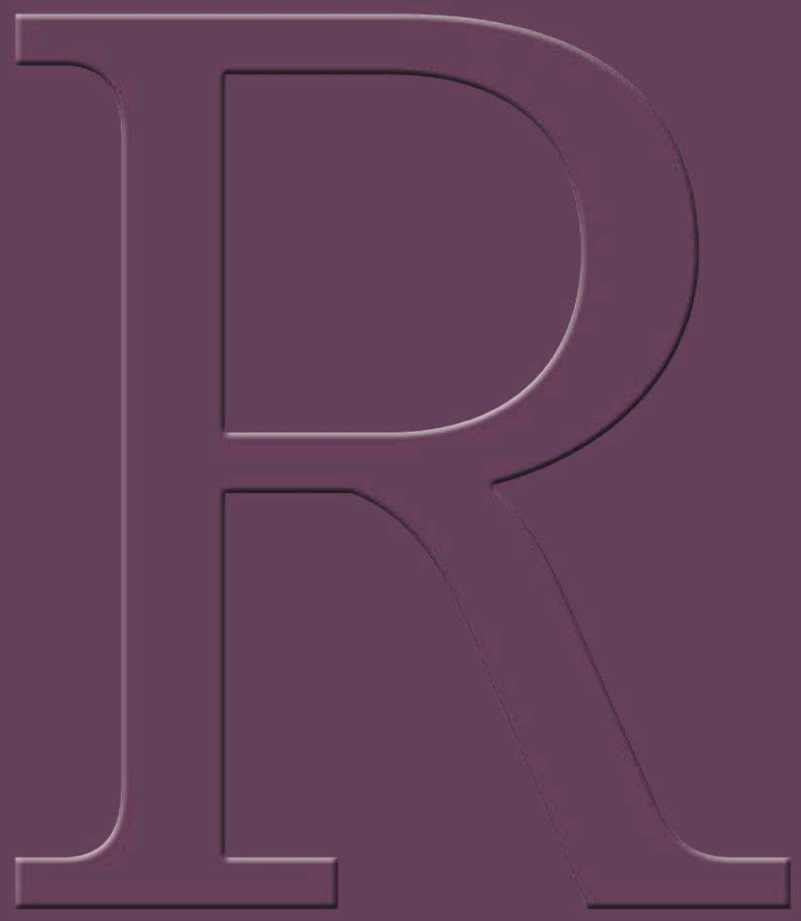




\section{Rhétorique du texte numérique}

Figures de la lecture, anticipations de pratiques

\section{Alexandra Saemmer}

DOI : 10.4000/books.pressesenssib.3870

Éditeur: Presses de l'enssib

Lieu d'édition : Villeurbanne

Année d'édition : 2015

Date de mise en ligne : 21 janvier 2019

Collection : Papiers

ISBN électronique : 9782375460139

\section{Qboooks}

http://books.openedition.org

\section{Édition imprimée}

Date de publication : 1 janvier 2015

ISBN : 9791091281454

Nombre de pages : 276

\section{Référence électronique}

SAEMMER, Alexandra. Rhétorique du texte numérique : Figures de la lecture, anticipations de pratiques. Nouvelle édition [en ligne]. Villeurbanne : Presses de l'enssib, 2015 (généré le 18 février 2021).

Disponible sur Internet : <http://books.openedition.org/pressesenssib/3870>. ISBN : 9782375460139 DOI : https://doi.org/10.4000/books.pressesenssib.3870.

(C) Presses de l'enssib, 2015

Conditions d'utilisation

http://www.openedition.org/6540 


\section{RHÉTORIQUE DU TEXTE NUMÉRIQUE : FIGURES DE LA}

LECTURE, ANTICIPATIONS DE PRATIQUES

Alexandra Saemmer

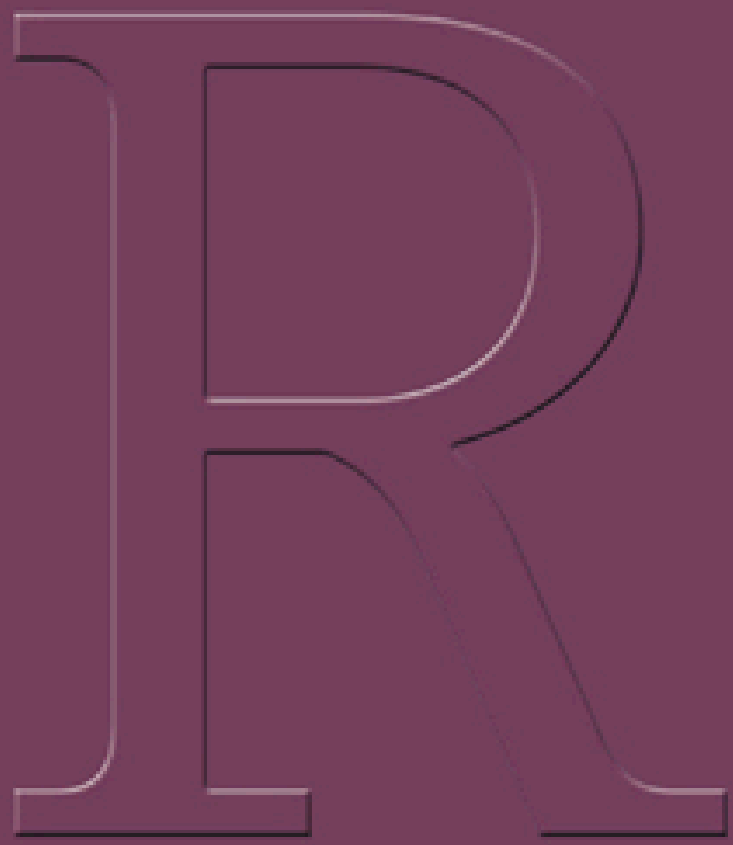


Beaucoup de lectures s'effectueront dans quelques années sur des dispositifs numériques, quels que soient les champs à l'œuvre. La sensibilisation aux spécificités du texte numérique est donc un enjeu important pour la recherche, la lecture et l'écriture.

Cet ouvrage a pour but d'analyser comment le texte préfigure les pratiques, et comment le lecteur y répond en mobilisant ses imaginaires personnels. Il identifie, pour la première fois de façon exhaustive, les procédés rhétoriques de l'hyperlien et de l'animation textuelle, et propose à la fois des outils de défense contre certains effets de manipulation du texte numérique, et des repères pour permettre au lecteur de goûter à un nouveau plaisir du texte.

L'objectif de Rhétorique du texte numérique est de sensibiliser lecteurs, chercheurs, auteurs et éditeurs aux possibles expressifs du texte numérique, et de leur permettre ainsi de mieux connaître et maîtriser ses procédés d'argumentation, de persuasion, de narration, de métaphorisation et de séduction. La connaissance de ce champ des possibles favorisera l'émergence d'une nouvelle culture de l'interprétation, réflexive et critique.

Alexandra Saemmer est professeure des universités en sciences de l'information et de la communication à l'université Paris 8, Centre d'études sur les médias, les technologies et l'internationalisation (CEMTI). Elle est par ailleurs auteure de littérature numérique. 
Alexandra Saemmer est professeure des universités en sciences de l'information et de la communication à l'université Paris 8, Centre d'études sur les médias, les technologies et l'internationalisation (CEMTI).

Rhétorique du texte numérique : figures de la lecture, anticipations de pratiques [Texte imprimé] / Alexandra Saemmer - Villeurbanne : Presses de l'enssib, cop. 2015. - 1 vol. (288 p.) ; 23 cm. (Collection Papiers) ISBN 979-10-91281-45-4. (br.) : $38 €$

Rameau :

Hypertextes

Lecture sur écran

Multimédias interactifs -- Art d'écrire

Livres numériques

Littérature et Internet

Dewey : 028.9 


\section{Remerciements}

Je tiens à exprimer ma vive gratitude à Madjid Ihadjadene, Claude Baltz, Jean-Jacques Boutaud, Bertrand Gervais, Yves Jeanneret, Françoise Paquienséguy et Brigitte Simonnot. Je remercie chaleureusement Claire Bélisle pour son aide précieuse et ses conseils, qui depuis tant d'années m'ouvrent toujours de nouvelles perspectives.

Mes remerciements les plus amicaux vont également à Philippe Bootz, Serge Bouchardon, Annette Cattenat, Jean Clément, Lucile Haute, Monique Maza, Christine Poitevin, Imad Saleh, Fabien Granjon, ainsi qu'à Isabelle Moindrot et Marie-Hélène Tramus pour toutes les belles collaborations au niveau de l'enseignement, de la recherche et de la création à l'université Paris 8.

Merci enfin à ma famille et à ma belle-famille pour leur soutien et leur patience.

À Thomas. 


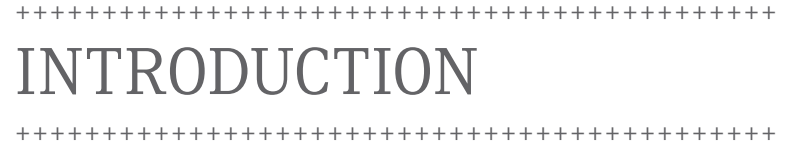




\section{INTRODUCTION}

Il n'y a pas de société d'information sans "cyberculture ». Nous avons besoin d'instruments théoriques appropriés pour penser la complexité et la (dé)cohérence d'ensemble de la société d'information.

Claude Baltz, Documents de séminaire, 2009.

Le sens n'étant pas immanent au texte, mais à ses pratiques d'interprétation, il doit être rapporté à elles.

François Rastier, Arts et sciences du texte, Paris, PUF, 2001, p. 35.

J e numérique fera-t-il disparaître le texte ? Un texte a besoin d'être lu pour exister, et nombreux sont actuellement les discours qui mettent en doute l'existence d'une vraie lecture sur support numérique. Sur Internet notamment, le lecteur ne ferait plus que survoler et cliquer. Ces affirmations s'accompagnent fréquemment de cris d'alarme quant à la disparition de la culture humaniste, de la réflexion critique et de l'intériorité, et frappent par leur caractère déterministe ${ }^{1}$. Ils donnent en effet l'impression que tous les lecteurs pratiquent la lecture de la même façon dès qu'un texte s'affiche sur un écran, peu importent les dispositifs (ordinateur, tablette, téléphone portable ou liseuse), peu importent les éléments partagés entre texte et lecteur (par exemple, des allusions à des événements historiques ou l'évocation de personnages publics supposés connus), peu importent les intentions et motivations individuelles du sujet lisant. La lecture numérique est décrite comme impatiente, rapide et forcément superficielle, au point que certains préfèrent même ne plus parler de lecture. Elle est opposée à la pratique du livre papier présentée comme longue, patiente, concentrée et réflexive ${ }^{2}$. La nostalgie de cette pratique a même inspiré la création d'un parfum imitant l'odeur du livre ${ }^{3}$... Alors que l'Internet regorge de textes, les lecteurs de ces écritures numériques semblent décidément faire défaut.

Les études menées depuis plusieurs décennies par le ministère de la Culture sur les pratiques culturelles des Français confirment certes l'idée

1. Voir par exemple Carr [2008] ; Desrichard [2011] ; Baccino [2011].

2. Desrichard [2011] ; Frommer [2011].

3. Paper Passion, parfum de Gerhard Steidl, Karl Lagerfeld et Geza Schoen. 
d'une diminution constante du nombre de " grands lecteurs $»^{4}$. Or, il faut préciser que la lecture sur dispositif numérique n’a pas encore trouvé sa place dans ces statistiques. D’après The Economist du 27 février 2010, la quantité de textes lus, qui déclinait pendant plusieurs décennies à cause de la télévision, a quasiment triplé depuis 1980 grâce aux textes mis en ligne. En principe, et bien que certains présument le contraire [par exemple, Boullier, 2011, 41], les images animées n’ont donc pas encore remplacé le texte. Nous lisons globalement plus de textes qu'avant. Les données citées par The Economist ne précisent pourtant pas comment nous lisons ces textes sur support numérique. Et c'est en effet plutôt la pratique de lecture numérique, et non pas la quantité incontestable de textes disponibles sur les dispositifs, qui se trouve dans la ligne de mire des critiques.

Pour des auteurs comme Nicholas Carr [2008], le constat est sans appel. Observant sa propre pratique, Carr affirme qu'il lui est désormais difficile de rester concentré sur un seul texte pour en effectuer une lecture approfondie : "Je me sens comme si j'étais sans cesse en train de ramener mon cerveau volage au texte. La lecture profonde qui me venait jadis tout naturellement est maintenant devenue un combat $»^{5}$. Carr s'appuie sur des recherches récentes consacrées à la plasticité du cerveau pour argumenter que ses circuits neuronaux ont été définitivement modifiés par les pratiques de lecture de survol sur support numérique. Pour Carr comme pour beaucoup d'autres critiques de la lecture numérique, dont Susanne Gaschke [2009] qui a consacré à l'« abrutissement numérique » un ouvrage très remarqué en Allemagne, l'« hyper-attention » [déjà diagnostiquée par la critique américaine N. Katherine Hayles en 2007] constitue une impasse pour le texte, devenu désormais un objet de consommation comme un autre.

Thierry Baccino [2011, 63], spécialiste en psychologie cognitive qui a étudié la lecture numérique à l'aide d'enregistrements des mouvements de l'œil, parle de cette pratique comme d'une " pseudo-lecture » : " Pseudo car surfer sur les pages du Web mêlant articles courts, vidéos, audio, animations de toutes sortes n'est pas similaire à une lecture attentive et profonde, que l'on pratique sur un livre imprimé ». Sur un dispositif numérique, le lecteur balaierait rapidement les textes. Son attention serait « imparablement » attirée par d'autres informations apparaissant en simultané. Le Bulletin des bibliothèques de France [numéro 5 de 2011] consacré aux « Métamorphoses de la lecture » réunit un grand nombre

4. Enquêtes sur les «Pratiques culturelles des Français », ministère de la Culture et de la Communication, depuis 1973, [en ligne] : < http://www.pratiquesculturelles.culture.gouv.fr/ >

5. Je reprends ici la traduction effectuée par Vandendorpe [2011, 52]. 
d'articles très critiques envers le potentiel de la lecture numérique. Certains auteurs vont jusqu'à déclarer son impossibilité, se tournant vers le livre comme seul garant d'existence du texte [Desrichard, 2011].

Face à ce déferlement de discours alarmistes, d'autres proposent au contraire une vision optimiste de la lecture numérique. Eux aussi recourent à des études sur le cerveau, mais pour affirmer qu'au lieu de nous rendre " stupides », la lecture numérique stimule de façon particulièrement forte certaines de nos capacités cognitives. Christian Vandendorpe [2011, 53] reprend les recherches du même Thierry Baccino qui, dans le Bulletin des bibliothèques de France, considère la lecture numérique comme une «pseudo-lecture », en interprétant positivement le constat que «la lecture sur écran demande un surcroît de travail au cerveau et même un fonctionnement différent. Les zones de l'encéphale qui contrôlent les prises de décision et les raisonnements complexes sont plus sollicitées que pour une lecture sur papier » [2009, 63]. La rapidité avec laquelle beaucoup de lecteurs parcourent les textes numériques pour en extraire des informations est interprétée par l'auteur non pas comme un signe d'abrutissement, mais comme la preuve d'une concentration accrue.

Christian Vandendorpe [2011, 58] pense donc que l'attention du lecteur est particulièrement en éveil lorsqu'il ne se contente plus de suivre le fil d'un texte mot à mot, mais qu'il évalue la pertinence des éléments textuels en fonction d'une intention de lecture en sélectionnant rapidement les éléments les plus appropriés. Au lieu d'incriminer les industries culturelles de pousser le lecteur vers une " consommation » toujours plus accélérée de (pseudo-)informations qui étouffent le sens critique, des auteurs comme Kevin Kelly [2010], éditeur du magazine américain Wired, observent certes une certaine " fluidité » du texte numérique, mais affirment que celle-ci a provoqué un état d'incertitude tout à fait salutaire, propice à la prise de position critique : "Je suis moins intéressé dans la vérité, avec un grand $\mathrm{V}$, et plus intéressé dans les vérités, au pluriel $»^{6}$.

Dans la plupart des discours alarmistes prédisant la disparition du texte sur les dispositifs numériques, comme dans les discours qui, au contraire, constatent avec optimisme que nous lisons plus et de façon complexifiée, il manque pourtant une donnée essentielle : un examen attentif du texte numérique à lire avec ses spécificités.

Claire Bélisle [2011, 39] et Christian Vandendorpe [2011, 53], parmi d'autres, ont engagé une avancée essentielle dans l'étude de la lecture

6. Je reprends la traduction par Bélisle [2011, 31]. 
numérique en insistant sur l'intention du lecteur : celui-ci ne lit en effet pas un texte numérique de la même façon lorsqu'il doit rapidement disposer d'une information précise, lorsqu'il va sur Internet pour se tenir au courant des actualités politiques, lorsqu'il veut se détendre en feuilletant un magazine people sur le Web ou qu'il cherche à se perdre dans des univers multimédias plaisants à l'œil et agréables au toucher.

Il paraît également important de rappeler que le texte lui-même entre de diverses manières en résonance avec les attentes individuelles et socialement partagées du lecteur. Autrement dit, le lecteur ne lit certainement pas avec la même attention un texte numérisé de Hegel, un article de parismatch.fr, un fragment autobiographique posté par un ami sur Facebook ou une hyperfiction, car ces textes ne sollicitent pas son attention de la même manière et ne mobilisent pas les mêmes contenus, stratégies et procédés rhétoriques.

L'existence d'une seule lecture papier, concentrée et réflexive, ne relèverait-elle pas du mythe ? Il arrive à tout lecteur de feuilleter un livre, de parcourir le sommaire sans s'engager dans une découverte approfondie des contenus annoncés. Certains genres textuels comme l'article encyclopédique se prêtent même tout particulièrement à cette pratique en proposant des contenus, en mobilisant des procédés rhétoriques et présentations visuelles spécifiques.

L'existence d'une seule lecture numérique, rapide et superficielle, ne résiste pas non plus à l'examen des pratiques. Il arrive sans doute à tout lecteur de s'attarder sur un texte édité sur support numérique, de le lire du début jusqu'à la fin et de prendre le temps de réfléchir sur ce qu'il a lu : pratique de lecture qui s'enracine d'une part dans la motivation du lecteur et - telle sera ma principale hypothèse - qui, d'autre part, est anticipée par le texte lui-même, par ses contenus, ses stratégies et procédés rhétoriques, ainsi que par sa matérialité sur la page-écran. L'objectif de mon livre sera ainsi de cerner les pratiques de lecture modélisées par le texte numérique, à travers ses procédés rhétoriques et ses formes graphiques sur la page-écran.

Ma démarche s'inscrit dans la filiation de la "rhétorique nouvelle » [Perelman, 2000] et des théories de la réception [en outre Iser, 1976 ; Jauss, 1972-1978] tout en prenant en compte les « matérialités » de la communication. Des outils d'analyse rhétoriques et sémiotiques me permettront de cerner avec la nécessaire précision le « potentiel d'action » du texte numérique. Cette notion, empruntée à Wolfgang Iser [1976], montre cependant que ces outils ne sont pas mobilisés pour faire émerger une signification immanente, stable et transhistorique du texte numérique. Il s'agit, plus modestement, 
de circonscrire ce que le texte numérique met à disposition du lecteur, qui actualise ensuite ce potentiel à travers ses pratiques personnelles.

L'étude du potentiel d'action du texte constitue un lieu de croisement fort entre les théories de la réception venant des sciences du texte, et les sciences de la communication. Mon apport consiste à mettre en œuvre cette convergence sous forme d'une rhétorique spécifique que j'ai décidé d'appeler « rhétorique de la réception ». Qu'est-ce que j'entends par ce terme?

Tout texte préfigure son lecteur et ses pratiques à travers ses contenus et ses procédés rhétoriques. La rhétorique de la réception identifie et analyse ces préfigurations qui sont alors appelées " figures de la lecture ». Je partirai de l'hypothèse que l'hyperlien et l'animation constituent des éléments fondamentaux du texte numérique, et qu'ils mobilisent des figures spécifiques qui seront identifiées tout au long du livre. Dans le cas de l'hyperlien, nous verrons que ces figures modélisent les pratiques du lecteur, notamment à travers le rapport de contiguïté, temporelle ou causale, entre deux ou plusieurs textes reliés par des mots cliquables (pensons à l'exemple d'un mot relié par hyperlien à sa définition), ainsi qu'à travers le couplage entre le texte et des enchaînements de gestes (prenons le fameux geste du clic effectué sur un hyperlien). Dans le texte animé, ces figures sont fondées sur le couplage entre un texte et du mouvement (par exemple, lorsqu'un mot clignote). Le mouvement et le geste font partie des spécificités formelles du texte numérique. La police de caractère et la couleur ou la taille des lettres font également partie des éléments formels du texte, mais ne sont bien sûr pas spécifiques au texte numérique. Toutes les formes qui entourent le texte numérique sur une page-écran, sans être elles-mêmes du texte, préfigurent également les pratiques du lecteur : en font partie les couleurs du fond (imitant parfois la page papier), la mise en espace $d u$ texte (par exemple, sous forme de tableau), bref toutes ces matérialités de la communication qui mettent littéralement le texte en forme.

Ces formes et figures du texte numérique sont actualisées de façon forcément incomplète dans une situation de lecture précise. Loin de ne suivre que les anticipations faites par le texte et ses matérialités, mais loin aussi de s'adonner seulement à des motivations individuelles, le lecteur s'inscrit dans des " communautés interprétatives » [Fish, 1980], au sein desquelles se partagent des référents culturels, habitudes et attentes.

La rhétorique de la réception du texte numérique repose donc non seulement sur l'examen du potentiel d'action du texte numérique avec ses matérialités (voir le chapitre III). Les imaginaires socialement partagés du dispositif, de l'Internet et du texte numériques jouent un rôle indéniable 
dans le processus de conception et de lecture, et doivent être circonscrits en amont de l'analyse du potentiel d'action du texte numérique et orienter celle-ci (voir le chapitre II). Pour le dire dans des termes empruntés à la sémiotique piercienne, l'analyse du texte numérique ne peut se faire sans prise en compte de l'« interprétant », étape qui s'appuie ici en outre sur des études empiriques de nature quantitative et qualitative par observation, questionnaire et entretien. La rhétorique de la réception circonscrit ainsi le potentiel d'action du texte numérique en fonction des champs d'intersection possibles entre les anticipations des pratiques de lecture par les formes et figures du texte, et les actualisations de ces anticipations par le lecteur en fonction de son « horizon d'attente » [Jauss, 1972-1978; 2010].

La rhétorique a toujours proposé, par le biais de ses procédés, une restructuration du réel adressée à un public. La rhétorique du texte numérique développée ici est à la fois une science du texte en tant qu'elle étudie celui-ci comme matière potentiellement signifiante, et une science de ses lectures en tant qu'elle se penche sur des pratiques individuelles et socialement partagées de cette restructuration du réel.

Il me semble que la nécessité de remettre le texte au centre des réflexions sur la lecture numérique émerge tout particulièrement dans les polémiques autour de l'une de ses spécificités les plus prégnantes, l'hyperlien. Même si l'animation textuelle est étudiée dans ce livre comme l'une des caractéristiques importantes du texte numérique, l'hyperlien est scruté avec une attention appuyée. Ce retour à l'hyperlien, dont certains n’ont pas hésité à déclarer la « mort », doit être explicité.

Je précise d'abord que j'utiliserai le terme « hyperlien » dans le sens d'élément textuel " hyperlié » à lire et à manipuler, qui est inséré dans un texte (appelé " texte géniteur ») et renvoie vers un texte généralement encore invisible (appelé " texte relié »). Ma définition s'inspire de celle de l'hyperlien comme " signe passeur » [Jeanneret, Souchier, 1998] qui met en relation les dimensions de " signe lu », de " signe interprété » et d'« outil manipulable ». L'hyperlien dans ce sens large est omniprésent dans le texte numérique : dans les résultats proposés par les moteurs de recherche, les journaux en ligne, les portails d'information et les sites commerciaux, les réseaux sociaux, la littérature numérique et le jeu vidéo. Le terme " hypertexte ", également mobilisé dans ce travail, renvoie plutôt à un texte parsemé d'hyperliens et relié à d'autres textes, donc à un

7. Par exemple Dylan Kinnett, “The Death of Hypertext?", 2012, [en ligne] : < http://nocategories. net/ephemera/the-death-of-hypertext/ >. 
ensemble textuel plus étendu « contenant des nœuds liés entre eux par des hyperliens » (définition canonique reprise en outre par Wikipédia) ${ }^{8}$.

Pour Thierry Baccino, l'hypertexte est fondé sur une idée « louable », mais problématique dans son actualisation lors de la lecture : celle de rendre disponible, par le biais d'hyperliens, « toutes les informations associées de près ou de loin à une notion » [2011, 64]. Dans la pratique, la « multiplicité de niveaux » provoquerait souvent « une perte de l'objectif initial de lecture ». La cohérence, " propriété essentielle de la compréhension », ne pourrait plus être établie entre les différents passages lus : en résulterait une « désorientation » du lecteur. Cette observation, sans doute valide dans certaines situations de lecture, se trouve généralisée par l'auteur. Or, certains textes parsemés d'hyperliens offrent au lecteur des aides d'orientation : ils peuvent être porteurs d'annonces, qui anticipent sur ce qui va suivre lorsque le lecteur aura cliqué (prenons l'exemple suivant relevé dans un journal en ligne : « cliquez ici pour découvrir la chronique des événements »).

M'éloignant des définitions purement techniques, je considère donc l'hyperlien comme la trace d'une interprétation effectuée par un Auteur, qui se trouve plus ou moins explicitée dans le texte géniteur contenant l'hyperlien. J'affecte le terme "Auteur » d'une majuscule pour indiquer que l'auteur n'est pas toujours celui du texte géniteur (dans certains journaux en ligne, des rédacteurs spécialisés insèrent les liens), et qu'il peut même être une machine (dans le cas des hyperliens promotionnels automatiquement insérés, par exemple). Quoi qu'il en soit de son identité, l'Auteur lie donc un texte à un autre parce qu'il considère qu'ils doivent être reliés, pour différentes raisons que la rhétorique du texte numérique s'efforce de cerner. Bien sûr, il faut néanmoins garder à l'esprit que le potentiel d'action d'un hyperlien dépasse cette intention de l'Auteur : celui-ci n'est jamais maître de toutes les réceptions possibles de son texte. Voilà pourquoi il peut certes s'avérer utile de recourir à des entretiens avec des auteurs et concepteurs pour cerner leurs intentions quand ils insèrent un hyperlien, sans pour autant prétendre que ces déclarations donnent accès au vrai sens du texte numérique.

Je récapitule. D’une part, l'Auteur anticipe sur les pratiques de lecture en liant deux textes par un hyperlien ; d'autre part, le lecteur approche cet hyperlien avec plusieurs attentes, dont certaines sont socialement

8. «Un système hypertexte est un système contenant des nœuds liés entre eux par des hyperliens permettant de passer automatiquement d'un nœud à un autre. Un document hypertexte est donc un document qui contient des hyperliens et des nœuds. », Wikipédia, [en ligne] : < http:// fr.wikipedia.org/wiki/Hypertexte >. 
partagées : l'hyperlien semble majoritairement approché par le lecteur avec l'espoir qu'il livre des réponses factuelles, des compléments d'information, des preuves réduisant l'incertitude du sujet sur le monde ${ }^{9}$. La rhétorique de l'hyperlien mise en œuvre dans ce livre montrera, études de corpus à l'appui, que cette présomption d'information n'est pas toujours satisfaite. Dans les corpus journalistique, littéraire et publicitaire étudiés au chapitre III, l'hyperlien relie parfois des textes porteurs de points de vue divergents, ou provoque même des renversements ironiques entre texte géniteur et texte relié. Il anticipe donc sur des pratiques de lecture diversifiées, dont la lecture attentive, alors que la lecture numérique est souvent décrite comme exclusivement rapide et superficielle. Une partie de la fameuse désorientation du lecteur numérique s'explique sans doute par cette divergence entre la modélisation des pratiques par le texte numérique, et les attentes partagées par grand nombre de lecteurs.

En circonscrivant avec précision les multiples figures de la lecture du texte numérique, la rhétorique de la réception essaie alors de démontrer la nécessité d'une complexification des pratiques. La connaissance du champ des possibles de la rhétorique du texte numérique pourrait ainsi devenir un élément fondateur pour une nouvelle culture de l'interprétation : un art de lire qui aura comme but de mieux appréhender les enjeux communicationnels du texte numérique. Une telle culture de l'interprétation, réflexive et critique, du texte numérique sera mise en œuvre tout au long du chapitre III.

Cette culture de l'interprétation est aussi inspirée par l'évolution de mes propres pratiques de lecture numériques. À l'instar de Nicholas Carr [2008] ou plus académiquement de Marcel Prous ${ }^{10}$, je m'autorise une brève remarque sur ces pratiques : alors qu'elles étaient, il y a une dizaine d'années encore, marquées par une désorientation certaine, je les sais aujourd'hui fortement diversifiées. Parfois je parcours rapidement de grandes quantités de textes à la recherche d'une information précise, guettant la perche qui me fera avancer; parfois je m'adonne sans retenue au plaisir du clic voyeur ; parfois je divague avec délectation, parfois je regrette d'y perdre mon temps ; parfois il m'arrive de passer quatre ou cinq heures d'affilée à la lecture d'un texte scientifique avec hyperliens ou d'une hyperfiction. Cette dernière pratique, qu'elle soit réflexive ou immersive, est longue et attentive aux détails. Je l'ai apprise progressivement. Le

9. Voir par exemple Charney [1994] ; Nielsen [1999] ; Tosca Parajes [2000] ; Ensslin [2007] ; Boucher [2011] et études empiriques citées plus loin.

10. «Qui ne se souvient comme moi de ces lectures faites au temps des vacances... » [Proust, 1993, 8]. 
repérage des formes et figures du texte numérique a joué un rôle essentiel dans cet apprentissage. Une prise de conscience de l'horizon d'attente personnel et socialement partagé ayant forgé mes expectatives et habitudes sur les dispositifs numériques y a également contribué.

Je ne suis pas la seule lectrice de textes numériques reconvertie à la patience, tant s'en faut. Au Japon, les fictions pour téléphones portables se dévorent depuis plusieurs années [voir article "Cell Phone Novels Come of Age" sur Japantoday ${ }^{11}$. Sur Facebook, force est de constater que les usagers s'entrelisent parfois avec attention, car ils se répondent de façon pertinente. Dans le champ de recherche consacré à la littérature numérique, après deux décennies marquées par des discours généralistes et des analyses orientées « dispositif », rares sont aujourd'hui les appels à articles qui ne sollicitent pas des close readings, définis comme analyses détaillées des particularités dispositives et textuelles d'une œuvre littéraire numérique $^{12}$. Après la disparition déclarée du texte à lire, parfois saluée comme échappée finale des prisons du discours linéaire et de la domination du logos [Bolter, 1991], parfois redoutée comme symptôme du déclin des humanités et des cultures de l'interprétation [Birkerts, 1994 ; Desrichard, 2011], il s'agit dans ce livre de proposer des outils pour adopter une attitude nuancée, à la fois ouverte et critique, envers le texte numérique.

Science du texte et de ses pratiques, la rhétorique du texte numérique ne s'adresse pas qu'au lecteur. Quelles significations peut-on faire émerger en reliant deux ou plusieurs textes par des hyperliens ? Peut-on argumenter avec l'hyperlien, peut-on raconter une histoire ? Où place-t-on les hyperliens pour orienter les attentes et pratiques du lecteur dans tel ou tel sens ? Quel est le potentiel de persuasion d'une animation textuelle? Voilà des questions auxquelles tout praticien de l'écriture numérique est confronté. La rhétorique du texte numérique propose des outils d'analyse et des typologies avec la conviction qu'une connaissance des possibles du texte numérique aide le lecteur et l'auteur à mieux appréhender le texte numérique, que ce soit dans un contexte universitaire, scolaire, public, privé, professionnel ou de loisir.

Le dispositif de lecture occupe une place importante dans cette rhétorique en tant qu'il conditionne l'apparition du texte à l'écran puis sa

11. Patrick W. Galbraith, “Cell Phone Novels Come of Age”, 2009, [en ligne] : < http://www.japantoday.com/category/arts-culture/view/cell-phone-novels-come-of-age >.

12. Voir, par exemple, l'appel à participation pour le congrès de la Electronic Literature Organization 2010, [en ligne] : < http://www.brown.edu/Conference/Electronic_Literature_Organization/ call.php >. 
disparition, de façon plus ou moins prévisible par l'Auteur. En effet, le texte numérique n'est pas solidaire avec son dispositif : il peut migrer de l'ordinateur fixe vers le téléphone mobile, il s'actualise dans différents systèmes d'exploitation, à différentes vitesses de calcul et selon des réglages parfois très divergents. Cette " labilité » du dispositif [Bootz, dès 1990] doit être prise en compte dans une rhétorique qui analyse les figures du texte numérique comme des traces et anticipations de lectures.

En résumé, l'objectif de ce livre est donc l'élaboration d'une rhétorique de la réception du texte numérique - couplage de termes qui peut paraître paradoxal si l'on se tient à une définition de la rhétorique comme simple ornement détachable du contenu, et qui énonce pourtant exactement de quoi il s'agit dès que l'on considère le contenu, la forme et la réception d'un texte comme intimement liés. Étudier les figures de la lecture du texte numérique non pas comme des procédés rhétoriques signifiants par eux-mêmes, mais comme des potentiels anticipant sur des pratiques de lecture, voilà l'ambition de cette rhétorique qui emprunte des concepts fondateurs aux approches pragmatiques du texte [Gervais, 1998 ; 2007], aux analyses sémio-pragmatiques du dispositif [Souchier, 1998 ; Jeanneret, Souchier, 1998, 2005 ; Jeanneret, 2008] et à la « sémiotique du sensible » [Boutaud, 2007; Landowski, 2004] tout en proposant une typologie inédite des figures et formes spécifiques au texte numérique.

J'insiste d'emblée sur le fait que cette typologie de figures, malgré son caractère opérationnel dans beaucoup de champs de l'écriture numérique comme le journalisme, la littérature, la publicité, le blog, le livre numérique enrichi ou les réseaux sociaux, n'est pas conçue comme une liste de catégories prêtes à l'emploi. L'identification des figures du texte numérique ne peut se faire qu'en prenant en compte le genre textuel, le contexte, les spécificités du dispositif, tout comme les attentes et imaginaires qui constituent l'horizon d'attente du lecteur.

C'est ainsi que la rhétorique de la réception représente une nouvelle approche au carrefour des méthodologies, qui recourt à l'analyse rhétorique et sémiotique comme à l'étude empirique des pratiques de lecture par questionnaire, entretien ou observation, pour faire émerger une approche critique du texte numérique dans ses contextes d'émergence culturels et communicationnels.

Les fondements théoriques de la rhétorique de la réception seront présentés au chapitre I. Des emprunts seront faits à la rhétorique (traditionnelle et nouvelle), science de décryptage du texte et de ses pratiques. Les théories de la réception aideront à circonscrire, d'abord de 
façon théorique, ce qui constitue le potentiel d'action du texte en relation avec l'horizon d'attente du lecteur. Des approches sémio-pragmatiques des matérialités de la communication fourniront des conceptualisations et vocabulaires utiles pour étudier le texte dans son contexte d'énonciation éditoriale, actualisé dans un dispositif technique. Plus précisément, il s'agira d'expliciter l'articulation entre les préfigurations de pratiques par deux particularités du texte numérique, l'hyperlien et l'animation textuelle (introduites dès le début du chapitre afin de plonger le lecteur in medias res), avec les imaginaires, attentes et habitudes du lecteur circonscrits à l'aide de méthodologies empruntées aux sciences sociales. Bref, j'expliquerai dans ce premier chapitre comment la prise en compte de l'interprétant se place au cœur de la rhétorique de la réception.

Dans les chapitres II et III, cette méthodologie sera mise en œuvre à partir de l'analyse de trois corpus, journalistique, narratif et publicitaire. Comme annoncé au chapitre I, les imaginaires, habitudes et attentes du lecteur contemporain vis-à-vis du dispositif, de la lecture numérique, de l'hyperlien, de l'animation et des genres textuels doivent d'abord être sondés avec le plus de précision possible. Après cette étape de circonscription de l'horizon d'attente au chapitre II, le potentiel d'action du texte numérique peut être analysé à partir de corpus. Des typologies de figures modélisant les pratiques de lecture à travers des procédés rhétoriques peuvent être établies.

Le chapitre III est donc consacré à l'identification des figures de la lecture du texte numérique dans trois corpus, journalistique, littéraire et publicitaire. Autrement dit, j'analyserai dans cette partie comment le texte numérique modélise ses pratiques de lecture à travers des formes et des figures de rhétorique qui lui sont propres, en anticipant sur l'horizon d'attente de ses lecteurs potentiels. Je montrerai que ces préfigurations de pratiques ne répondent pas toujours aux imaginaires et attentes les plus courants, notamment informationnels, face au texte numérique : dans le contexte journalistique, par exemple, l'objectif poursuivi semble parfois être plutôt la mise en dialogue de plusieurs points de vue, reflétant la complexité d'une situation ; parfois le jeu avec les attentes du lecteur penche même vers la manipulation fallacieuse, mise au profit de structures de domination médiatico-politiques.

Se dessinera ainsi la nécessité d'une culture de l'interprétation fondée sur un décryptage critique des enjeux communicationnels du texte numérique, qui ne néglige pas pour autant la notion de plaisir de la lecture. 


\section{CHAPITRE I FONDEMENTS THÉORIQUES D'UNE RHÉTORIQUE DU TEXTE NUMÉRIQUE}




\section{FONDEMENTS THÉORIQUES D'UNE RHÉTORIQUE DU TEXTE NUMÉRIQUE}

\section{DEUX CARACTÉRISTIQUES FONDAMENTALES DU TEXTE NUMÉRIQUE}

\section{L'hyperlien, cette essence du Web}

Toute situation réelle est entourée d'un tissu de potentialités. Avant d'être activé, l'hyperlien offre une richesse comparable. L'hyperlien est omniprésent dans le numérique dès qu'on élargit sa définition. Même si le code informatique d'un hyperlien posé sur un nom d'utilisateur de Facebook est, d'un point de vue technologique, fort différent de celui d'un hyperlien par lequel un journaliste de lexpress.fr marque, par exemple, le nom d'une personnalité, le lecteur ne percevra pas cette différence de fonctionnement lors de l'activation. L'hyperlien qui m'intéresse ici est une entité textuelle manipulable qui cache son jeu : le texte relié n'apparaît qu'une fois le geste de manipulation effectué. Ce geste de manipulation n'est pas toujours un clic : le lecteur peut parfois faire apparaître « l'autre texte », par simple effleurement d'un écran tactile, par un geste de grattage... L'un des points essentiels dans ma définition élargie de l'hyperlien est que l'autre texte, appelé « texte relié », a besoin de l'intervention physique du lecteur pour advenir sur l'écran.

Certes, tout texte, en cours de lecture, suscite l'envie d'en découvrir la suite. Un texte papier impose pourtant immédiatement son volume. Le lecteur sait qu'une suite est là, matériellement, parce qu'il perçoit les lettres et paragraphes qui lui restent à parcourir. Confronté à un hyperlien, le lecteur doit prendre le risque de la manipulation pour savoir si l'autre texte existe. 
On peut tourner une page papier à moitié pour jeter un œil sur la suite du texte. On ne peut guère activer un hyperlien à moitié1.

Comme toute lecture, celle d'un hypertexte s'inscrit dans une temporalité. La définition canonique de l'hypertexte en tant que « système contenant des nœuds liés entre eux par des hyperliens permettant de passer automatiquement d'un nœud à un autre $»^{2}$ ne tient pas assez compte de cette dimension fondamentale. Les graphes et cartes de navigation qui ont connu leur heure de gloire autour des années 2000 rassurent éventuellement le lecteur sur l'existence des pages-écran qu'il lui reste à explorer ; en même temps, ils dénaturent l'expérience temporelle de l'hyperlien fondée sur un avant et un après, groupés autour d'un geste de manipulation qui peut être fatidique. Un texte n'est pas qu'un document. Un hypertexte n'est pas qu'un texte avec des liens.

L'hyperlien est une particularité fondamentale du texte numérique. Beaucoup a été dit sur l'hyperlien dont l'invention est attribuée à Theodor Nelson, et qui a été expérimenté par Douglas Engelbart. Considéré d'abord comme outil emblématique d'une nouvelle façon de relier des documents, il a été investi dans les années 1990 de toutes sortes d'espoirs et de fantasmes. Certains auteurs ont mobilisé les métaphores du " rhizome » décentralisé et infiniment proliférant [Landow, 1997, 4] et du réseau neurologique [Zinna, 2002, 3] pour rêver à l'émergence d'une intelligence collective, d'un espace public ouvert à tous, d'une nouvelle plasticité et matérialité du texte, d'une mise à mal de la linéarité, voire d'une échappée définitive du sujet face à l'autorité du texte et du logos dominateur (voir chapitre II pour plus de précisions sur ces imaginaires). L'hyperlien est un puissant générateur d'imaginaires parce qu'il cache si bien son jeu : avant de l'activer, le lecteur ne sait pas ce qui l'attend. Après l'avoir activé, il constate certes que la plupart des hyperliens relient invariablement un texte à un autre. Le texte géniteur contenant l'hyperlien a pourtant souvent, de façon au moins transitoire, disparu de l'écran. C'est ainsi que l'hyperlien met doublement en jeu nos attentes, avant et après son activation.

L'hypertexte " papier », dont l'existence a été proclamée par certains auteurs travaillant sur les filiations entre littératures d'avant-garde et littérature numérique [Landow, 1997], est un mythe parce que le papier

1. Les hyperliens sur le réseau social viadeo.com jouent de façon astucieuse avec cette impossibilité : lorsque le lecteur veut consulter par clic le profil d'un utilisateur, celui-ci apparaît pour quelques secondes, montrant juste assez de volume textuel pour aiguiser la curiosité du lecteur, sans lui permettre pour autant d'entamer la lecture des différentes lignes du profil. S'affiche rapidement, à la place du profil, un formulaire de contact invitant le lecteur à s'inscrire au réseau.

2. Voir note 8, p. 16. 
imposera toujours son volume à la main du lecteur, même si la page a été tournée. Ce n'est pas le cas pour le texte géniteur, celui dans lequel le lecteur active un hyperlien, car ce texte a souvent disparu après l'effectuation du clic ; ce n'est pas le cas non plus pour le texte relié avant son activation.

En effet, l'hyperlien est un puissant générateur d'imaginaires. D'un point de vue fonctionnel, son rôle dans le texte numérique a pourtant toujours semblé aller de soi : il relie des documents. Sa manipulation est d'une grande facilité et ne demande aucun apprentissage particulier. Il suffit d'agir sur une zone interactive. Phénomène récurrent dans notre environnement médiatique, l'hyperlien est devenu si trivial qu'il passe quasiment inaperçu. Lorsque nous avons l'impression que tout a été dit sur un phénomène dans notre entourage et qu'il ne nous surprendra plus, il est grand temps d'interroger le bien-fondé de cette apparente évidence.

\section{L'hyperlien n'est pas qu'une métonymie}

J'ai été d'abord confrontée à l'hyperlien dans le contexte des " humanités numériques » qui commençaient à émerger timidement en France dans les années 1990. À l'époque, je travaillais sur ma thèse de doctorat portant sur un cas d'intertextualité complexe : les traces que la lecture du roman L'homme sans qualités de Robert Musil a laissées dans l'œuvre tardive de Marguerite Duras. À l'instar de ses premiers théoriciens, George P. Landow [1997] et Jay David Bolter [1991], je considérais l'hyperlien comme un outil rêvé pour visualiser ces traces. Alors que j'avais esquissé dans l'introduction de ma thèse (parue en 2002) l'idée que des termes ou phrases hyperliés pourraient permettre de littéralement creuser les mots d'Agatha de Marguerite Duras pour découvrir le sous-texte de L'homme sans qualités de Robert Musil, j'ai continué à visualiser ces traces par la voie traditionnelle du «tableau synoptique».

Je me rassurais de mon manque d'audace en observant l'émergence des " graphes de sites » et autres cartes de navigation à la même époque. Eux aussi permettaient de visualiser de façon synoptique ce qui me paraissait, à ce moment, constituer l'essentiel de l'hypertexte : le fait de relier plusieurs textes de manière complexe. Mes premières analyses de sites débutaient alors systématiquement par le dessin d'une carte de navigation. La possibilité de circonscrire le périmètre d'un site et de montrer comment ses " fragments " sont reliés entre eux s'imposait à la fois comme enjeu de recherche et comme aide à la lecture. Plus les relations entre les fragments étaient multiples et entrecroisées, plus le 
site me semblait innovant par rapport à mon modèle de référence, le livre papier. Pourtant, les graphes les plus précis ne me permettaient pas de faire émerger pourquoi certains textes se trouvaient ainsi reliés.

Est-il plus opérationnel d'évaluer cette relation en mettant en perspective les contenus des textes reliés ? L'hyperlien a parfois été comparé à la figure rhétorique de la métonymie [Manovich, 2001, 228]. Le mot ou l'image hypertextualisés n'annoncent-ils pas en effet, la plupart du temps, une totalité, une explication, un complément d'information ? Il suffit de reprendre l'exemple d'un mot hyperlié à sa définition pour être séduit par cette hypothèse. L'idée de recourir aux figures de la rhétorique papier pour circonscrire les spécificités du texte numérique semblait en tout cas prometteuse.

Pourtant, je suis aujourd'hui persuadée que cette mobilisation de la rhétorique papier pour l'analyse du texte numérique doit être faite avec précaution. Une expression métonymique classique comme « je bois un verre » fait rêver à mille liquides buvables. Un hyperlien « métonymique » posé sur l'énoncé « je bois un verre » relie, suivant l'idée de Lev Manovich, le verre à un contenu liquide : par exemple, à un texte précisant que le verre est rempli de champagne. D'une certaine façon, cette relation abolit le rêve des potentialités caractéristique de la métonymie classique, même si chaque lecteur a un autre goût en bouche en lisant dans le texte relié que le verre est rempli de champagne. Le potentiel métonymique d'un tel hyperlien réside donc moins dans la relation effective établie entre le verre et son contenu liquide que dans les attentes suscitées par le mot « verre » hyperlié avant qu'il soit activé.

La métonymie papier tire son charme du fait d'être annonce de totalité. L'hyperlien informatique peut relier une métonymie à son explication, suivant le vieil adage « qu'est-ce que l'auteur voulait dire par cette métonymie ?». On peut ainsi avoir l'impression que tout hyperlien textuel n'est qu'une expression chiffrée, qu'il suffira d'activer pour savoir de quoi elle parle réellement.

Bertrand Gervais [2006] a formulé cet imaginaire de façon radicale en rapprochant l'hyperlien du fonctionnement d'un signe linguistique qui signifierait tout seul : il renverrait à quelque chose d'autre pour quelqu'un, sauf qu'il le ferait toujours de façon identique une fois l'hyperlien programmé. Si l'on poursuit cette idée, l'hyperlien instrumentaliserait une relation enfin stabilisée entre signifiant et signifié. La stabilité technologique de l'hyperlien suggérerait l'élimination de " l'interprétant » cher à Pierce : le lien et son renvoi existeraient sans jamais varier, indépendamment du lecteur qui choisirait de l'activer et de lire les textes reliés. C'est 
précisément pourquoi l'hyperlien nous place, selon Gervais [2006], dans une logique de la révélation et de l'apparition de vérités.

Cet imaginaire ne résiste évidemment pas toujours à la confrontation aux textes que nous lisons quotidiennement sur les supports numériques. Un mot ou une image hyperliés ne se trouvent pas systématiquement confirmés, expliqués, précisés ou définis par le texte relié : dans la presse en ligne notamment, l'hyperlien associe parfois un argument à son contre-argument, un point de vue à un autre point de vue, une affirmation à son démenti. Tout un éventail de possibilités se déploie entre les pôles de la confirmation du texte géniteur par le texte relié, et leur antagonisme complet.

Imaginons par exemple que le texte relié à l'hyperlien « verre » dans l'énoncé « je bois un verre » renvoie non pas à une illustration du contenu liquide de ce verre, mais à la description d'un champ de fleurs sous un ciel d'été. Quel rapport existe-t-il entre le « verre » et le champ de fleurs ? L'auteur de cet hyperlien a relié le mot " verre » et la description du champ de fleurs parce qu'il les a considérés comme devant être reliés. Mais c'est au lecteur, sans doute d'abord déconcerté par sa découverte, d'élaborer des stratégies d'interprétation qui remédient au moins partiellement au manque de contiguité apparent. Dans ce cas précis, ces stratégies n’aboutiront peutêtre jamais à un résultat stabilisé : dans certains procédés rhétoriques du texte numérique hyperlié, des « lieux d’indétermination » [Iser, 1976 ; 1995] entre texte géniteur et texte relié continuent à résister, au moins partiellement, aux tentatives d'interprétation, et incitent le lecteur à investir encore et encore son imagination. Ce genre de renvois hypertextuels insolites sontils rares ? Pas tant que cela, comme je le montrerai plus loin.

Avant d'être activé, l'hyperlien fait rêver le lecteur à un tissu de potentialités. Après être activés, certains hyperliens peuvent continuer à alimenter ce rêve. Cette oscillation que l'on pourrait rapprocher du fonctionnement de la métaphore est sans doute moins rassurante que la confirmation ou l'explication d'annonces faites dans le texte géniteur (du genre : le verre est relié à l'image d'une bouteille de champagne). Au lieu de révéler des vérités, un hyperlien " métaphorique » augmenterait plutôt l'incertitude du lecteur sur le monde.

Pour accepter cette incertitude, le lecteur doit être préparé. Les procédés rhétoriques du texte numérique ne font pourtant pas encore partie de ceux que nous apprenons à reconnaître et à maîtriser à l'école. Alors que nous sommes sensibilisés aux procédés de l'argumentation dans le texte écrit et parlé, alors que nous savons que beaucoup de textes s'échangent dans notre vie quotidienne non pas dans le but d'informer, mais dans celui 
de persuader et de convaincre, l'hyperlien est souvent approché par le lecteur avec une attente de transparence informationnelle : de multiples études empiriques seront convoquées au chapitre II de ce livre pour donner du fondement à cette observation.

Si toute situation réelle est entourée d'un tissu de potentialités, et si l'hyperlien nous fait effectivement rêver à ce tissu, beaucoup de lecteurs semblent en effet espérer qu'il réduira leurs incertitudes dès qu'il aura été activé. C'est précisément pourquoi l'hyperlien peut être utilisé de façon si efficace pour induire le lecteur en erreur ! " Erst denken, dann clicken », « Réfléchir d'abord, cliquer ensuite » s'intitule une récente initiative allemande lancée sur Facebook, visant à alerter les lecteurs sur les incitations truquées au clic < http://www.facebook.com/fakepostings >. Non seulement celles-ci promettent plus qu'elles ne tiennent leurs promesses, mais déclenchent parfois des altercations indésirées ou des intrusions fallacieuses dans les données personnelles des utilisateurs.

\section{Promesses de I'hyperlien}

Si on réduit l'hyperlien à sa fonction de relier des " nœuds » d'information, on contribue à alimenter l'utopie d'un réseau de données dont il suffirait de comprendre la structure pour que son potentiel se déploie en toute transparence. On néglige alors le fait que la lecture d'un hypertexte se produit toujours en plusieurs phases, avant, pendant et après le geste d'activation effectué sur l'hyperlien.

Avant d'être activé, l'hyperlien adresse une promesse au lecteur. Les attentes du lecteur sont orientées par le mot hyperlié, par le texte entourant l'hyperlien, par les formes de la page-écran et par des imaginaires personnels et socialement partagés. C'est donc en fonction de tous ces éléments que le lecteur considérera, après activation, la promesse donnée par l'hyperlien comme plus ou moins tenue, mise en doute ou trahie. Encore faut-il que le lecteur procède à l'activation, et qu'il ne se contente pas d'une confiance aveugle dans la transparence du texte numérique. Encore faut-il que le lecteur accepte que l'hyperlien soit à la fois un outil d'information et de communication, et que cette dernière fonction donne lieu à des stratégies d'argumentation subtiles, comme à des duperies.

L'identification des différentes figures de cette relation de contiguïté établie par l'hyperlien entre deux textes, en fonction des attentes et imaginaires du lecteur, est l'un des enjeux fondamentaux de la rhétorique du texte numérique. Entre l'exemple d'un hyperlien répondant 
potentiellement aux attentes informationnelles du lecteur (le verre relié à une illustration de son contenu liquide) et un hyperlien défiant ces attentes (le verre relié à un champ de fleurs), une typologie commence à se mettre en place. Elle sera précisée et affinée au chapitre III.

\section{Le caractère impliquant du texte numérique}

L'hyperlien fait-il autre chose que relier deux textes ? Imaginons de déplacer l'hyperlien dans l'énoncé " je bois un verre » du verre vers le verbe. S'afficherait donc la phrase « je bois un verre ». Supposons maintenant que l'hyperlien relie de nouveau l'énoncé à la représentation du contenu buvable de ce verre. En cliquant sur "bois », n'avons-nous pas, du moins un peu, l'impression d'effectuer le geste évoqué ? Se dessinent ici les contours d'une rhétorique de l'immersion qui a souvent été négligée par la recherche académique.

Pendant longtemps, le texte numérique a en effet été considéré comme fondamentalement non immersif. Les premiers théoriciens de l'hypertexte [Landow, 1997 ; Bolter, 1991] ont forgé l'imaginaire d'un hyperlien comme outil de distanciation : l'hyperlien n'expose-t-il pas avant tout la marque de fabrique d'un texte numérique, alors que la lecture immersive nécessite une interface de lecture transparente ? L'hyperlien n'interrompt-il pas de toute évidence le flux de la lecture, alors que la plongée ininterrompue est une condition indispensable de toute immersion ? Ces observations laissent pourtant de côté l'implication corporelle du lecteur dans le texte numérique. Couplée à certains mots ou images, cette implication peut devenir hautement signifiante, dépassant de loin le rayonnement du geste mécanique lorsque le lecteur tourne les pages d'un livre.

Le texte n'est pas qu'un document. L'hyperlien ne fait pas que lier des textes. Les créateurs de publicités interactives en ligne ont depuis longtemps compris la nature impliquante de certains gestes de manipulation et l'explorent dans des créations où le lecteur plonge, boit, commande, rattrape, caresse des objets dans des mondes de textes et d'images immersifs.

L'hyperlien est-il donc par définition immersif, parce que manipulable ? Le geste de manipulation doit être pris en compte dans tout énoncé hypertextuel : il peut notamment induire certaines pratiques qui ne sont plus fondées sur un déchiffrement du texte, mais sur le pur plaisir du geste, éventuellement mis au profit d'enjeux industriels (comme dans la publicité en ligne, voir le chapitre III). 
L'énoncé manipulable « je bois un verre » perd cependant une part de son caractère immersif s'il est inséré non pas dans une hyperfiction ou une publicité, mais dans un reportage journalistique publié sur le site d'un magazine comme lexpress.fr : la lecture immersive, qui exige de s'adonner à la sensation de boire un mot en lisant et en cliquant, défie sans doute les attentes d'un lecteur à la recherche d'informations neutres et objectives. L'immersion paraît en effet difficilement compatible avec certains genres textuels. Pourtant, des expérimentations dans ce domaine existent, comme je le montrerai plus loin à partir de l'analyse du corpus journalistique (chapitre III, « Figures de la lecture du texte numérique dans les discours informatif et argumentatif » p.116).

Le caractère impliquant de certains " énoncés de gestes » rapproche le texte manipulable du texte animé : des entités non textuelles, le geste et/ou le mouvement, irradient potentiellement le texte à lire et orientent la pratique de celui-ci. Dans certains cas, ce phénomène est poussé à l'extrême : il se crée, dans le couplage entre le texte et le geste et/ou le mouvement, un " simulacre de référent » qui va bien au-delà de ce qui se passe dans l'exemple « je bois un verre ».

Dans un épisode de l'hyperfiction allemande Zeit für die Bombe ${ }^{3}$, le lecteur est invité à activer la phrase " appuie sur le petit interrupteur " (c'est moi qui traduis) pour déclencher le compte à rebours d'une bombe qui explosera plus loin dans l'histoire. En appuyant sur l'hyperlien tout en le lisant, le lecteur a sans doute au moins partiellement l'impression de manipuler lui-même l'interrupteur de la chose bombe : il est immergé dans l'histoire racontée en tant qu'acteur grâce à la forte ressemblance entre le geste physique effectué et le geste raconté.

Le texte relié lui apprend ensuite que la bombe a été activée par l'un des personnages. Dans l'énoncé « et la *bombe* faisait tic-tac », le mot « bombe » clignote $^{4}$ : autre effet posé sur le texte qui semble rapprocher celui-ci de son "référent d'expérience », la chose bombe. Il est important de prendre en compte le plaisir sensuel engendré par ces couplages entre texte, gestes et mouvements.

Pour cerner ce potentiel, la rhétorique propose des repères qui seront précisés dans les pages qui suivent. Avant de rentrer plus en détail dans les spécificités du texte numérique, il paraît nécessaire de revenir brièvement à l'histoire de la rhétorique et à ses enjeux contemporains.

3. Susanne Berkenheger, Zeit für die Bombe, [en ligne] : < http://www.wargla.de/zeit.htm >.

4. Les mots animés du texte numérique seront signalés par deux étoiles. 


\section{RHÉTORIQUE ET RÉCEPTION}

Depuis ses origines antiques, la rhétorique est considérée comme une science à la fois théorique et pratique. Oswald Ducrot et Jean-Marie Schaeffer la définissent comme un " art de la construction des discours " et comme une "théorie de ces discours » $[1995,166]$. Beaucoup d'auteurs [entre autres Bautier, 1994 ; Tétu, 2002] considèrent la rhétorique comme la première science de la communication en insistant sur son ancrage pragmatique : la rhétorique s'intéresse plus à la parole en acte qu'au système de la langue, et se penche sur les éléments de la pratique discursive : contenus, contextes, publics potentiels et réels. Discipline à vocation généraliste, la rhétorique étudie les discours d'historiens, de philosophes, de publicitaires, de journalistes, de juristes... " Analysant les moyens par lesquels les hommes communiquent publiquement » [Ducrot, Schaeffer, 1995, 167], s'occupant de la " forme du discours quand il devient public » [Bautier, 1994, 107], la rhétorique est plus souvent associée à la persuasion et au discours public qu'à la littérature.

La frontière entre la littérature et la rhétorique n'est pourtant pas étanche. Certes, la pensée classique a proposé une distinction entre la rhétorique spécialisée dans l'argumentation et la poétique « œuvrant dans le monde de l'imaginaire » [Klinkenberg, 1996, 339]. Aristote a cependant déjà montré qu'une même métaphore peut relever à la fois de la rhétorique et de la poétique. Alors que Chaïm Perelman, représentant de la "nouvelle rhétorique » qui a émergé ces dernières décennies, affirme qu' « une figure est argumentative si son emploi, entraînant un changement de perspective, paraît normal par rapport à la nouvelle situation ainsi suggérée » [2009, 61], et mobilise le terme problématique de " norme » pour distinguer la figure argumentative de la figure poétique, Oswald Ducrot et Jean-Marie Schaeffer avancent que la rhétorique, "base de tout discours ", est « a fortiori celle de toute littérature » [1995, 174]. C'est cette définition élargie de la rhétorique que je retiens ici.

La rhétorique se décline traditionnellement en cinq composantes qui circonscrivent ses champs d'action pratiques et théoriques [voir la reprise de ces composantes par Klinkenberg, 1996, 335-337]. L'inventio est centrée sur la recherche d'arguments. La dispositio ordonne ces arguments et applique en outre, selon le genre et le contexte, des « règles logiques » (du particulier vers le général, de la cause à l'effet...). L'elocutio désigne l'étape de la mise en forme des arguments, mobilisant par exemple des figures de style comme la métaphore ou l'allitération. L'actio concerne l'actualisation 
du discours dans un contexte de réception. La memoria enfin repose sur des techniques de mémorisation du discours.

Parmi ces cinq composantes, ce sont la disposition comme art de la composition et l'élocution comme art du style qui ont souvent retenu l'attention des théoriciens, créateurs et pédagogues. Ils me paraissent effectivement importants à prendre en compte aussi dans le cas du texte numérique. Comme le fait remarquer Chaïm Perelman [2009, 182], l'ordre de présentation des éléments par la disposition «modifie les conditions d'acceptation du discours ». Concernant l'hypertexte, j'ai déjà insisté sur le fait que le texte relié à un texte géniteur n'est généralement pas visible sur la page-écran avant d'être activé. L'ordre de lecture entre texte géniteur et texte relié compte alors pour beaucoup dans la réception si l'hyperlien est activé, notamment lorsque les deux textes reliés sont porteurs de points de vue divergents. Mais bien évidemment, le lecteur peut aussi omettre l'activation de l'hyperlien et passer à côté des explications, rectifications ou contre-arguments apportés par le texte relié.

Il risque d'en être ainsi dans l'exemple d'un article journalistique contenant une affirmation qui se trouve démentie dans le texte relié. Selon l'auteur d'un article du post.fr (texte numéro 12 du corpus, voir liste p. 237), les propos d'une jeune auto-entrepreneuse lors d'une émission de télé consacrée au gouvernement Sarkozy ont été très complaisants : " on apprenait par la suite qu'elle était fan de François Fillon ». Les lecteurs qui omettent d'activer l'hyperlien retiennent sans doute l'affirmation que la femme est fan, supposant que l'activation de l'hyperlien ne fait que confirmer cette idée. Or, le texte relié à cet hyperlien montre que la femme conteste cette affirmation.

Dans le texte animé, la disposition des éléments du discours joue de même un rôle clé dans la réception. Dans une publicité animée (voir chapitre III, " Figures de la lecture dans le discours persuasif », p. 217), une « offre spéciale » de réductions clignote d'abord à plusieurs reprises. Ensuite seulement s'affichent les " conditions particulières » et autres restrictions de l'offre. Cette disposition oriente l'attention du lecteur en priorité vers l'offre de réductions.

Cet exemple montre aussi que l'animation textuelle dans les bannières publicitaires relève souvent à la fois de la disposition qui crée des relations logiques (par le remplacement progressif du texte de l'offre par le texte des conditions) et de l'élocution qui met l'emphase sur les textes à lire (par le clignotement). 


\section{Éléments d'histoire}

Les deux exemples précités font apparaître un autre aspect important de la rhétorique : le rapport entre argumentation et vérité. Platon avait critiqué les sophistes et autres défenseurs de la rhétorique pour être plus préoccupés à flatter le public qu'à enseigner la vérité. L’amalgame entre rhétorique et tromperie a traversé les millénaires et rejaillit périodiquement. Il a participé au déclin de la discipline à l'époque moderne.

Pour Roland Barthes [1970], ce déclin s'explique en effet par la valeur progressivement attribuée à l'évidence. Oswald Ducrot et Jean-Marie Schaeffer [1995, 175] affirment de même qu'à l'aube de l'époque moderne, une rupture entre expression et argumentation a eu lieu parce que la philosophie rationaliste et l'empirisme voulaient dénoncer l'imprécision $\mathrm{du}$ « probable » et du « vraisemblable » dans l'argumentation. Pour Chaïm Perelman [2009, 20], l'ouvrage d'Omer Talon publié en 1572 a marqué une étape importante dans ce processus de déclin : la figure de rhétorique y a été définie comme une expression par laquelle l'allure du discours diffère de la droite et simple habitude. La rhétorique n'était plus qu'un art du langage " orné ». Elle est devenue, selon la célèbre formule de Gérard Genette [1970], une " rhétorique restreinte », centrée sur l'élocution.

Pour Jean-Marie Klinkenberg [1996, 339], ce déclin de la rhétorique va de pair avec l'évolution de la société. C'est dans des périodes de mise en péril de la démocratie que la rhétorique se trouve, selon l'auteur, réduite à l'étude des ornements. Jean-Marie Klinkenberg met ainsi l'accent sur la fonction sociale d'une discipline qui étudie les usages stratégiques $\mathrm{du}$ discours en évaluant de façon critique les choix faits par les auteurs, concepteurs et commanditaires.

La « nouvelle rhétorique » qui a émergé au xx siècle répond, selon Roger Bautier [1994], d'une part, « à l'exigence sociale d'une maîtrise de la parole efficace adaptée aux médias modernes », et d'autre part, à l'« intérêt scientifique pour le fonctionnement des argumentations » (281). De nouveau en phase avec l'évolution des sciences sociales, cette rhétorique se penche en particulier sur « la fonction de délibération en situation d’incertitude » (108). 


\section{Culture de l'évidence - culture de l'argumentation}

Bruno Latour [1986] figure parmi les auteurs qui ont contribué à la réhabilitation de la rhétorique lors de la seconde moitié du $\mathrm{xx}^{\mathrm{e}}$ siècle. L'auteur critique l'idée selon laquelle la rhétorique, définie comme un " art de la persuasion ", s'éloigne de l'idéal du discours scientifique : au contraire, le rôle de la rhétorique est essentiel dans toutes les sciences, sciences « dures » incluses.

Afin de mieux comprendre cette réhabilitation engagée par Bruno Latour, il me paraît utile de convoquer la distinction proposée par Philippe Breton et Serge Proulx [1989] entre une « culture de l'argumentation » reposant sur la mise en discussion et le croisement de points de vue, et une « culture de l'évidence » mettant en avant la nécessité de l'évidence rationnelle ou expérimentale.

En effet, comme je l'ai déjà indiqué, la rhétorique s'est souvent trouvée mise en cause parce qu'elle privilégierait le vraisemblable et le plausible sur le vrai et la certitude, et qu'elle chercherait à provoquer l'adhésion du public par la séduction. Au $\mathrm{xx}^{\mathrm{e}}$ siècle, la théorie classique de la connaissance prônant l'évidence s'est pourtant trouvée progressivement concurrencée par une théorie néo-kantienne montrant comment toute connaissance est aussi « conditionnée par les a priori du sujet connaissant » [Bautier, 1994, 83]. C'est dans ce sens que Bruno Latour [1986] peut plus récemment affirmer que même le fait scientifique est construit. "Tous ceux qui croient pouvoir dégager la vérité indépendamment de l'opinion n'ont que mépris pour la rhétorique qui porte sur les opinions ", affirme Chaïm Perelman [2009, 23-24]. « Mais tous ceux qui croient à l'existence de choix raisonnés, précédés par une délibération ou une discussion, où les différentes solutions sont confrontées les unes aux autres, ne pourront se passer, s'ils désirent acquérir une claire conscience des méthodes intellectuelles utilisées, d'une théorie de l'argumentation telle que la présente la nouvelle rhétorique ».

Dans la courte histoire de l'hyperlien, cette oscillation entre une culture de l'argumentation et une culture de l'évidence se retrouve. D'une part, un postulat de "transparence informationnelle » a forgé un certain nombre d'imaginaires de l'hyperlien, notamment en informatique ; d'autre part, l'hyperlien a été investi, notamment par la littérature numérique, du rôle de révélateur d'incertitudes incitant à la délibération. Je mènerai au chapitre II une étude plus précise sur ces imaginaires, qui ont parfois freiné le dialogue entre les disciplines intéressées par le texte numérique. 
Je montrerai au chapitre III comment les figures du texte numérique anticipent sur ces imaginaires.

\section{Rhétorique et pouvoir}

Le plaidoyer de Latour et Perelman pour une " nouvelle rhétorique » révèle à quel point tout processus d'argumentation et de persuasion est non seulement conditionné par les procédés rhétoriques de la disposition et de l'élocution, mais aussi par des incidences politiques et sociales. Pierre Oléron [1983] insiste sur le fait que l'argumentation relève à la fois de l'ordre du raisonnement (établissement de liaisons entre des propositions) et de l'influence (exercice de sollicitations pour faire accepter une proposition). Les analyses rhétoriques ont donc souvent été justifiées par le fait que le sujet a besoin de connaître les stratégies du discours pour devenir autonome face à ces sollicitations. Parmi une multitude de manuels dédiés au décodage du discours persuasif, l'on peut citer celui de Howard Kahane [1976]. Derrière ce genre d'approche émerge la question centrale de la place du lecteur, et de ses possibilités de résistance face aux procédés et stratégies mobilisés par la rhétorique.

Les préoccupations de la rhétorique rejoignent ainsi l'évolution des grands courants de la sociologie. Alors que jusqu'en 1940 primait l'idée que le discours, et notamment le discours propagé par les médias, pouvait influencer le public de façon directe et inexorable (modèle « hypodermique »), des approches plus récentes ont mis en question cette représentation de la communication. S'est imposé dans les sciences sociales et les études littéraires, la linguistique et la rhétorique, le modèle d'un lecteur « actif».

\section{La place du récepteur dans le discours}

La place du récepteur, sa liberté et ses contraintes face au discours se trouvent désormais au centre des débats théoriques sur la rhétorique. Se pose, autrement dit, la question de savoir si les figures de rhétorique ont un effet immédiat et obligatoire sur le récepteur, ou s'ils ne constituent que des potentialités qui s'actualisent de la façon la plus diverse dans une situation de réception précise. 
Le philosophe grec Zénon [voir Quintilien, vol. I, livre II, chap. XX, § 7] a comparé la rhétorique à une " main ouverte ", que le public est libre de saisir ou non. Critiquant les typologies de figures exemplifiées dans grand nombre de manuels par des citations courtes, Chaïm Perelman [2009] affirme qu' " en examinant les figures hors de leur contexte, comme des fleurs desséchées dans un herbier, on perd de vue le rôle dynamique des figures : elles deviennent toutes des figures de style » (14). Il plaide donc pour une prise en compte d'un contexte plus large qui inclut le récepteur (28) : « il faut qu'un discours soit écouté, qu'un livre soit lu, car, sans cela, leur action serait nulle».

Même si Chaïm Perelman considère donc le récepteur comme un élément déterminant dans la "valeur » de l'argumentation, il ne va pas jusqu'à mobiliser des approches empiriques pour circonscrire cette actualisation du discours. Comme Wolfgang Iser et Hans Robert Jauss, fondateurs d'une théorie de la réception que je convoque amplement dans ce livre, Perelman [2009] recourt donc au modèle d'un " lecteur implicite » et définit les récepteurs comme « l'ensemble de ceux sur lesquels l'orateur veut influencer par son argumentaire » (32).

\section{Potentialités du message, formes incluses}

Michel de Certeau [1990], souvent cité pour argumenter en faveur d'un recentrage sur le lecteur, a recensé un certain nombre de possibilités dont celui-ci dispose pour faire face aux procédés de "manipulation » de la rhétorique. Roger Bautier [1994] fait pourtant remarquer qu'il y a parfois "surestimation du pluralisme des messages comme des capacités d'intervention de ceux auxquels ils s'adressent » (10). Toute analyse rhétorique, qu'elle porte sur un texte papier, une émission télé, une image ou un texte numérique, comporte en effet soit le risque d'une surestimation de l'effet des figures mobilisées par le texte, soit celui d'une surestimation des pouvoirs du récepteur. Face à ces risques, Roger Bautier [1994] défend une démarche "marquée par la prudence et la référence aux aspects sociologiques ou historiques de la communication ", mais qui " ne peut se passer d'une réflexion d'ordre sémiologique et linguistique " pour évaluer les «potentialités offertes par les différents moyens de communication » (53). Je rejoins tout à fait Roger Bautier pour affirmer qu'il s'agit donc de sonder les " marges de manœuvre " dont dispose le récepteur « sans s'illusionner sur sa liberté ni la sous-estimer » (146). Si des études 
empiriques par questionnaire ou entretien peuvent fournir des renseignements précieux pour examiner le rôle des attentes et imaginaires dans le processus de réception, la rhétorique propose des méthodologies pour l'examen des potentialités du texte dans son contexte de production et de réception matérielles.

Alors que les approches rhétoriques du texte papier remplissent des bibliothèques entières, l'étude sémiotique et rhétorique du texte numérique et de ses spécificités sur la page-écran reste encore un champ de recherche peu défriché. Ce livre identifie, pour la première fois de façon exhaustive, les stratégies et procédés rhétoriques du texte manipulable et animé. Les potentiels d'action du texte numérique seront circonscrits tout en prenant en compte la complexité de l'activité de réception entre perception, interprétation et plaisir.

La question du plaisir fait surgir une composante traditionnelle de la rhétorique qui n'a pas toujours été suffisamment conceptualisée : la paralinguistique, donc le geste, le son, le mouvement, bref l'implication du corps avec tous ses sens. Même si la rhétorique a été dès Quintilien associée à l'expression corporelle, les éléments comme le geste ou l'oralité ont souvent été écartés par la suite. L'implication du corps du lecteur dans le texte numérique, dont la sensualité des gestes d'appui et de relâchement présents dans tout hyperlien, a notamment été négligée par les premiers théoriciens de l'hypertexte [Landow, 1997 ; Bolter, 1991]. Le mouvement dans le texte animé s'est trouvé fréquemment relégué du côté du ludique. Afin de réhabiliter la complexité de ce caractère " impliquant » du texte numérique, j'aurai notamment recours à des outils d'analyse sémiotiques. L'intégration de la sémiotique dans la rhétorique du texte numérique fait surgir la question complexe des rapports entre rhétorique et sémiotique que j'effleure seulement ici, car elle deviendra palpable lorsque ma méthodologie sera mise à l'épreuve des exemples du corpus.

\section{Rhétorique et sémiotique}

Comme le rappelle Sémir Badir [2011], l'étude de la langue et des autres "systèmes de signes » est, depuis la « linguistique générale » de Ferdinand de Saussure, souvent attribuée à la linguistique et à la sémiotique ; la rhétorique s'occupe de la " parole en acte ». La rhétorique apparaît ainsi comme la partie créative et ouverte du système sémiotique [voir 
Klinkenberg, 1996] qui se penche sur la production de nouvelles relations entre « unités » de sens et la production de nouvelles unités.

Ferdinand de Saussure [2002, 26] a cependant reconnu que l'un des problèmes les plus difficiles en linguistique concerne justement la définition des unités et de leur différence, car « la nature de l'unité linguistique est d'être relationnelle ». Se pose donc toujours la question de l'existence préalable des unités sémiotiques avant leur mise en relation dans des figures de rhétorique. Pour souligner à quel point le système sémiotique est en réalité dynamique, Saussure remplace dans certains de ses textes le terme de « différence » par celui de « valeur » : «C'est le dynamisme et la variation qui spécifient le système de valeurs linguistique », commente Sémir Badir [2011].

En me penchant dans ce livre sur les « unités sémiotiques de la manipulation » et les " unités sémiotiques temporelles » impliquées dans le texte numérique hyperlié et animé, je ferai certes comme si ces unités préexistaient à leur relation. J'insiste cependant dès maintenant sur le fait que je crée, à ce moment, une situation d'observation " en laboratoire », à laquelle je remédierai ensuite en reconstruisant le plus minutieusement possible le contexte de réception. J'adopterai alors une méthodologie que j'appelle " sémio-rhétorique », tout en étant consciente du champ de tensions qui traverse ce terme.

\section{UNE THÉORIE DE LA RÉCEPTION DU TEXTE NUMÉRIQUE}

Suivant les approches de la « nouvelle rhétorique » que je viens d'esquisser, tout texte préfigure donc son lecteur et ses lectures par des procédés spécifiques, qu'il argumente une prise de position, qu'il relate méticuleusement des faits ou qu'il invite le lecteur à entrevoir des "vérités métaphoriques » [Nietzsche, 1993, 181-182]. Tout texte reconfigure par ailleurs le réel, qui ne peut être décrit de façon transparente : ainsi que le réaffirme Yves Citton [2010, 149] suite à Bruno Latour, même les faits scientifiques se fabriquent, car ils sont toujours le résultat d'une interprétation. L'objectif de la rhétorique a toujours été de maîtriser et de révéler les outils déployés lors de cette interprétation du réel.

La réception du texte numérique est définie comme une rencontre, au moins partielle, entre un texte avec ses procédés rhétoriques et ses formes sur la page-écran, et l'horizon d'attente d'un lecteur doté de ses capacités, centres d'intérêt et référents culturels, membre d'une société avec 
laquelle il partage une ou plusieurs langues, valeurs, pratiques et imaginaires. Certains chercheurs [par exemple Courbet, 2004, 20] affirment que l'étude sémiotique et rhétorique des discours médiatiques devrait avoir comme objectif de construire des hypothèses ; il incomberait ensuite aux spécialistes de la lecture de les tester empiriquement. Je propose dans ce travail une autre démarche : l'approche rhétorique est informée en amont par les résultats d'études empiriques, inspirées en outre de méthodologies sociologiques; aucune « figure de rhétorique » du texte numérique ne sera identifiée sans prise en compte de l'horizon d'attente potentiel du lecteur.

\section{Théories de la réception du texte}

Mon approche de la réception du texte numérique s'inscrit dans les filiations pragmatiques de la rhétorique et emprunte également des concepts clés à la « théorie de la réception » développée par l’École de Constance en Allemagne [notamment par Wolfgang Iser et Hans Robert Jauss], et plus récemment par Stanley Fish [1980] aux États-Unis et Bertrand Gervais [1998 ; 2007] au Canada.

Hans Robert Jauss [1978 pour la traduction française] insiste en particulier sur la nécessité d'une approche philologique lorsqu'il s'agit de reconstruire l'« horizon d'attente » des lecteurs d'un texte : le texte est considéré comme le résultat d'une convergence entre sa "structure donnée » et sa " réception » par le lecteur, réception qui est à son tour culturellement et historiquement déterminée. Le texte est donc perçu comme une « structure dynamique » qui ne peut être saisie que dans « ses concrétisations historiques successives » [2010, 269]. Lorsqu'il est possible de reconstruire l'horizon d'attente des premiers lecteurs ou des lecteurs à d'autres moments précis de la vie sociale d'un texte, et que le potentiel d'action du texte est donc croisé avec des témoignages de lecteurs, cette méthodologie révèle toute son efficacité (voir chapitre III, « Figures de la lecture et formesmodèles de la page-écran dans le discours narratif », p. 183).

Dans une approche plus synchronique, Wolfgang Iser propose dans L'acte de lecture [1995 pour la traduction française] une théorisation générale de la réception, et met l'accent sur le repérage précis des " répertoires » et «stratégies » qui guident la lecture : méthodologie qui s'apparente aux approches " sémio-pragmatiques » pratiquées dans les sciences de l'information et de la communication françaises. Lorsque Jean-Jacques Boutaud et Eliseo Veron proposent ainsi dans leur ouvrage Sémiotique 
ouverte [2007, 146] d'analyser la lecture comme un champ de tensions entre la " potentialisation » opérée par le texte et l'" actualisation » forcément partielle de ce potentiel par le lecteur, ils mobilisent des concepts clés avancés également par Wolfgang Iser. L'auteur considère en effet le texte comme un " potentiel d'action » et l'acte de lecture comme une « actualisation » créative.

Les objets textuels étant pertinemment transformés par la pratique des lecteurs, le processus de lecture ne pourra jamais être complètement prescrit et prédit. Certes, l'auteur d'un texte élabore de façon consciente ou inconsciente des hypothèses concernant son lecteur (voir la théorie $\mathrm{du}$ « lecteur modèle » [Eco, 1985], du « lecteur implicite » [Iser, 1976 ; 1995] et de l'« auditoire universel » [Perelman, 2009]). Certes, le texte active, comme le formulent Jean-Jacques Boutaud et Eliseo Veron [2007, 172], certaines trajectoires au détriment d'autres trajectoires, et l'auteur propose ainsi une place au lecteur. Certes, le lecteur nourrit des attentes concernant les caractéristiques du texte. Il paraît évident qu'une compréhension peut seulement avoir lieu si les propositions du texte et les attentes du lecteur se rencontrent au moins partiellement. Néanmoins, il ne faut pas oublier ce qui caractérise fondamentalement cette rencontre entre texte et lecteur, sur papier comme sur support numérique : la discontinuité de la communication par le texte, qui fait que celui-ci pourra être actualisé par différents lecteurs, à différentes époques. Toutes les approches de la rhétorique tournées vers la prise en compte du lecteur ont insisté sur le fait que l'altération du texte par le lecteur est inépuisable. Le texte échappera toujours à son auteur, comme à son récepteur.

\section{Anticipations de lectures par le texte}

Même si l'horizon textuel et l'horizon d'attente du lecteur se trouvent inextricablement liés, il paraît important de se demander « dans quelle mesure les processus d'élaboration déclenchés par le texte sont anticipés par celui-ci » [Iser, 1995, 9]. Même si la réception doit, selon Iser, être considérée comme un processus créatif, elle n'est en effet pas un "rêve éveillé ». Le lecteur se " branche » [Iser, 1995, 71] sur un texte qui l'engage vers un point de vue. Le lecteur n'est donc pas entièrement libre de choisir ce point de vue, qui est préfiguré en outre par la perspective dans laquelle un texte est présenté, par sa disposition et son élocution. Le texte donne, de manière anticipée, son mode de réception et libère 
un « potentiel d'action » [Iser, 1995, 13] : en rencontrant, au moins partiellement, les attentes et savoirs de ses lecteurs, le processus de lecture peut susciter la satisfaction ou alors la surprise, la déception, voire la déconcertation.

\section{Répertoires et stratégies}

Wolfgang Iser utilise le terme " répertoire » pour désigner toutes ces allusions aux événements contemporains et historiques, les références explicites ou implicites à d'autres textes (renvois extra-textuels), les éléments déjà évoqués dans le texte que le lecteur est en train de lire (renvois intra-textuels) et les conventions sociales plus ou moins connues qui sont inhérentes à un texte. Par ses répertoires, le texte s'inscrit ainsi dans un monde social qui doit être au moins partiellement partagé par le lecteur. Jean-Jacques Boutaud et Eliseo Veron [2007, 33] préfèrent le terme de " cadre d'expérience » pour désigner ce que Algirdas Julien Greimas et Joseph Courtés [1986, 119] définissent comme "savoir socioculturel commun garantissant une interprétation suffisamment isotope du contexte extralinguistique à l'intérieur duquel la communication prend place », et qui constitue l'horizon d'attente. Y figurent aussi les imaginaires plus ou moins partagés. Certains « interprétants globaux » [Boutaud, Veron 2007, 19] ont le statut de "noyaux d'appartenance » auxquels tout lecteur dans une société a, à un moment donné, de fortes chances de s'identifier.

Avec Hans Robert Jauss et Jean Starobinski [2010, 14], je considère la lecture comme une " perception guidée », " un processus correspondant à des intentions et guidé par des signaux que l'on peut découvrir et décrire ». Le degré de détermination du "répertoire » est l'un des éléments qui préfigurent la connivence possible entre texte et lecteur. Wolfgang Iser cite comme exemple la littérature didactique, qui reprend dans son répertoire le système de valeurs prévalant potentiellement chez ses lecteurs dans le but de démontrer sa validité [1995, 152].

Les «stratégies » du texte relient les éléments du répertoire et ébauchent les conditions de perception du texte, ses « orientations opérationnelles » [Iser, 1976 ; 1995]. Ces stratégies correspondent, par exemple, aux connexions temporelles et logiques effectuées par la dispositio rhétorique : l'ordre des événements racontés peut ainsi créer plus ou moins de suspense dans un récit. L'exhaustivité des détails sur ces événements engendre plus ou moins de cohérence à l'intérieur du texte. 
Un texte numérique contient, comme tout texte, des « répertoires » et des « stratégies ». Il fait allusion à des normes sociales, des événements historiques ou des faits d'actualité, des personnalités connues, des termes renvoyant à un imaginaire (la « société d’information », la " crise de la dette »...), des croyances et valeurs partagées (le patrimoine, la famille, la protection des mineurs...), des intertextes « externes » (dictons, œuvres célèbres, émissions de télé à forte audience...) et " internes » (allusions à des faits, relations, événements exposés auparavant dans le texte...), bref une multitude d'éléments qui font potentiellement partie d'un savoir commun entre le texte et le lecteur, présentés selon un certain ordre et créant les orientations opérationnelles du texte.

Certaines de ces composantes font en outre appel à des genres textuels : l'interview, le reportage, l'hyperfiction, le billet de blog personnel ou l'article d'encyclopédie, qui ont parfois une longue histoire dans l'univers de la publication papier - mémoire sociale qui préfigure également les conditions de réception.

\section{Annonces et reprises}

Tout texte contient des annonces de la suite qui suscitent des attentes chez le lecteur. Sur un dispositif de lecture papier, cette suite est présentée sur des pages dont l'existence et le volume peuvent être évalués facilement. Dans le cas d'un texte numérique avec des hyperliens, le texte géniteur propose d'abord une suite immédiatement visible, qui est présentée dans la continuité spatiale des mots hyperliés ${ }^{5}$. Le texte relié, activable par hyperlien, propose une deuxième suite du texte géniteur qui n'est pas immédiatement visible. En dehors du fait que le lecteur ne sait pas toujours laquelle des deux suites il est censé explorer en premier, l'existence, le volume et le contenu de ce texte relié peuvent seulement être présumés, à partir d'éventuelles annonces dans le texte géniteur. Les " annonces » sont donc une composante importante de la rhétorique du texte numérique, car elles peuvent aider à prévenir la désorientation du lecteur.

L'emplacement de l'hyperlien sur des mots précis joue également un rôle stratégique dans la rhétorique du texte numérique : celui-ci peut être posé sur un nom propre, un verbe, un chiffre, voire sur un signe de ponctuation, une phrase entière...

5. Un hyperlien est en effet rarement posé sur le dernier mot d'un texte ; en général, celui-ci continue après le mot ou la phrase hyperliés. 
Le renvoi au texte relié mobilise par ailleurs un certain nombre de représentations sociales : il peut constituer la trace d'une complicité ou d'une opposition, d'une filiation ou d'une dénonciation de l'autre texte, ou alors l'indice d'une obligation dictée par un tiers (par exemple dans le cas des hyperliens promotionnels). Comme le formule Astrid Ensslin [2007, 15], un hyperlien implique toujours l'existence de l'« autre ». Il rappelle, par certains côtés, les stratégies rhétoriques de la citation.

Antoine Compagnon [1979, 40] souligne que la citation constitue un mode de renvoi plus ambigu qu'il ne paraît, et il convoque les figures de Narcisse et Pilate pour circonscrire cette ambiguïté. Certes, la citation permet à l'auteur de se " miroiter » dans le texte écrit par quelqu'un d'autre, à la manière de Narcisse. En même temps, l'auteur désigne son emprunt du doigt et, comme Pilate, se disculpe. Au cas où le lecteur lui reprocherait sa prise de position, il peut toujours pointer les guillemets qui entourent l'emprunt. Je montrerai plus loin comment certains journalistes mettent à profit cette situation ambiguë entre adoption et distanciation en reliant deux textes porteurs de points de vue fortement divergents, sans prendre explicitement position en faveur ou à l'encontre de l'un d'entre eux. Le recours à l'autre texte par hyperlien implique dans ce cas à la fois l'opportunité d'une identification, et la possibilité d'une prise de distance. Il laisse le lecteur seul avec ses incertitudes.

Alors que certains journalistes préfèrent préserver cette ambiguïté du renvoi à l'autre (texte), d'aucuns adoptent le texte relié jusqu'à inclure des parties dans le texte géniteur. L'emprunt peut être plus ou moins explicite. Parfois il est affecté de guillemets, parfois la présence de l'hyperlien reste son seul marqueur.

\section{Degrés de contiguïté}

Les répertoires et stratégies du texte géniteur, ainsi que certaines annonces et reprises orientent les attentes du lecteur concernant le texte relié, qui propose, à son tour, un potentiel d'action : il fait appel à des représentations sociales, des événements historiques, des croyances et valeurs partagées. Comme dans tout texte, le lecteur attend une certaine contiguïté de l'enchaînement entre le texte géniteur et le texte relié.

La mise en relation de deux textes par hyperlien résulte en effet, comme je l'ai déjà indiqué à plusieurs reprises, d'un acte d'interprétation par l'Auteur : celui-ci pose un lien entre deux textes parce qu'il les considère comme devant être mis en relation. La contiguïté plus ou moins forte 
entre texte géniteur et texte relié en fonction des répertoires, stratégies, annonces et reprises d'une part, et en fonction de l'horizon d'attente du lecteur d'autre part, permet la constitution d'une situation de réception plus ou moins satisfaisante, surprenante ou déconcertante. Les niveaux de contiguïté, allant d'une confirmation complète des attentes potentielles du lecteur jusqu'à une forte mise au défi de celles-ci, sont un élément fondateur de la rhétorique du texte numérique.

\section{MATÉRIALITÉS DU TEXTE, DE LA PAGE-ÉCRAN ET DU DISPOSITIF}

Même si les niveaux de contiguïté entre des textes reliés par un hyperlien participent certainement de façon importante à leur potentiel d'action, les textes manipulables et animés sur support numérique ne sont pas que des textes à lire, mais aussi des textes à regarder et à manipuler. Ces dimensions matérielles du texte font partie du champ plus large des « matérialités de la communication » [Gumbrecht, 2010, 26]. Les " matérialités de la communication », écrit Gumbrecht, "sont tous les phénomènes et les conditions qui contribuent à la production d'une signification sans constituer elles-mêmes cette signification » (26). Par le terme " signification ", Gumbrecht désigne ici l'énonciation discursive du texte, alors que les phénomènes et conditions qui contribuent à sa production s'apparentent à ce qu’Emmanuël Souchier [1998] appelle l'« énonciation éditoriale ».

Partant de la critique du logocentrisme par Jacques Derrida [1967] qui a montré comment la domination de la signification et de l'esprit s'est affirmée dans la culture occidentale à partir du délaissement de la matérialité des supports d'écriture, Gumbrecht formule la question qui le préoccupe depuis les années 1980 : comment différents médias de communication affectent-ils la signification dont ils sont porteurs? « Nous avions cessé de croire qu'un complexe signifiant était indépendant de sa matérialité », écrit Gumbrecht, tout en se demandant " comment aborder cette interface entre signification et matérialité » [2010, 31]. Dans les approches plus récentes, ces matérialités de la communication sont considérées non plus seulement comme des "supports », mais comme des coproducteurs à part entière de la signification.

Marshall McLuhan avait formulé en 1964 une réponse radicale et provocatrice à la question posée par Gumbrecht : " Le médium est le message ». L'impact des médias sur l'humain est considéré par McLuhan 
comme avant tout structurel : il se situe en deçà des " contenus » que ces médias transportent, et au-delà des " usages » que l'humain en fait. L'analyse des contenus notamment ne peut, selon l'auteur, que manquer cette relation entre le technologique et le sensoriel : le message n'est plus le contenu échangé via le médium, mais sa forme. Si l'on adopte cette idée, cela équivaut en dernière conséquence, comme le souligne Samuel Archibald [2009, 88], à penser par exemple "l'imprimé sans penser le texte ni la lecture » : posture exclusive qui n'est évidemment pas tenable.

Les récentes théories de la réception visent en effet plutôt à étudier l'interaction entre le texte et ses matérialités en prenant en compte la relation avec le lecteur. Certaines approches développées ces dernières années fournissent des méthodologies clés pour circonscrire avec précision cette interaction. J'insiste avant tout sur l'importance de la pensée de l'« énonciation éditoriale » [Souchier, 1998 ; Jeanneret, Souchier, 2005] et de la «trivialité » [Jeanneret, 2008], qui étudient le texte comme " matière », mais aussi comme " mémoire des formes » constamment actualisée par les pratiques. Cette pensée des matérialités ou des « formesmodèles » de la communication, qui joue un rôle périphérique dans les théories de la lecture développées par Wolfgang Iser et Hans Robert Jauss, est théorisée par Emmanuël Souchier et Yves Jeanneret dans toute son ampleur nécessaire.

Longtemps négligée dans le domaine des recherches sur la lecture papier qui la souhaitait simplement la plus transparente possible, l'« énonciation éditoriale » a toujours joué un rôle important dans la coconstruction du sens par le lecteur. Cette " énonciation éditoriale » est définie comme " la trace objective d'une énonciation qui ne relève pas uniquement du linguistique, mais également de la pratique des métiers et, plus généralement, de la pratique sociale » [Davallon, Després-Lonnet, Le Marec et al., 2003, 29]. Comme le formule Yves Jeanneret [2008, 66], il faut donc prendre en compte la "représentation et anticipation des pratiques » inscrites dans l'objet lui-même, qui « définit les possibles d'une appropriation ».

Lorsque le lecteur lit un mot sur un support numérique, il perçoit en même temps son affichage sur un écran rétro-éclairé, sa forme graphique, son animation et sa manipulabilité, sa position dans le texte et sur la page-écran (ce mot « page » qui mobilise en soi tout un héritage culturel, voir Pignier, Drouillat, 2008, 36), son insertion dans des « cadres » de lecture qui imitent plus ou moins les supports de lecture antérieurs (par exemple, la page de journal ou de livre), ou surprennent au contraire par 
des formats inédits, bref toutes ces marques de l'« élaboration plurielle » [Souchier, 1998, 145] de l'objet textuel qui échappent en partie à l'auteur : notamment sur les supports numériques où la vitesse de calcul, le choix du navigateur, la présence ou l'absence de plug-ins et la résolution de l'écran peuvent profondément altérer l'actualisation du texte.

\section{Approches des « formes-modèles » du texte numérique}

Les textes manipulables et animés sur support numérique ne sont donc pas que des textes à lire. Ils combinent, sur le même support actif, plusieurs systèmes de signes. Du texte à lire d'une part, et d'autre part des «formes-modèles », pour utiliser le terme proposé par Yves Jeanneret et Emmanuël Souchier [2005] : la couleur, la forme et la taille des lettres, et plus spécifiquement le mouvement dans le cas du texte animé, et le geste dans le cas du texte manipulable. La nature différente des signes impliqués influe potentiellement sur l'acte de réception en sollicitant le corps du lecteur de façon autrement sensible que le texte à lire.

Éric Landowski [2004] inscrit le sensible comme élément important dans tout acte de communication, l'identifiant dans nos rapports à l'autre en tant que corps-sujet, dans la temporalité comme dans les objets du « goût ». Jean-Jacques Boutaud [2007] précise que le rôle du sensible dans la communication a cependant encore gagné en importance dans un monde où « le sens de l'expérience est guidé par la recherche de sensations, par l'immersion dans des contextes enveloppants, polysensoriels ». Le « régime dominant » actuel est, selon l'auteur, « celui de l'esthésie et des émotions sensorielles favorisées par synesthésie, par coopération des sens ».

Ces observations me paraissent primordiales pour cerner le caractère plurisémiotique du texte numérique. "Savons-nous prendre en compte la part sensible du sens ? », se demande Jean-Jacques Boutaud [2007]. J’ai développé ces dernières années une méthodologie permettant de circonscrire les conditions d'émergence de ces « synesthésies » dans le texte numérique. Alors que le couplage entre texte et mouvements ou entre texte et gestes de manipulation relève de la rhétorique, mobilisant plus particulièrement les composantes de la disposition et de l'élocution, je recours à des outils sémiotiques pour circonscrire le potentiel d'action des mouvements et des gestes de manipulation impliqués dans ces couplages.

Je propose en outre de théoriser la part sensible du sens dans le texte numérique à travers la notion d'« iconicité ». Jean-Marie Klinkenberg 
[1996] définit l'icône comme un signe " motivé par ressemblance ", qui peut transiter par d'autres canaux que la vue ; son signifiant est un ensemble modélisé de stimuli (supports matériels du signe) qui correspond à un " type stable, ensemble modélisé que l'on peut atteindre grâce au stimulus. Ce type est identifié grâce à des traits de ce signifiant, et peut être associé à un référent reconnu » (384-385).

Bien qu'un mot ne soit pas une icône [voir Michel Foucault, 1973], beaucoup de débats ont été menés sur une possible iconicité du texte alphabétique, sur les caractéristiques qui pourraient le rapprocher d'un « référent d'expérience ». Victor Hugo [1987, 684] note ainsi « combien l’Y est une lettre pittoresque qui a des significations sans nombre » : « L'arbre est un Y. L'embranchement de deux routes est un Y. Le confluent de deux rivières est un Y. Une tête d'âne ou de bœuf est un Y. Un verre sur son pied est un Y. Un suppliant qui lève les bras au ciel est un Y ». La police de caractère et la couleur d'un texte peuvent également être perçues sur le mode iconique.

Comme la couleur et la forme des lettres, certains mouvements et enchaînements de gestes semblent marqués par leur caractère iconique, qui fait autrement appel aux sens du lecteur que le texte lisible. Ce potentiel d'action que je proposerai plus loin d'appeler « référentiel » (à cause du renvoi vers un référent d'expérience), s'actualise aussi en couplage rhétorique avec le mot.

\section{Unités sémiotiques de la manipulation}

La manipulabilité de l'hyperlien a parfois été interprétée comme une « interactivité » qui donne au lecteur la possibilité de modifier le texte ; cette modification a été considérée comme équivalente à un acte d'écriture. Le néologisme du "wreader", combinaison entre auteur et lecteur [entre autres Landow, 2006], était né. Je pense que cette conception de la manipulabilité à la fois surestime et sous-estime le rôle des gestes mobilisés dans l'activation d'un hyperlien. Le terme " signe passeur » a été forgé par Yves Jeanneret et Emmanuël Souchier [1998], entre autres pour " prendre au sérieux ce que signifie une nouvelle forme de lecture gestualisée » [Jeanneret, Davallon, 2004, 50].

Peu de chercheurs se sont cependant penchés sur le rôle précis du geste dans son interaction avec le texte numérique - notamment dans le cas de l'hyperlien, cela semblait aller de soi. Astrid Ensslin [2007, 15] affirme, par exemple, que l'hyperlien a une fonction tripartite d'un point 
de vue rhétorique parce qu'il est composé à la fois d'un signe textuel, d'un indice sous forme de pointeur, et d'un «tremplin » vers un autre texte ; elle omet d'évoquer les gestes impliqués. Comme le formulent Nicole Pignier et Benoît Drouillat [2008, 204], il peut se dégager de l'hyperlien une "impression de relation neutre ». Il paraît investi avant tout de la fonction d'« intermédiation utilitaire entre les usagers et l'information ».

Nicole Pignier et Benoît Drouillat figurent néanmoins parmi les chercheurs qui sont allés le plus loin dans la mise en avant de l'importance du geste de manipulation dans la création numérique. Analysant le rôle $\mathrm{du}$ geste dans des sites commerciaux et dans l'art, ils font remarquer que pour des artistes comme Anne-Sarah Le Meur, le clic s'accompagne d'une impression de pouvoir et de maîtrise. Cette impression s'opposerait à celle suscitée par le roll-over, d'une intensité douce et d'une étendue plus large dans le temps, qui peut donc « être perçue par l'usager comme l'expression de la caresse ou encore de l'effleurement » [2008, 188, 189]. Les auteurs parlent également de " signes iconiques » lorsque s'établit une « relation d'équivalence » entre l'action de l'usager, son geste et un contenu interactif sur la page-écran (par exemple, une image) ; ils ne se penchent cependant pas sur la dimension iconique de l'hyperlien en tant que texte à lire.

Serge Bouchardon [entre autres 2002, 2009, 2011] a insisté à maintes reprises sur le rôle du " geste de manipulation » dans la réception d'une création numérique. Il a envisagé l'établissement d'un catalogue de gestes et de leurs significations possibles. Grâce à l'émergence de nouveaux dispositifs d'interaction dans le jeu vidéo et au succès des tablettes tactiles, le répertoire de gestes s'est en effet diversifié. Dans un article issu d'un projet de recherche commun ${ }^{6}$, Serge Bouchardon [2011] considère l'action du lecteur comme un "énoncé de gestes » (par exemple le glisser/déposer - drag and drop) qui prend lui-même une signification plus globale grâce à la combinaison au contexte. L'auteur propose un tableau synthétique de ces " énoncés de gestes » avec leurs " traits signifiants possibles », qui s'actualisent potentiellement dans la combinaison avec un texte ou une image. L'exemple analysé en profondeur dans l'article de Serge Bouchardon n'est pourtant pas textuel ; et la notion de " traits signifiants » que j'avais moi-même adoptée dans certaines publications

6. Projet « Signes et figures de la création numérique », initié en 2009, avec Philippe Bootz, Serge Bouchardon, Jean Clément et Alexandra Saemmer. Ouvrage en cours de préparation. 
[par exemple, 2011a], me paraît aujourd'hui trop inspirée de la sémiotique linguistique du texte ; or, une icône n'est décidément pas un mot.

Le texte alphabétique se caractérise par une relation en principe arbitraire entre les caractéristiques matérielles du mot et son potentiel d'action, dont la pratique partagée dans une société fonde les répertoires à la fois relativement stabilisés (permettant l'instauration d'une situation de communication) et dynamiques (permettant l'appropriation et l'expression individuelles).

La reconnaissance d'un signe iconique par le lecteur est, selon JeanMarie Klinkenberg [1996, 385], constituée par des processus d'intégration et de stabilisation d'expériences antérieures. Dans le champ du texte manipulable, le geste d'activation d'un hyperlien pourrait donc renvoyer à l'expérience d'appui sur des objets dans l'entourage immédiat du lecteur, que ces objets soient technologiques ou pas. Le rapport iconique d'analogie avec le référent d'expérience conserve des traits de l'« original », même s'il les reconstruit dans un matériau et à une échelle différents.

Dans le cadre de notre projet « Signes et figures $»^{7}$, nous avons élaboré l'hypothèse que certains enchaînements de gestes constituent donc le signifiant d'un signe iconique, que nous avons décidé d'appeler " unité sémiotique de la manipulation ». Comme je l'ai déjà dit plus haut, je suis bien évidemment consciente à quel point l'identification de telles « unités sémiotiques » est problématique : leur isolement du contexte de réception ne peut donc être que passager. Afin de sonder avec précision le rôle du geste couplé au texte à lire, cette étape me paraît néanmoins éclairante.

Je pars donc de l'hypothèse que dans les unités sémiotiques de la manipulation, l'articulation de gestes sur une zone interactive fait appel à des processus d'intégration et de stabilisation d'expériences antérieures. Le lecteur reconnaît, par exemple, l'unité " activer », présente dans la manipulation de beaucoup d'hyperliens et caractérisée par un geste d'appui et de relâchement bref et répétitif sur une zone manipulable, parce qu'il l'a déjà expérimentée maintes fois en appuyant brièvement sur des objets qui l'entourent : des interrupteurs, des boutons de commande... Il reconnaît de même l'unité " gratter », actualisée dans les jeux de grattage en ligne et formée à partir de gestes d'appui prolongés et répétés combinés à un déplacement de la souris ou du doigt sur un touchpad ou un écran tactile, parce qu'il a déjà gratté, raboté du bois ou dépoussiéré des surfaces. Et il a sans doute déjà appuyé de façon répétitive sur une sonnette et expérimenté 
ainsi l'unité « insister », actualisée dans certains jeux vidéo où le lecteur est censé « tirer » de façon répétée sur des objets ou des personnages.

Ces unités sémiotiques de la manipulation relèvent de l'icône parce que leur potentiel d'action ne se démarque guère des référents d'expérience. L'unité « activer » peut ainsi renvoyer à des idées de réactivité, de maîtrise, de déclenchement instantané. L’unité " gratter » peut renvoyer à des idées de nettoyage ou de façonnage progressifs. L'unité " insister » peut évoquer des idées d'urgence et d'acharnement compulsif. En verbalisant ainsi le "signifié iconique », il ne faut pourtant pas oublier, comme le formule Jean-Pierre Meunier [2006, 137], qu'il « n'est pas un objet pour la pensée consciente », mais plutôt " une forme avec laquelle le corps percevant entre en résonance mimétique ». D’une façon différente, autrement plus sensuelle que le texte, le caractère iconique d'une unité comme « activer » contribue à la constitution du potentiel d'action d'un hyperlien.

\section{« Irradiations iconiques » dans le texte manipulable}

Un processus que je propose d'appeler « irradiation iconique » agit dans tous les textes manipulables à partir du moment où un texte est à la fois lu et agi par le lecteur : le potentiel d'action de l'unité sémiotique de la manipulation se couple au potentiel d'action du texte à lire et peut souligner, préciser, renforcer ou alors déstabiliser celui-ci, formant des procédés rhétoriques qui anticipent sur des pratiques de lecture. Afin de donner dès maintenant un rapide aperçu des couplages possibles entre unités sémiotiques de la manipulation et textes alphabétiques, voici deux exemples.

Dans un épisode de l'hyperfiction Zeit für die Bombe ${ }^{8}$ déjà évoquée plus haut, le lecteur est invité à manipuler les mots « appuie sur le petit interrupteur " afin de déclencher (à l'instar d'un personnage de la fiction) le compte à rebours d'une bombe. Le recours aux unités sémiotiques de la manipulation me permet maintenant de mener une analyse plus précise de ce couplage rhétorique entre texte et gestes. Le mot "interrupteur » renvoie en effet à un outil, sur lequel nous effectuons habituellement le même enchaînement de gestes qui est sollicité par l'unité sémiotique de la manipulation « activer » sur support numérique. Dans ce cas précis, le lecteur a donc peut-être, au moins partiellement, l'impression de manipuler la chose interrupteur.

8. Susanne Berkenheger, Zeit für die Bombe, [en ligne] : <http://www.wargla.de/zeit.htm>. 
Figure 1. Extrait de l'hyperfiction Zeit für die Bombe de Susanne Berkenheger

Alors moi je comprends Iwan :

Est-ce que nous n'avons pas tous tendance à vouloir toujours appuyer

sur quelque chose, cliquer quelque part, afin de déclencher des

événements sans grand effort? C'est en effet la plus belle chose au monde.

Iwan, fais-le enfin,

$\underline{\text { appuie sur le petit interrupteur ! }}$

Original en allemand consultable à l'adresse <http://www.wargla.de/96Dollar. htm> (consulté le 18 mai 2012).

La spécificité de ce procédé rhétorique apparaît encore plus clairement lorsqu'on le compare à un autre hyperlien repéré sur Wikipédia. Dans un article consacré aux « disjoncteurs » électriques, le lecteur est également invité à activer le mot hyperlié " interrupteur ». Dans Wikipédia, compte tenu du fait qu'il s'agit d'une encyclopédie, le lecteur s'attend sans doute à une définition lorsqu'il active le mot « interrupteur ». Dans l'hyperfiction Zeit für die Bombe, lorsqu'il active le mot « interrupteur », il s'attend plutôt à ce que le compte à rebours se déclenche ou que la bombe explose.

Figure 2. Capture d'écran d'un extrait de l'article de Wikipédia consacré aux disjoncteurs

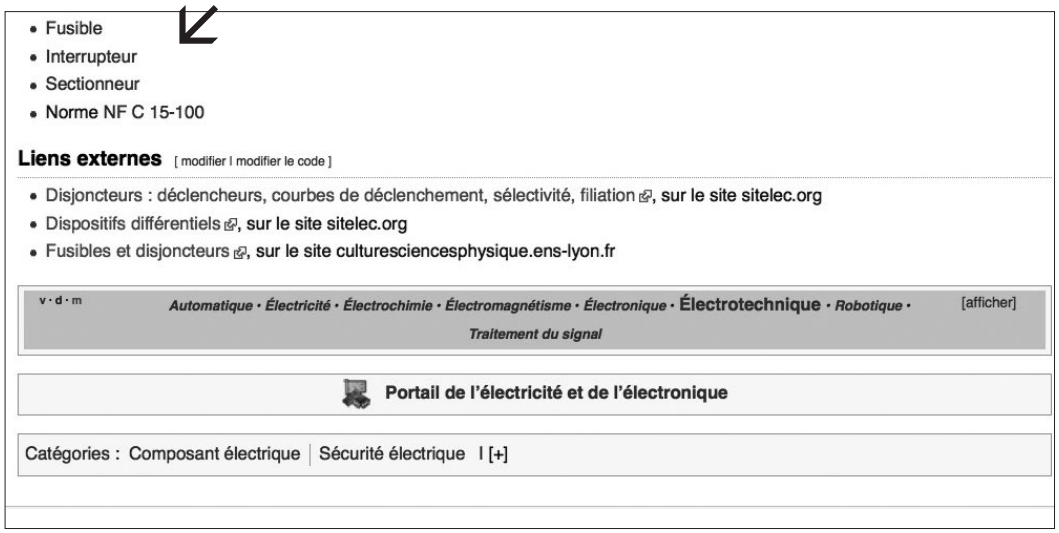

Consultable à l'adresse : < http://fr.wikipedia.org/wiki/Disjoncteur> (consulté le 18 mai 2012). La flèche ajoutée par mes soins sur la capture d'écran marque la zone manipulable. 
Dans Wikipédia, l'irradiation iconique agissant sur le mot « interrupteur » suscite certes potentiellement des attentes de réaction et de révélation immédiates, et procure un certain plaisir du geste sans pour autant rapprocher le mot hyperlié de la chose évoquée. Cette forme modérée d'irradiation iconique est de loin la plus fréquente sur Internet. Si le lecteur a, en revanche, dans Zeit für die Bombe, outre le plaisir du geste et le rapport d'analogie entre l'appui sur un interrupteur " réel » et l'appui sur l'hyperlien, l'impression d'activer la chose « interrupteur », c'est parce qu'un personnage de cette fiction est invité à effectuer le même geste au niveau de l'histoire racontée. C'est donc aussi en fonction des répertoires et du genre textuel que se crée, dans ce couplage entre texte et unité sémiotique de la manipulation, un simulacre de référent qui peut constituer un puissant facteur d'immersion.

Les effets de présentification et le plaisir immersif potentiellement suscités font même dire à certains que le lecteur se transforme lui-même en « poseur de bombes » [Simanowski, 1999 ; voir étude de la réception de cette hyperfiction au chapitre III, " Figures de la lecture et formesmodèles de la page-écran dans le discours narratif », p. 183]. La pratique de lecture anticipée dans ce couplage se fonde sur la présomption que le sujet accepte de s'abandonner à ce simulacre.

Les conséquences de cet abandon peuvent paraître à la fois fascinantes et aliénantes, rappelant des jeux vidéo où il faut pousser des portes par clic de souris, ou éliminer des adversaires avec le geste qu'on mobiliserait aussi en appuyant sur le déclencheur d'une arme automatique. Confronté à des simulacres de référent, le lecteur soit se réjouit, avec Hans Ulrich Gumbrecht [2010, 209], de " retrouver certaines choses du monde » dont l'univers médiatisé l'avait peut-être aliéné, et s'adonne sans retenue au sentiment d'intensité. Soit il se défend, avec Theodor W. Adorno [1963], de la « réceptivité passive » éventuellement engendrée par ces simulacres de référent, craignant l'évanouissement de sa capacité de réflexion critique.

La question des avantages et inconvénients de l'immersion se pose de façon particulièrement cruciale dès qu'un journaliste commence à expérimenter l'irradiation iconique (voir exemples dans le corpus présenté au chapitre III, « Figures de la lecture du texte numérique dans les discours informatif et argumentatif », p. 116). La sensualité des enchaînements de gestes peut en effet faire débat dès qu'elle est utilisée pour abolir la distance entre le lecteur et les événements rapportés. 


\section{Unités sémiotiques de l'animation}

J'en viens maintenant au texte animé. Comme c'est le cas pour les unités sémiotiques de la manipulation, je propose de considérer certains mouvements comme des "icônes » : des signes qui s’approchent par leur structure visuellement percevable d'une représentation de leur référent d'expérience. Dans le cadre de notre projet de recherche "Signes et figures... ${ }^{9}$, nous avons décidé de les appeler " unités sémiotiques temporelles » en référence aux unités du même nom dans le domaine sonore. Cette mise en relation entre mouvement et son peut surprendre et doit être explicitée.

Dès qu'on se penche sur le couplage entre mouvement et texte, que ce soit dans des bannières publicitaires ou dans la littérature numérique, la question se pose du potentiel d'action du mouvement. Comme dans le cas de la manipulation où l'on pourrait être tenté de considérer chaque micro-geste comme une icône potentielle, il faut se demander si chaque micro-phénomène visuel, par exemple une apparition ou une disparition au sein d'un clignotement, fait appel à des expériences déjà rencontrées, ou si c'est plutôt certains enchaînements de mouvements qui constituent l'icône. Il faut ensuite étudier si les caractéristiques visuelles seules comme l'enchaînement entre apparition et disparition - fondent l'unité de l'icône, ou si d'autres caractéristiques, comme le rythme, jouent un rôle important, voire prépondérant.

À partir d'une première étude comparative d'exemples dans la publicité en ligne (voir projet "Signes et figures... ») ${ }^{10}$, j'ai constaté que des mouvements aux caractéristiques visuelles fort différentes se trouvent parfois couplés aux mêmes textes et anticipent ainsi, de toute évidence, sur des pratiques de lecture semblables. Certains auteurs font par exemple clignoter le mot « soldes » de façon rapide et réitérée ; d'autres le font gonfler et rétrécir au même rythme ; d'autres encore le font changer rapidement et périodiquement de couleur. Ce qui rapproche ces mouvements, en dehors des différences dont je ne nie pas l'importance dans la communication visuelle, est le rythme rapide et la réitération. Une modélisation que j'ai effectuée à partir du mot " soldes » en le faisant clignoter, changer de couleur, gonfler et rétrécir au même rythme dans un même contexte (voir captures d'écran ci-contre et animations consultables en ligne), a donné $\mathrm{du}$ fondement à l'hypothèse, que ces trois mouvements mobilisent un

9. Projet « Signes et figures de la création numérique », op. cit. 10. Ibid. 
potentiel d'action semblable. Ce seraient donc plutôt les caractéristiques liées à la temporalité qui déterminent l'émergence du potentiel d'action d'un mouvement.

Figure 3. Le mot « soldes » change de couleur ou de taille, à un rythme rapide et de façon réitérée

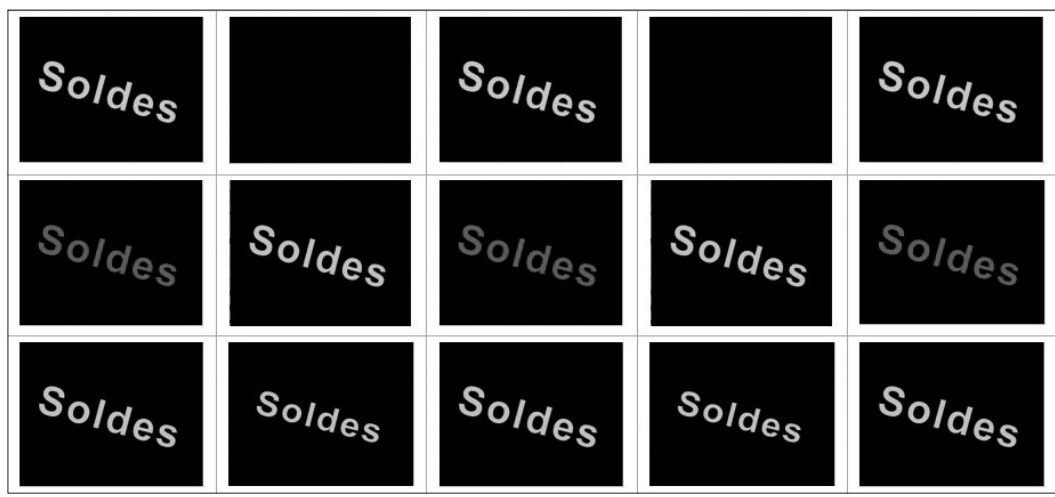

Modèles consultables aux adresses :

$<\mathrm{http}: / /$ www.alexandrasaemmer.fr/corpus/obsessionnel/soldes1.html>, $<\mathrm{http}: / /$ www.alexandrasaemmer.fr/corpus/obsessionnel/soldes2.html>, $<\mathrm{http}: / /$ www.alexandrasaemmer.fr/corpus/obsessionnel/soldes3.html>.

À partir de l'étude du corpus, il m'a été possible de regrouper d'autres mouvements ou enchaînements de mouvements qui présentent des détails visuels parfois assez divergents tout en mobilisant un potentiel d'action semblable.

Certains objets textuels disparaissent par exemple lentement, soit parce qu'ils s'effacent progressivement, soit parce qu'ils deviennent de plus en plus flous. Les deux mouvements sont caractérisés par une baisse progressive d'énergie.

Certains autres éléments textuels s'agrandissent progressivement, donnant l'impression de se rapprocher ; d'autres apparaissent en devenant de moins en moins flous; d'autres encore changent progressivement de position sur l'écran, voire le quittent vers le « hors-cadre ». Ce qui rapproche ces mouvements est le fait de décrire une évolution linéaire, constante et sans perte d'énergie. 
Une fois ces regroupements effectués, j'ai eu connaissance ${ }^{11}$ des recherches sur les unités sémiotiques temporelles menées depuis plusieurs années au MIM, un laboratoire de musicologie indépendant à Marseille. Ce laboratoire a identifié des unités sémiotiques temporelles (UST) dans la musique ${ }^{12}$. Des expérimentations empiriques [Frey, Poitrenaud, Tijus, 2010] laissent penser que les auditeurs reconnaissent ces unités grâce à un certain nombre de caractéristiques qui touchent souvent au rythme, à la réitération et à l'intensité sonores. Les noms attribués aux unités circonscrivent la présence de ces caractéristiques.

L’unité " obsessionnel » est ainsi constituée d'un enchaînement dont la répétition crée une pulsation rapide : par exemple, une même note de piano répétée indéfiniment. J'invite le lecteur à écouter un extrait sonore de l'« obsessionnel » sur le site Web du MIM ${ }^{13}$.

L'unité "sur l'erre » comporte une décroissance d'intensité sonore jusqu'à l'extinction. Dans l'exemple sur le site du MIM, le volume d'un son baisse progressivement jusqu'à devenir inaudible ${ }^{14}$.

La « trajectoire inexorable » est une unité à phase unique présentant une évolution linéaire, qui suggère que cette évolution continue indéfiniment. Dans le domaine musical, elle est exemplifiée par un son qui n'en finit pas de monter ${ }^{15}$.

Il suffit maintenant de visualiser les mouvements insistants d'apparition et de disparition, de changement de taille ou de couleur rapides du texte dans les exemples de bannières " soldes » modélisés plus haut, et d'écouter en même temps l'exemple sonore de l'« obsessionnel » où une note de piano est répétée indéfiniment et de façon insistante, pour être frappé par l'impression de synesthésie.

Il en est de même pour les animations où un texte s'efface progressivement jusqu'à disparaître, et pour l'exemple sonore du « sur l'erre » où une note de piano s'éteint progressivement jusqu'à devenir inaudible.

De même, un extrait de la "trajectoire inexorable », dans lequel un son n'arrête pas de monter, semble pouvoir entrer en correspondance forte

11. Grâce à la collaboration avec mon collègue Philippe Bootz autour du projet de recherche "Signes et figures de la création numérique », initié en 2009.

12. Laboratoire Musique et informatique de Marseille, "Les unités sémiotiques temporelles ", [en ligne] : < http://www.labo-mim.org/site/index.php?2008/08/22/44-liste-des-19-ust >.

13. « Obsessionnel », [en ligne] : < http://www.labo-mim.org/site/index.php?2008/08/22/36-obsessionnel >.

14. « Sur l'erre », [en ligne] : < http://www.labo-mim.org/site/index.php?2008/08/22/45-sur-l-erre >.

15. « Trajectoire inexorable », [en ligne] : < http://www.labo-mim.org/site/index.php?2008/08/22/28trajectoire-inexorable $>$. 
avec les mouvements de déplacement ou de rapprochement progressifs d'un objet visuel sur l'écran.

Dans le projet «Signes et figures... » ${ }^{16}$, nous avons donc élaboré l'idée que les UST constituent les unités d'une sémiotique temporelle qui peut être implémentée dans du son, du texte ou de l'image. J’ai pu identifier jusqu'alors quatre unités sémiotiques temporelles avec leurs correspondances dans le son et dans le mouvement (voir tableau des formes-modèles pro-référentielles du texte animé au chapitre III, p. 229). J'ai décidé de garder la terminologie élaborée par le MIM pour les nommer.

Comment expliquer la reconnaissance d'une unité sémiotique par le lecteur malgré les caractéristiques visuelles parfois si différentes : par exemple le clignotement, le changement de taille et le changement de couleur rapides qui feraient partie de la même unité appelée « obsessionnel » ? Cette reconnaissance semble encore fondée sur des processus de stabilisation d'expériences antérieures. Le lecteur reconnaît donc l'unité « obsessionnel » parce qu'il l'a déjà expérimentée en observant l'animation rapide et réitérée de certains objets dans son entourage, comme le clignotement de boutons d'alarme. Il reconnaît l'unité « sur l'erre » grâce à des expériences de disparition et d'effacement, par exemple l'observation d'une tâche d'eau qui s'évapore progressivement. Il a aussi expérimenté le déplacement sans perte d'énergie de certains objets caractéristique de la « trajectoire » à chaque fois qu'une voiture s'approchait ou traversait son champ de vision en décrivant un mouvement continu.

Le potentiel d'action des unités sémiotiques temporelles ne se démarque guère de ces référents d'expérience. L'« obsessionnel » peut renvoyer à des idées de danger et d'urgence. Le " sur l'erre » peut renvoyer à des idées de perte et de disparition définitives. Une « trajectoire inexorable » peut renvoyer à des idées de matérialisation rassurante, de révélation, de cohérence, de transformation sans perte de matière. Encore une fois, il ne faut pourtant pas oublier que l'icône n'est pas seulement un objet pour la pensée consciente, mais surtout « une forme avec laquelle le corps percevant entre en résonance mimétique » [Meunier, 2006, 137]. Grâce à son caractère insistant, l'obsessionnel semble ainsi pouvoir provoquer la capture de l'attention du lecteur, et cela indépendamment du texte précis auquel il est couplé. Voilà sans doute pourquoi ce mouvement est si fréquemment utilisé dans les bannières publicitaires sur Internet (voir

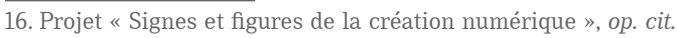


chapitre III, « Figures de la lecture dans le discours persuasif », p. 217) et dans la signalisation routière.

\section{Irradiations iconiques dans le texte animé}

Dans l'animation textuelle, une unité sémiotique temporelle se trouve couplée à des textes à lire et, en fonction de l'horizon d'attente du lecteur, entre avec celui-ci dans une relation de contiguïté plus ou moins forte. Cette relation, fondée sur une irradiation iconique de l'unité sémiotique temporelle sur le texte à lire, peut aller de la confirmation à la contradiction, constituant des figures de rhétorique qui anticipent sur les pratiques du lecteur. Notamment dans la publicité, les auteurs souhaitent souvent qu'une contiguïté forte s'établisse entre le potentiel d'action du texte et le potentiel d'action du mouvement, pour que le message s'en trouve renforcé.

Je reprends l'exemple du mot « soldes » qui clignote sur un site commercial. En France, la période des soldes est encadrée par la législation et s'étend sur un laps de temps assez court. La hâte avec laquelle certains consommateurs envahissent les grands magasins lors du premier jour de cette période montre bien qu'un sentiment d'urgence peut faire partie des répertoires partagés par le texte et le lecteur. L'iconicité du mouvement de clignotement renforce potentiellement ce sentiment d'urgence, lui donnant une matérialité que le texte seul ne possède pas.

Dans certains cas, l'irradiation iconique sur le texte est poussée à l'extrême de sorte que se crée un simulacre de référent. En guise d'exemple, on pourrait imaginer le récit d'une histoire d'amour où le mot " cœur » s'agrandit et se rétrécit de façon rapide et réitérée au milieu de la phrase : «Face à Julia, le *cœur* de Julien bat la chamade ». Le mouvement imite celui d'un cœur vivant. La distance avec le référent d'expérience est pourtant à la fois abolie et soulignée : le texte reste à tout moment alphabétique et ne se transforme jamais en objet, et le clignotement régulier n'imite que partiellement le battement d'un cœur vivant, forcément soumis à des irrégularités.

En outre, certains lecteurs familiarisés avec les dispositifs numériques pourraient interpréter ce couplage non pas comme une imitation du cœur humain, mais comme le signalement d'un hyperlien. Cette interprétation devient sans doute plus probable encore si le mot "*cœur* » animé se trouve sur un site médical consacré aux problèmes cardiaques, ou dans un article scientifique dédié aux progrès dans le domaine de la transplantation du cœur. Les répertoires, stratégies et annonces du texte entourant 
une animation textuelle influencent donc de manière décisive l'actualisation de ce procédé rhétorique. Les effets de présentification et d'immersion mobilisés par les unités sémiotiques temporelles, recherchés dans le domaine du jeu vidéo ou de l'hyperfiction, peuvent surprendre, voire déconcerter le lecteur s'ils se trouvent insérés dans un texte scientifique ou un article journalistique.

\section{Approches des formes-modèles de la page-écran}

Diverses formes-modèles qui ne relèvent pas des matérialités du texte, mais bordent et cadrent celui-ci sur la page-écran, anticipent également sur les pratiques de réception par le lecteur. Certaines d'entre elles ont émergé avec l'évolution des ordinateurs. Elles ancrent le texte dans les représentations socialement partagées du numérique. D’autres contiennent des allusions aux supports de lecture qui les ont précédés. Beaucoup de sites Web, mais aussi de nombreuses applications développées pour tablette numérique présentent en effet au lecteur des repères qu'il reconnaît parce qu'il les a d'abord rencontrés dans l'univers papier. Dans la presse en ligne, Jeanneret, Béguin, Cotte et al. [2003] constatent ainsi un balancement constant entre des formes qui manifestent la nouveauté du média, et d'autres qui ancrent le texte dans une série de formes reconnues et légitimées. L'identification de ces formes et la circonscription de leur potentiel d'action sont un enjeu important.

La première page-écran d'Inside - a Journal of Dreams, une hyperfiction d'Andy Campbel1 ${ }^{17}$, confronte le lecteur à la représentation d'un objet carré à l'aspect usé, facilement identifiable comme la quatrième de couverture d'un livre fermé. Le projet a été développé en 2004, avant l'arrivée des tablettes tactiles. Le lecteur est invité à interagir avec ce livre évoqué en cliquant sur des zones manipulables ou en activant des mots-clés comme "rotate » et "inverse », logés dans un menu à côté de l'objet. Une fois ouvertes par l'activation d'un ornement sur la couverture, les pages de ce livre, dont le fond imite la granularité du papier, se feuillettent par appui sur un bouton à côté des mots «next » et " prev » (pour previous) dans la marge.

17. Andy Campbell, Inside - a Journal of Dreams, [en ligne] $:<$ http://www.dreamingmethods.com/ inside/ >. 
Figure 4. Capture d'écran d'un extrait d'Inside - a journal of dreams par Andy Campbell

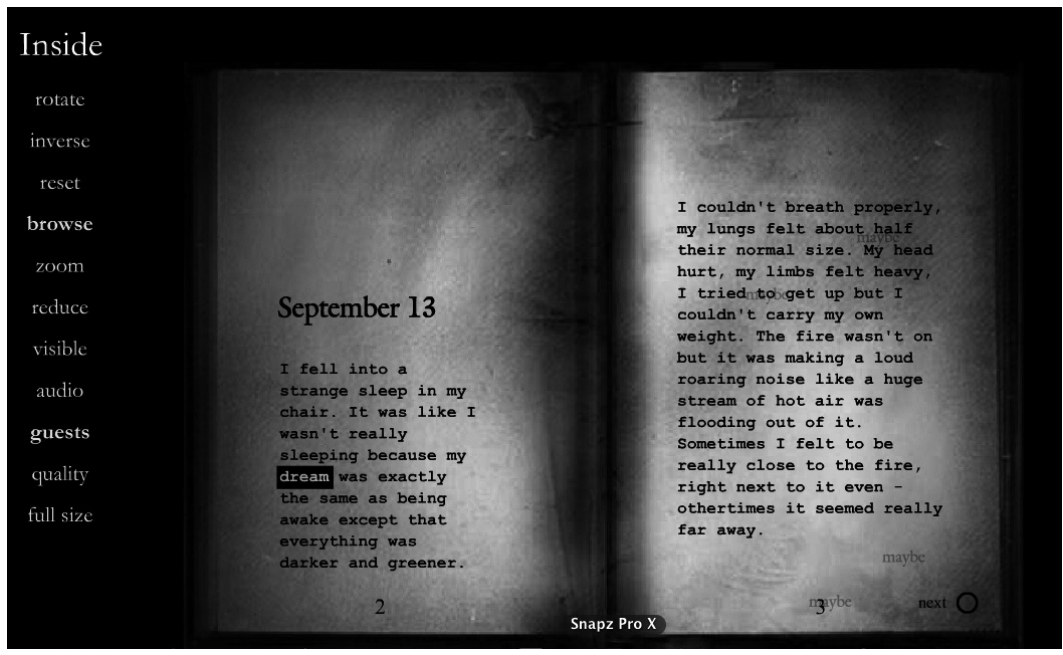

Consultable à l'adresse : < http://www.dreamingmethods.com/inside/ > (consulté le $1^{\mathrm{er}}$ mars 2012)

La représentation des pages suggère la possibilité d'un feuilletage qui, dès la couverture, est partiellement mis en échec parce que le geste sollicité relève de l'unité " activer », et non pas de l'appui-glissé qui est caractéristique du feuilletage papier ${ }^{18}$. Néanmoins, ce feuilletage, même incomplètement imité, rappelle celui des livres " non ergodiques » [Aarseth, 1997, 1], dans lesquels l'effort pour traverser le texte est « trivial, avec aucune responsabilité extra-noématique demandée au lecteur » [ibid.]. L'hyperlien imitant le feuilletage des pages peut donc être considéré comme une " forme-modèle » qui renvoie au livre papier, et anticipe peut-être sur un lecteur nostalgique des caractéristiques de ce support de lecture. La représentation de la granularité, du format et de l'irrégularité de la page, du volume et de l'épaisseur du livre convoque de même le « fantôme » du papier.

Cependant, plusieurs pages d'Inside comportent des hyperliens placés sur des mots du texte alphabétique, qui cette fois-ci exigent du lecteur

18. Dans des créations pour iPad comme Alice au pays des merveilles [Atomic Antelope, 2010], l'imitation du feuilletage de pages a été perfectionnée. Le lecteur passe de page en page par un geste d'appui-glissé, le même geste qu'il effectuerait en feuilletant un livre papier. Son geste s'accompagne même d'un léger bruit de papier froissé. 
un effort «non trivial », [Aarseth, 1997] étroitement associé aux supports numériques. Il en est ainsi de l'hyperlien posé sur le mot " page 2 (voir figure 4) inséré au milieu du feuillet. Le couplage entre le texte et l'icône préfigure un lecteur qui prend le risque de rompre le fil discursif, de jouer avec la disparition du texte géniteur et avec l'apparition d'un texte relié ; un texte relié qui, contrairement aux pages feuilletables, ne fait pas partie constituante de l'épaisseur du livre évoqué (le texte relié à cet hyperlien ne comporte pas de numéro de page). Lisant et manipulant le couplage entre le mot "dream" et l'unité " activer », le lecteur est invité à littéralement plonger dans le rêve du personnage principal. Ce couplage transforme donc, au moins partiellement, le texte en simulacre de référent - à condition que le lecteur déchiffre le mot hyperlié avant de cliquer.

Comme Milad Doueihi [2011], Yves Jeanneret [2008, 174] insiste sur le fait que la disparition effective de certaines unités de présentation comme la page, et la pérennité de la filiation sémiotique de certains éléments qui rappellent quand même la page, constituent un champ de tensions dont il faut prendre en compte la portée dans l'analyse du processus de réception du texte numérique.

Comme le proposent Bolter et Grusin [2000] à travers le terme "remediation”, les supports de la lecture papier se « reconnaissent » dans le numérique à travers un certain nombre de formes-modèles, mais se transforment aussi afin de répondre à l'évolution de l'horizon d'attente du lecteur. L'une des conséquences possibles de cette évolution est qu'un lecteur en 2012, éventuellement familiarisé avec la manipulation des " livres augmentés » pour tablettes, aborde Inside - a Journal of Dreams avec des expectatives bien différentes de celles des «premiers lecteurs » en 2004. Des formesmodèles qui, pour un lecteur en 2004, pouvaient éventuellement constituer des repères rassurants, peuvent être considérées comme anachroniques par les lecteurs récents. Au chapitre III, je reviendrai sur les formes-modèles de la page-écran et proposerai une typologie plus précise.

\section{Approches des matérialités du dispositif}

Bien avant que le lecteur entreprenne la lecture d'un texte, bien avant qu'il commence même à repérer les formes-modèles sur la page-écran, le « dispositif » est présent et anticipe sur les pratiques. Le terme « dispositif » est à la fois omniprésent dans les discours consacrés au numérique, et flou dans son usage. Jean-Paul Fourmentraux [2005, 31] recourt utilement 
à la racine du terme en rappelant que celui-ci met des contenus « à disposition » du public. Dans sa définition, les dispositifs sont composés d'« une avant-scène », c'est-à-dire d'une « interface » qui « inscrit la réception active du public » : cette interactivité va de l'activation d'hyperliens jusqu'à l'introduction de données. Deuxièmement, le dispositif comporte la "scène matricielle », composée de divers éléments incrémentaux qui viennent nourrir une création numérique. Troisièmement, elle inclut les « coulisses », c'est-à-dire le programme informatique et les applications.

La résolution de l'écran, la luminosité et l'affichage des couleurs semblent fortement affecter la lecture du texte numérique. Dans une étude par questionnaire menée à l'université Lyon 2 en 2006 auprès de 600 étudiants $^{19}$, les sujets interrogés mentionnaient comme l'un des obstacles majeurs à la lecture numérique la fatigue oculaire provoquée par les caractéristiques de l'écran. La position assise exigée par la manipulation d'un ordinateur de bureau peut également contribuer à cette impression de fatigue physique [voir Jeanneret, Béguin, Cotte et al., 2003]. Les dispositifs mobiles y remédient éventuellement, permettant au lecteur de changer facilement de position : la lecture numérique peut désormais s'effectuer au lit, dans le train, dans un parc ou dans la rue. La taille de la « lucarne » ouverte sur le texte numérique est pourtant inversement proportionnelle à sa mobilité. Il n'est donc pas certain que le téléphone portable facilite la lecture d'un texte long.

Les coulisses du dispositif, c'est-à-dire les programmes informatiques, sont une autre composante à prendre en compte dans une rhétorique du texte numérique. Comme le soulignent Davallon, Després-Lonnet, Jeanneret et al. [2003, 23], on ne peut produire un texte à l'écran sans ces outils d'écriture situés en amont qui constituent son " architexte » [voir aussi Jeanneret, Souchier 1998] : « les outils qui permettent l'existence de l'écrit à l'écran et qui, non contents de représenter la structure du texte, en commandent l'exécution et la réalisation. Le texte naît de l'architexte qui en balise l'écriture ». Les concepts d'" énonciation éditoriale » et d'« architexte » soulignent le fait que l'« image du texte » n'est donc décidément pas anticipée par l'auteur seul, mais aussi par de multiples autres

19. Cette enquête, dirigée par Claire Bélisle au LIRE-CNRS, a été mise en place auprès d'étudiants universitaires en 2006 pour étudier dans un premier temps leur usage des encyclopédies en ligne. Un certain nombre de questions concernant leur rapport général au support numérique et leurs habitudes de lecture ont également été posées. Plus de 600 étudiants ont répondu à un questionnaire en ligne sur le bureau virtuel de l'université. Synthèse et analyse de cette étude consultable à l'adresse < http://lire.ish-lyon.cnrs.fr/spip.php?rubrique88 >. J'ai contribué à la mise en place, à l'évaluation et à la valorisation de ce projet de recherche. 
instances : traditionnellement le typographe, et plus récemment les éditeurs de logiciels, les programmeurs d'outils et les inventeurs d'algorithmes.

Comme l'explique Emmanuël Souchier [1998, 142], un texte est donc « le creuset d'une énonciation collective derrière laquelle s'affirment des fonctions, des corps de métiers, des individus... Et où fatalement se nouent des enjeux de pouvoir ". Cette énonciation collective s'exprime " à travers des marques d'énonciation éditoriale qui entretiennent un rapport "dialogique" avec l'histoire, l'histoire de l'art, les pratiques sociales ». Se pencher sur ces marques équivaut selon Yves Jeanneret et Emmanuël Souchier [2005, 3] à se demander non seulement « comment la sémiotisation du texte s'opère dans les processus matériels de sa mise en forme », mais à étudier en particulier les "médiations éditoriales, effectives et puissantes, qui s'opèrent par l'informatique, l'interface, le réseau et le logiciel ». Les logiciels utilisés pour l'écriture numérique entendent effectivement régir l'image du texte, et incarnent à travers les propositions de mise en forme une certaine conception du texte et de sa lecture soutenue par des logiques industrielles.

La gamme des outils utilisés pour donner un cadre éditorial au texte numérique va des logiciels de construction de pages Web comme Dreamweaver jusqu'aux systèmes de gestion de contenu. Comme l'ont montré certains chercheurs [voir parmi d'autres la thèse en cours d'Odile Farge ${ }^{20}$ et Saemmer, 2010a], l'écriture numérique a toujours été façonnée par les outils-logiciels qui, tout en facilitant l'écriture numérique aux non-programmeurs, leur proposent (voire leur imposent) des cadres de conception. Plus que jamais, les systèmes de gestion de contenu induisent cependant une séparation entre énonciation discursive et énonciation éditoriale. Ils proposent des modèles de pages qui incarnent une certaine idée de la lecture numérique : la présence d'un menu, par exemple, anticipe sur le besoin du lecteur d'accéder à une vue d'ensemble sur les contenus ; le cadre étroit de la fenêtre de contenu anticipe sur l'idée qu'il faut éviter des lignes de texte trop longues qui pourraient fatiguer l'œil.

Certes, le programme d'un texte numérique reste généralement caché au lecteur. Non seulement l'outil d'écriture qui a aidé l'auteur à structurer la page-écran, mais aussi le code informatique lui-même contiennent pourtant des représentations : la balise nofollow, insérée dans le programme des pages-écran de certains journaux en ligne, empêche par

20. Odile Farge, "Rhétorique de la conception : pour une prise de conscience des stratégies de l'outil de création. Proposition d'une typologie de postures d'auteurs », thèse soutenue le 8 décembre 2014 à l'université Paris 8, dirigée par Alexandra Saemmer. 
exemple le référencement d'un hypertexte externe par les moteurs de recherche, et reflète une représentation de la concurrence que l'autre texte constitue pour le journal.

Un élément important des matérialités du dispositif est aussi la vitesse de calcul de l'ordinateur. Philippe Bootz théorise et explore depuis 1990 ce qu'il appelle la " labilité du dispositif numérique ». En effet, des textes animés réalisés dans les années 1980 passent aujourd'hui à l'écran avec une vitesse qui rend leur déchiffrement impossible.

J'ai moi-même théorisé cette labilité du dispositif à partir de deux expériences clés. La première date de 2004 et résulte de la manipulation du numéro 12 de la revue Alire sur CD-ROM [éd. Bootz, 2004]. Lors d'une conférence, le menu interactif de cette revue a été présenté au public. Ce menu s'était affiché sous forme de rectangles traversant l'écran. La vitesse d'animation des éléments permettait de les manipuler avec aisance. Une fois le CD-ROM acquis, j'ai essayé de l'activer sur un ordinateur récent. À ma grande surprise, il était devenu difficile de manipuler les rectangles du menu, car ils traversaient l'écran à trop grande vitesse.

Une deuxième expérience s'est déroulée dans un cadre pédagogique : après avoir effectué une analyse détaillée de l'animation textuelle The Dreamlife of Letters de Brian Kim Stefans ${ }^{21}$ [voir Saemmer, 2008], je voulais présenter cette analyse à mes étudiants. J'ai donc affiché par vidéoprojection un extrait de cette œuvre, où un mot bouge de façon rapide et réitérée dans un contexte stable. Sur l'ordinateur récent utilisé pour cette projection, ce mouvement certes subtil, mais sur lequel j'avais fondé l'une de mes principales hypothèses d'interprétation, avait pourtant disparu : il passait trop vite sur l'écran pour être encore perceptible. Seul l'affichage du programme où le mouvement du mot était effectivement toujours inscrit me permettait de prouver aux étudiants l'existence de cette animation.

Le potentiel d'action des figures rhétoriques du texte numérique et des formes-modèles de la page-écran peut donc non seulement s'actualiser de façon différente d'un lecteur à l'autre (comme c'est le cas pour tout texte), mais d'un dispositif numérique à un autre. Les conséquences de cette observation sont considérables, autant pour l'interprétation du texte numérique qui se conçoit comme une pratique partagée entre lecteurs, que pour les questions de préservation et l'intégration de l'éphémère dans les pratiques de création.

21. Brian Kim Stefans, The Dreamlife of Letters, [en ligne] : < http://collection.eliterature.org/1/ works/stefans_the_dreamlife_of_letters/dreamlife_index.html $>$. 


\section{LA RHÉTORIQUE DE LA RÉCEPTION : SYNTHĖSE DES ASPECTS THÉORIQUES}

La rhétorique du texte numérique, dont je dessine les contours dans ce livre, considère la lecture comme une rencontre entre l'anticipation des pratiques par le texte numérique, et les imaginaires individuels et socialement partagés du lecteur qui préfigurent et actualisent ces anticipations. Il s'agit d'analyser comment le texte numérique à lire, à regarder et à manipuler, cadré dans une page et ancré dans son dispositif, anticipe sur les pratiques. Le relevé des imaginaires et attentes à l'aide d'études empiriques permet de circonscrire comment ce potentiel d'action du texte est actualisé comme pratique sociale de coconstruction du sens par les concepteurs et les lecteurs.

L'identification des procédés rhétoriques du texte numérique et des formes-modèles de la page-écran n'est pas qu'un enjeu de recherche théorique. Une connaissance des procédés argumentatifs, persuasifs, narratifs et poétiques du texte numérique sur la page-écran peut, d'une part, aider tout lecteur à développer des pratiques de lecture complexifiées. D'autre part, l'auteur de textes numériques pourra s'inspirer des typologies proposées pour diversifier ses pratiques d'écriture, et mieux tirer profit des potentiels que la rhétorique du texte numérique met à sa disposition.

Il lui sera ainsi possible de non seulement confirmer des informations par hyperlien, mais de proposer une mise en relation dialogique d'arguments et de contre-arguments. De même, l'animation textuelle pourra certes souligner le message du texte alphabétique, mais également anticiper sur un renversement ironique de celui-ci. Beaucoup d'auteurs expérimentent déjà avec les procédés rhétoriques du texte numérique sans toutefois disposer d'une vue d'ensemble sur le champ des possibles. Or, il me semble qu'une telle vue d'ensemble facilite l'expérimentation créative.

Je propose d'appeler les potentiels d'action du texte numérique " figures de la lecture ». Chaque figure actualise des imaginaires individuels et socialement partagés : c'est ainsi qu'elle préfigure une place pour le lecteur. Le potentiel d'action du texte numérique ne peut donc être analysé sur la seule base d'un relevé des procédés rhétoriques qui seraient immanents au texte. L'analyse doit prendre en considération les attentes potentielles du lecteur, ses habitudes et imaginaires. Les typologies des figures de la lecture du texte numérique et des formes-modèles de la page-écran proposées au chapitre III seront ainsi informées par les études empiriques sur les pratiques présentées au chapitre II et ne peuvent être 
présentées sans elles. Sur l'actualisation des formes et figures du texte numérique dans une situation de réception précise, cette méthodologie ne peut énoncer que des hypothèses.

Les figures de la lecture sont appelées ainsi parce qu'elles préfigurent les attentes et les pratiques du lecteur à travers les procédés rhétoriques du texte numérique. Dans le texte hypertextualisé, ces figures reposent sur différentes relations de contiguïté entre les textes associés par un hyperlien, sur l'ordre des arguments présentés (ce qui est donné à lire dans le texte géniteur contenant l'hyperlien, et ce qui est proposé dans le texte relié), sur les effets d'annonce dans le texte géniteur, sur les éventuelles reprises du texte relié dans le texte géniteur.

Chaque hyperlien est par ailleurs un élément manipulable qui implique le corps du lecteur. Il arrive aussi que des textes s'animent sur les dispositifs de lecture numériques : un mouvement se trouve dans ce cas couplé aux lettres, irradiant le potentiel d'action du texte à lire. La méthodologie proposée permet de circonscrire la part sensible de la lecture d'un hyperlien ou d'une animation à travers la notion d'iconicité. Elle se fonde sur l'hypothèse que certains enchaînements de gestes (mobilisés par exemple dans le clic-relâchement sur un hyperlien), et certains enchaînements de mouvements (mobilisés par exemple dans le clignotement) constituent des signes iconiques appelés unités sémiotiques temporelles et unités sémiotiques de la manipulation.

Le rapport iconique est fondé sur des processus d'intégration et de stabilisation d'expériences antérieures [Klinkenberg, 1996, 193]. Les potentiels d'action de ces unités ne se démarquent donc guère de leurs référents d'expérience : le clic-relâchement dans l'activation d'un hyperlien peut rappeler la manipulation d'un interrupteur sur un appareil électrique, et renvoyer à des idées de déclenchement et de réactivité immédiats. Néanmoins, le signifié iconique n'est pas qu'un objet pour la pensée consciente.

Il en est de même pour certains " énoncés de mouvements » mobilisés dans le texte animé. Un clignotement renvoie potentiellement à l'expérience d'objets clignotants dans l'entourage immédiat du lecteur (par exemple, des boutons d'alarme). Le rapport iconique d'analogie avec le référent d'expérience conserve quelques traits de l'original, même s'il les reconstruit dans un matériau différent. Le clignotement peut ainsi renvoyer à des émotions de stress, d'urgence et d'excitation tout en faisant autrement appel au corps du lecteur que le texte à lire.

Couplées à du texte, les unités sémiotiques de la manipulation et les unités sémiotiques temporelles peuvent, grâce à leur caractère référentiel, 
agir sur le lecteur sans qu'il s'engage dans le déchiffrement du texte. En cas de déchiffrement, les unités sémiotiques irradient potentiellement le texte et constituent des figures de la lecture qui confirment ou renforcent, troublent ou contredisent les répertoires de celui-ci, en fonction des attentes du lecteur.

Wolfgang Iser appelle « lieux d'indétermination » [1995, 299] les décohérences dans le texte qui mettent au défi les attentes du lecteur. Le terme Leerstellen (d'une façon insuffisante traduit par « vides »), utilisé par Iser, qualifie plus radicalement encore ces endroits qui sollicitent l'imagination du lecteur de façon particulière. Iser postule que la présence de lieux d'indétermination est indispensable pour la réussite de tout acte de communication. Avec ce postulat, il critique en outre certaines applications de la théorie shannonienne de l'information [Shannon, 1948] à la communication humaine ${ }^{22}$. Tout en employant parfois le terme d' « écart ", Iser constate par ailleurs l'échec de la «stylistique de la déviation » pratiquée par les représentants d'un structuralisme immanentiste. Il affirme que l'écart ne peut plus se rapporter exclusivement à une norme stylistique postulée, mais doit être situé par rapport à ce qu'il appelle des «normes d'attente » : des normes qui se rapportent aux « traditions socioculturelles » d'un public (167).

Si l'on transpose cette affirmation sur le terrain de l'hyperlien, il apparaît que la présentation de « faits » dans le texte géniteur et la confirmation de ces faits par le texte relié, dans un contexte de réception journalistique, répondent aux attentes de nombreux lecteurs. La juxtaposition d'arguments et de contre-arguments par un hyperlien dans le même contexte, en revanche, peut mettre au défi les attentes des lecteurs, d'autant plus si la présentation de contre-arguments n'est pas annoncée dans le texte géniteur. Une telle juxtaposition préfigure un arrêt transitoire de cette course effrénée vers le nouveau qui est souvent considérée comme caractéristique de la lecture numérique.

Une pratique interprétative permettrait, lors de cet arrêt momentané, d'élaborer un « montage » [Citton 2010, 37] qui remédie au moins partiellement au sentiment d'incertitude provoqué par le lieu d'indétermination. En effet, une mise au défi des attentes du lecteur peut parfois se résoudre par un " coup de force ", pour reprendre la terminologie de Bertrand Gervais $[1998,18]$ : par l'irruption d'une hypothèse qui assure un certain seuil de compréhension en «meublant » le lieu d'indétermination.

22. Comme le montre Claude Baltz [1999] dans son ouvrage Information : Shannon en Questions. Retour Sur un Concept Majeur, ces interprétations sont évidemment réductrices. 
Toute interprétation repose, comme l'indique Gilles Deleuze, d'abord sur une " soustraction » [1982]. Le lecteur élit certains éléments au statut de pertinents. Mais il hésite aussi, face à un lieu d'indétermination, reprend les contours de l'objet, lui superpose différentes hypothèses. Il fait ensuite son choix, effectuant " une espèce d'intégration de toutes les petites réactions cérébrales naissantes » afin d'arriver à un montage « totalisant », comme le résume Yves Citton [2010, 56].

Les figures de la lecture telles qu'elles sont définies et identifiées ici ne se fondent pas sur le postulat systématique d'un lieu d'indétermination, comme c'est le cas pour certaines définitions « classiques » de la figure de rhétorique [voir Quintilien, éd. 1987]. Alors que Tzvetan Todorov [1965, 300] affirme ainsi que " les effets de style ne pourraient exister s'ils ne s'opposaient à une norme, à un usage établi », François Rastier [2001, 35] s'insurge contre ces "pures constructions » que constituent pour lui la norme et l'écart, avançant que « le sens n'étant pas immanent au texte, mais à ses pratiques d'interprétation, il doit être rapporté à elles ». Épousant cette perspective, la rhétorique du texte numérique étudie les positions offertes par les lieux d'indétermination, afin d'examiner jusqu'à quel point l'imagination peut se déployer lorsque, par exemple, un manque de contiguïté logique entre texte géniteur et texte relié plonge le lecteur dans un état d'incertitude. Elle s'intéresse pourtant aussi aux figures de la lecture qui offrent la jouissance d'une satisfaction des attentes.

Comme le formule Yves Citton [2010, 29], toute connaissance relève d'une interprétation, car elle considère son objet à partir d'un point de vue. Un objectif important de la rhétorique du texte numérique est de faire émerger ces points de vue. Interprétant comment et pourquoi deux textes se trouvent reliés par un hyperlien, prenant en compte l'horizon d'attente partagé par les lecteurs à un moment donné dans une société, émettant des hypothèses sur le potentiel d'action des gestes et des mouvements couplés à certains textes numériques, la rhétorique tente de déconstruire les messages circulant dans la société, afin de montrer à quel point les significations sont d'une part anticipées par le texte, et d'autre part culturellement enracinées et socialement négociées. 


\section{CHAPITRE II FIGURATIONS DE L'HORIZON D'ATTENTE EXTRA-TEXTUEL}




\section{FIGURATIONS DE L'HORIZON D'ATTENTE EXTRA-TEXTUEL}

$\mathrm{L}$ e potentiel d'action d'un texte numérique se déploie dans la rencontre avec l'horizon d'attente à la fois individuel et socialement partagé du lecteur ; mais il préfigure aussi cet horizon d'attente. Par le terme « horizon d'attente », Hans Robert Jauss désigne ainsi deux composantes du texte : l'horizon « intra-textuel » tel qu'il est modélisé par les répertoires et les procédés rhétoriques du texte, et l'horizon « extra-textuel » tel qu'il est nourri à la fois par les centres d’intérêt, valeurs et références individuels, les normes, croyances, imaginaires et attentes socialement partagés $\mathrm{du}$ lecteur. Convaincu comme Roland Barthes qu'il faut observer la circulation du texte au sein de la société, Hans Robert Jauss conseille de ne pas s'en tenir à la reconstruction de l'horizon d'attente intra-textuel, mais de prendre en compte l'analyse des attentes dans le milieu social. Hans Robert Jauss s'est ainsi tourné vers une pensée communicationnelle du texte, ouverte sur le recueil de données auprès de lecteurs.

Le texte est considéré par les théories de la réception comme un processus, dont la vie sociale ne peut être étudiée qu’à travers ses concrétisations historiques successives. Il est évidemment impossible de recueillir tous les éléments constituant l'horizon d'attente extra-textuel. Si l'on veut néanmoins évaluer à quel point un texte numérique conforte ou défie les attentes du lecteur à travers les procédés rhétoriques qu'il mobilise, il faut essayer de circonscrire avec le plus de précision possible cet horizon d'attente. Il s'agit donc dans ce chapitre de reconstruire, au moins partiellement, l'horizon d'attente des «premiers lecteurs » [Jauss 2010, 50], et/ou des lecteurs à d'autres moments de la vie sociale d'un texte numérique, afin de pouvoir faire émerger dans la troisième partie le potentiel d'action des procédés rhétoriques identifiés à partir de corpus. Émergera ainsi une typologie de «figures de la lecture » du texte numérique fortement ancrée dans les pratiques.

Les représentations et les attentes du lecteur constituent des « interprétants globaux », des « noyaux d'appartenance » [Boutaud, Veron, 2007, 19], des croyances et connaissances partagées qui amènent un lecteur à approcher un texte numérique d'une certaine façon. J'ai précédemment 
parlé d'imaginaires, je propose maintenant de les appeler plus précisément « figurations".

De nombreuses études ont été menées ces dernières années sur les pratiques de lecture numériques. J’ai déjà cité l'étude par questionnaire conduite en 2006 à l'université Lyon 21, lors de laquelle nous souhaitions recueillir des informations sur la lecture numérique pratiquée par les étudiants en sciences humaines. Même s'il est difficile de tirer à partir des déclarations recueillies des conclusions sur les pratiques de lecture réelles, cette étude révèle des tendances. Elle sera croisée avec des études plus récentes.

Pour ce qui concerne les figurations du dispositif de lecture numérique et de l'Internet, je recourrai aux analyses proposées par Patrice Flichy [2001, 2003] et Dominique Cardon [2009, 2010], à des études exploratoires menées sur les représentations dans des discours officiels [Labelle, 2001] et dans la presse [Laborde, 2010]. Quant aux figurations de l'hypertexte, de l'hyperlien et de leur lecture, je puiserai des éléments dans des études empiriques et de nombreux discours de chercheurs. M'intéresse plus particulièrement la question de savoir quelles figurations de l'hyperlien ont tendance à être considérées comme des " normes d'attente ». Une étude exploratoire menée en 2011 à l'université Paris 8 me permettra de comparer les discours souvent normatifs sur l'hyperlien et sa lecture aux appréhensions et attentes de jeunes lecteurs à l'heure actuelle.

Afin d'étudier le potentiel d'action d'un texte numérique, il est également nécessaire de prendre en compte les figurations associées aux genres textuels. Je ne me pencherai pas ici sur l'influence des milieux socioculturels, de l'âge ou du sexe sur l'émergence de ces figurations, renvoyant le lecteur vers les nombreuses études à perspective sociologique (voir la synthèse de Jean-François Hersent [2000] ; les résultats des études concernant les «pratiques culturelles des Français » entre 1973 et 2008 par le ministère de la Culture et de la Communication ${ }^{2}$; l'ouvrage de Christophe Evans, Lectures et lecteurs à l'heure d'Internet : livre, presse, bibliothèques, 2011).

Il faudra par ailleurs garder à l'esprit que les figurations du dispositif, du texte et de la lecture évoluent et se transforment avec le temps, ce qui oblige le chercheur à renoncer à toute prétention à une modélisation transhistorique. En même temps, c'est précisément cet aspect évolutif qui fonde, comme le formule Wolfgang Iser [1995, 189], la « créativité de la

1. Voir note 19, p. 60 .

2. Enquêtes sur les "Pratiques culturelles des Français ", ministère de la Culture et de la Communication, depuis 1973, [en ligne] : < http://www.pratiquesculturelles.culture.gouv.fr/ >. 
réception " qui fait que toute interprétation constitue un processus où différents lecteurs, à différents moments, s' « inter-prêtent » des idées [Citton, 2010, 37] et participent au dynamisme transhistorique du texte.

\section{FIGURATIONS DE LA LECTURE NUMÉRIQUE}

Que n’a-t-il pas été dit et écrit sur la lecture numérique ! Depuis les débuts de l'informatique, et de façon insistante depuis la mise en œuvre de l'Internet, cette pratique a été investie de toutes sortes de figurations, qui vont de l'imitation de l'acte de lecture par la machine jusqu'à l'impossibilité déclarée de la lecture numérique faute de repères adéquats. « Fragmentaire », « inconfortable », « fluide », « impatiente », « sélective », " active ", « amnésique », " pressée », " sociale », " informationnelle », « fatigante » et « ludique » ne sont que quelques mots-clés revenant souvent dans les discours, qu'ils soient de source académique, avancés dans le cadre d'analyses sur corpus, recueillis auprès de lecteurs dans le cadre d'études empiriques ou relevés dans la presse. L'espoir d'une démocratisation de l'accès aux textes grâce à la numérisation, les craintes que ces textes ne trouvent pas pour autant de lecteurs, les louanges faites aux supports constamment renouvelés promettant un confort toujours plus grand, et l'observation que la jouissance du dispositif prend parfois le pas sur le plaisir de la lecture, se croisent sans toujours se rencontrer.

\section{Projection de figurations de la lecture papier}

Une première approche de la lecture numérique consiste à y projeter des figurations de la lecture papier. Certaines formes-modèles sur la pageécran soutiennent ces figurations en imitant le dispositif du livre ou du magazine papier (voir l'exemple de l'hyperfiction Inside - A Journal of Dreams cité plus haut). Elles soulignent, en creux, ce qui manque : l'odeur et l'épaisseur de la page, du volume... Une tablette conservera en effet toujours le même cadre, peu importe le volume textuel qui s'y actualise : qu'il s'agisse de la Recherche du temps perdu de Marcel Proust (des milliers de pages) ou de C'est tout de Marguerite Duras (quelques dizaines de pages), le même poids s'impose à la main et à l'œil. Beaucoup de sujets (par exemple, dans l'étude de 2006) insistent donc sur le fait que le volume réel du texte leur manque sur les dispositifs numériques, et ils regrettent la disparition de repères spatiaux. 
Derrière l'évocation du volume surgit une problématique de la lecture numérique fréquemment considérée comme centrale, et qui se trouve renforcée sur les tablettes qui limitent les possibilités du multifenêtrage (voir l'interface de la tablette Apple) : l'absence matérielle du texte avant le geste de manipulation qui permet de tourner la page, et la disparition du texte après le geste de manipulation effectué. L'incertitude de la suite, au sens le plus matériel du terme, semble à la fois l'un des principaux atouts de charme du texte numérique, et une source potentielle d'inquiétude et de désorientation. L'hyperlien, cet opérateur de l'effet d'absence-présence du texte numérique, s'est donc souvent trouvé en première ligne des critiques.

Thierry Baccino [2011, 63] doute que la lecture puisse être aussi " attentive » et «profonde » que celle sur papier. L'hyperlien risque de provoquer la perte de l'objectif initial de lecture, affirme l'auteur : « il y a de fortes chances que vous soyez distraits par une information qui n'a peut-être rien à voir avec la notion recherchée ». Le sujet n'arriverait plus à établir de la cohérence entre les passages lus. Alain Giffard craint un « abandon aux flux des relances médiatiques, au détriment de la lecture soutenue » [2011, 72]. Frommer [2011] et Desrichard [2011] présument que le toucher et le regard prennent le pas sur la compréhension et le sens. « Je ne sais pas lire une œuvre sur le Web... On n'a pas le même type de concentration sur l'écran », observe Barbara Cassin [2009, <http://www. telerama.fr/techno/internet-rend-il-bete,45486.php>].

Lors de l'étude menée à l'université Lyon 2 en 2006 (voir note 19, p. 60), nous avons constaté qu'une majorité d'étudiants considéraient en effet la lecture numérique comme problématique. Seulement $17,3 \%$ des sujets ont indiqué lire un texte entièrement sur écran. $37,3 \%$ ont dit l'enregistrer pour le lire "plus tard ». Et presque $50 \%$ des sujets ont affirmé l'imprimer systématiquement. 18,6\% des étudiants ont évoqué comme cause principale la «fatigue ». La lecture sur écran a été désignée comme une expérience " douloureuse ", qui " abîme les yeux ». Certains étudiants sont allés jusqu’à employer des termes comme " horrible », " abominable », " insupportable ", " une corvée », et l'un d'entre eux a même qualifié la lecture numérique de " contre nature ». "Il manque des éléments, sensations, sonorités propres à la lecture papier ", peut-on lire dans les questionnaires rendus, et : " un texte sur écran n'a pas de vécu comme un vrai livre ». Un étudiant a trouvé une formule poignante pour résumer ses réticences : "Le texte sur support numérique n’a pas d'âme, car celle-ci réside dans le papier ». Certains ont exprimé le regret de ne pas toujours bien comprendre ce qu'ils lisent. Ils ont déploré le côté « superficiel » de 
la lecture sur écran, le " risque de perdre le fil ». Ils ont affirmé que l'on est « moins impliqué dans ce qu'on lit».

Des réticences semblables ont été exprimées dans une étude de 2010, menée certes dans des conditions différentes, en collaboration avec l'éditeur Springer à l'université de Californie et auprès de 2569 étudiants [Li, Poe, Potter et al., 2011]. Beaucoup de lecteurs semblent en effet éprouver une pression temporelle qui leur fait parcourir le texte numérique dans tous les sens [voir aussi Jeanneret, Béguin, Cotte et al., 2003, 125]. Ils ont l'impression de mal résister à l'offre de découvertes qui se cache potentiellement derrière chaque hyperlien, chaque image, chaque enseigne clignotante. La lecture numérique est une expérience «frustrante » et " déstabilisante ». La « lecture réflexive » du « livre papier » demeure le contre-modèle le plus convoqué.

Comme il est difficile de lire un texte numérique tel un texte papier, certains lecteurs prennent la décision d'abandonner la lutte : « La lecture numérique, avec ses possibilités (ses nécessités) d'intermissions, de ruptures, de digressions, de dilettantismes, de sauts, d'interruptions, etc., n'a d'autre ambition que de cacher l'ennui de la vie moderne, vidé d'événements ", regrette Yves Desrichard : "Qu'à cela ne tienne, on s'en tiendra égoïstement à cet "espace protégé" évoqué par H. Rosa, à cette "oasis de décélération" délibérément préservé - pour le "monde vécu" des plus vieux, le livre, le livre papier » [2011, 62].

\section{La lecture-« zapping »}

Une deuxième figuration récurrente associe la lecture numérique à des pratiques de zapping. Un large consensus s'est formé ces dernières années quant à la rapidité accrue avec laquelle le lecteur traverse le texte numérique [voir par exemple Bélisle (introduction de Lire dans un monde numérique), 2011, 39 ; Jeanneret, Béguin, Cotte et al., 2003, 123 ; Vandendorpe, 2011, 54-55]. Cette pratique de lecture est fréquemment comparée au zapping télévisuel à cause de son caractère interactif : l'activation d'un hyperlien ne rappelle-t-elle pas en effet la manipulation d'un bouton de télécommande ? La réflexion sur les conséquences de cette pratique, en revanche, divise les esprits. Petrucci [2001, 451] admet que cette pratique engendre " dans le désordre de nouveaux spectacles individuels » tout en l'attribuant à des " lecteurs déculturés ». Christian Vandendorpe [2011, 55] propose, à l'instar de Michel Butor, de distinguer entre un « zapping 
passif » tendant vers le brouhaha, et un « zapping actif » luttant contre la pauvreté de l'information.

Comme Christian Vandendorpe, Claire Bélisle [Bélisle, 2011, 9] conseille de relativiser les craintes souvent exprimées quant aux conséquences cognitives et culturelles de la lecture rapide sur les supports numériques en les replaçant dans le contexte de l'histoire de la lecture. L'accès à l'information s'est accéléré progressivement de l'Antiquité à nos jours, d'abord grâce à l'émergence de l'espacement entre les mots, du découpage du texte en paragraphes, de la ponctuation, au passage de la lecture à voix haute à la lecture silencieuse, à l'imprimerie rendant le texte accessible à un nombre grandissant de lecteurs... Toutes ces évolutions ont provoqué de fortes réticences chez ceux qui non seulement devaient s'habituer à de nouveaux modes de présentation du texte, mais qui sentaient s'évanouir leurs privilèges. La « fureur de lire » notamment, qui se serait emparée de certains pays européens telle l'Allemagne au cours du $\mathrm{XvIII}^{\mathrm{e}}$ siècle en valorisant la variété, la quantité et la rapidité des lectures, a été critiquée par certains contemporains comme une « course effrénée » [voir Wittmann, 2001, 355], tandis que d'autres la considéraient déjà comme un acte de libération. "L'intensité avec laquelle se pratiquait la lecture réflexive critique des humanistes en faisait une lecture d'élite, marquée par l'autorité de ceux qui la pratiquaient », commente Claire Bélisle [2011, 17], et propose d'interpréter la fureur de lire au xvIII siècle comme une quête d'autonomie.

Selon Petrucci [2001, 449], le lecteur " postmoderne » clame de même « je lis ce que je veux » face à « la crise des structures institutionnelles et idéologiques qui avaient jusqu'ici soutenu l'ancien ordre de la lecture ». Hans Magnus Enzensberger [1983, 20] affirme que la lecture est un acte " anarchique », et revendique une absolue liberté du lecteur à l'encontre de la tradition autoritaire " critico-interprétative ». Pierre Bourdieu [2003, 295] fait remarquer que « lorsque le livre est un pouvoir, le pouvoir sur le livre est évidemment un pouvoir », prônant l'idée qu'un livre peut aussi agir « à travers ses contresens ».

La lecture rapide du texte encourt donc certes le risque du « contresens » en ignorant les " signaux faibles » [voir Boullier, 2011, 51]. Cela ne veut pas dire que le lecteur n'en retient rien : " Passer de lien en lien peut devenir ainsi, non pas seulement un parcours, mais la production d'un corpus, qui peut lui-même devenir un document », commente Dominique Boullier [2011, 53]. La pratique rapide ne transforme pas obligatoirement le lecteur en victime des « industries de l'attention ». Le problème est plutôt que la lecture rapide sur support numérique se trouve souvent associée 
à une " culture de la connaissance explicite ", définie par Claire Bélisle [2011, 145] comme « tous ces aspects de la connaissance qui peuvent être formalisés par des algorithmes ».

Tout texte peut être lu de façon rapide. Lorsque la lecture rapide s'impose pourtant non pas comme pratique occasionnelle, mais comme seule possibilité de réception du texte numérique ; lorsque la recherche d'informations devient le seul modèle de référence, les textes numériques eux-mêmes risquent d'être écrits et présentés de façon à favoriser cette pratique, au détriment des fils discursifs plus complexes de l'argumentation ou de la narration. Lors d'une étude quantitative et qualitative menée auprès d'adolescents sur la lecture de la presse, Barbier-Bouvet [2011, 130] a constaté que certains lecteurs reprochent par exemple à Télérama de proposer un discours trop « dialectique ». Ils préfèrent l'expérience à l'expertise, survalorisent les « faits » par rapport à l'argumentation : « les jeunes lecteurs n'aiment pas les textes qui les laissent sur un questionnement » et cherchent une "utilité » immédiate de la lecture : recherche qui peut rappeler les pratiques scholastiques du passé.

À l'encontre de la méthode monastique axée sur la compréhension lente et la méditation, la scolastique avait en effet déjà favorisé la composition de florilèges, qui réduisaient le texte original à un enchaînement de citations décontextualisées, faciles à parcourir. Les textes n'étaient plus traités comme des œuvres conçues à une époque précise, mais comme des corpus impersonnels de propositions [Hamesse, 2001, 136]. En réaction aux dérives de ce modèle, les humanistes de la Renaissance recommandaient de reprendre un contact plus direct avec les textes originaux. Comme l'explique Grafton [2001, 245], l'élève humaniste était amené à traiter tout texte comme une " chambre d'écho » en repérant notamment les allusions à des intertextes.

Le « jeu de clefs » pour réussir cette démarche était détenu par le maître humaniste. Voilà pourquoi un soupçon d'élitisme a toujours plané sur l'humanisme. Montaigne affirmant « feuilleter » ses livres « sans ordre et dessein ", " à pièces décousues ", doit régulièrement servir de modèle pour prouver comment le schéma de la lecture humaniste peut être brisé avec « liberté et brio » [voir Grafton, 2001, 256].

À l'heure actuelle, les discours revendiquant l'émergence d'un « humanisme numérique » se multiplient [voir notamment Doueihi, 2011 et ses multiples interventions dans des conférences], et la lecture y tient, à mon avis, un rôle important. La lecture de survol du texte numérique semble certes répondre à des motivations spécifiques, dont celle de localiser 
rapidement une information. Fondée sur la traversée rapide d'un grand nombre de textes, elle peut, comme l'affirment les auteurs travaillant sur la sérendipité [par exemple, Perriault, 2000 ; Ertzscheid, Gallezot, Boutin, 2009], permettre des découvertes insoupçonnées. Elle peut pourtant aussi fragiliser le lecteur, le rendre plus vulnérable aux tentatives de manipulation [voir Desrichard, 2011] - surtout si elle reste la seule pratique du texte numérique.

\section{Diversification des figurations}

Dans une étude menée par Yves Jeanneret, Annette Béguin, Dominique Cotte et al. en 2003 (154), certains sujets interrogés ont affirmé que la lecture de survol ne constitue qu'une première étape de découverte du texte numérique. Ils se promettaient de lire une sélection de textes «plus tard », quand ils auraient le temps. Si l'omniprésence d'un sentiment de pression temporelle a bien été constatée, certains lecteurs rêvaient de toute évidence d'un autre temps de lecture, effectuée " à tête reposée ", permettant de suivre des argumentations complexes [Jeanneret, Béguin, Cotte et al., 2003, 123].

Dans l'étude menée en 2006 à l'université Lyon 2, plus de $50 \%$ des étudiants interrogés ont affirmé imprimer le texte numérique pour entreprendre cette phase de lecture lente et concentrée. Alors qu'un premier groupe d'étudiants a indiqué que l'on « comprend moins bien » et que l'on « apprend mal » sur support numérique, que le texte électronique n’est pas propice à la révision et à l'apprentissage, d'autres sujets ont cependant considéré la lecture sur écran comme « rapide, intéressante, claire, accessible », " facile », " pratique », " intuitive et immersive », " moins statique et plus moderne ». Un étudiant est allé jusqu’à affirmer que « c'est devenu une habitude ; j'ai même du mal à lire en format papier ».

Lors d'une étude menée courant 2010 à l'université de Californie [Li, Poe, Potter et al., 2011], 49 \% des étudiants interrogés ont encore affirmé préférer les livres papier. De premiers basculements s'observent cependant, et laissent penser que de futures générations recourront au texte numérique non plus seulement pour la recherche d'informations, mais pour la lecture-plaisir et la lecture-apprentissage. Au cours d'une étude dirigée par Bowker [2012] auprès de jeunes étudiants britanniques en décembre 2011, deux tiers des sujets ont certes dit préférer les ressources papier pour lire dans le cadre de leurs études. La comparaison avec une étude semblable en 2003 montre néanmoins un changement significatif. 
Alors qu'en 2003, $95 \%$ des étudiants ont affirmé utiliser des ressources papier parallèlement aux ressources numériques, ils n'étaient plus que $88 \%$ en 2011. $49 \%$ ont déclaré un usage fréquent de Wikipédia. Constat d'un changement des pratiques qui s'était annoncé dès 2006 dans une étude par questionnaire menée par l'Observatoire national de la vie étudiante en France [2006], dans laquelle 9 étudiants sur 10 déclaraient utiliser Internet pour leurs études.

Bien évidemment, il ne s'agit pas ici d'affirmer que tous ces résultats sont strictement comparables, ces sondages ayant été effectués dans des contextes et avec des protocoles différents. Il est néanmoins possible d'y déceler des tendances. L'étude menée par l'éditeur Pearson [2012] aux États-Unis auprès de 1410 étudiants confirme pour la première fois une tendance qui s'annonce depuis plusieurs années : 6 étudiants sur 10 ont affirmé préférer la version numérique à la version imprimée d'un livre, qu'ils le lisent pour la détente ou pour les études. Le basculement vers la version numérique a eu lieu entre 2011 et 2012 : lors d'une étude semblable réalisée par le même éditeur l'année précédente, les déclarations en faveur du livre imprimé avaient encore été majoritaires.

$\mathrm{Au}$ vu de ces résultats, il paraît donc probable que beaucoup de lectures s'effectueront dans les années qui viennent sur les dispositifs numériques, que ce soit dans un cadre pédagogique, professionnel ou privé, qu'elles aient comme motivation la recherche d'informations, l'apprentissage, la juxtaposition de points de vue ou l'immersion dans des mondes fictionnels. Une diversification des figurations de la lecture numérique se dessine, incluant la possibilité de pratiques intensives. L'évolution des figurations du dispositif et du réseau y contribue certainement.

\section{FIGURATIONS DU DISPOSITIF ET DU RÉSEAU}

Afin de circonscrire l'évolution de l'horizon d'attente du lecteur face au texte numérique, il est instructif d'étudier le vocabulaire utilisé qui laisse parfois transparaître des prises de position fortes. Un premier champ sémantique s'organise autour de termes empruntés au milieu aquatique. Comme l'écrit Bertrand Gervais [2004, 61], « on navigue sur une mer, c'est-à-dire qu'on ne fait que rester en surface d'un lieu qui possède pourtant une densité et une profondeur, même si elles sont différentes de celles de la terre ferme ". À partir du moment où l'acceptation d'une métaphore comme celle de la "navigation » sur Internet est 
si communément partagée, il faut s'interroger jusqu'à quel point celle-ci décrit une pratique, ou participe à instaurer et légitimer cette pratique. Dans le cas du "surf », des idées de parcours de surface peuvent se coupler à des représentations de l'Internet comme espace difficilement maîtrisable, dans lequel le lecteur se laisserait emporter par toutes sortes de vents et courants ; la navigation peut pourtant aussi renvoyer à des idées de domination possible.

Je reviendrai sur ces questions de vocabulaire un peu plus loin en me penchant sur le terme "information ", si fréquent dans les discours consacrés à Internet : il suffit de penser à la récurrence des termes « autoroutes de l'information » et "société de l'information » pour comprendre à quel point ils ont forgé les imaginaires de l'Internet. Associés à des idées de transparence et d'universalité [Doueihi, 2011, 56], ces termes renvoient souvent à l'espoir que l'information numérique sera capable de réduire nos incertitudes sur le monde. Ne dit-on pas très couramment : " Je vais vérifier sur Internet » ? D'une part émerge dans ce discours l'idée qu'Internet constitue un lieu stable et fiable pour les « archives de l'humanité » [Doueihi, 2011, 56] et saura fournir une réponse à tout. D'autre part, des expressions comme " flux d'information » couplent des idées d'instabilité et d'éphémère, d'instantanéité et d'évanescence à ces idées de stabilité et de vérité, et révèlent un champ de tensions.

\section{Intelligences collectives}

À travers la figuration d'un Internet comme "mémoire » infiniment extensible et indéfiniment associative, le dispositif numérique est plutôt investi de l'idée de stabiliser les connaissances. Un " mythe fondateur » de l'Internet, comme le formule Milad Doueihi [2011, 56], consiste à prôner que tout sera désormais rendu accessible, que le " réseau des réseaux » accueille les " archives de l'humanité » tout en faisant émerger une « intelligence collective et interconnectée » [Lévy, 1997, 178]. À travers le concept d'" intelligence collective », le fonctionnement techno-sémiotique de l'hypertexte a été rapproché du fonctionnement complexe du cerveau humain. La nature " associative » des relations entre les " nœuds » d'un " réseau » hypertextuel a été considérée comme un premier pas vers « une relation directe entre le cerveau humain et les ordinateurs, condition déterminante de la symbiose planétaire par l'intermédiaire des réseaux de communication » [de Rosnay, 1995, 103 ; Lévy, 1997]. 
"L'informatique permet ici d'instrumentaliser le fonctionnement même de la pensée et de la construction des savoirs ", a affirmé aussi Jean Clément [2000, 49], mobilisant la figuration d'un Internet hypertextuel qui contiendrait non seulement les archives de l'humanité, mais toutes les lectures possibles de celles-ci. Jay David Bolter [1991] a avancé l'idée que l'« espace » d'écriture électronique reflète la façon dont fonctionne l'esprit en rendant visibles les connexions entre idées. "L'hypertexte reconduit la pensée à une organisation en réseau plus proche de la capacité associative qui caractérise l'organisation de la pensée », a écrit également Alessandro Zinna [2002].

Ces prises de position assez répandues dans les années 1990 reflètent la fascination des sciences humaines pour le champ de l'intelligence artificielle. Elles s'appuient en outre sur la complexité visuelle des graphes avec lesquels on essayait dans ces années de cartographier à la fois l'Internet dans son ensemble, et les relations entre les pages d'un site Web reliées par liens. Des graphes comme ceux réalisés dans le projet « Opte $»^{3}$ rappellent en effet, jusque dans les choix graphiques, les représentations des réseaux neuronaux.

Milad Doueihi [2011, 152] montre comment cette figuration d'un dispositif numérique saisissant l'intelligence, la mémoire et la vie se traduit dans le projet « My Life Bits », qui promet à l'usager de pouvoir stocker une " vie humaine » entière sur support numérique et de rendre cette vie explorable à travers des annotations et des hyperliens. Les auteurs de ce projet se positionnent à l'encontre des associations entre le Web « liquide » et les idées de l'éphémère numérique, affirmant que la mémoire numérique aurait un avantage immense sur la mémoire humaine : celui de ne pas connaître des failles de stockage.

Certaines créations de littérature et d'art numériques font appel à cette figuration d'une mémoire humaine à la fois stabilisée et explorable (voir l'hyperfiction Inside - A Journal of Dreams ${ }^{5}$ évoquée plus haut), alors que d'autres mettent au défi ces attentes en explorant la labilité du dispositif numérique [Gregory Chatonsky dans ses œuvres « flux $»^{6}$; mes créations "Flux » et « Tramway »; pour une contextualisation théorique de ces créations, voir Saemmer, 2011b et Gervais, 2011].

3. «The Opte Project », [en ligne] : < http://opte.org/ >.

4. Projet « My Life Bits », [en ligne] : < http://research.microsoft.com/en-us/projects/mylifebits/ >.

5. Andy Campbell, Inside - A Journal of Dreams, [en ligne] : < http://www.dreamingmethods.com/ inside/ >.

6. Voir par exemple Gregory Chatonsky, La révolution à New York a eu lieu, [en ligne] : < http:// www.incident.net $>$. 


\section{Exploration d'espaces}

La représentation arborescente des parcours est également à l'origine d'une figuration de l'hypertexte comme espace de parcours de lecture possibles, comme le font remarquer Jean Davallon et Yves Jeanneret [2004, 47]. Quand George P. Landow [1997, 36] avance que l'hypertexte lie un bloc textuel à un ou plusieurs autres sans établir une hiérarchie d'exploration précise, il considère en effet l'hyperlien comme une partie d'un espace explorable.

Dans les publications académiques consacrées à l'hypertexte, les graphes se sont multipliés tout au long des années 1990 et 2000. Mark Bernstein [2003] a proposé des représentations comme le "circle» (un hypertexte qui ramène le lecteur vers des textes déjà visités) ou le " contour » (des cercles mutuellement interconnectés), Marie-Laure Ryan [2006, 148] a parlé d' " anémone de mer » ou de "labyrinthe ». Tout en reflétant le besoin de repères, ces figurations ne contenaient généralement aucune indication sur les raisons qui ont amené un auteur à relier des textes par des hyperliens. Elles opéraient donc « une sélection importante dans ce qui peut être pris en compte par l'analyse ", comme le formulent Jean Davallon et Yves Jeanneret [2004, 47] : le potentiel d'action du texte numérique a été réduit à une " combinatoire graphique de parcours », orientant les attentes du lecteur vers l'idée qu'il importe plus d'explorer comment différents textes se trouvent reliés, que d'interpréter pourquoi ils le sont.

\section{Partages universels}

Les figurations permettent "à une société de construire son identité, en exprimant ses attentes par rapport au futur »[Flichy, 2001, 254]. Patrice Flichy a montré leur importance dans de nombreuses publications. Dans son livre L'imaginaire d'Internet [2001], il met en avant le rôle de la contreculture californienne dans l'émergence d'un « imaginaire coopératif »du Web - figuration d'autant plus prégnante qu'elle s'appliquait aussi bien à la technique qu'à l'organisation sociale du réseau.

Le principe de coopération et de partage a constitué en effet, selon les affirmations de certains fondateurs du Web, à la fois la raison d'être de l'architecture technique du World Wide Web en tant que réseau d'ordinateurs " décentralisé », et le principe de fonctionnement "démocratique » de sa mise en place. Dave D. Clark, coordonnateur de l'Internet Engineering Task Force (IETF) de 1981 à 1989, a résumé la philosophie du 
groupe qui a produit certains standards du Web : "We reject kings, presidents and voting. We believe in rough consensus and running code"7. L'IETF se voulait informel, international, ouvert à tout individu souhaitant présenter ses idées - sans prise en compte de sa profession, de ses diplômes et autres qualifications qui avaient garanti l'accès à la prise de parole dans l'espace public traditionnel.

Dominique Cardon [2010] a analysé la récupération des technologies du réseau au sein des mouvements autonomistes américains, qui investissaient rapidement le Web émergeant dans l'idée d'élaborer des modes de vie plus ouverts et démocratiques. Significativement, le groupe travaillant sur les protocoles de communication du réseau (Network Working Group) intitulait ses comptes rendus de réunions Requests for Comments : toute proposition était soumise au débat public, dans l'espoir qu'émerge une « intelligence collective » de cette foisonnante communauté où les idées se juxtaposaient, se confrontaient, se discutaient et se développaient collectivement : l'intelligence étant plus que la somme des propositions avancées par chaque individu isolé. "Ils rêvent d'un monde [...] où les communautés en ligne renforceraient ou remplaceraient les communautés locales, où les conférences informatiques permettraient d'exercer une intelligence collective, de construire un nouvel espace public », résume Patrice Flichy [2003]. Comment une idée mise au point par un petit groupe d'ingénieurs a-t-elle pu participer à forger une figuration socialement partagée de l'Internet?

Pour appréhender ce processus, il faut considérer le fait que des pionniers comme Howard Rheingold [2000] ont présenté dans les années 1990 ces expériences communautaires comme modèle de référence. Bien que les enjeux politiques et commerciaux de l'Internet aient été rapidement exploités par les élites qui avaient déjà dominé l'espace public traditionnel, et que le financement de la mise en place de l'Internet à l'échelle mondiale n'ait jamais été indépendant, le Web a été présenté dans les médias de l'époque comme le reflet d'une contre-culture. En 1993, le Time Magazine a intitulé " Cyberpunk » le dossier de couverture de l'un de ses numéros, qui a salué Howard Rheingold comme incarnation du « premier citoyen d'Internet $»^{8}$. En 1995, alors que Windows lançait la version 1995 de son système d'exploitation incluant des technologies clés pour la connexion à Internet, et que le navigateur Internet Explorer se trouvait désormais préinstallé sur les ordinateurs dotés de ce système, Time a

7. Internet Engineering Task Force, [en ligne] : < http://www.ietf.org/tao.html >.

8. Time Magazine, 8 février 1993, [en ligne] : < http://www.time.com/time/covers/0,16641,19930208,00. html >. 
prôné l'idée d’un réseau « non propriétaire », non contrôlé, fonctionnant comme une commune avec 4,8 millions de membres.

Une étude menée en 2010 sous la direction d'Aurélie Laborde à partir de l'analyse de 1642 articles du Figaro et du Monde révèle que les figurations de l'Internet véhiculées par la presse française s'approchaient de ces représentations relayées par les médias américains. Jusqu'au début des années 2000 s'affichait en effet une vision assez optimiste et confiante de l'Internet, mettant au centre l'idée de liberté couplée au mythe du far west. Dirigées contre la récupération commerciale des valeurs de partage et de bénévolat, ces idées continuent aujourd'hui à animer les discours autour des logiciels open source, et servent en outre à légitimer certaines formes de piratage. Pour Faucilhon [2010], les pirates sont ainsi les «nouveaux promoteurs de la libre circulation des idées, les derniers défenseurs de l'utopie de partage » [Tarin, 2011, http://bbf.enssib.fr/consulter/ bbf-2011-03-0104-003].

Dans la lecture sociale nourrie de commentaires parfois divergents, cette figuration est actuellement censée trouver son apothéose. Si les connotations négatives de la navigation associent le dispositif numérique, et en particulier l'Internet, à des lectures rapides et superficielles, à une « tyrannie de l'immédiat et de l'instantané » [Doueihi, 2011, 11] qui contaminent la réflexivité, ses connotations positives renvoient à des idées de transparence et de partage universel, qui, avec les outils du Web 2.0, s'associent de plus en plus à l'idée d'une lecture enrichie. Si les uns déplorent la disparition de la lecture au profit d'une " communication exubérante et au droit d'être auteur désormais reconnu à chacun » [Finkielkraut, Soriano, 2001, 31], d'autres présentent la lecture sociale comme un modèle résolument démocratique. Certains auteurs, tel François Bon sur son site tierslivre.net, ont rapidement investi l'hyperlien d'une fonction sociale qui semble, dans les pages plus récentes du site, absorbée et redéployée dans les commentaires et une activité d'écriture sur les réseaux sociaux. L'idée de partage, de transparence et de droit d'expression accordé à tous reste donc l'une des figurations puissantes de l'Internet.

\section{Connaissances en réseau comme processus}

Malgré les précautions à prendre quant aux « présuppositions d'égalité » inhérentes à l'idée du partage universel (le partage sur Facebook est par exemple fortement conditionné par la notion d'« amis »), Dominique Cardon [2009] défend l’idée que l'espace public s'est élargi grâce au Web : 
Internet « a désenclavé l'espace fermement contrôlé et contenu de l'expression publique en l'ouvrant à de nouveaux énonciateurs ", donnant l'impression que la parole de tous ceux qui avaient auparavant dominé l'espace public - journalistes, hommes politiques et experts -, pouvait enfin être efficacement contestée. L'encyclopédie participative Wikipédia constitue selon Cardon une " figure exemplaire » de cette ouverture : contrairement aux encyclopédies comme Universalis et Britannica rédigées par des experts, les contributeurs de Wikipédia seraient évalués par la communauté des rédacteurs à partir de ce qu'ils écrivent, et non pas à partir de leurs diplômes ou autres qualifications socialement reconnues.

Se pose néanmoins la question des figurations dont les lecteurs investissent ce projet explicitement présenté comme une encyclopédie - autrement dit, si les attentes envers l'encyclopédie ont évolué aussi rapidement que ses modes d'écriture et de publication ${ }^{9}$. Comme le montre Dominique Cardon, l'espace public traditionnel est le résultat d'un long processus de " professionnalisation » et de "domestication » des locuteurs. Ceux qui prennent la parole dans l'espace public traditionnel le font grâce à des statuts spécifiques, fondés entre autres sur l'organisation syndicale de la presse et de l'édition et sur des outils de protection de la liberté d'expression. Malgré ces aspects sans doute injustement exclusifs, l'information se trouvait alors sélectionnée au préalable par des professionnels, selon une certaine déontologie et suivant une " contrainte de distanciation » censées mettre à l'écart des formes de prise de parole trop subjectives ou intéressées [voir aussi Gaschke, 2009]. L'énonciation éditoriale des sites comme Wikipédia et de certains journaux en ligne peut susciter des attentes de pérennité, de fiabilité et d'expertise indépendante associées aux figurations du dispositif de lecture papier - attentes potentiellement mises au défi par le fonctionnement actuel de ces sites.

La figuration de l'Internet comme espace d'émergence d'une intelligence collective, addition de subjectivités et de savoirs forcément relatifs, nécessite en effet que la connaissance soit perçue comme un processus admettant la mise en doute, et non pas comme un produit stable, transparent et vrai. Loin de l'idée que l'on vérifie des informations sur Internet, la représentation du Web comme espace public ouvert, fondé sur une " libération des subjectivités " [Cardon, 2009] et un droit de parole donné à tous, de l'Internet décentralisé dans lequel les opinions se croisent, se juxtaposent et se contredisent, semble donc impliquer de la part du lecteur

9. Voir projet « Encyclopédies en ligne et lectures numériques » (ELLEN), décrit à la note 19, p. 60. 
une acceptation, au moins transitoire, de l'incertitude. De toute évidence, certains lecteurs adhèrent à cette figuration et prônent ses avantages voir l'affirmation de Kevin Kelly [2010 ; éditeur du magazine Wired] qu'il est désormais moins intéressé dans la " vérité, avec un grand V, et plus intéressé dans les vérités, au pluriel ».

Cette affirmation fait penser au " dialogisme » tel qu'il a été défini par Mikhail Bakhtine [1929], donc à l'idée que la vérité n'est pas individuelle mais se construit grâce à l'interrelation dialogale : elle " naît entre les hommes qui la cherchent ensemble, dans le processus de leur communication dialogique » $[1970,155]$. Selon l'ancien président de Reuters New Media, Andrew Nibley [2000, 37-38], la figuration d'un tel Internet " dialogique » répond notamment aux attentes des jeunes lecteurs, qui ne veulent plus qu'on leur dise " comment penser ».

\section{Connaissances en réseau comme produit}

Or, des études empiriques montrent aussi que beaucoup de lecteurs attendent du texte numérique qu'il fournisse de l'« information » (voir l'étude de 2006, note 19, p. 60, et l'étude exploratoire à l'université Paris 8 décrite plus loin). Ils entendent par « information » des données «factuelles », des définitions, descriptions, dates et détails capables de réduire leur incertitude sur le monde. L'information devient dans cette figuration " une sorte de réel ontologique immédiatement perceptible » [Le Marec, Babou, 2003, 255] relevant d'une « culture de l'évidence ».

La présence du mot « information » dans les discours scientifiques et journalistiques circonscrivant les spécificités de l'Internet est frappante. Un amalgame entre "données ", " information ", " savoir ", " connaissance " et "vérité " s'opère dans certains discours au détriment des termes " opinion », " prise de position », « subjectivité » et « relativité » caractéristiques de la vision communautaire et dialogique de l'Internet.

L'étude des représentations de l'Internet dans la presse française [Laborde, 2010] montre ainsi que dans la période de 2004 à 2007, celles-ci renvoient avant tout à une figuration du Web comme "source de connaissance ". Cette source de connaissance s'envisage à cette période sous un angle unilatéral, de l'émetteur " expert » vers le récepteur en manque d'informations, et renvoie à une " culture de la connaissance explicite » [Bélisle, 2011, 27] : comme le montrent Joëlle Le Marec et Igor Babou [2003, 255], cette figuration est fondée sur l'idée que « la communication entre individus serait enfin désambiguïsée, libérée des contraintes de 
l'interprétation ». C'est précisément lorsqu'Internet est considéré comme dépositaire de cette sorte de connaissances que la phrase « je vais vérifier sur Internet » prend sens. Cette phrase ne reflète décidément pas l'idée d'une connaissance comme processus issu d'une " culture de l'argumentation ", mais comme produit issu d'une " culture de l'évidence ».

Dans ce contexte, il paraît nécessaire de revenir brièvement à l'institutionnalisation du terme "société de l'information ». Cette institutionnalisation a donné lieu depuis les années 1970 à de nombreuses annonces officielles et initiatives gouvernementales. Comme le montre Sarah Labelle [2001], l'annonce " participe matériellement de la réalisation même de ce qu'elle prétend désigner : ainsi passe-t-on d'un effet de discours, dans le dire, à l'autoréalisation d'une idéologie, dans le faire » (66). Une initiative datant de 1997 porte le titre significatif " Programme d'action gouvernemental pour la société de l'information » $(\mathrm{PAGSI})^{10}$, posant l'existence de cette "société de l'information " comme un allant-de-soi, et témoignant de la volonté du gouvernement d'y prendre une part active. Dans les publications officielles autour de ces initiatives et programmes, les termes "Internet » et « information » se trouvent étroitement liés. Ne dit-on pas en effet, très communément, et même dans les curricula universitaires, "recherche d'informations » et non pas "recherche d'opinions " ou " repérage de points de vue » pour qualifier la recherche sur Internet, et cela même si la "société de la connaissance » a désormais remplacé la « société de l'information » dans les discours officiels [Bélisle 2011, 22] ?

Certes, lorsque le Journal officiel de l'Union européenne liste en 2006 les « compétences clés pour l'éducation et la formation tout au long de la vie $"^{11}$, l'accent est non seulement mis sur la recherche, mais aussi sur l'évaluation de l'information. Celle-ci reste néanmoins investie d'une idée de légitimité, d’unité du «message » [Wolton, 2009, 55]. Celui qui dit « je vérifie sur Internet » considère les données disponibles sur le Web comme des « informations » qu'il investit de l'espoir d'être porteuses de certitudes.

L' illusion technologique " peut renforcer cette idée d'une " vérité » déposée sur Internet. Comme l'explique Hervé Laroche [1999], l'illusion technologique n'est pas spécifique aux " technologies de l'information ", mais y est particulièrement prégnante : "Elle consiste à croire que tout problème trouve une solution par un surcroît de technologie ». L'hyperlien

10. Le « Programme d'action gouvernemental pour la société de l'information », [en ligne] : < http:// www.ddm.gouv.fr/article.php3?id_article $=841$ >.

11. « Compétences clés pour l'éducation et la formation tout au long de la vie », [en ligne] : < http:// europa.eu/legislation_summaries/education_training_youth/lifelong_learning/c11090_fr.htm >. 
joue un rôle clé dans cette illusion technologique grâce à l'instantanéité avec laquelle il donne accès aux données.

\section{FIGURATIONS DE L'HYPERLIEN}

Le lecteur nourrit aussi des attentes envers le texte lui-même à lire, avec ses répertoires, procédés rhétoriques et formes-modèles. Peu de spécificités du texte numérique ont généré autant de prises de position divergentes que l'hyperlien.

\section{Non-séquentialité et systèmes associatifs}

Lorsque Theodor Nelson a créé en 1965 le néologisme " hypertexte », il rêvait d'abord d'un gigantesque projet de bibliothèque : "Xanadu » devait interconnecter la totalité des archives humaines. De nouvelles formes de liens «non séquentiels » entre les documents de cette archive devaient permettre une lecture des fichiers dans " toutes les directions " [voir Nelson, 1982]. Dès le départ, la «non-séquentialité » de l'hypertexte s'est trouvée opposée à la « séquentialité » de la page de livre, et cela même si tout livre n'anticipe évidemment pas sur une lecture séquentielle : les dictionnaires et encyclopédies, certains recueils de poésie et livres d'artistes, beaucoup de journaux et magazines ne se lisent pas du début jusqu'à la fin.

L'idée de la non-séquentialité a été largement promulguée par les premiers théoriciens de l'hypertexte aux États-Unis, dont George P. Landow et Jay David Bolter, et les premiers auteurs d'hyperfictions qui la couplaient souvent à un refus de la " clôture " du texte, une relativisation $\mathrm{du}$ statut d'auteur et une revalorisation du statut de lecteur. L'hypertexte est tombé chez ces théoriciens et créateurs sur un terrain fertile, préparé par la lecture des théories poststructuralistes françaises et de la littérature d'avant-garde. A émergé une position que certains ont appelée par la suite " théorie de la convergence » et qui a suscité beaucoup de critiques, surtout depuis les années 2000 [voir notamment Cusset, 2003]. Il ne s'agit pas d'évaluer ici la validité des figurations inhérentes à la théorie de la convergence, mais de circonscrire son influence potentielle sur les pratiques du texte numérique.

George P. Landow [1997] a considéré dans les années 1990 les approches du texte et de la lecture poststructuralistes non seulement comme points de départ, mais comme véritables prophéties de l'aventure 
hypertextuelle (4). "What is unnatural in print becomes natural in the electronic medium and will soon no longer need saying at all, because it can be shown", a avancé de même Jay David Bolter [1991, 143]. Comme le montre Samuel Archibald [2009, 48], le poststructuralisme constitue une transition entre la vision immanente du sens proposée par le structuralisme, et l'idée du sens " négocié » résultant d'une fusion des horizons du texte et du lecteur qui sera précisée par les théories de la réception. Roland Barthes a, dans ses travaux poststructuralistes, redéfini la notion de « texte » en avançant que tout texte n'existe que par l'acte de réception. Il était tentant de prôner des convergences entre cette revalorisation du lecteur et le potentiel d'action de l'hypertexte, avec lequel le lecteur semble pouvoir interagir beaucoup plus amplement que dans le texte papier.

Influencés par le poststructuralisme, certains auteurs ont donc projeté sur l'hypertexte des idées de non-séquentialité, de structure " rhizomatique » et de texte "scriptible ». George P. Landow [2006] propose le néologisme "wreader" mélangeant les termes de lecteur et d'auteur. L'idée est également chère à Pierre Lévy : "Depuis l'hypertexte, toute lecture est un acte d'écriture » [1998, 44].

L'assimilation entre intertexte et hypertexte semblait également s'imposer de façon naturelle à cette époque. Roland Barthes et Julia Kristeva ont considéré le texte comme un réseau de références à d'autres textes [Barthes, 1973a ; Kristeva, 1969] qui se trouve actualisé par le lecteur. Suivant Michel Riffaterre [1980, 4], l'intertextualité se définit comme la « perception par le lecteur de rapports entre une œuvre et d'autres, qui l'ont précédée ou suivie » (9). Pour George P. Landow [1997, 4], l'hypertexte permet de visualiser de façon efficace ces relations intertextuelles. Ce qui, lors de l'acte de lecture, reste forcément intuitif et imprécis se manifesterait grâce à l'hypertexte comme une « référence », « facile à suivre » (c'est moi qui traduis).

L'intertextualité selon la définition poststructuraliste est pourtant infinie : il s'agit d'un phénomène de lecture. Je rappelle dans ce contexte l'observation avancée par Bertrand Gervais [2006] que l'hyperlien se comporterait comme un signe en renvoyant à quelque chose d'autre pour quelqu'un, sauf qu'il le ferait toujours de façon identique une fois programmé. La stabilité immuable du lien suggérerait l'élimination de l'interprétant : le lien et le renvoi existeraient, sans jamais varier, indépendamment du lecteur qui choisit de l'activer. C'est précisément pourquoi l'hyperlien placerait le lecteur "dans une logique de la révélation, de l'apparition de vérités » (234). Bertrand Gervais résume dans cette proposition une figuration de l'hyperlien et de l'hypertexte qui se trouvait déjà esquissée dans les écrits 
de Theodor Nelson [1982]. L'esprit humain opère par associations, avance le pionnier de l'hypertexte ; mais la mémoire est « transitoire ». Theodor Nelson a combattu toute sa vie les déficits et défaillances de la mémoire humaine. L'hypertexte lui paraissait l'outil rêvé pour dupliquer les processus associatifs complexes qui s'opèrent lors de l'acte de lecture.

Samuel Archibald [2009, 57] souligne qu'une assimilation entre intertexte et hypertexte réduit pourtant les potentialités sémiotiques de l'intertextualité : « une lecture qui n'accepterait comme intertexte que les liens proposés par l'hypertexte serait forcément déficitaire et tout à fait conditionnée ». Il en est de même pour l'association plus générale entre hypertexte et processus de lecture : l'hypertexte ne peut que refléter des associations entre des textes qu'un auteur (ou éventuellement les auteurs ayant programmé des processus d'insertion automatisés) a considérées comme pertinentes. Il propose ces associations à la lecture. Le lecteur, en revanche, reste libre d'interpréter ces associations suivant son propre horizon d'attente.

À la fin des années 1990, de plus en plus d'auteurs ont insisté sur cette fonction « délimitante » de l'hypertexte en s'intéressant à ce phénomène textuel non plus en tant que structure, mais en tant que séquence textuelle manipulable liant un texte précis à un autre texte précis. Certes, un hypertexte donne toujours lieu à des actualisations variables lors la lecture par différents sujets ; en proposant la relation entre deux textes, l'Auteur d'un hyperlien oriente néanmoins ce processus de réception.

\section{Délimitations et contiguiités}

"Links have a delimiting rather than empowering function", avance ainsi Astrid Ensslin [2007, 31]. Au lieu d'ouvrir le texte à un réseau d'associations infinies, l'hyperlien apporterait plutôt une " délimitation ». Cette délimitation s'est trouvée progressivement associée à l'idée que l'hyperlien aurait comme première fonction de "définir ». Dans son lexique de termes techniques, le Journaldunet décrit l'hypertexte comme « possibilité de relier deux éléments textuels par un lien », et cite comme exemple phare " la définition d'un mot [...] reliée à son occurrence »12. "I will argue that hypertext links are defining in nature”, a avancé Scott Lloyd DeWitt [1999, 118].

Comme l'a montré Olivier Ertzscheid dans sa thèse de doctorat [2001, 219], beaucoup d'auteurs académiques ont proposé dans les années 1990

12. Le journal du net, [en ligne] : < http://www.journaldunet.com/encyclopedie/php/commun/imprimer_definition.php?f_id_definition=460 >. 
une distinction entre deux types de liens : les « liens organisationnels » (ou « liens structurels ») et les « liens sémantiques » (ou « liens de contenu »). Le lien organisationnel est défini comme un hyperlien qui appartient à la structure d'une page et qui se trouve répété sur plusieurs pages d'un site. Sont appelés ainsi les hyperliens dans un menu, dans un pied de page, dans un en-tête ou un logo. Le lien sémantique, lui, connecte " les parties d'un texte discutant de choses similaires ", indiquant au lecteur une page " dont le thème est en rapport avec celui de la page en cours » : " par exemple, dans une librairie en ligne, on pourra guider l'internaute vers des ouvrages traitant du même sujet que celui évoqué dans la page en cours ». L'hyperlien organisationnel « bibliothèque » figurant sur toutes les pages d'un site est pourtant à la fois organisationnel et sémantique : il annonce une page en relation logique avec la signification du mot « bibliothèque ». Dans le lien structurel comme dans le lien sémantique, l'idée que l'hyperlien pourrait aussi relier des points de vue divergents n'est pas envisagée.

Comme je l'ai déjà indiqué plus haut, Lev Manovich [2001, 228] a donc considéré la métonymie comme l'unique principe structurant de l'hypermédia, renvoyant au fonctionnement des bases de données : un mot hyperlié vaudrait pour le texte auquel il renvoie. Une telle figuration de l'hyperlien se réfère à une culture de la connaissance explicite et de l'évidence, et suscite l'attente que l'hyperlien relie un mot à sa définition, un objet à son contenu, une date à un événement historique, un nom d'auteur à sa bio- ou bibliographie, un crime à ses preuves.

\section{Instruments de complexité}

Loin de cette figuration d'un hyperlien délimitant, Pierre Lévy [1998, 42] a considéré l'hypertexte comme le pivot central d'une " intelligence collective ». En avançant qu'il « instrumentalise la complexité », Jean Clément [2000, 49] a également mobilisé l'idée d'un hypertexte qui oppose à l'organisation ordonnée des savoirs une " organisation en système, plus floue, mais plus ouverte et plus dynamique ». Avec l'hypertexte, la période de l'histoire des sciences, inaugurée par Descartes et Galilée qui avait placé l'approche scientifique sous le signe de la délimitation et de l'ordre, serait définitivement révolue. Au lieu de prôner l'existence d'une "vérité ", l'association dynamique de points de vue permettrait de refléter la complexité des phénomènes qui nous entourent. L'éventuelle divergence entre les points de vue contribuerait à l'émergence d'une nouvelle compréhension du monde. 
La figuration de l'hyperlien délimitant permettant d'accéder à des données factuelles, et celle de l'hyperlien « dialogique » juxtaposant des points de vue de façon complexe se côtoient de plus en plus fréquemment dans les discours académiques à partir du milieu des années 1990. Émerge progressivement l'idée que seule la figuration délimitante répond aux attentes du lecteur, tandis que la figuration dialogique constitue un écart par rapport aux normes d'attente.

\section{Présomption d'information ou attentes de dialogismes}

Dans les années 1990, Davida Charney [1994] a proposé de distinguer entre une figuration "romantique » et une vision " pragmatique » de l'hyperlien : alors que la vision romantique et ses concrétisations dans l'écrit numérique défient les attentes du lecteur, la vision pragmatique offre une mise en relation hypertextuelle satisfaisant les « attentes informationnelles ». L'auteur précise qu'il n'a pas eu recours à des méthodes empiriques pour sonder les attentes du lecteur face à l'hyperlien. Le point de vue de Charney a néanmoins été largement partagé.

Norton, Zimmerman et Lindeman [1999, 184] ont ainsi avancé que des développeurs " professionnels » comme Jakob Nielsen, qui procurent depuis de nombreuses années des conseils aux webdesigners, s'inscrivent dans une approche " pragmatique » de l'hyperlien, qui aurait comme but de « livrer de l'information » au lecteur. Des théoriciens « littéraires » de l'hyperlien, en revanche, défendent, selon l'auteur, une approche « rhizomatique ». Astrid Ensslin [2007, 127] a renchéri plus récemment en proposant de différencier entre un hyperlien « factuel » et un hyperlien « littéraire ». L'hyperlien "factuel » est présenté comme la forme " conventionnelle » $\mathrm{du}$ lien ("Links are conventionally used to illustrate connections between ideas") : les connexions entre texte géniteur et texte relié y sont « transparentes » et « prédictibles ». L’hyperlien « littéraire » subvertirait délibérément cette transparence et créerait « certains effets esthétiques ».

Ces affirmations reflètent non seulement deux figurations importantes de l'hyperlien, mais sont imprégnées d'une certaine représentation du texte littéraire. La littérature se trouve investie de l'idée de déconcerter, de surprendre, d'innover et de défier les automatismes de la perception quotidienne, alors que le discours usuel, ordinaire, habituel aurait tendance à confirmer les attentes du lecteur.

La mise au défi des attentes n'est pourtant pas réservée au domaine de la littérature, et l'hyperlien littéraire confirme parfois les attentes 
factuelles du lecteur. Plus importante que l'association d'une représentation de l'hyperlien à un genre textuel paraît donc la question des « standards » tels qu'ils sont proclamés dans ces discours. En effet, les auteurs des typologies citées ont unanimement considéré la fonction informationnelle de l'hyperlien comme une " convention ».

Susana Pajares Tosca [2000] a différencié entre des « liens efficaces », mis en œuvre sur des sites Web qui évitent des dénominations équivoques, proposent une distinction claire entre les rubriques et annoncent d'emblée les particularités du texte relié : les efforts de traitement par le lecteur seraient minimaux et les effets cognitifs maximaux. Les « liens suggestifs » mettent au défi les attentes du lecteur en privilégiant la découverte insolite au lieu de la « recherche efficace ». Christian Vandendorpe [1999] a inscrit sa typologie de l'hyperlien dans cette même bipolarité. Il a proposé de différencier entre des liens qui « complètent » et " enrichissent » le contenu de façon "efficace ", et des liens qui amènent le lecteur à un autre domaine et «favorisent une lecture fractionnée ».

Emily Golson [1999, 157] a reconnu beaucoup de qualités à ces hyperliens « efficaces » qu'elle a choisi d'appeler, plus sobrement, "intersecting links". Elle a pourtant également insisté sur leurs limites. Selon l'auteur, ces liens facilitent certes la compréhension en reliant un texte à des définitions, à de l'« information établie ", à une " conclusion logique » : ils " indiquent des points de départ bien marqués vers des destinations prévisibles », et mettent en œuvre un « flux d'information » qui passe par une structure binaire et hiérarchique. Le lecteur peut approcher ces liens avec une question simple; l'hyperlien lui offre le chemin le plus rapide vers une réponse claire. La prévisibilité de cette relation peut rassurer. En revanche, elle n'invite pas à l'adoption d'une attitude réflexive. Aucun " sens inédit » ne peut, selon Golson, résulter d'une telle lecture hypertextuelle.

Emily Golson $[1999,158]$ a donc valorisé plutôt les liens " suggestifs », pour lesquels elle a proposé le terme "interacting links". Ces liens inviteraient le lecteur à s'engager sur des " chemins inconnus » : à lui de construire une contiguïté possible à partir des éléments en apparence divergents. L'auteur a souligné que le manque de contiguïté entre texte géniteur et texte relié comporte le risque de " piéger le lecteur dans les tourbillons vertigineux de la contradiction ». Néanmoins, seuls les "interacting links" peuvent " rompre des schémas de pensée familiers ». Les associations " imprévisibles » établies par ce lien peuvent, selon l'auteur, être comparées à des " figures de style » : contrairement aux mises en relation « littérales », elles impliquent des actes de « médiation » par le lecteur. 


\section{La présomption d'information en expérience}

L'idée d'un hyperlien "dialogique » défini comme médiateur entre idées divergentes, et celle d'un hyperlien « informationnel » défini comme lien stable entre données factuelles, constituent des figurations récurrentes dans les discours académiques étudiés. Les auteurs des typologies précitées n'ont pas eu recours à des études menées auprès de lecteurs. Ils ont déduit leurs conclusions de l'analyse du texte numérique lui-même, et de leurs propres impressions. Plusieurs études quantitatives et qualitatives menées ces dernières années convergent cependant avec ces résultats : avant d'entreprendre la lecture d'un texte précis, beaucoup de lecteurs déclarent en effet approcher l'hyperlien avec l'attente de recevoir des réponses factuelles. Ils adhèrent à ce que l'on pourrait appeler une figuration « informationnelle » de l'hyperlien, s'inscrivant dans une « culture de l'évidence » potentiellement hostile à la valorisation de l'argumentation.

Dans l'étude par questionnaire menée en 2006 auprès de 600 étudiants (voir note 19, p. 60), l'Internet s'est ainsi trouvé associé à la recherche d'informations. L'association forte avec les pratiques de survol montre que l'information a été considérée par ces lecteurs comme une donnée factuelle, que l'on consulte. Une étudiante a affirmé que l'exploration de textes par le biais d'hyperliens ne relève pas de la lecture. Elle a précisé que lire sur écran signifie " parcourir des documents afin de trouver une information ". Dans une étude du CSA effectuée en 2011 auprès de 1005 adultes en France [CSA, 2011], 76 \% ont également affirmé « consulter » avant tout « de l'information » sur Internet. Les sujets ont insisté sur l'avantage de la " rapidité d'accès ». Encore une fois, les termes " rapidité » et « information » se sont donc trouvés étroitement associés.

Pour sonder les attentes d'un groupe de jeunes adultes vis-à-vis de l'hyperlien tout en les sensibilisant aux potentiels d'action du texte journalistique en ligne, j'ai mené en avril 2011 à l'université Paris 8 une étude exploratoire auprès de 16 étudiants inscrits en Master 2 "Gestion de l'information et du document » et 27 étudiants en Master 1 « Création et Édition numérique ». Cette expérience d'une durée de 3 heures a, en M2, fait partie intégrante d'un cours intitulé « Méthodologie de la recherche » comportant 21 heures au total. En M1, l'expérience a été intégrée à un séminaire-atelier à fois théorique et pratique de 36 heures consacré aux "Signes et figures du discours numérique ». Dans les deux groupes, l'expérience a été programmée en début de semestre, après une première séance d'introduction. Dans les deux formations, les étudiants avaient 
déjà acquis des connaissances théoriques et pratiques en sciences et technologies de l'information et de la communication.

De façon générale, les étudiants intégrant ces formations peuvent être considérés comme des «insérés » du numérique. Nombre d'entre eux abordent l'histoire des technologies et ses évolutions récentes avec beaucoup de curiosité, voire une certaine fascination. Tous les étudiants avaient déjà consulté des journaux en ligne. 30 \% se sont déclarés « lecteurs réguliers » (lisant les journaux en ligne au moins une fois par semaine). La moyenne d'âge des étudiants était de 24 ans. L'expérience comportait une partie écrite et une discussion enregistrée. Les résultats obtenus dans les deux groupes n'ayant pas différé de façon significative, je les présente simultanément.

J'ai d'abord demandé aux étudiants de mettre sur papier une définition de l'hyperlien. Ils disposaient d'une quinzaine de minutes pour répondre. Je précise que le sujet de l'hyperlien n'avait pas encore été abordé dans ces cours, mais que les étudiants en avaient évidemment entendu parler ailleurs dans la formation. Émerge majoritairement des réponses la présomption d'information avec laquelle les étudiants approchent le mot ou l'image hyperliés. Vingt-huit étudiants attendaient ainsi de l'hyperlien qu'il fournisse un " complément d'information ». Ils ont circonscrit cette fonction informationnelle en avançant que l'hyperlien généralement « explicite » (5), « enrichit » (4), « éclaircit » (3), « approfondit » (5), « définit » (4), « illustre » (7), « décrit »(1) les informations contenues dans un texte. Les étudiants ont inscrit leurs réponses dans une définition restreinte de l'information comme " donnée sérieuse », " définition », « explication », « complément factuel » renvoyant à l’idée de "vérité ». La fonction éventuellement commerciale ou publicitaire de l'hyperlien a seulement été évoquée par deux étudiants.

Cette attente globalement informationnelle s'est confirmée dans l'étape suivante de l'expérience. J'ai distribué aux étudiants un article imprimé de lexpress.fr portant sur l'affaire Galliano. Cette interview avec un juriste porte sur le licenciement du créateur de mode John Galliano suite à des propos racistes tenus dans un bar parisien, et plus précisément sur les justifications juridiques de cette procédure. Les hyperliens ont été signalés par un soulignement. Dans cette première étape de l'expérience, il a été évidemment impossible d'activer les hyperliens ; les étudiants se trouvaient face à une version papier du texte. Pour chacun des six hyperliens, les étudiants devaient lister leurs attentes concernant le texte relié. 
Voici le document tel qu'il leur a été présenté. L’article avait été séparé du contexte de la page-écran avec ses formes-modèles afin de ne pas détourner l'attention.

\section{Encadré 1. Affaire Galliano : Dior peut-il licencier Galliano pour des propos racistes?}

par lexpress.fr, publié le $1^{\text {er }}$ mars 2011 à 18:15, mis à jour à 18:50

Dior va licencier le créateur John Galliano, accusé de propos racistes. Décryptage avec $\mathrm{M}^{\mathrm{e}}$ Rocheblave, avocat en droit du travail.

Peut-on être licencié pour des propos ou des faits tenus en dehors du cadre de son travail comme c'est le cas ici?

D’une manière générale, un employeur ne peut pas tirer de motifs de licenciement de la vie privée de ses salariés. Mais dans le cas de l'affaire Galliano, les conséquences de ses propos dépassent clairement le cadre personnel. La jurisprudence prévoit que si certaines actions ou propos portent préjudice à l'image de l'entreprise, une procédure de licenciement peut-être engagée. Le fait qu'une égérie de la marque affirme publiquement son profond désaccord avec le créateur de Dior ou que cette affaire s'affiche en une de tous les médias est une des raisons suffisantes pour justifier le licenciement de John Galliano car ils font une mauvaise pub à l'entreprise. De même, Dior, qui possède une clientèle internationale et donc multiraciale, peut invoquer des craintes de baisse des ventes pour expliquer cette décision.
La direction de Dior a expliqué son geste par le caractère " particulièrement odieux » des propos du créateur. Quelles autres actions ou type de paroles privées sont répréhensibles dans la sphère professionnelle?

Il n'y a pas de liste de choses que le salarié a le droit ou non de faire. Le droit du travail prévoit qu'un employeur peut invoquer n'importe quel motif pour un licenciement. Dans ce cas-là, cela concernait des propos racistes, mais cela peut être une condamnation, un engagement politique, voire même un retrait de permis de conduire si cela risque de mettre l'entreprise en péril. Un juge apprécie ensuite a posteriori si ce motif est recevable ou non.

La maison de couture a licencié le créateur alors que la procédure juridique est encore en cours et que jusqu'à son jugement John Galliano est présumé innocent. Est-ce légal ?

Oui, car le motif de ce licenciement ne concerne par les propos de John Galliano mais les conséquences de ceux-ci. Dans ce cas-là, déterminer si la vidéo qui circule sur internet ou si les propos des personnes qui ont porté plainte sont véridiques ne sert à rien. Seules les conséquences de l'affaire sur l'image de l'entreprise sont importantes. Donc même s'il est reconnu innocent, rien n’obligera Dior à le réembaucher.

Le premier hyperlien est posé dans l'introduction de l'interview : « Dior va licencier le créateur John Galliano, accusé de propos racistes ». Les réponses fournies par les étudiants ont montré qu'ils attendaient majoritairement (20) de cet hyperlien qu'il rappelle les " faits », qu'il fournisse des explications (5). Certains (8) ont pensé pouvoir accéder à la source de 
cette information en cliquant : ils s'attendaient à retrouver la première dépêche, le premier article relatant le fait divers. D'autres (6) ont espéré obtenir des informations biographiques sur le créateur. Beaucoup d'étudiants (15) ont souhaité avoir accès à la vidéo tournée dans le bar, ou à d'autres " preuves » comme des photos. Ils s'attendaient généralement à ce que le texte relié effectue une légère analepse temporelle par rapport au texte géniteur en proposant un retour sur les " faits ", éventuellement enrichi d'un flux d'actualité. Une attente allant vers une dénonciation des " méfaits » de Galliano, donc une prise de position de la part du journaliste, a été exprimée par 5 étudiants. Un étudiant seulement a évoqué la possibilité d'un hyperlien pointant vers une «parodie » de la vidéo ou une caricature de Galliano. L'attente face à ce premier hyperlien s'est donc avérée être majoritairement informationnelle.

Le deuxième hyperlien, posé sur le nom et le titre de l'interviewé, " $\mathrm{M}^{\mathrm{e}}$ Rocheblave ", a créé le plus grand consensus chez les étudiants : 36 d'entre eux s'attendaient à obtenir des « informations » biographiques sur l'avocat. Six sujets ont souligné que cette information biographique peut jouer le rôle d'un argument d'autorité en prouvant la fiabilité de la personne citée.

Les hyperliens suivants sont posés sur les paroles de l'interviewé. " D’une manière générale, un employeur ne peut pas tirer de motifs de licenciement de la vie privée de ses salariés. Mais dans le cas de l'affaire Galliano, les conséquences de ses propos dépassent clairement le cadre personnel ", explique l'avocat. Cet hyperlien a suscité encore une fois des attentes d'informations avant tout factuelles. Les étudiants ont pensé majoritairement (21) que le texte relié fournit un « rappel des faits », par exemple sous forme de compilation d'articles ou de flux d'actualité. Huit étudiants s'attendaient à ce que le texte relié fournisse des " explications ». Quatre sujets s'attendaient à un enrichissement du texte géniteur par des " compléments d'information ». Sept autres ont exprimé encore le souhait d'accéder à une preuve ou à une source, par exemple la vidéo tournée dans le bar. Un étudiant seulement a évoqué la possibilité d'un déplacement de focus, s'attendant à un article traitant plus spécifiquement de l'impact médiatique de l'affaire.

Selon l'interviewé, c'est aussi " le fait qu'une égérie de la marque affirme publiquement son profond désaccord avec le créateur de Dior » qui confirme le caractère public de cette affaire. Beaucoup d'étudiants (21) s'attendaient à ce que ce quatrième hyperlien mène vers la source du propos rapporté, ou vers une citation contextualisée. Six étudiants ont déclaré 
s'attendre à une révélation du nom de l'égérie, et un étudiant a verbalisé le suspense éprouvé face à cet hyperlien : "À noter que c'est le lien sur lequel j’aurais le plus envie de cliquer, pour découvrir de qui il s'agit ». Six autres sujets s'attendaient à des exemples d'autres égéries en désaccord avec des représentants ou responsables d'une marque. Quatre étudiants ont évoqué la possibilité que l'hyperlien dialectise le propos souligné en donnant la parole à John Galliano, juxtaposant ainsi les points de vue du juriste, de l'égérie et de l'accusé. Un étudiant s'attendait à ce que ce « profond désaccord » soit « argumenté » par le journaliste.

Par la suite, l'avocat interviewé dresse une liste d'actions pouvant provoquer un licenciement ; il cite entre autres l'exemple des « propos racistes ". Les étudiants s'attendaient majoritairement (28) à ce que cet hyperlien fournisse une définition du terme " propos raciste ». Wikipédia ou Jurispédia ont été cités comme références pour la définition des termes, ce qui montre à quel point ces supports sont investis d'une figuration informationnelle (dans le sens de "vérité » et de " fiabilité »). Certains étudiants ont pris en compte le contexte de l'hyperlien en l'interprétant comme une annonce du texte à venir (7) : ils ont affirmé s'attendre à ce que l'hyperlien pointe vers la source « vidéo » des propos racistes proférés par Galliano. Un étudiant a évoqué la possibilité d'un hyperlien dialogique, avançant que celui-ci pourrait mener vers le site Web du Front national pour dénoncer certaines prises de position de ce parti.

Le dernier hyperlien est posé sur une affirmation du journaliste : « La maison de couture a licencié le créateur alors que la procédure juridique est encore en cours et que jusqu'à son jugement John Galliano est présumé innocent. » Trente et un étudiants escomptaient une "définition » du terme juridique. Huit ont affirmé s'attendre à découvrir des arguments plaidant pour l'innocence de Galliano. Trois étudiants ont pensé que le terme pourrait être " discuté » en relation avec cette affaire. Un étudiant a évoqué l'association possible avec l'émission télé Présumé innocent : Galliano a été considéré comme un futur invité de cette émission.

Une fois la liste des attentes recueillies (cette phase a duré 35 minutes), j'ai révélé les textes reliés pour chaque hyperlien. Nous avons confronté les figurations de l'hyperlien suggérées par les étudiants aux figures de la lecture anticipées par les relations logiques entre le texte géniteur et les textes reliés. Dans cet objectif, j’ai projeté à partir de chaque hyperlien quelques paragraphes du texte relié et proposé un résumé.

Le texte relié au premier hyperlien " John Galliano, accusé de propos racistes " revient sur les événements en fournissant les raisons de 
l'accusation de Galliano, et répond donc aux attentes informationnelles de la plupart des étudiants - même s'il ne fournit pas les preuves matérielles du fait divers (par exemple la vidéo).

Le texte relié au deuxième hyperlien sur $\mathrm{M}^{\mathrm{e}}$ Rocheblave présente en effet la biographie de l'avocat, ses fonctions et compétences, et confirme donc pleinement les figurations informationnelles énoncées par les étudiants.

L'hyperlien sur " affaire Galliano " mène vers le même article déjà convoqué par le premier hyperlien, qui donne les explications de l'affaire tout en rappelant les faits. Encore une fois, on peut constater une correspondance forte entre les figurations et la figure de la lecture anticipée par cet hyperlien précis. Lors de la discussion qui a suivi la révélation des textes reliés, certains étudiants ont néanmoins exprimé leur déception, voire leur agacement devant le fait que le même article se trouve convoqué par deux hyperliens : les techniques de référencement faisant visiblement partie de leur horizon d'attente, ils accusaient le journal d'opportunisme.

L'hyperlien sur " une égérie de la marque affirme publiquement son profond désaccord » mène vers un texte relié qui donne les raisons de ce profond désaccord, et révèle le nom de l'égérie : Natalie Portman. Le texte relié satisfait donc à la fois la curiosité exprimée par certains étudiants de connaître l'identité de l'égérie, et explicite le désaccord en citant ses propos.

Le texte relié au cinquième hyperlien posé sur " propos racistes » revient brièvement sur les propos tenus, mais montre par la suite que John Galliano avait élu le métissage comme philosophie de son travail créatif. Alors que l'avocat n'évoque que des principes purement juridiques, le texte relié prend ici partiellement position pour Galliano. Les attentes des étudiants divergeaient fortement de ce potentiel d'action dialogique de l'hyperlien.

Pour le dernier hyperlien posé sur " présumé innocent ", la majorité des étudiants s'attendaient à une définition du terme. Huit d'entre eux avaient néanmoins envisagé de trouver des prises de position fortes dans le texte relié, et 3 étudiants s'attendaient même à trouver une juxtaposition d'arguments et de contre-arguments. En effet, le texte relié mène vers un autre article de lexpress.fr, dans lequel les milieux de la mode évoquent le manque de témoignages dans cette affaire, l'ouverture d'esprit générale de John Galliano et leur étonnement devant les propos tenus.

Le fait que certains textes reliés proposent donc un plaidoyer au moins partiel en faveur de John Galliano a surpris beaucoup d'étudiants. Lors de la discussion, ils ont exprimé leur étonnement devant la divergence entre les arguments purement juridiques de l'avocat qui justifie le licenciement, et les textes reliés apportant des points de vue personnels et amicaux en 
faveur du créateur. Plusieurs étudiants ont souligné le fait qu’une sorte de « débat » se crée ainsi entre le texte géniteur et les textes reliés.

Aucun étudiant ne s'était exprimé par écrit sur le fait que l'hypertextualisation d'une interview peut poser problème, dans la mesure où l'interviewé n'a sans doute pas pu influencer l'insertion de bifurcations dans ses propos. Dès que j'avais, lors de la discussion, attiré l'attention sur le genre textuel de l'article, 5 étudiants se sont déclarés " surpris », voire « choqués » par cette pratique.

En résumé, cette expérience a montré que certaines figures de la lecture anticipées par l'hyperlien, et notamment celle où le texte géniteur et le texte relié font dialoguer des points de vue divergents, ne rencontrent pas toujours les attentes des lecteurs vis-à-vis du texte numérique. La plupart des étudiants s'attendaient en effet à des données factuelles, des preuves, définitions, précisions ou compléments d'information, inscrivant l'hyperlien dans une culture de l'évidence.

Le répertoire du texte géniteur a néanmoins participé à l'orientation de leurs attentes. Pour l'hyperlien sur l'expression " présumé innocent » qui porte en elle-même un potentiel d'action dialogique, plusieurs étudiants pensaient découvrir un texte caractérisé par des prises de position fortes ; certains envisageaient même la possibilité d'une présentation d'arguments s'opposant au point de vue énoncé dans le texte géniteur.

\section{FIGURATIONS DES MÉDIAS ET DES GENRES}

Par ailleurs, les attentes du lecteur peuvent être influencées par les figurations associées à un média et à un genre textuel. La presse en général, par exemple, la presse d'information ou d'opinion en particulier, la brève, l'interview, l'analyse ou l'éditorial constituent des médias et genres renvoyant à une longue tradition et à des imaginaires partagés.

Faits, opinions, fictions ?

Quelques figurations du texte journalistique

Il est impossible de retracer ici l'évolution des figurations de la presse de façon détaillée. Je renvoie au livre de Bernard Miège [2010] consacré à l'« espace public contemporain ", dans lequel il étudie à la fois l'évolution historique des relations complexes entre l'espace public et les médias, et les transformations récentes apportées par les dispositifs numériques. Si, 
comme l'affirme Bernard Miège [2010, 35], la représentation de l'espace public est indissolublement liée au projet démocratique, la demande d'une information " universelle » et "vraie » s'est fait entendre dès le Second Empire. En privilégiant " les faits sur les opinions », cette demande " exprime la volonté de poursuivre le processus d'une opinion publique éclairée », de " défendre le sérieux contre la frivolité, la citoyenneté contre l'indifférence, l'instruction contre la distraction».

L'association fréquente entre "vérification » et « Internet », « information », « factualité » et « hyperlien » pourrait donc s'expliquer par cette attente d'un médium "transparent », investi de la fonction de relater des « faits ». Comme l'a affirmé Tom Wolfe [1973], le journalisme papier semble associé à une certaine présomption d'objectivité et de vérité. D’un journalisme numérique, les lecteurs interrogés semblent attendre de même qu'il fournisse, via l'hyperlien, des preuves aux affirmations avancées dans le texte géniteur, qu'il renvoie aux sources utilisées par le journaliste, ou qu'il complète les données présentées par d'autres données, factuelles et vérifiables.

Or, comme le fait remarquer Bernard Miège [2010, 34], « si l'on donne aux journaux la fonction d'enregistrer les faits sans les discuter », on oublie que le principe de l'espace public s'applique aussi aux opinions. Même en dehors du fait qu'il est difficile de distinguer strictement entre faits et opinions à partir du moment où s'opèrent, dans l'écriture, une sélection, une hiérarchisation et une présentation des données, l'on doit selon Miège accepter que des divergences s'expriment dans les journaux « lorsque les opinions sont en effet diverses, parce qu'elles correspondent à ses intérêts eux-mêmes divers » (34).

Certains lecteurs de la presse papier en semblent bien conscients, comme le montre une étude qualitative menée par Dialego pour les États généraux de la presse écrite en 2008 avec 200 participants [Faure, Sassinot-Uny, 2008]. Via une plate-forme de discussion numérique, des hommes et femmes âgés de plus de 15 ans, vivant en France et se déclarant eux-mêmes lecteurs de la presse, ont été confrontés à des thèmes de discussion autour de leurs usages de la presse écrite, leur opinion sur la presse et leurs attentes. La synthèse des résultats effectuée par Nadine Faure et Laetitia Sassinot-Uny fait certes émerger une vision utilitaire de la presse. Beaucoup de lecteurs insistent sur un manque de temps qui les oblige à parcourir rapidement les textes proposés. Néanmoins, une majorité des lecteurs déclarent que la presse fait partie des "valeurs démocratiques ». Elle est associée à la notion de « liberté de parole » et de « liberté 
d'opinion », même si certains expriment aussi des doutes quant à son indépendance. Sur ce point, les lecteurs accordent plus de confiance à la presse écrite qu'aux autres médias comme la télévision, la radio ou l'Internet, et cela même si les études sur les pratiques culturelles des Français ${ }^{13}$ montrent que la lecture de la presse papier nationale et régionale est en baisse quasiment constante depuis 1973 [voir aussi Donnat, 2011, 28].

Un « paradoxe » s'installe, comme le formulent Nadine Faure et Laetitia Sassinot-Uny [2008, 5], « entre les lecteurs qui sont à la recherche d'une information objective et aussi neutre que possible et ceux qui au contraire sont à la recherche d'opinions et de positionnements afin de confronter des points de vue et mener à la réflexion ».

La figuration d'une "presse d'opinion » proposant la juxtaposition dialogique de points de vue est donc projetée par certains lecteurs, certes moins nombreux, sur les éditions numériques de journaux ou magazines. L'hyperlien se trouve dans ce contexte investi de l'espoir de mettre en branle la "prétention de vérité ». Il devient porteur de notions de contradiction, d'ouverture et de pluralisme [Huesca, Dervin, 2003, 282]. Lorsque Andrew Nibley [2000, 37-38] affirme que le public attend désormais du journaliste une résistance aux « interprétations manichéennes et idéologiques » de la réalité, il énonce une figuration de l’Internet comme « espace public » élargi, où le lecteur est appelé à se frayer lui-même son chemin à travers les points de vue juxtaposés. Entend-il par là aussi que la fonction du journaliste consiste principalement à créer des liens entre des données sans prendre lui-même position ? Ou veut-il plutôt dire que le journaliste, tout en exposant son point de vue, doit utiliser l'hyperlien pour montrer que d'autres points de vue existent ? Ces figurations dialogiques de l'hyperlien rencontrent-elles les attentes du lecteur, comme Andrew Nibley le présume?

L'expérience pédagogique précitée ne peut évidemment pas être considérée comme représentative des figurations du journalisme numérique dans la société française. Elle donne un aperçu possible. La plupart des étudiants ayant participé à l'expérience s'attendaient certes à ce que les textes reliés à un article journalistique fournissent des compléments d'information factuels. Quelques sujets ont néanmoins évoqué la possibilité d'une mise en relation dialogique d'arguments et de contre-arguments. L'émergence du paradigme de la lecture sociale contribuera peut-être à renforcer cette figuration dialogique. Peu après la constitution de mon

13. Enquêtes sur les « Pratiques culturelles des Français », op. cit. 
corpus journalistique qui inclut en outre des articles de lexpress.fr, le groupe a décidé d'autoriser désormais les commentaires de lecteurs à la fin des articles. Pour marianne2.fr et lepost.fr, l'ouverture au débat avec les lecteurs s'était effectuée déjà bien avant.

Malgré une institutionnalisation progressive des «blogs d'opinion », et une ouverture de plus en plus large de la presse numérique aux réseaux sociaux accompagnée parfois de discours enthousiastes [voir par exemple Jean-François Kahn] ${ }^{14}$, il ne faut pas sous-estimer l'impact des représentations attribuant à la presse le rôle d'enregistrer et de transmettre l'information de la façon la plus neutre et transparente possible. Ces figurations se reflètent dans l'idée d'un hyperlien comme lien efficace entre des données factuelles.

Face à un horizon d'attente encore fortement marqué par une figuration informationnelle de la presse, se pose aussi la question de l'acceptation possible d'une irradiation iconique apportée par la manipulabilité du texte, qui non seulement renforcerait des attentes de "révélation " et de « vérité » [Gervais, 2006], mais irait jusqu’à expérimenter dans ses dimensions immersives.

Selon Ken Pimentel et Kevin Teixeira [1993, 15], la suspension de l'incrédulité est une condition sine qua non de l'immersion. Qu'elle soit valorisée ou dénoncée, l’immersion est généralement associée à certains genres textuels : le roman et le cinéma narratifs, et plus récemment le jeu vidéo. En avançant que la non-fiction est proposée pour qu'on y croie alors que la fiction est proposée pour " faire-croire ", Gregory Currie [1990, 91-93] pose le problème de façon polémique, et évidemment discutable. Les débats virulents autour de la "réinterprétation » de l'affaire Villemin par Marguerite Duras, publiée par Libération en 1985, ne sont pourtant qu'un exemple démontrant la vitalité d'une distinction toujours opérationnelle entre fiction et faits [voir Saemmer, 2006]. Le rôle attribué à la presse par grand nombre de lecteurs semble décidément être celui de relater des faits, et cela même lorsqu'un journal donne la parole à un écrivain. Les débats récurrents autour de la « fictionnalisation » de faits divers montrent que beaucoup de lecteurs ne sont pas prêts à accepter les prémisses du « Nouveau Journalisme », défendues entre autres par Tom Wolfe dès les années 1970 [1973, 31]. L'auteur avait plaidé pour l'introduction d'éléments fictionnels dans la description du réel afin de pallier

14. Fanny Bragard, « Jean-François Kahn : "la presse ne correspond plus aux attentes de ses lecteurs" », 5 juin 2010, [en ligne] : < http://blogs.mediapart.fr/edition/metz-dans-les-coulisses-delete-du-livre/article/050610/jean-francois-kahn-la-presse-ne >. 
« au manque d'adresse imaginative » du journalisme traditionnel et de provoquer un nouvel investissement du lecteur.

Dans l'hyperlien immersif et l'animation textuelle, cette forme de journalisme pourrait trouver de nouveaux procédés rhétoriques. Leur utilisation dans la presse peut pourtant susciter de fortes réticences. Le fait qu'interactivité et ludicité se trouvent étroitement associées dans de nombreux discours consacrés à l'Internet et à l'hyperlien [voir Saemmer, Maza, 2011] n'aide sans doute guère à balayer ces réticences, et peut suggérer l'idée que l'hyperlien immersif et l'animation textuelle constituent avant tout des outils de l'infotainment : une forme d'énonciation ludique dans laquelle, comme l'affirme Ionna Vovou [2008, 230-33], on retrouve peu les marques de l'espace public de débat fondé sur la raison et le logos.

Le reproche de l'infotainment a, par exemple, été adressé aux immersives news expérimentées par certains médias américains, qui vont certes beaucoup plus loin dans le simulacre de référent [voir Nolan, 2003] en travaillant avec de l'image 3D. D'un côté, l'immersion corporelle du lecteur dans les événements représentés a l'avantage de créer un surplus d'empathie (rappelant les objectifs de certains serious games). D'un autre côté, cette immersion comporte le risque d'une fictionnalisation de la réalité. C'est peut-être la raison pour laquelle même l'exploration de l'hyperlien immersif et de l'animation textuelle, procédés rhétoriques pourtant moins périlleux grâce à leur caractère plurisémiotique (le mot " interrupteur », même manipulable, reste un mot), est rare dans le corpus journalistique qui sera étudié en profondeur au chapitre III, " Figures de la lecture du texte numérique dans les discours informatif et argumentatif », p. 116.

\section{Immersion, réflexivité, renarrativisation ? Quelques figurations de I'hyperfiction}

Grâce à l'association fréquente entre fiction et immersion, les figurations du texte littéraire numérique pourraient être considérées comme plus spontanément accueillantes pour la manipulation et l'animation « immersives ». Avant l'examen du rôle potentiel de ces procédés rhétoriques dans un genre textuel qui a rapidement été appelé " hyperfiction », se pose pourtant la question plus générale de savoir si le lecteur est prêt à lire un texte littéraire, de surcroît narratif, sur un support numérique.

Dans l'enquête de 2006 (voir note 19, p. 60), 40,5\% des sujets ont affirmé lire un texte numérique seulement en diagonale. Beaucoup ont associé leur pratique à la recherche d'informations; la possibilité d'une 
lecture immersive de textes de fiction n'a été évoquée dans aucun questionnaire. Dans beaucoup de publications académiques [Carr, 2008 ; Gaschke, 2009], la lecture attentive ou immersive d'un récit est considérée comme incompatible avec les caractéristiques du numérique.

Ces dernières années, de nouvelles formes de fictions interactives et multimédias ont pourtant émergé sur Internet, sur tablettes et téléphones portables; ces productions pourraient faire évoluer les figurations du texte narratif et favoriser des pratiques de lecture plus attentives, exhaustives et éventuellement immersives. Comme le montre le mémoire de Master 2 présenté en septembre 2012 par Nolwenn Tréhondart ${ }^{15}$, les éditeurs de ces fictions anticipent en tout cas sur le fait que l'animation et la manipulabilité du texte et de l'image numériques réussissent à captiver le lecteur pour l'immerger plus profondément dans les histoires racontées.

Dans ce contexte hautement évolutif, il est difficile de circonscrire avec précision les figurations de l'hyperfiction. Pour étudier l'influence de ces figurations sur la lecture, il est d'abord important de considérer que l'association entre interactivité et narration s'est trouvée mise en question dans de nombreux discours académiques, et cela non seulement à cause de la " présomption d'information » avec laquelle le lecteur approche souvent les textes numériques, mais pour des raisons structurelles.

Les « ludologistes » comme Espen Aarseth [1997] sont allés jusqu’à contester la narrativité des jeux vidéo : la signification narrative exigerait une linéarité et une unidirectionnalité du temps et de la causalité, alors que les jeux vidéo seraient fondés sur un système de branches « multilinéaires » permettant au lecteur de faire des choix. Rejaillit dans ces discours la figuration topographique de l'hypertexte exposée plus haut. Y transparaît aussi une certaine idée de ce qu'est la « signification narrative ».

S’inscrivant dans une longue tradition de la théorie de la narration remontant à l'Antiquité, Paul Ricœur [1983, 130] a avancé que « suivre une histoire, c'est avancer au milieu de contingences et de péripéties sous la conduite d'une attente qui trouve son accomplissement dans la conclusion » : une histoire doit être plus qu'une énumération d'événements ; elle doit organiser ces événements dans une " totalité intelligible ». Citant les réflexions de Paul Ricœur qui fait du temps une donnée essentielle de la narrativité, Bertrand Gervais et Nicolas Xanthos [2003] ont mis en avant deux caractéristiques du récit classique : la fin et la séquentialité.

15. Nolwenn Tréhondart, " L'émergence de nouveaux produits éditoriaux pour tablettes et téléphones portables ", Mémoire de Master 2 recherche, sous la direction d'Alexandra Saemmer : université Paris 8, 2011-2012. 
L'hypertexte mettrait en danger ces deux dimensions « indispensables »: la conclusion serait plus ou moins suspendue, et la séquentialité se trouverait remplacée par « une logique du fractionnement ». Gervais et Xanthos ont tiré de leurs réflexions des conclusions pessimistes concernant la lisibilité des hyperfictions. Umberto Eco [2003] a affirmé de même qu'un texte narratif ne devrait pas avoir comme objectif d'imiter une « encyclopédie », mais de réduire le nombre de possibilités combinatoires.

Encore une fois, il ne s'agit pas ici de se prononcer sur la validité de ces figurations de l'hyperfiction, mais d'étudier leurs répercussions possibles sur les pratiques de lecture. Dans cet objectif, il faut d'abord mentionner que la figuration du texte narratif, fondée sur la séquentialité et l'enchaînement linéaire, n'est pas la seule ayant imprégné les discours théoriques et les créations littéraires au xxe siècle [voir aussi Saemmer, 2007]. Un certain nombre de théoriciens et d'auteurs ont, notamment lors de la seconde moitié du $x^{e}$ siècle, considéré la structure logicotemporelle du récit « classique » comme inapte à rendre compte de la complexité des processus historiques, politiques et technologiques du monde contemporain. Les auteurs du nouveau roman ont vu dans la " conclusion classique », lors de laquelle les intérêts des personnages se réconcilient, le reflet d'une société sclérosée. Pour empêcher le lecteur de fermer le livre sur un sentiment de complétude, certes rassurant mais déresponsabilisant, le roman ne devait donc avoir désormais ni début ni fin. « Tous les éléments techniques du récit - emploi systématique du passé simple et de la troisième personne, adoption sans condition du déroulement chronologique, intrigues linéaires, courbe régulière des passions, tension de chaque épisode vers une fin, etc. -, tout visait à imposer l'image d'un univers stable, cohérent, continu, univoque, entièrement déchiffrable », a observé Alain Robbe-Grillet [1963, 36-37]. Le nouveau roman devait mettre en question ces fondements. Il incombait maintenant au lecteur de créer ses parcours à travers les « fragments » proposés.

Robert Musil [1978, 53] a rêvé d'un roman sous forme de surface entretissée, présentée de façon non reliée. Alain Robbe-Grillet [1970] a tissé, dans Projet pour une révolution à New York, un réseau d'actions et de motifs simultanés dont les interconnexions ne peuvent plus être situées avec certitude sur un axe temporel, ni s'enchaîner selon des principes de cause à effet. Claude Simon [1977, 41] a défini les structures narratives du nouveau roman de la manière suivante : "Si l'enchaînement des épisodes et leur aboutissement n'ont aucune valeur exemplaire, je ne vois plus, non seulement pour la phrase mais pour le texte entier du roman, qu'à 
chercher une construction qui tienne debout non pas en référence à telle "vraisemblance" psychologique ou sociale, mais en référence au texte luimême, à la logique de la langue travaillée, à sa justesse qui est, comme le disait Flaubert, d'ordre musical ».

Jean Clément [1994], Bernard Magné [2000] et moi-même [2002, 2003] avons rapidement proposé un rapprochement entre les mouvements d'avant-garde du $x^{e}$ siècle et l'hyperfiction émergeante. En avançant que dans la littérature hypertextuelle et programmée, « le refus de la tentation du sens peut être aussi, dans certains cas, le signe d'une contestation de l'ordre institué par la tradition littéraire et par la langue elle-même » (3), Jean Clément a inscrit les créations littéraires numériques dans une filiation qui avait transgressé la taxinomie du récit classique bien avant l'arrivée de l'ordinateur.

Dans l'hyperfiction, le lecteur se trouve, selon Jean Clément [2004], placé devant des choix suscitant de la dissonance cognitive : quel lien suivre ? Comment anticiper sur le fragment à venir ? Comme Bertrand Gervais et Nicolas Xanthos [2003], Jean Clément en a conclu que la linéarité discursive était atteinte par l'hypertexte : celui-ci favoriserait une écriture fragmentaire, elliptique. Dans les années 1990, beaucoup de théoriciens français et américains de l'hypertexte ont pourtant valorisé l'hyperfiction justement parce qu' elle présente une figuration " anti-aristotélicienne », délibérément dirigée contre la linéarité et l'unité narratives [Landow, 1997, 181 ; Jennings, 1996, 349 ; Amerika, 2004, 9]. Les auteurs saluaient la désorientation, voire la frustration du lecteur face à des hypertextes labyrinthiques. Entre la traversée indéfinie de boucles (appelées " apories ») et les moments de révélation [appelés " épiphanies » par Aarseth, 1997, 91], le parcours d'une hyperfiction se voulait avant tout errance. Certains auteurs [voir Bolter, 1991] ont opposé avec fermeté hypermédialité et immersion : alors que l'immersion serait recherchée par le lecteur naïf en quête de distractions, l'hypermédialité serait, par nature, propice à la lecture distanciée et réflexive.

Dès qu'on abandonne la figuration " topologique » de l'hypertexte et que l'on se penche sur l'hyperlien en tant que mot ou image pointant vers un texte relié, l'association entre hypertexte et non-séquentialité paraît cependant moins évidente. Dans leur typologie des hyperliens, Norton, Zimmerman et Lindeman [1999] ont déjà avancé que l'hyperlien peut bien avoir une fonction temporelle, qui rapproche l'hyperfiction des schémas narratifs du récit " traditionnel ». L' « anachronophora » relie par exemple au texte géniteur « la séquence précédant une étape située à un moment 
donné ». La " catachronophora » propose l'exploration de la séquence suivante. En examinant le rôle de l'hyperlien dans l'hyperfiction, MarieLaure Ryan [2006, 109] constate de même que la relation établie par un hyperlien entre des textes peut ramener le lecteur en avant ou en arrière sur l'axe temporel de l'histoire racontée. Dans Matières textuelles sur support numérique [2007], j'ai parlé de « liens proleptiques », " analeptiques » et " chronologiques » pour désigner ces potentiels d'action anticipés par l'hyperlien (voir aussi chapitre III, " Figures de la lecture et formesmodèles de la page-écran dans le discours narratif », p. 183).

Ces potentiels d'action ne mettent pas obligatoirement en cause la taxinomie du récit " classique ». Mais dans les discussions académiques autour de l'hyperfiction, ce n'est pas seulement la possibilité, c'est la légitimité même d'une telle satisfaction des attentes « traditionnelles » vis-àvis du récit qui a fait débat. Beaucoup d'auteurs adhérant à la figuration d'un récit numérique fragmentaire et dissociatif ont valorisé la désorientation du lecteur et la perte de repères [Landow, 1997 ; Joyce, 1993]. Cette figuration était tellement dominante que Marie-Laure Ryan s'est demandée si une hyperfiction travaille contre le médium lorsqu'elle établit de la cohérence [2006, 330]. Certains chercheurs comme Renée Bourassa, en revanche, ont plaidé plus récemment pour la remise en question de cette association systématique entre désorientation et hypertexte, revalorisant la cohérence et l'immersion [Bourassa, 2010, 62].

Une troisième voie, « intermédiaire » au sens fort du terme, pourrait se dessiner pour l'hyperfiction à partir du moment où elle s'inscrit dans l'idée d'une "renarrativisation " [Blanckeman, 2000, à la suite de Aron Kibedi Varga]. Des auteurs comme Jean Echenoz ou Pierre Michon, représentants de la renarrativisation dans le domaine papier, n'essaient pas de restaurer le roman " classique » fondé sur un schéma aristotélicien. Il s'agit plutôt de montrer " comment une fiction s'élabore sur sa propre ruine ». Les personnages, "intégrés à l'intérieur d'une ou de plusieurs intrigues, s'affirment sans s'affermir ». Le roman " produit de la fiction et surligne cette production, énonce du romanesque et le dénonce comme tel » (15-17). Ainsi devient-il, selon Bruno Blanckeman [2000], un médiateur pour temps flottants. Il préfigure un lecteur qui accepte par exemple de se prendre au jeu de la fiction et de l'immersion « tout en sachant » : comme le spectateur d'un film 3D qui, de temps à autre, enlève ses lunettes.

Le lecteur est-il prêt à adopter ces pratiques de lecture mi-immersives mi-réflexives, que l'on pourrait qualifier avec Marie-Laure Ryan [2001, 
351] de "medium-aware" (conscientes du dispositif)", face au texte numérique ? Le lecteur devrait dans ce cas accepter de s'impliquer intensément dans l'expérimentation du texte à la fois lisible, visible et manipulable, et élaborer des hypothèses d'interprétation face à des expériences de lecture restant potentiellement en suspens, entre cohérence et décohérence, confirmation et mise au défi de ses attentes. Je discuterai plus amplement cette préfiguration de pratiques au chapitre III en analysant en détail une hyperfiction.

Mal nécessaire, terrain de jeu?

Quelques figurations de la bannière publicitaire

Si la question du niveau d'acceptation par le lecteur se pose jusqu'à un certain point pour l'hyperfiction, elle s'impose de façon cruciale pour le troisième corpus du chapitre III, constitué de bannières publicitaires et d'annonces commerciales. Quelles sont les figurations des bannières publicitaires sur Internet ? De façon spontanée, l'on pourrait répondre qu'elles sont mal considérées, et que l'effort du lecteur consiste avant tout à ne pas les lire. Laurent et Kapferer [1986] ont montré à quel point l'intérêt pour la publicité est faible, notamment à cause de la quantité de messages à laquelle les sujets se trouvent quotidiennement exposés. Selon Drèze et Zufryden [2000], le lecteur sur Internet prête une attention très réduite aux bannières publicitaires : il les considère comme étrangères à sa " tâche principale ", que celle-ci consiste à chercher des données factuelles ou à regarder un film. L'idée qu'on « n'aime pas la publicité sur Internet » semble être un allant-de-soi.

Didier Courbet [2004, 30] a souligné le rôle des émotions dans la réception : «Ainsi, une marque qui occupe un espace jugé trop important sur un écran de télévision au moment d'un match de football est susceptible de provoquer un phénomène de rejet et la rupture du contrat médiatique (le spectateur éteint la télévision) ». Caroline de Montety [2004, 84] précise que notre époque est particulièrement " encline à la dénonciation de la publicité ». Dans la ligne de mire des critiques se trouve en particulier le « ciblage comportemental » : la collecte d'informations sur les parcours de navigation des lecteurs visant à proposer des publicités adaptées.

16. Il me semble plus juste dans ce contexte de traduire le terme "medium" par " dispositif ", concept pour lequel n'existe pas vraiment d'équivalent en anglais. 
Malgré ce désintérêt, voire ce rejet de la part du lecteur, la publicité en ligne représente un facteur économique important. Selon une étude menée par McKinsey pour IAB Europe, les annonceurs en Europe ont consacré en 2009 14,7 milliards d'euros à la publicité en ligne. Même si cette étude révèle que les usagers de l'Internet accordent de l'importance à la protection de leur vie privée, $70 \%$ des sujets interrogés attribuent encore plus de valeur aux services offerts gratuitement grâce à la pub. Le diffuseur fournit aux lecteurs de l'information, des produits culturels et du divertissement. En échange, ceux-ci sont exposés aux discours persuasifs de la publicité, et semblent majoritairement prêts à accepter ce " marché » comme un mal nécessaire - peut-être pensent-ils que leur faible intérêt pour la publicité permettra d'échapper à son influence.

En effet, la moyenne du taux de clic sur la publicité en ligne a toujours été faible. De $1 \%$ en 1999, elle est passée à 0,15\% en 2006 : Lendrevie [2006] avance l'hypothèse que le lecteur a acquis une expertise qui le rend capable de « moins voir » les publicités numériques, et donc de moins cliquer dessus. Bien que, selon une étude d'eMarketer en 2010, 60 \% des répondants recourent encore aux taux de clics pour mesurer l'efficacité de leurs campagnes interactives sur le Web, une prise de conscience s'est installée ces derniers temps quant à l'insuffisance de cette mesure. Cette intuition a constitué l'un des points de départ des recherches menées sur la réception de la publicité numérique [Courbet, 2004].

Les bannières, souvent placées en haut de l'écran, entrent dans la partie périphérique du champ de vision fovéale : celui-ci se situe à un angle compris entre $0^{\circ}$ et $2^{\circ}$ du point de focalisation visuelle. Selon Anand et Sternthal [1991], ces discours persuasifs influencent le comportement du lecteur même s'il ne reconnaît pas son exposition au message comme telle. Shapiro et Krishnan [2001] s'appuient notamment sur des recherches concernant la " mémoire implicite » pour démontrer que les bannières ont une certaine " efficacité », même si l'attention du lecteur n'est pas focalisée dessus. Courbet, Borde et al. [2004] ont approfondi ces recherches sur la persuasion « sans conscience ", montrant que le récepteur peut être amené à acheter des produits suite à une exposition, même furtive, à la publicité.

Courbet [2004, 30] distingue entre trois situations de réception. La première est le traitement des messages persuasifs avec une forte allocation d'attention. Dans la deuxième situation, le lecteur a également conscience de la présence du message persuasif, mais lui alloue un niveau d'attention moyen. Dans la troisième situation, le récepteur n'a pas conscience du stimulus persuasif. Dans ce dernier cas, Courbet [2004, 38] 
recommande de ne plus parler d'une " coproduction de significations ", mais d'« effets affectifs et non conscients où l'activité du sujet, non verbalisable, n'amène pas à la construction de significations mais conduit, tout de même, à engrammer des traces mnésiques et à produire des effets comportementaux ».

Est-ce que les bannières, même perçues de façon non consciente, provoquent des décisions d'achat parce qu'elles font appel à des couleurs, symboles et logos utilisés sur d'autres supports de communication : l'affichage dans l'espace urbain, le spot télévisé ? Est-ce qu'elles arrivent à atteindre le lecteur à un niveau infra-conscient par la mobilisation de certaines formes-modèles, comme des mouvements et des gestes qui, grâce à leur caractère iconique, peuvent renvoyer à des référents d'expérience ? Je reviendrai sur ces questions au chapitre III, « Figures de la lecture dans le discours persuasif », p. 217.

Alors que, d'après les études précitées, peu de lecteurs prêtent une attention consciente aux publicités, les créateurs s'attendent, selon une étude qualitative menée par Didier Courbet et Marie-Pierre FouquetCourbet [2005], à ce que chaque procédé créatif provoque des « effets spécifiques » chez le lecteur. Les associations faites par les professionnels entre effets et réactions renvoient à des systèmes de " représentations sociocognitives » organisées sur la manière dont Internet influence les usagers. Les auteurs citent en guise d'exemple l'affirmation que "le clignotement attire l'attention du récepteur ».

Nicole Pignier [2005] a étudié un corpus de bannières publicitaires suivant une approche sémiotique. Elle distingue entre plusieurs formes de «multimodalité » dans les bannières : alors que le défilement d'un texte dans un espace restreint ne sert souvent qu'à le rendre plus lisible, des images, couleurs et " typographies animées » peuvent, selon Pignier, « illustrer » le contenu, mobilisant des figures comme l'hypotypose, impliquant un regain de mémorisation et attirant l'attention du lecteur par la " technique de l'insistance polysensorielle " (528). Au clignotement de l'énoncé " cliquez ici » correspondrait ainsi, « sans ambiguité », le signifié « attention, urgent, important » (529). Certains énoncés du corpus de bannières étudié par l'auteur seraient caractérisés par une " rhétorique du miroitement » (529) : texte et mouvement rentrent dans une " complicité » pour faire émerger un "énoncé facile à décoder », et se caractérisent par les figures de la redondance, de la surenchère et de l'hyperbole. Un énoncé peut, selon l'auteur, également être animé pour « ouvrir un espace symbolique dont la rythmique suscite une participation imaginaire et 
sensible de l'internaute ": l'énoncé deviendrait dans ce cas une " source sensible », qui pousse l'internaute à « s’intriguer » (526).

Enfin, certains énoncés s'animent, selon Nicole Pignier, pour « ouvrir un espace en devenir, sensible et narratif » dans lequel l'internaute est invité à un "partage poétique » de scènes "imaginaires à inventer et à réinventer », afin de se " dépayser » et " s’immerger » (532). Dans ces cas, précise Nicole Pignier, « l'instance d'énonciation comme l'instance de réception se définissent par un rapport aux choses et au langage plus lent et plus impliquant ». Le lecteur doit être " disponible » pour " accueillir les tensions et effets plastiques »(532). Pignier présume que de telles créations publicitaires peuvent rompre avec « l'image institutionnelle distante » d'une marque (533) ; elles exigent pourtant un lecteur prêt à se laisser prendre au jeu, comme je le montrerai maintenant au chapitre III, à travers l'étude de corpus. 


\section{CHAPITRE III FIGURES DE LA LECTURE DU TEXTE NUMÉRIQUE}




\section{FIGURES DE LA LECTURE DU TEXTE NUMÉRIQUE}

L' objectif de la rhétorique présentée dans ce livre est d'étudier les figures du texte numérique comme des potentiels d'action. Je rappelle que la figure est donc définie non pas comme un procédé au sens immanent, mais comme une préfiguration de pratiques de lecture. En identifiant ces figures de la lecture, la rhétorique étudie à la fois les procédés déployés lors de l'interprétation et de la représentation du réel par les auteurs de textes numériques, et propose au lecteur des clés pour appréhender, décrypter et jouir de ces outils.

Le processus de réception est considéré comme un champ de tensions entre ces potentialisations opérées par le texte, et leur actualisation forcément partielle par le lecteur. Les figurations (c'est-à-dire les imaginaires et représentations) individuelles et socialement partagées du texte, du dispositif et du genre identifiés au chapitre précédent orientent l'acte de réception, en amont et pendant le processus de lecture, et doivent donc être prises en compte dans chaque étape de l'analyse rhétorique de corpus qui suivra. La signification, toujours dynamique, du texte numérique émerge de la convergence entre le potentiel d'action reposant sur les répertoires du texte, ses figures de la lecture et formes-modèles de la page-écran qui engagent le lecteur vers un point de vue, la " médiation » de ce point de vue par le dispositif, et la réception orientée par l'horizon d'attente extra-textuel du lecteur.

Cette rhétorique est donc par définition une science à prétention limitée. Certes, l'horizon d'attente peut être sondé par des études empiriques (voir chapitre précédent). Celles-ci sont pourtant historiquement et socialement situées. Certes, le potentiel d'action du texte numérique sur la page-écran et dans son dispositif peut être circonscrit de la façon la plus précise possible. Cette description sera pourtant toujours médiée par le point de vue personnel du lecteur-interprète. L'étude des discours académiques consacrés aux bannières publicitaires en ligne montre par excellence à quel point chaque analyse repose d'abord sur une « décision sémiotique » [Klinkenberg, 1996, 83] : la lecture d'un couplage entre un texte et un mouvement dépend fortement de la focalisation de l'attention du lecteur sur le texte, le mouvement 
ou sur un autre élément de la page-écran. Même si la rhétorique du texte numérique assume donc pleinement son statut de science inexacte, la sensibilisation aux couches de médiation du texte paraît néanmoins un enjeu essentiel pour la recherche, l'écriture et l'édition numériques, et pour l'enseignement d'une culture numérique réflexive et critique.

Au regard des statistiques récentes consacrées à l'évolution des pratiques de lecture, il paraît probable qu'elle s'effectuera de plus en plus sur des dispositifs numériques, qu'il s'agisse de pratiques orientées vers la recherche d'information, l'apprentissage ou l'immersion dans des mondes fictionnels. Chercheurs, auteurs, lecteurs et apprenants ont besoin de repères pour cerner les enjeux communicationnels du texte, afin de les mettre en œuvre dans leurs pratiques professionnelles et personnelles. La rhétorique du texte numérique a comme objectif de proposer quelques-uns de ces repères. Montrant comment le texte préfigure les pratiques de lecture en anticipant sur les pratiques, et comment le lecteur y répond potentiellement en mobilisant des figurations personnelles et socialement partagées, elle développe à la fois des outils de défense contre les effets de persuasion et de manipulation du texte mobilisés par des structures de domination, et propose des repères pour goûter à un nouveau plaisir du texte numérique.

Pour atteindre cet objectif, il paraît essentiel de circonscrire maintenant avec précision l'horizon intra-textuel : la part qui, dans la communication entre texte et lecteur, revient au texte numérique avec ses spécificités. Avant même d'être activé, l'hyperlien est certes approché avec des imaginaires : le lecteur investit sa présence d'un certain nombre d'idées préconçues. Par ses figures de la lecture, le texte participe pourtant à son tour à la projection, la constitution et l'évolution de ces attentes.

Certains textes géniteurs, appelés ainsi lorsqu'ils contiennent des hyperliens, préparent par exemple le terrain au texte relié en annonçant ce qui reste à découvrir. Les attentes du lecteur peuvent ensuite se trouver confirmées par le texte relié, ou alors être mises au défi par des découvertes plus ou moins déconcertantes. Le niveau de contiguité entre le texte géniteur et le texte relié constitue un élément important de l'hyperlien en tant que figure de la lecture. Avant de présenter une typologie détaillée de ces figures, je propose de considérer un exemple type.

Imaginons un énoncé qui se trouve dans un article d'actualité publié sur le site du magazine lexpress.fr. L'article commencerait ainsi : "Selon Europe 1, François Fillon a démenti toute volonté de candidater à la présidentielle ». L'hyperlien, dont la présence est indiquée par un soulignement, est donc posé sur le nom d'un autre média, Europe 1. Plusieurs études 
empiriques (voir chapitre II) laissent penser que les lecteurs approchent majoritairement l'hyperlien avec une présomption d'information. Dans cet exemple, c'est le nom de la source de l'information qui se trouve souligné. Les attentes sont donc potentiellement orientées vers la découverte de cette source ; elles pourraient être paraphrasées par la question : "Le texte relié va-t-il confirmer l'existence de cette information sur le site d'Europe 1 ? " L'affichage effectif de la page-écran du site europel.fr contenant l'information du démenti répondrait à ces attentes suscitées.

Imaginons maintenant que l'hyperlien se trouve inséré dans l'énoncé de la manière suivante : "Selon Europe 1, François Fillon a démenti toute volonté de candidater à la présidentielle » : ce n'est plus la source de l'information, mais l'information elle-même qui se trouve hyperliée. Cet hyperlien pourrait susciter non pas tellement l'attente de pouvoir vérifier la source, mais de recevoir des compléments d'information. Cette attente se paraphraserait donc par des questions comme : «Pourquoi François Fillon a-t-il démenti sa volonté de candidater ? Dans quelles circonstances ? » Si la page du site d'europe1.fr confirme le démenti de François Fillon, la présomption d'information de beaucoup de lecteurs se trouverait de nouveau confirmée, même si la lecture d'un complément d'information demande éventuellement une attention plus soutenue que la simple vérification d'un renvoi à la source.

Examinons maintenant plus précisément l'énoncé « a démenti toute volonté de candidater à la présidentielle ». Le mot « démenti » renvoie à la rumeur d'une éventuelle candidature de François Fillon à la présidentielle. Il est tout à fait possible d'envisager que le texte relié, au lieu de confirmer et d'expliciter le démenti, donne accès à cette rumeur initiale. Un tel hyperlien peut défier la présomption d'information du lecteur. Compte tenu du répertoire d'un mot comme " démenti », il paraît néanmoins possible que le lecteur se demande : "Qui a lancé la rumeur de la candidature, et pourquoi ? ", et qu'il s'attende à un croisement de points de vue sur la question de l'éventuelle candidature ${ }^{1}$ : attente qui lui permettra de ne pas se sentir complètement désorienté par le texte relié. Peut ici entrer en compte le fait que L'Express ne se présente pas que comme un magazine de news, mais comme un média relevant du journalisme d'opinion. Un hyperlien mettant en relation des informations divergentes pourrait

1. Lors de l'étude exploratoire auprès des étudiants de l'université Paris 8, l'énoncé hypertextualisé « présumé innocent » dans l'article consacré à John Galliano a suscité des attentes de prise de position fortes, voire d'une présentation d'arguments et de contre-arguments, car il porte en lui-même un potentiel d'action dialogique. Voir le chapitre II. 
répondre à la figuration d'un tel média intervenant activement dans les débats d'actualité. Même au cas où il répond donc aux attentes de certains lecteurs, un tel hyperlien s'appréhende pourtant moins rapidement qu'un hyperlien à potentiel d'action informationnel.

Imaginons enfin que l'hyperlien se trouve posé sur le nom de « François Fillon » et renvoie à un article du canardenchaine.fr révélant que l'homme politique a utilisé un avion de l'ancien régime Moubarak pendant ses vacances en Égypte. Un lecteur approchant cet hyperlien avec une présomption d'information ne s'attend probablement pas à cette découverte. Même un lecteur avec des expectatives dialogiques (s'attendant alors à un croisement de points de vue par hyperlien) peut être surpris. Un tel hyperlien demande donc un effort d'interprétation soutenu.

Dans l'exemple cité, ce processus interprétatif pourrait reposer sur les éléments suivants : l'article de L'Express comme celui du Canard enchaîné renvoient au même ancien Premier ministre, François Fillon. Les deux textes traitent de son rôle en tant qu'homme d'État. Le texte géniteur évoque un démenti sans l'expliquer; le texte relié, une révélation mettant en cause les agissements de l'homme sur le plan déontologique. La rétroprojection de cette révélation sur le texte géniteur pourrait donc mettre en question la légitimité de François Fillon de se présenter à la présidentielle, indépendamment de sa volonté ou non de candidater. Non seulement le lecteur n'est pas préparé à cette révélation par le texte géniteur, mais le texte relié défie aussi potentiellement ses attentes ; le texte relié mine littéralement le texte géniteur en mettant en cause la crédibilité de François Fillon. Cet hyperlien peut donc être interprété comme la trace d'une volonté du journaliste de subvertir l'affirmation de François Fillon tout en déléguant l'acte de dénonciation au Canard enchaîné. La lecture d'un tel hyperlien ne demande pas qu'un travail d'interprétation; elle nécessite un examen de la relation ainsi créée, entre deux médias aux stratégies et positionnements différents.

La subtilité de cette figure de la lecture repose par ailleurs sur le fait que le texte relié reste caché au lecteur avant d'être activé. À la seule lecture du texte géniteur, la subversion potentielle du démenti n'est pas apparente.

Ce genre de jeux de cache-cache peut donner lieu à une rhétorique $\mathrm{du}$ "faire-croire ", à une exploitation fallacieuse des présomptions avec lesquelles le lecteur approche le texte numérique. Imaginons que dans l'énoncé "Selon Europe 1, François Fillon a démenti toute volonté de candidater à la présidentielle ", l'activation de l'hyperlien ne provoque ni l'affichage du site d'europel.fr ni d'aucun autre article qui prouve, explique 
ou met en question le démenti, mais d'une photo non datée de l'ancien Premier ministre devant un micro. Cette photo suggérerait au lecteur qu'elle documente l'information du démenti, alors qu'elle prouve seulement que le Premier ministre a parlé, un jour, devant un micro. La feintise fait partie du champ des possibles de la rhétorique de l'hyperlien. Son démasquage figure parmi les objectifs d'une rhétorique du texte numérique.

L'hyperlien met en relation les répertoires et stratégies du texte géniteur et du texte relié de diverses manières qui relèvent de la disposition rhétorique. L'hyperlien est par ailleurs un texte manipulable, qui réunit du texte à lire et des gestes. Certains de ces "énoncés de gestes », comme l'enchaînement d'un appui et d'un relâchement bref et non répétitif, font potentiellement appel à des actions que le lecteur a déjà effectuées ailleurs, en activant un interrupteur. Ce renvoi à un " type " participe à susciter un certain nombre d'attentes sur le résultat de la manipulation, comme je le montrerai à partir d'exemples du corpus journalistique.

\section{FIGURES DE LA LECTURE DU TEXTE NUMÉRIQUE DANS LES DISCOURS INFORMATIF ET ARGUMENTATIF}

\section{Constitution du corpus journalistique}

Beaucoup de journaux et magazines français ont développé ces dernières années des versions numériques pour la lecture écran (lemonde. fr, tempsreel.nouvelobs.com, lexpress.fr...) ; certains titres sont même uniquement accessibles sur Internet (lepost.fr). Face à l'émergence des formats du Web 2.0 incluant les blogs et les réseaux sociaux, la presse en ligne a essayé de s'appuyer sur de nouveaux modèles économiques (par exemple, l'intégration massive d'articles rédigés par des auteurs non professionnels), et de faire émerger de nouveaux services (l'ouverture des articles aux commentaires), mobilisant d'autres outils d'écriture (notamment les systèmes de gestion de contenu). En réaction à ces évolutions importantes, de nombreuses études ont été consacrées aux questions soulevées par le journalisme participatif [Pélissier et al., 2010 ; Rebillard et Touboul, 2010] et aux bouleversements socioéconomiques provoqués par le passage au numérique [Estienne, 2007 ; Dolbeau-Bandin, 2008]. La pratique de l'hyperlien par les journalistes est, surtout à partir de l'année 2000, passée au second plan des recherches consacrées au journalisme en ligne. 
Le potentiel d'action de l'hyperlien s'est pourtant diversifié de façon impressionnante - voilà le constat qui s'est imposé lorsque j'ai exploré, de décembre 2010 à avril 2011, plusieurs centaines d'articles de presse en ligne. Après cette première phase de repérage, j’ai constitué un corpus représentatif de 25 articles $^{2}$, l'article étant défini comme une unité textuelle relativement close et stable, intégrée dans une page-écran composée d'éléments souvent hétéroclites (renvois vers les titres d'actualité, des publicités, un espace commentaires, etc.), et parfois dynamiques. Chacun des articles sélectionnés s'étale sur une seule page-écran.

Le choix des 25 articles du corpus dans lexpress.fr (13 articles), marianne2.fr ( 7 articles) et lepost.fr ( 5 articles) se justifie par une utilisation fréquente de l'hyperlien (au moins trois occurrences, voir p. 237 pour la liste). Les trois médias se présentent non pas comme des «portails d'information » collectant et relayant des dépêches publiées par les agences de presse, mais comme des héritiers d'une presse d'opinion où le discours argumentatif joue un rôle important. Les textes sélectionnés m’ont permis d'examiner le rôle particulier de l'hyperlien dans ce que Philippe Breton [1995] considère comme les « deux registres principaux d'écriture médiatique » : le discours informatif et le discours argumentatif, "deux modèles de représentation du réel ».

Dans le cas de l'information, comme le spécifie Philippe Breton, il faut « un réel supposé objectivable » et un témoin : « La règle, à la fois technique et source d'une déontologie de l'information, va être le maintien de l'intégrité du réel (sous certaines conditions de mise en forme) » (4). Malgré la mise en cause fréquente de l'objectivité de la presse, « toute la crédibilité de l'information médiatique est basée sur le partage de ce contrat », avance l'auteur. Dans le discours informatif se trouve donc préfiguré un lecteur prêt à accepter ce contrat d'objectivabilité et de vérifiabilité.

Le discours argumentatif mobilise, selon Perelman et Olbrechts-Tyteca [1970, 5], des techniques qui permettent de « provoquer ou d'accroître l'adhésion des esprits aux thèses qu'on présente à leur assentiment ». Il préfigure donc un lecteur prêt à recevoir des arguments, à se laisser convaincre tout en reconnaissant qu'il y a plusieurs interprétations possibles du réel.

J'ai écarté de mon corpus les articles s’inscrivant dans une actualité immédiate, brûlante, saisie sur le vif, par exemple les dépêches : l'hyperlien y est souvent absent. L'insertion d'un hyperlien qui dépasse la simple fonction de « renvoi à la source » nécessite de toute évidence une distance

2. Voir le corpus à la fin du chapitre III, p. 237. 
réflexive vis-à-vis des événements relatés. Les articles sélectionnés entre décembre 2010 et avril 2011 font appel aux répertoires les plus divers : des lois de la bioéthique à l'affaire Galliano, de la position de l'Union européenne (UE) face à la migration au succès d'une marque de jean.

Afin de circonscrire de façon rigoureuse les limites de mon projet et d'assurer sa faisabilité, j'ai pris la décision de considérer les 25 articles sélectionnés comme des textes géniteurs, et d'intégrer dans mon analyse seulement les textes reliés au "premier niveau ». Je n'ai pas continué l'exploration des hyperliens implantés dans les textes reliés, bien que ceux-ci deviennent souvent à leur tour des textes géniteurs. Cette décision n'est pas un reflet des pratiques courantes de la lecture numérique. Elle définit un périmètre de lecture possible. D'autres lecteurs choisiraient peut-être d'activer un seul hyperlien dans un texte géniteur, un autre hyperlien dans le texte relié et ainsi de suite, sans jamais revenir vers le texte géniteur. La définition du périmètre de lecture est une décision personnelle, qui peut néanmoins être argumentée. Dans le cadre de cette étude, j'ai opté pour ce périmètre précis afin d'effectuer la lecture la plus exhaustive possible d'un texte géniteur avec tous ses textes reliés.

J'ai commencé l'étude de chaque article par une analyse de la pageécran. Comme les trois médias ont mis en place des systèmes de gestion de contenu pour la publication des textes, les pages-écran à l'intérieur d'un même média se ressemblent beaucoup. Deux des trois médias sélectionnés font, par leur titre, leur logo et certains codes couleur, explicitement référence à un pendant papier qui suscite potentiellement des attentes concernant les répertoires, les stratégies et le potentiel d'action de l'hyperlien. Certaines formes-modèles du texte, par exemple la police des caractères et la mise en forme des hyperliens, et certaines formesmodèles de la page-écran, comme la segmentation de l'article, contribuent également à l'émergence d'attentes. Les spécificités de l'outil d'écriture sont à leur tour porteuses de figurations, soutenant une rhétorique spécifique qui sera prise en compte dans l'analyse.

Pour chaque texte géniteur, j'ai d'abord identifié les répertoires et stratégies sans activer encore les hyperliens. Je me suis en particulier intéressée à l'emplacement de l'hyperlien dans l'énoncé, et aux éventuelles annonces ou reprises qui préparent le terrain au texte relié. En m’appuyant sur les études empiriques citées au chapitre II, j'ai énoncé pour chacun des 229 hyperliens dans les 25 articles du corpus des hypothèses concernant l'horizon d'attente extra-textuel du lecteur. J'ai enfin activé les hyperliens et identifié les répertoires et stratégies de chaque texte relié 
afin d'évaluer son niveau de contiguïté avec le texte géniteur : répond-il aux attentes suscitées par le texte géniteur en fonction de l'horizon intratextuel et extra-textuel, ou met-il plutôt ces attentes au défi ? Et quelles sont les figures de la lecture qui émergent ainsi ?

\section{Formes-modèles de la page-écran et du texte}

Avant de venir aux textes à lire, il me paraît important d'examiner les formes-modèles des pages-écran et du texte. Je rappelle qu'Yves Jeanneret et Emmanuël Souchier [2005] définissent les formes-modèles comme des protocoles de lecture qui contribuent au potentiel d'action d'un texte ; elles constituent les cadres qui bordent et contiennent ce texte sur les pages d'un support de lecture. Dans le domaine du texte numérique, ces formes-modèles font en outre appel à des formats éditoriaux plus anciens, notamment le magazine papier.

\section{marianne2.fr}

Le magazine d'information hebdomadaire Marianne a été fondé en 1997 par Jean-François Kahn et Maurice Szafran. Kahn le définit comme un média " centriste révolutionnaire » souhaitant combattre la "pensée unique ». Par son histoire et les multiples polémiques suscitées, le titre Marianne provoque potentiellement des attentes de prises de position fortes.

Dans les mentions légales du site, marianne2.fr est présenté comme un « journal en ligne édité par la société Marianne SA », et non pas comme le pendant numérique du magazine papier. Le « 2 » dans le nom de l'édition numérique marque également la différence. Le logo est pourtant le même que celui du magazine papier. Le rouge, couleur-phare de Marianne papier, se retrouve dans l'en-tête de chaque page-écran, dans l'encadré des mentions légales en bas, dans les titres et sur les hyperliens.

Chaque article commence par une lettrine. Le texte courant s'affiche dans une police " avec serif » (empattements rapprochant les caractères), et cela bien qu'un consensus se soit établi ces dernières années autour de l'idée qu'il faut privilégier les polices « sans serif » pour la lecture écran : "Les études de lisibilité à l'écran montrent que le texte sans serif est là plus rapide à lire ", affirment par exemple Jakob Nielsen et Hoa Loranger [2007, 232]. Le choix de marianne2.fr pour une police avec empattements peut être interprété comme la volonté de non seulement renvoyer à la 
« respectabilité » de l'écriture papier, mais d'empêcher une lecture hâtive et extensive du texte. La structure des articles de marianne2.fr, découpés en paragraphes relativement longs, constitue un autre élément rappelant le formatage du magazine papier.

Le menu présenté en dessous de l'en-tête (marquée par le logo et la couleur rouge) permet d'accéder aux rubriques du magazine : " économie », " international », " société "... Un onglet spécifique est dédié aux « blogs associés », dans lesquels certains articles sont effectivement puisés par la rédaction professionnelle du magazine. L’accueil d'auteurs amateurs est un trait marquant du journalisme numérique. Dans marianne2.fr, l'identité et le statut de chaque auteur sont clairement spécifiés. Au lieu d'une simple signature est inséré dans chaque article un encadré comportant la photo du journaliste, son nom, sa fonction (par exemple, « rédacteur chez Marianne »), ainsi que ses principaux champs d'expertise. La taille de la photo rappelle potentiellement celle des « images de profil » sur Facebook. L'indication du nombre d'articles publiés peut être interprétée comme une preuve de la confiance accordée à l'auteur par le média.

Un autre encadré inséré dans l'article permet d'activer, par clic sur leurs titres hyperliés, quelques-uns des articles écrits par le même auteur. Le lecteur a aussi la possibilité de suivre l'auteur sur Twitter ou de lui envoyer un mail. Le rôle du journaliste a décidément changé sur Internet. Il ne suffit plus de publier un article ; il faut assurer un suivi, et éventuellement la modération d'un débat.

À droite de l'article s'alignent différents encadrés. Le premier en haut renvoie aux actualités, représentées par des titres qui changent plusieurs fois par jour. Seuls les tags, principaux mots-clés associés à l'article qui s'affichent en dessous du texte, permettent au lecteur d'accéder à d'autres articles traitant de la même thématique.

$\mathrm{Au}$ milieu de quelques encadrés publicitaires, le lecteur repère un encadré marqué par le message suivant : " Dans cette rubrique vous aimez "lire" et "réagir" ». Le mot " lire » est présenté dans un carré rouge, le mot " réagir " dans un carré bleu. La lecture se trouve donc associée à la couleur rouge représentant le magazine Marianne ; à travers le bleu, le "réagir » renvoie à un autre univers, clairement séparé de la partie éditoriale assurée par marianne2.fr : la partie des pratiques tournées vers une intervention du lecteur, celle dans laquelle ses commentaires s'enchaînent. Cette partie n'est pas affichée immédiatement lorsque le lecteur arrive sur la page-écran. Elle est annoncée par un symbole activable en bleu, situé en bas des articles. 
L'hyperlien, marqué en rouge, est associé à l'univers de la lecture. Ce fait est remarquable : comme l'indiquent Yves Jeanneret et Emmanuël Souchier [2005, 7], d'autres figurations de l'hyperlien opposent plutôt la « fluidité » du texte numérique à la rigidité du texte papier, allant parfois jusqu'à associer hyperlien et oralité. En tant qu'icône manipulable, l'hyperlien renvoie souvent à des idées de déclenchement et de réactivité immédiate. Dans marianne2.fr, il reste, par sa forme-modèle, associé à l'univers de la lecture.

Les principaux éléments de la page-écran de marianne2.fr, comme les couleurs, la taille et le positionnement des encadrés, la largeur et la structuration des articles en titre, chapeau et paragraphes avec sous-titres, sont prescrits par un système de gestion de contenu. Dans les mentions
Figure 5. Le haut et le milieu d'une pageécran de marianne2.fr
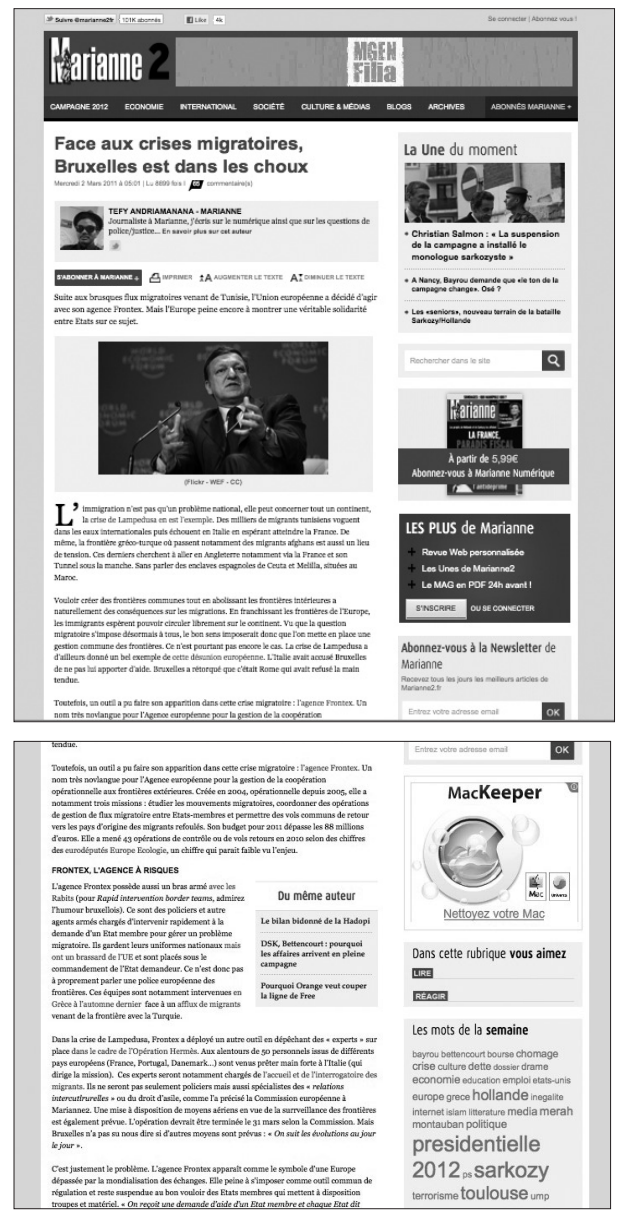
légales accessibles en bas de la fenêtre, le lecteur apprend qu'il s'agit de l'outil Webzine Maker. Cet outil est publiquement accessible sur le site de l'entreprise chargée de son développement. Tout lecteur peut le tester avant d'acquérir éventuellement une version payante. Après avoir accédé à l'interface de saisie, il peut en quelques clics créer un Webzine ressemblant graphiquement à marianne2.fr. La facilité d'accès et de manipulation de l'outil participe à cette désacralisation du rôle du journaliste qui se reflète aussi dans l'ouverture du site aux blogueurs associés. 
Si l'auteur veut créer un hyperlien dans Webzine Maker, il sélectionne le texte à hyperlier et clique sur le bouton « lien ». S'ouvre un menu qui différencie entre le lien externe, pour lequel une adresse http complète doit être saisie, et le lien interne, pour lequel l'outil propose les titres d'articles déjà publiés dans la rubrique. Cette fonctionnalité renvoie à une figuration avant tout informationnelle du texte journalistique, dans laquelle l'article complet confirme l'annonce faite par le titre. Dans les faits, beaucoup de titres de marianne2.fr sont pourtant métaphoriques (voir article $22 \mathrm{du}$ corpus reproduit dans la figure plus haut et analysé en détail plus loin : "Face aux crises migratoires, Bruxelles est dans les choux »).

Il est important de prendre en compte cette divergence entre la présomption informationnelle envers l'hyperlien anticipée par les fonctionnalités de l'outil, et les procédés rhétoriques mobilisés par certains titres d'articles. Les auteurs de marianne2.fr pourraient ainsi être tentés d’insérer un hyperlien à la seule vue du titre proposé par l'outil, sans vérifier si le texte relié s'inscrit vraiment dans une contiguïté logique avec le texte géniteur. L’outil propose à l'auteur une rhétorique préfigurée.

\section{lexpress.fr}

Le magazine L'Express a été fondé en 1953 comme supplément hebdomadaire du journal Les Échos par Françoise Giroud et Jean-Jacques Servan-Schreiber. Premier magazine d'information français, il a accueilli des rédacteurs célèbres : Albert Camus, Jean-Paul Sartre, François Mitterrand, Madeleine Chapsal... Cette liste a certainement contribué au positionnement du magazine dans l'imaginaire des lecteurs. Ils s'attendent potentiellement à des prises de position fortes par des personnalités connues, à des investigations documentées et des analyses de fond.

Le logo rouge de L'Express papier marque pourtant seulement la page « actualités » du site. Dans les autres rubriques, ce logo s'affiche plus discrètement, en gris clair, et se trouve parfois juxtaposé à d'autres visuels : la rubrique "Économie » accueille par exemple, en haut à gauche, le logo jaune de L'Expansion. Le site se présente non pas comme la version en ligne d'un magazine papier, mais comme le portail du groupe Express Roularta.

Comme dans marianne2.fr, les articles sont affichés à gauche sur la page-écran de lexpress.fr. À droite s'alignent des encadrés proposant des services, donnant par leur titre accès à d'autres articles ou à des offres commerciales. Dans la rubrique « Emploi » (voir figure 6), un formulaire 
permet de consulter des offres d'emploi sélectionnées par lexpress.fr. Plusieurs encadrés sur la page y renvoient également.

La forme-modèle graphique des textes, une police sans empattements et plutôt arrondie, répond aux conventions établies ces dernières années dans le webdesign et préfigure un accès rapide aux informations. Les recommandations aux stagiaires de lexpress.fr rédigées et rendues publiques par Éric Mettout [2010], rédacteur en chef, font également appel à cette figuration de la lecture numérique. L'auteur doit, d'après Mettout, structurer ses textes selon le principe de la pyramide renversée : l'essentiel en haut, l'anecdote en bas. Titre, chapeau et sous-titres doivent répondre aux cinq questions " en w » (why, what, who, when, where) afin de faciliter la lecture rapide. Sur la page-écran de lexpress.fr, chaque article est donc en effet précédé d'un avant-titre court qui le situe dans un contexte thématique (voir l'affaire Galliano), et d'un bref chapeau qui est mis en évidence par une taille de caractères plus grande.

Ces figurations d'un lecteur pressé sont certes bien connues de tous les journalistes professionnels, même ceux qui ont appris leur métier dans un journal papier ${ }^{3}$. Sur un dispositif de lecture numérique, où le texte géniteur disparaît au moins temporairement de l'écran après l'activation du texte relié, ces éléments de structuration peuvent pourtant, de façon particulièrement efficace, jouer un rôle d'aide-mémoire et de contextualisation rapide.

Figure 6. Le haut (à gauche) et le milieu (à droite) d'une page-écran de lexpress.fr
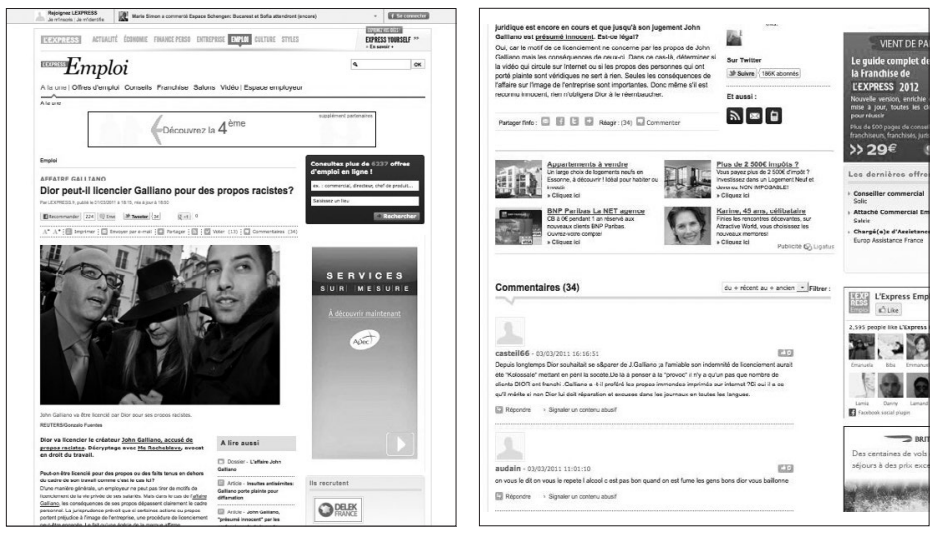

3. Lors de ma formation de journaliste à la Passauer Neuen Presse en Allemagne, j’ai également été confrontée à la « règle des $5 \mathrm{w}$ ». 
Plusieurs onglets sur la page-écran invitent le lecteur à rejoindre l'application « express yourself». Cette application testée depuis novembre 2011 pour la rubrique "Culture », est désormais disponible pour toutes les rubriques du site. Elle donne aux lecteurs de lexpress.fr la possibilité de devenir eux-mêmes auteurs d'un article.

Même si la signature de l'auteur et son statut s'affichent de façon beaucoup plus discrète que dans marianne2.fr, une place importante est donnée aux pratiques d'intervention laissant des traces visibles dans le dispositif. En dehors de l'application " express yourself », plusieurs autres éléments sur la page-écran renvoient à la représentation d'un lecteur actif qui a envie non seulement de lire, mais de faire. Au-dessus et en dessous du texte de l'article s'alignent des boutons incitant au partage et à la « lecture sociale » : l'article peut être recommandé sur Facebook et Google +, commenté sur Twitter et envoyé par mail. Un petit encadré à la fin de l'article propose de suivre lexpress.fr sur les réseaux sociaux et d'apposer un «like ». Dans les encadrés autour du texte géniteur s'affichent non seulement les titres des dernières " actualités », mais aussi « les + des internautes » : les titres des cinq articles les plus lus et commentés. Contrairement à marianne2.fr, où la zone " commentaires » doit d'abord être activée pour que les contributions s'affichent, les commentaires s'alignent dans lexpress.fr directement sur la page-écran. Le petit symbole du pouce Facebook offre même la possibilité d'approuver chaque commentaire.

Le système de gestion de contenu de lexpress.fr s'appelle Ocari et a été développé spécifiquement pour l'entreprise Express Roularta. Sur plusieurs sites Web, il est annoncé comme un outil open source, mais au moment de la rédaction de ce livre, il n'est pas encore mis à disposition du grand public. Un document de présentation par les développeurs Sébastien Angele et Jérôme Macias, accessible sur Internet ${ }^{4}$, permet néanmoins de relever quelques spécificités.

En dehors des champs habituels pour le titre, le sous-titre et le chapeau, l'interface de saisie d'Ocari contient un renvoi vers la « médiathèque » et deux encadrés " flux » répertoriant de façon dynamique les titres des derniers articles publiés dans une rubrique. Comme dans le système de gestion de contenu de marianne2.fr, il est donc plus facile pour le journaliste d'insérer un hyperlien vers un article interne : fonctionnalité qui conforte potentiellement les intérêts commerciaux du média. Comme

4. Sébastien Angele et Jérôme Macias, « Développement du CMS Ocari avec Symfony », Symfony Paris, 11-12 juin 2009, [En ligne] : < http://www.symfony-project.org/get/sflive09fr/symfonylive-ocari.pdf $>$. 
dans le système de gestion de contenu de marianne2.fr, les articles internes sont seulement représentés par les titres. La recommandation d'Éric Mettout [2010] concernant l'annonce des enjeux essentiels du texte dès le titre entre en résonance avec cette fonctionnalité.

Dans le backoffice de l'application " express yourself » en revanche, réservé aux auteurs non professionnels, aucune différenciation n'est faite entre liens externes et liens internes. Un encadré à côté des champs de saisie permet néanmoins de suivre les "dernières actualités » de la rubrique par affichage des titres, et incite potentiellement à les relier en priorité au texte géniteur. Les hyperliens apparaissent ensuite sur le frontoffice marqués par un soulignement - convention graphique présupposée connue par un lecteur habitué aux pages-écran numériques.

\section{lepost.fr}

Lepost.fr a été fondé en septembre 2007 comme une émanation du Monde interactif. En janvier 2012, l'actualisation du post.fr a été arrêtée. La page d'accueil redirige désormais vers le site du Huffington Post. Chaque article de l'ancien site est maintenant entouré du logo et des titres d'actualité de ce magazine.

Lepost.fr a été conçu comme un site d'actualité collaboratif, alimenté à la fois par des journalistes salariés et par des auteurs-amateurs. Après inscription, tout lecteur pouvait laisser un post sur le serveur ; s'il était sélectionné par la rédaction, ce post (qui avait bien la forme d'un article) pouvait remonter sur la une. À plusieurs égards, lepost.fr a préparé le terrain à un journalisme intégrant de façon massive des textes rédigés par des rédacteurs non professionnels. Lepost.fr est le seul, parmi les trois médias sélectionnés, à ne pas s'appuyer sur une édition papier. Toutes les polices sont sans empattements et renvoient potentiellement à la figuration d'une lecture-écran allant rapidement à l'essentiel de l'information.

Tout en bas de la page-écran s'affiche le descriptif de la ligne éditoriale : lepost.fr « combine la rigueur d'un journal en ligne et la liberté de ton d'un blog ». Ne serait-ce qu'à travers son nom, le post fait référence aux textes publiés sur les réseaux sociaux et les blogs, et oriente potentiellement les attentes du lecteur vers la lecture de billets d'humeur et autres prises de position fortes. Des formes-modèles caractéristiques du blog se retrouvent à plusieurs endroits de la page-écran. En dehors de la hiérarchisation des articles et de l'affichage direct des commentaires, c'est la forte présence des tags (mots-clés hyperliés indiquant les principaux thèmes de l'article 
et renvoyant vers d'autres articles marqués par les mêmes mots-clés) qui évoque l'univers de la textualité numérique. Une liste de tags est également présente sur les pages-écran de marianne2.fr, mais seul lepost.fr met en avant un tag principal en haut de chaque article qui précède le titre principal.

Alors que les tags s'affichent en bleu plutôt clair, les marqueurs de l'hyperlien se différencient du texte courant en noir par une couleur bleu sombre. Les titres d'autres articles renvoyant vers des sujets connexes, alignés à la fin du texte géniteur et dans la marge droite de la page-écran, sont affectés de la même couleur bleu sombre. En revanche, ces titres renvoient exclusivement à des articles internes, alors que les hyperliens intégrés dans le texte géniteur mènent fréquemment à des textes extérieurs au site du post.fr (voir statistiques plus loin).

La facilité d'accès et de manipulation de l'outil d'écriture se reflète dans la structuration sobre de la page-écran, et renvoie à l'imaginaire d'un journal désormais rédigé par tous, journalistes professionnels et amateurs. Un lecteur adhérant à la figuration d'un espace public marqué par une liberté d'expression généralisée, et d'un journalisme où les opinions se croisent et se juxtaposent librement, approuve potentiellement ces formes-modèles.

Les attentes d'un lecteur qui réserve sa confiance plutôt à une presse reconnue comme professionnelle pourraient en revanche être mises au défi. En effet, l'histoire courte de ce média a été marquée par plusieurs scandales, dont l'annonce erronée de la mort d'une personnalité publique par un rédacteur amateur. Un lecteur informé de ces dérives approche peut-être les textes géniteurs et les hyperliens intégrés à ces textes avec prudence, s'attendant potentiellement non pas seulement à un dialogisme fort, mais à un jeu frauduleux avec ses expectatives.

Figure 7. Le haut (à gauche) et le milieu (à droite) d'une page-écran du post.fr
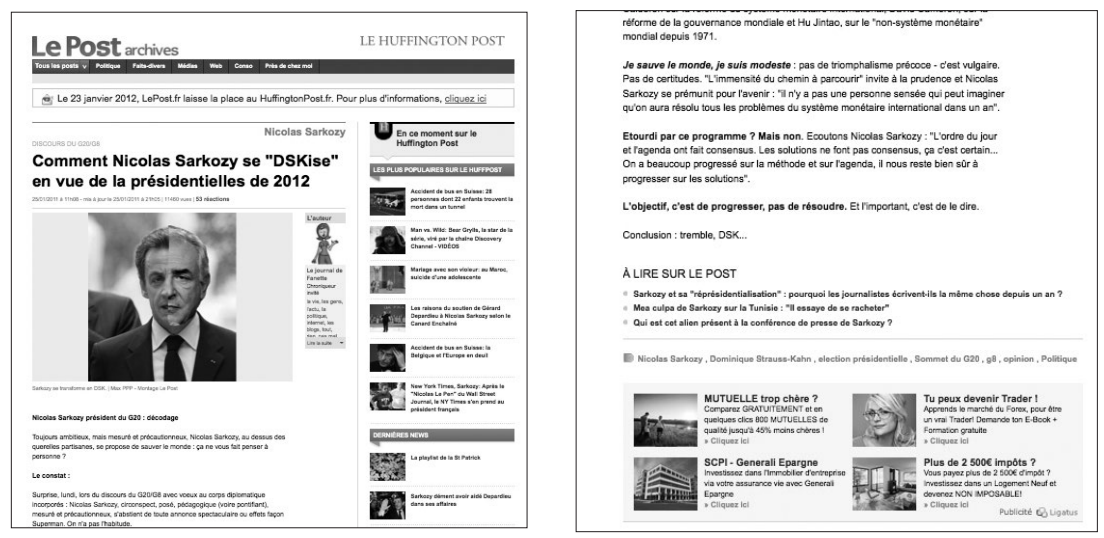
Les commentaires des lecteurs s'alignent en dessous des articles. À côté de l'onglet « commentaires », un deuxième onglet permet d'accéder aux posts publiés en réaction au texte géniteur : contrairement à marianne2.fr qui distingue clairement entre « lire » et « réagir », lepost. fr recourt ainsi très explicitement à la figuration d'une lecture fondée sur la fusion entre pratiques de lecture et d'écriture. La couleur rose mettant en évidence à la fois le logo du post.fr, le nombre de commentaires et les commentaires eux-mêmes ainsi que le nom de l'auteur, crée une cohérence visuelle entre ces éléments et peut être considérée comme la trace d'une volonté de souligner l'invitation à ces pratiques.

Chaque lecteur inscrit au post.fr est représenté par une image de profil. L'activation des images permet d'accéder aux «blogs associés ». Ces blogs sont caractérisés globalement par les mêmes formes-modèles que le site principal : autre élément effaçant les frontières entre participations professionnelles et amateurs, lecteurs et auteurs.

La signature de l'article est, comme dans marianne2.fr, assez élaborée : dans un petit encadré s'affiche l'image de profil de l'auteur, le titre de son blog et sa fonction. En quelques mots, l'auteur résume ses centres d'intérêt et ses domaines d'expertise. L'inscription à l'outil blog étant désormais désactivée, il n'est plus possible de vérifier si l'outil favorisait éventuellement le lien interne, comme c'est le cas pour marianne2.fr et pour lexpress.fr. Comme marianne2.fr et lexpress.fr, lepost.fr a créé son propre réseau social. Il est cependant le seul parmi les trois médias étudiés à ne pas renvoyer vers des réseaux comme Facebook ou Twitter.

\section{Formes-modèles anticipant sur des pratiques de lecture}

L'analyse des pages-écran sélectionnées a fait émerger trois formesmodèles de la page-écran et du texte particulièrement prégnantes. Je précise encore une fois qu'il s'agit là de formes-modèles qu'on peut analyser avant d'entreprendre la lecture d'un article précis.

\section{Les formes-modèles « pro-intensives »}

Certaines formes-modèles anticipent sur des pratiques de lecture plutôt lentes et concentrées, que je propose d'appeler " pro-intensives ». La police avec empattements dans marianne2.fr peut être considérée comme l'une de ces formes-modèles. Renvoyant aux caractères traditionnellement 
utilisés dans la presse papier, cette police ne fait pas que doter les articles d'une certaine respectabilité ; elle constitue un obstacle possible pour une lecture voulant passer trop vite sur les contenus.

L'imitation de certaines caractéristiques héritées du magazine papier, comme une longueur importante des articles et paragraphes, peut créer une familiarité rassurante [voir Doueihi, 2011, 12] et renvoyer à l'imaginaire d'une certaine déontologie professionnelle ; elle préfigure un lecteur prêt à prendre son temps et à se plonger dans des structures argumentatives parfois complexes.

Comme pour souligner la revendication d'une lecture concentrée et intensive du texte numérique, marianne2.fr associe les hyperliens par leur couleur rouge à l'univers du " lire », alors que l'espace " commentaires » est associé par la couleur bleue à l'univers du « réagir ».

\section{Les formes-modèles « pro-interventives »}

Les formes-modèles pro-intensives sont, dans les trois médias, contrebalancées par des formes-modèles " pro-interventives » qui renvoient à des formats numériques modifiables comme le blog : l'espace « commentaires ", la possibilité d'accéder au backoffice du média pour proposer soi-même un article, et les invitations multiples à la participation active du lecteur via le partage avec son réseau social, préfigurent des pratiques où le sujet, loin de laisser seulement une trace de son activité de lecture, manifeste son existence en affichant son rapport aux autres.

Elles reflètent aussi un profond changement du statut de l'auteur-journaliste, des figurations du rôle de la presse et du texte lui-même, conçu non plus comme un produit stable et fixe, mais comme un réseau textuel proliférant, constamment modifiable et partageable, qui met en branle la hiérarchie traditionnelle entre auteur et lecteur.

Alors que les formes-modèles liées à la couleur et à la structuration de la page-écran de marianne2.fr suggèrent une séparation claire entre " lire » et " réagir », lepost.fr répond pleinement à la figuration d'une « lecture interventive » où activités de lecture et d'écriture se confondent. Les commentaires des lecteurs s'alignent directement en dessous de chaque article sans devoir être activés.

Dans lexpress.fr, une multiplicité d'éléments graphiques invite également au partage immédiat des contenus sur les réseaux sociaux et rentre donc également dans la catégorie des formes-modèles pro-interventives. 
Les formes-modèles « pro-extensives »

Un autre type de forme-modèle encourage potentiellement des pratiques de lecture rapides et impatientes, qui sont souvent spontanément associées aux formats numériques (voir chapitre II). Je propose d'appeler ces formes-modèles « pro-extensives ». Pour lexpress.fr, le rédacteur en chef recommande explicitement une structuration des articles répondant au désir du lecteur d'accéder rapidement aux informations du texte : un avant-titre, un titre et un chapeau courts doivent donner des renseignements clairs sur le contenu du texte ; chaque article doit être segmenté en brefs paragraphes précédés de sous-titres.

La police des textes courants dans lexpress.fr, sans serif et plutôt arrondie, est un autre élément formel que je propose d'appeler " proextensif ». Les formes-modèles anticipant sur la nature iconique des hyperliens peuvent également encourager des pratiques de lecture extensives. Plus globalement, le haut niveau de tabularité des pages-écran de lexpress.fr peut rencontrer les attentes d'un lecteur pressé, à la recherche d'une information précise.

\section{Tableau 1. Formes-modèles de la page-écran et du texte}

\begin{tabular}{l|l}
$\begin{array}{l}\text { Formes-modèles } \\
\text { pro-intensives } \\
\text { préfigurent des } \\
\text { pratiques plutôt } \\
\text { concentrées et lentes. }\end{array}$ & $\begin{array}{l}\text { Police avec empattements ; relative longueur } \\
\text { des articles et paragraphes ; structuration } \\
\text { des articles rappelant un pendant papier. }\end{array}$ \\
$\begin{array}{l}\text { Formes-modèles } \\
\text { pro-interventives } \\
\text { préfigurent un lecteur } \\
\text { souhaitant laisser des } \\
\text { traces dans le texte. }\end{array}$ & $\begin{array}{l}\text { Espace « commentaires » et autres invitations } \\
\text { à la participation active du lecteur, possibilité } \\
\text { d'accéder au backoffice renvoyant à des formats } \\
\text { numériques modifiables comme le blog ; symboles } \\
\text { Formes-modèles } \\
\text { pro-extensives } \\
\text { préfigurent un lecteur au partage sur les réseaux sociaux. } \\
\text { impatient, souhaitant } \\
\text { aller vite dans } \\
\text { l'exploration du texte. }\end{array}$ \\
\hline
\end{tabular}$\quad \begin{aligned} & \text { Structuration des articles mettant en évidence } \\
& \text { les informations principales du texte ; encadrés } \\
& \text { synthétiques hyperliés ; police sans empattements ; } \\
& \text { haut niveau de tabularité de la page-écran ; } \\
& \text { marquage des hyperliens incitant à la navigation. }\end{aligned}$

Après l'étude des formes-modèles qui marquent et cadrent les textes sur la page-écran, il s'agit maintenant de passer à l'étude du niveau de contiguïté entre les textes géniteurs et les textes reliés par hyperliens, 
ainsi que du couplage entre le texte à lire et les gestes de manipulation, et d’identifier les figures de la lecture de l'hyperlien qui en émergent.

\section{Figures de la lecture de I'hyperlien}

Comme le laissent penser les études empiriques citées au chapitre II, beaucoup de lecteurs approchent l'hyperlien avec l'idée d'accéder à des éléments factuels, des compléments d'information, explications, définitions, preuves et illustrations. Une deuxième figuration de l'hyperlien, moins répandue, associe celui-ci à l'idée d'un croisement dialogique de points de vue. L'étude des relations de contiguïté entre texte géniteur et texte relié, anticipées dans les articles du corpus, fait émerger un champ des possibles plus diversifié. Certaines figures de la lecture de l'hyperlien confirment potentiellement les figurations informationnelles ou dialogiques de l'hyperlien, alors que d'autres mettent plutôt au défi ces deux normes d'attente.

À chaque fois que suffisamment d'éléments me paraissent réunis, je propose, dans les pages qui suivent, une interprétation possible des lieux d'indétermination qui s'ouvrent lorsque les expectatives du lecteur sont mises au défi. En m’appuyant sur les trois « temps de l'interprétation » identifiés par Gilles Deleuze [1982] (la « soustraction », la « superposition d'hypothèses » et l'« intégration », voir plus haut), je préciserai quels éléments j'ai sélectionnés dans les textes proposés, et pourquoi je les considère comme pertinents pour l'interprétation. Je testerai parfois différentes hypothèses avant de retenir celle qui me paraît la plus adaptée. Lors de cette dernière étape, l'objet de pensée se trouvera donc finalement ressaisi, stabilisé par les éléments sélectionnés et mis en perspective avec mes propres imaginaires. Toujours incomplète, chacune de mes interprétations constitue avant tout une invitation adressée au lecteur à se lancer à son tour dans une interprétation.

Le relevé des figures de la lecture de l'hyperlien sera accompagné de propositions terminologiques. J'espère que ces mots compacts permettront aux lecteurs, chercheurs et auteurs de situer plus facilement leurs propres expériences. L'objectif de la rhétorique a toujours été de proposer des repères en passant par des typologies ; bien évidemment, ces repères bougeront avec l'évolution de l'horizon d'attente du lecteur et l'élargissement constant du champ des possibles de l'écriture numérique. 
Figures de la lecture « informationnelle»

« Je vérifie sur Internet » : en prononçant cette phrase, le lecteur investit les données disponibles sur le Web de l'espoir d'y trouver des certitudes. Plusieurs études empiriques montrent que les lecteurs approchent fréquemment l'hyperlien avec une présomption d'information. Le texte relié est considéré comme une donnée factuelle que l'on consulte, censée confirmer les attentes suscitées par les annonces et l'emplacement de l'hyperlien dans le texte géniteur. Comme l'exprime Tosca Pajares [2000], ces hyperliens constituent des points de départ « bien marqués » vers des « destinations prévisibles ». Un mot hyperlié « vaut pour » le texte auquel il renvoie [voir Manovich, 2001, 228] et s'inscrit dans une « culture de l'évidence ».

Le contexte de réception journalistique peut renforcer la présomption d'information face à l'hyperlien. Comme l'a constaté Tom Wolfe en 1973, et comme des études empiriques récentes le confirment, le texte journalistique est fréquemment associé à des figurations d'objectivité, de neutralité et de vérifiabilité. D’un web-journaliste, les lecteurs attendent donc potentiellement qu'il fournisse, via hyperliens, des preuves, un accès aux sources utilisées, des compléments d'information factuels. Au lieu d'ouvrir vers un réseau d'associations divergentes, l'hyperlien est censé apporter une délimitation.

Toute écriture opère un tri et une hiérarchisation des données. En tant que procédé rhétorique, la relation de contiguïté matérialisée par un hyperlien est déployée pour interpréter le réel, et constitue une trace de cette interprétation. Une définition neutre n'existe pourtant pas davantage qu'une dépêche objective ; un hyperlien informationnel reliant le récit d'un fait divers à une source ne peut que réduire l'incertitude sur le monde, et non pas la gommer.

Le potentiel d'action d'un hyperlien sera donc appelé informationnel, non pas en fonction de la vérité ou de la vérifiabilité des répertoires du texte géniteur et du texte relié, mais en fonction des annonces faites dans le texte géniteur, et de la confirmation de ces annonces dans le texte relié ; en fonction des attentes suscitées par l'emplacement de l'hyperlien, et de la réponse donnée à ces attentes ; en fonction de la contiguïté thématique, logique ou temporelle entre répertoires et stratégies du texte géniteur et du texte relié ; et en fonction des figurations associées au genre textuel, au média et au support de lecture.

L'hyperlien informationnel préfigure donc un lecteur à la recherche de confirmations, de définitions, d'illustrations, de preuves ou d'explications. 
Il aurait plutôt tendance à encourager des pratiques de lecture extensives, qui sont également préfigurées par certaines formes-modèles de la pageécran : le lecteur peut passer rapidement par-dessus un texte relié qui ne fait que confirmer ce que le texte géniteur a déjà annoncé ; la non-activation du texte relié ne changerait pas fondamentalement l'interprétation du réel déjà proposée par le texte géniteur.

\section{Hyperlien « définissant "}

Dans l'hyperlien « définissant », premier procédé dans la catégorie des figures de lecture informationnelles, le texte relié propose la définition d'un terme technique, d'un dispositif juridique, d'une institution... C'est dans ce cas souvent des mots plutôt rares dans le langage courant, ou des notions complexes, qui se trouvent affectés d'un hyperlien : l'auteur anticipe sur la volonté du lecteur d'en savoir plus sur ces mots et notions. Par le recours à des encyclopédies, des ouvrages de référence et autres « paroles d'experts », l'hyperlien définissant promet de réduire l'incertitude du lecteur, et constitue la trace d'une confiance accordée par l'Auteur à la référence convoquée. On pourrait aller jusqu'à considérer la convocation de certaines références (Wikipédia) comme la trace des pratiques informationnelles du journaliste.

L'article (13) du corpus, prélevé dans marianne2.fr, revient sur l'affaire Pornic : le meurtre d'une jeune fille par un multirécidiviste. Dans le texte géniteur, le lecteur apprend que le directeur interrégional des services pénitentiaires en charge de suivre le "présumé coupable " a été limogé, alors que les magistrats et policiers impliqués ne seront pas sanctionnés. Le journaliste argumente que cette affaire révèle des failles générales dans la collaboration entre policiers et gendarmes. Dans ce contexte sont cités trois dispositifs pour le repérage et le suivi de criminels multirécidivistes : «En octobre 2006 a été lancé le fichier Ariane censé mettre en commun les fichiers Stic des policiers et Judex des gendarmes qui traitent chacun des renseignements autour des auteurs d'infractions » (voir aussi capture d'écran figure 8). L'activation de ces trois hyperliens déclenche l'affichage de définitions dans Wikipédia. Les textes reliés répondent donc potentiellement aux attentes d'un lecteur qui souhaite accéder à des définitions concises de ces termes - à condition qu'il reconnaisse à Wikipédia le statut d'une encyclopédie, et que la convocation de Wikipédia par un journaliste professionnel ne le déconcerte pas. 
Figure 8. Exemple d'un hyperlien potentiellement « informationnel » dans l'article (13) du corpus, qui rentre dans la catégorie de l’hypertexte « définissant »

Mais le ministère oublie un point : la mise en commun des fichiers informatiques. En octobre 2006 a été lancé le fichier Ariane censé mettre en commun les fichiers Stic des policiers et Judex des gendarmes qui traitent chacun des renseignements autour des auteurs d'infractions. Durant les enquêtes Meilhon, policiers et gendarmes ont bien évidemment cherché des informations sur leurs fichiers respectifs... mais sans pouvoir recouper leurs informations. Depuis cinq ans et demi, Ariane est resté en phase de test. Il était censé être pleinement opérationnel en mai 2008

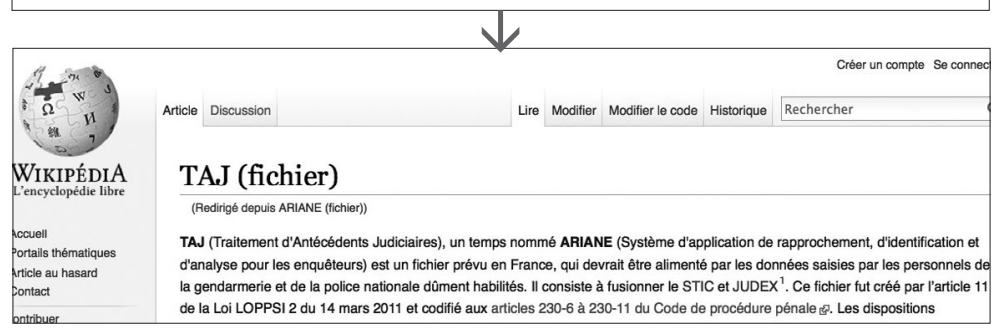

L'activation de l'hyperlien sur « fichier Ariane » provoque l'affichage de la définition du dispositif par Wikipédia.

Il n'y a pas lieu ici de discuter de la fiabilité de cette référence, mais d'évaluer jusqu'à quel point elle peut répondre à la présomption d'information du lecteur. Dans le cadre de l'étude exploratoire menée à l'université Paris 8 (voir plus haut), plusieurs sujets interrogés ont en effet cité Wikipédia comme référence attendue pour la définition d'un terme technique, ce qui montre à quel point cette encyclopédie collaborative est investie d'une valeur informationnelle (dans le sens de réduction des incertitudes). Il est vrai que les formes-modèles convoquées par l'énonciation éditoriale sur la page-écran, ainsi que le nom de Wikipédia luimême peuvent mobiliser des figurations traditionnellement associées à l'encyclopédie papier rédigée par des experts reconnus.

\section{Hyperlien « renvoyant à la source »}

Par un hyperlien « renvoyant à la source », le journaliste relie le texte géniteur à la source qui lui a permis de circonscrire les contours d'un événement. Même si un événement ne peut jamais être décrit et résumé de façon neutre, et même si une information n'est donc jamais objective, des médias telle l'Agence France Presse (AFP) se présentent comme étant « à la source de l'événement », insistant sur la vérification professionnelle 
de chaque information. L'évocation de l'AFP suscite donc potentiellement des attentes de neutralité, d'objectivité et de fiabilité chez le lecteur. Qu'un journaliste professionnel fasse appel à cette source ne surprend sans doute guère ; la possibilité d'accéder à la source rassure potentiellement le lecteur sur le caractère fiable des informations avancées. L'hyperlien renvoyant à la source peut rappeler la note de bas de page dans l'univers papier, à la différence (capitale) près que le texte relié est la source.

Les attentes du lecteur sont orientées encore plus fortement vers la découverte de la source d'information, si cette source est citée nominativement dans le texte géniteur. Dans l'article (20) consacré au remaniement du gouvernement en février 2011, le journaliste de lexpress.fr fait explicitement référence à un autre média : "Selon Europe 1, l'ex-ministre de l'Intérieur ne s'attendait pas à être sorti avant dimanche matin ». Le texte relié, publié sur europe1.fr, cite en effet plusieurs témoins, dont « un ministre » qui confirme cette affirmation, et peut satisfaire la présomption d'information du lecteur. Le renvoi vers le média ayant publié l'information en premier libère aussi l'auteur d'une part de sa responsabilité. Comme le souligne Antoine Compagnon [1979, 40], la convocation d'un autre texte permet à l'auteur de s'y " miroiter »; en même temps, l'auteur désigne son emprunt du doigt et se disculpe.

\section{Hyperlien « illustratif »}

Profitant du fait qu'il est facile et peu coûteux d'insérer des images et des vidéos dans les pages-écran, certains journalistes relient le texte géniteur à des illustrations. Je propose donc de parler d'un hyperlien « illustratif ", qui préfigure un lecteur souhaitant accéder à une confirmation visuelle des informations avancées dans le texte géniteur.

Dans l'article (22) de marianne2.fr consacré aux difficultés de l'UE pour gérer les flux migratoires de façon coordonnée, le journaliste explique que les soldats de Frontex portent l'uniforme de leur pays d'origine, « mais ont un brassard de l'UE ». La photo reliée montre un soldat de Frontex avec son brassard, et répond potentiellement aux attentes d'un lecteur en quête de confirmations visuelles de l'information.

\section{Hyperlien « fournissant des preuves »}

Proche de l'hyperlien renvoyant à la source d'une information, proche aussi de l'hyperlien illustratif, l'hyperlien « fournissant des preuves » 
confirme les affirmations avancées par le texte géniteur. Pour répondre aux présomptions d'information du lecteur, la présentation des preuves dans le texte relié doit être la plus factuelle et la plus objective possible.

L'article (12) publié par lepost.fr est consacré aux choix des participants pour l'émission de TF1 intitulée Parole de Français. «En 2010, Parole de Français avait été suivie par 8,8 millions de téléspectateurs ", rappelle le journaliste. L'hyperlien mène vers une présentation plus précise de ces chiffres avec indication des sources, et peut satisfaire les attentes d'un lecteur à la recherche de preuves que ce sondage a bien eu lieu et qu'il a donné les résultats évoqués.

Dans l'article (5) de lexpress.fr qui, suite au crime Pornic, énumère et discute les propositions de loi sur la récidive, le journaliste cite un député UMP : " Pour Leonetti, comme pour le chef de file des députés UMP Christian Jacob, des propositions sur l'application des peines peuvent intégrer le futur projet de loi ». L'hyperlien permet de consulter l'annuaire de l'Assemblée nationale, et confirme les fonctions du député citées auparavant dans le texte géniteur. Un tel renvoi permettant de se faire une image de la personne évoquée, rappelle éventuellement les encadrés dans les magazines papier qui présentent une personnalité en quelques mots et chiffres clés ; sauf que les contenus ne sont pas immédiatement visibles sur la page-écran, mais doivent être activés par le lecteur. Dans certains cas, ces résumés de profils peuvent jouer un rôle d'« argument d'autorité ».

\section{Hyperlien « d'autorité »}

En recourant à des personnalités ou des institutions jouissant d'une position d'autorité dans l'espace public, l'Auteur dote potentiellement le texte géniteur d'une crédibilité supplémentaire. Souvent, le nom de cette " autorité » est explicitement évoqué dans le texte géniteur. Il arrive aussi que des personnes acquièrent occasionnellement le statut d'autorité parce qu'ils ont, par exemple, assisté à un événement en tant que témoins. Un hyperlien " d'autorité » préfigure ainsi un lecteur qui se laisse rassurer par la parole d'expert.

Dans l'article (17) de marianne2.fr, le journaliste avance que l'insécurité a augmenté dans les hôpitaux. Pour donner du fondement à cet argument, il renvoie au rapport annuel de l'Observatoire national de la violence hospitalière $(\mathrm{ONVH})$, autorité reconnue dans le domaine médical, qui confirme l'aggravation de la situation : " Pour connaître l'ampleur du phénomène, il faut se pencher sur le dernier rapport annuel de l'ONVH, créé en 2005, et 
qui dépend du ministère de la Santé ». L'activation de l'hyperlien déclenche effectivement l'affichage du site de l'organisme, et donne la possibilité de consulter le rapport. Les informations avancées dans le texte géniteur se trouvent donc non seulement confirmées, mais sont dotées d'une aura d'incontestabilité grâce à la respectabilité de cet organisme.

Dans le corpus journalistique étudié, l'auteur d'un texte relié n'est d'ailleurs jamais indiqué nominativement dans le texte géniteur - à une exception près. Dans l'article (20) de lexpress.fr, le lecteur est incité par hyperlien à "Lire l'analyse de Christophe Barbier ». Christophe Barbier est le directeur de la rédaction de L'Express. L'activation de l'hyperlien déclenche en effet l'affichage de l'article rédigé par Christophe Barbier, qui est ainsi reconnu comme " autorité » par l'auteur du texte géniteur en se trouvant explicitement convoqué. Cet exemple montre la fonction sociale de certains hyperliens (voir aussi réflexions plus loin).

\section{Hyperlien « explicatif »}

L'hyperlien " explicatif » est particulièrement fréquent dans le corpus (voir statistiques infra). Son potentiel d'action peut répondre aux attentes de lecteurs qui cherchent des compléments d'information sans vouloir être déstabilisés par des points de vue divergents ou des contre-arguments. Le texte relié décrit les circonstances d'un événement, explique un comportement ou une décision en avançant des données factuelles. Dépassant le potentiel d'action d'une définition ou d'une illustration, il enrichit la lecture du texte géniteur et entre avec lui dans une forte contiguité causale.

L'article (14) publié dans lexpress.fr est consacré aux difficultés de Nicolas Sarkozy concernant sa politique internationale. L'affaire Michèle Alliot-Marie et les révoltes dans le monde arabe l'avaient contraint à un repli sur la politique nationale. "Lorsque Nicolas Sarkozy a reçu les ministres de l'Économie des pays les plus riches », l'attention de la presse s'est concentrée sur Dominique Strauss-Kahn, avance l'article. Le texte relié permet au lecteur de prendre connaissance d'un résumé des contenus de la réunion publié par l'Agence France Presse. Ce résumé confirme la tenue de la réunion et fournit des compléments d'information sur son contenu. La stratégie de présentation de la dépêche peut satisfaire les attentes d'un lecteur voulant s'informer de façon rapide et concise sur cette réunion.

Dans l'exemple suivant, une lecture plus attentive est anticipée par la relation entre texte géniteur et texte relié. L'article (9) dans marianne2.fr est consacré aux hésitations de Nicolas Hulot concernant sa candidature 
aux présidentielles de 2012. "Le très hésitant Monsieur Hulot "consulte" » : c'est avec un hyperlien que commence cet article, précédé d'un court chapeau introductif. Le positionnement de l'hyperlien dans l'énoncé suscite potentiellement la question de savoir, par activation de l'hyperlien, pourquoi Nicolas Hulot est qualifié de " très hésitant ». Le texte relié fournit un relevé des multiples hésitations de Nicolas Hulot. Ces exemples illustrent l'emploi de l'adjectif «très hésitant » dans le texte géniteur tout en dressant un portrait psychologique de Hulot : portrait qui va au-delà de l'événement spécifique d'une éventuelle candidature à la présidentielle.

Dans l'article (5) de lexpress.fr consacré à l'affaire Pornic, l'auteur explique que plusieurs textes de loi ont été votés ces dernières années suite à des faits divers : "Le dernier en date, qui punit notamment par la prison le refus ou l'arrêt du traitement antihormonal - ou castration chimique -, date de moins d'un an et faisait suite au meurtre et au viol d'une joggeuse par un récidiviste dans l'Essonne ». Le texte relié fournit des explications approfondies sur cette méthode. Dépassant le périmètre d'une définition médicale, il expose ses buts, aboutissements et dangers.

\section{Figures de la lecture « dialogique »}

$\mathrm{Au}$ lieu de confirmer les répertoires et stratégies du texte géniteur, certains hyperliens préfigurent un lecteur prêt à accepter la juxtaposition d'arguments contradictoires, de points de vue divergents, de perspectives changeantes et de focus déplacés. Alors que les textes reliés par un hyperlien définissant ou illustratif peuvent en principe se parcourir rapidement parce que les répertoires du texte relié ne déstabilisent pas l'interprétation du réel proposé par le texte géniteur, les procédés de l'hyperlien " dialogique » invitent le lecteur à effectuer des mises en perspective et des comparaisons attentives, pour lesquelles plusieurs allers-retours entre texte géniteur et texte relié peuvent s'avérer nécessaires.

Certains hyperliens mettent donc en relation arguments et contrearguments, déplacent le focus du texte géniteur ou engagent un changement de perspective. Je propose de nommer « dialogique » cette relation en référence à Mikhail Bakhtine [1929], suivant son idée que le texte se construit dans l'interrelation dialogale par une confrontation de points de vue engagée par le lecteur. La question de savoir si ces figures de la lecture dialogique mettent au défi les attentes du lecteur, dépend des annonces faites dans le texte géniteur, de l'emplacement de l'hyperlien 
sur des mots précis, ainsi que des figurations associées par le lecteur à un genre textuel et à un média.

Je rappelle l'exemple de l'hyperlien posé sur l'énoncé « présumé innocent » dans l'article (21) de lexpress.fr consacré au licenciement du créateur John Galliano. Dans l'étude exploratoire menée à l'université Paris 8 (voir chapitre II), certains étudiants ont affirmé s'attendre à des prises de position fortes, voire à une juxtaposition d'arguments et de contrearguments dans le texte relié : l'énoncé « présumé innocent » est en effet lui-même porteur d'un potentiel d'action dialogique.

Par ailleurs, beaucoup de lecteurs valorisent le rôle de la presse comme organe d'un pluralisme d'idées et de la liberté d'expression. Dans cette figuration, le média se trouve investi de l'idée de confronter des points de vue, et de les soumettre à discussion dans l'espace public. Un tel hyperlien peut mettre en branle la « prétention de vérité » caractéristique d'un journalisme plus traditionnel, comme l'expriment Huesca et Dervin [2003, 282]. L'émergence du paradigme de la "lecture sociale » contribuera peut-être à renforcer cet imaginaire qui, loin de l'idée qu'on « vérifie » des informations sur Internet, associe le texte numérique à la représentation d'un espace public ouvert, fondé sur une « libération des subjectivités » [Cardon, 2009].

Comment les procédés de l'hyperlien dialogique répondent-ils précisément à ces attentes ? Autrement dit, quels sont ses atouts en comparaison avec d'autres stratégies, traditionnellement mobilisées pour juxtaposer des points de vue?

Contrairement à un tableau synoptique qui présente différents points de vue de façon concomitante, l'hyperlien peut d'abord cacher son jeu : son potentiel d'action ne s'actualise que si le lecteur clique. Dans le corpus journalistique étudié, le potentiel dialogique de l'hyperlien se trouve rarement annoncé de façon explicite. Il est ainsi tout à fait possible que le lecteur passe à côté des contre-arguments ou des déplacements de focus, qu'il ne soupçonne même pas leur existence à la seule lecture du texte géniteur.

Une fois activés, des contre-arguments et autres révélations dans le texte relié peuvent fortement ébranler l'interprétation du réel proposée par le texte géniteur ; si aucune annonce de cet ébranlement n'est faite dans le texte géniteur, le lecteur peut certes considérer que l'Auteur donne la préférence aux arguments avancés dans le texte géniteur, et qu'il propose les contre-arguments seulement par acquit de conscience : après tout, c'est d'abord le texte géniteur qui s'affiche à l'écran, et les hyperliens peuvent très bien ne pas être activés. Néanmoins, la lecture de certains 
textes reliés contenant des contre-arguments peut tellement bouleverser la réception du texte géniteur que le lecteur doit se poser la question de leur justification : ces hyperliens constituent-ils la trace d'une volonté de l'auteur de miner son propre texte ? Sont-ils la trace d'une tentative de résistance aux prescriptions éditoriales, à la ligne politique d'un média ? Reflètent-ils une hésitation profonde de l'auteur ? Ou constituent-ils la marque d'intervention d'un autre auteur qui a hypertextualisé après coup le texte d'un collègue pour ouvrir le débat (dans certains médias en ligne, les auteurs des textes géniteurs et des hyperliens ne sont pas les mêmes) ?

Pour le lecteur, une figure de la lecture dialogique peut à la fois constituer une source de trouble, et une opportunité. D'une part, elle ne réduit pas forcément l'incertitude du lecteur sur le monde, et peut mettre au défi sa présomption d'information face à au texte numérique. D'autre part, si le lecteur accepte de mettre en perspective arguments et contre-arguments, il pourra profiter de la possibilité que ces hyperliens lui donnent : celle de se forger sa propre opinion à partir des points de vue exposés. Les figures de la lecture dialogique pourraient ainsi constituer des procédés rhétoriques particulièrement intéressants pour l'édition scientifique.

\section{Hyperlien « déplaçant le focus »}

Certains textes reliés déplacent potentiellement le focus du texte géniteur en ciblant un aspect particulier de la problématique générale ou en présentant un point de vue connexe. Même s'il ne va pas jusqu'à mobiliser des contre-arguments et qu'il entretient donc une certaine proximité avec l'hyperlien explicatif, le potentiel d'action d'un hyperlien « déplaçant le focus » peut mettre au défi les attentes d'un lecteur à la recherche de simples confirmations des informations avancées dans le texte géniteur.

L'article (10) de lexpress.fr est consacré aux révisions de la loi sur la bioéthique. L'article annonce un tour d'horizon des principales questions examinées par le Parlement. La question du don d'ovules est traitée sous l'angle de la pénurie : " alors que 2000 femmes stériles auraient, chaque année, besoin d'un don d'ovocytes (cellule sexuelle qui va évoluer en ovule après maturation), en pratique seules 500 d'entre elles peuvent effectivement y accéder ». L'hyperlien ne donne pas accès à une définition médicale de ce procédé, mais à un texte dans lequel le député Jean Leonetti plaide pour la congélation d'ovocytes. Les répertoires du texte géniteur et du texte relié entretiennent un rapport de causalité certain (le texte géniteur énonce un besoin, le texte relié propose une solution), et la 
thématique abordée est la même ; le texte géniteur déplace néanmoins le focus vers un aspect particulier de la problématique sans que ce changement soit annoncé dans le texte géniteur.

Une interview (23) publiée dans lexpress.fr est consacrée au débat sur la laïcité. Jean-Christophe Lagarde, député-maire de Drancy, plaide pour l'actualisation de ce débat en évoquant en outre les prières publiques dans une rue parisienne. "La situation de la rue Myrha existe depuis 30 ans", affirme le député. Le texte relié déplace le focus en insistant moins sur les pratiques religieuses elles-mêmes que sur les commentaires de ces pratiques par Marine Le Pen et d'autres acteurs politiques. La " situation de la rue Myrha » se trouve potentiellement mise à discussion via un croisement de points de vue politiques. Il faut également souligner le fait que l'hyperlien se trouve ici inséré dans une interview, alors que rien n'annonce la volonté de l'interviewé de convoquer les propos de Marine Le Pen. L'hyperlien propose donc un déplacement de focus tout en constituant la trace d'une interprétation des paroles de l'interviewé par le journaliste.

\section{Hyperlien « comparatif »}

L'hyperlien " comparatif », autre déclinaison de l'hyperlien à potentiel d'action dialogique, propose une analogie entre deux ou plusieurs événements, personnes ou faits. Il préfigure un lecteur prêt à effectuer un transfert de caractéristiques qui nécessite un acte de lecture à la fois créatif et critique : toute comparaison pose la question de sa validité, et de ses frontières avec l'amalgame. Des annonces et commentaires dans le texte géniteur peuvent éventuellement orienter le lecteur dans le repérage des éléments convergents et divergents entre texte géniteur et texte relié.

Le texte géniteur d'un article de lexpress.fr portant sur l'affaire Pornic (8) affirme que Nicolas Sarkozy est coutumier de l'initiative de politiser des faits divers : " Et voilà le fait divers qui devient un fait politique ". L'hyperlien donne accès à une multitude d'exemples depuis 2005 où une telle transformation a eu lieu, proposant une analogie entre les cas précédents et le cas actuel. C'est au lecteur d'effectuer le travail de transfert entre le passé et le présent, c'est à lui d'en tirer des conclusions.

Une autre occurrence de l'hyperlien comparatif se trouve dans l'article (23). Dans cette interview publiée par lexpress.fr, le député-maire de Drancy affirme en outre que le débat sur la laïcité devrait être encadré d'une façon rigoureuse. Il avance que ce débat pourrait ressembler à celui 
"sur la burqa ». L'emplacement de l'hyperlien suscite potentiellement l'attente d'en savoir plus sur ce débat, mais pose aussi la question des éléments de convergence possibles avec la situation actuelle. Le texte relié déroule la chronologie du débat sur la burqa, et expose des arguments avancés à l'époque. Il incombe au lecteur de repérer les arguments qui s'appliquent à la situation actuelle. Encore une fois, cet hyperlien se trouve inséré dans les paroles de l'interviewé. L'analogie entre la situation actuelle et le débat sur la burqa est établie par le député-maire ; le journaliste approfondit cette analogie en y reliant, par hyperlien, la chronologie du débat. Se croisent donc, dans cet énoncé, plusieurs énonciateurs dont les rôles peuvent être distingués par le lecteur attentif.

\section{Hyperlien « croisant des points de vue »}

Certains hyperliens font potentiellement dialoguer des points de vue divergents. Comme je l'ai évoqué plus haut, il arrive assez fréquemment qu'aucune indication dans le texte géniteur n'annonce cette présentation de contre-arguments dans le texte relié.

Dans l'article (1) publié par lexpress.fr, le texte géniteur énonce que « la question que se posent désormais tous les économistes est de savoir quand la Chine détrônera les États-Unis et deviendra le maître du monde » (voir capture d'écran figure 9). À la seule lecture du texte géniteur, la croissance économique en Chine parait évidente, et l'accès à la première place dans l'économie mondiale ne semble être qu'une question de temps. Le texte relié, en revanche, pointe la pauvreté de certaines couches de la population chinoise. Il nuance l'argument de la forte croissance économique en Chine dès le titre en demandant : " La Chine est-elle vraiment la deuxième puissance économique mondiale ? ». Se met potentiellement en place une argumentation nuancée où différents points de vue sont mis en perspective, reflétant la complexité de la question.

L'article (22) est consacré aux actions de l'agence Frontex, dispositif mis en place par les États membres de l'UE face aux crises migratoires. Dotée d'un budget de 88 millions d'euros en 2011, l'agence étudie les mouvements migratoires, coordonne des opérations de gestion de flux et gère les vols de retour des migrants refoulés. En 2010, elle a, « selon les “eurodéputés Europe Écologie”, mené 43 opérations de contrôle ou de vols retours » : chiffre que l'auteur de l'article considère comme faible. Via l'hyperlien posé sur « eurodéputés Europe Écologie », l'Auteur donne accès au rapport complet. Ce rapport va pourtant beaucoup plus loin que 
la seule critique des chiffres : il accuse explicitement Frontex de violations des droits de l'homme. Dans le texte géniteur, le journaliste de lexpress. fr reprend uniquement la critique des chiffres. Pour la seule fois dans le texte géniteur, il cite explicitement sa source, mais ne prend donc que partiellement position vis-à-vis d'elle. Compte tenu de la reprise des chiffres, le lecteur peut supposer que l'auteur approuve la critique faite dans le rapport par les eurodéputés. Est-il également d'accord avec les autres arguments avancés par Europe Écologie, concernant notamment la violation des droits de l'homme? Aucune indication dans le texte géniteur ne permet d'en décider - sauf l'hyperlien lui-même, menant vers le rapport complet. Le lecteur s'attendant à une simple confirmation des chiffres peut être ébranlé par la violence des accusations, qui jettent potentiellement une lumière différente sur la totalité du texte géniteur (voir plus loin l'analyse exhaustive de cet article).

Figure 9. Exemple d'un hyperlien " dialogique " dans l'article (1) du corpus, qui rentre dans la catégorie de l'hypertexte " croisant les points de vue "

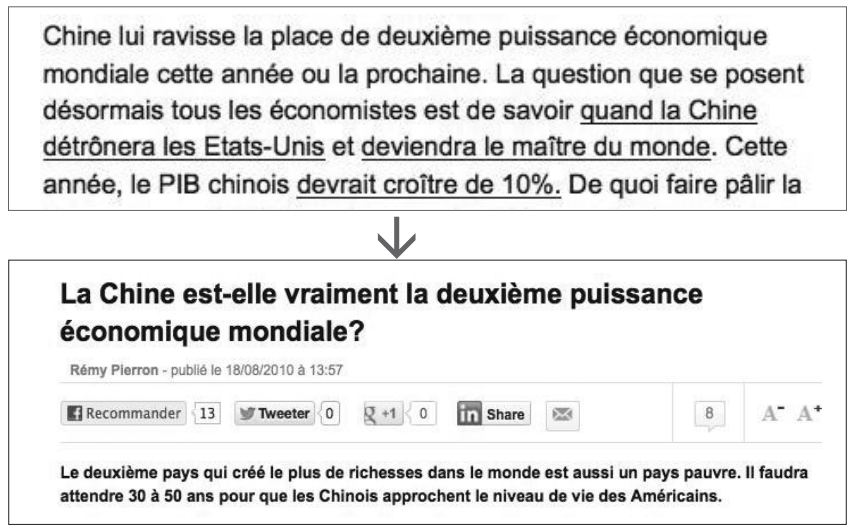

L'activation de l'hyperlien sur " quand la Chine détrônera les États-Unis » provoque l'affichage d'un article qui met en question ce pronostic.

Un exemple particulièrement complexe d'un hyperlien croisant des points de vue se trouve dans l'exemple (13). Dans cet article de marianne2.fr consacré à l'affaire Pornic (une jeune fille a été tuée par un multirécidiviste), l'auteur examine les failles de communication entre policiers et gendarmes. 
Le directeur interrégional des services pénitentiaires, en charge de suivre le multirécidiviste Tony Meilhon, a été limogé, alors que les magistrats et policiers en charge de l'affaire ne recevront finalement pas de sanction. Trois hyperliens dans la même phrase complexifient potentiellement la lecture du texte géniteur en nuançant fortement le propos avancé : "Le service pénitentiaire d'insertion et de probation de Nantes en a pris pour son grade dans les enquêtes internes du ministère ". Le premier texte relié à l'hyperlien " service pénitentiaire d'insertion et de probation de Nantes " expose les difficultés récurrentes du service (voir capture d'écran figure 10). Documents à l'appui, il cherche à prouver que le service avait alerté les autorités depuis longtemps, mais sans succès. Le deuxième hyperlien posé sur l'énoncé " en a pris pour son grade » renvoie à un article du figaro.fr explicitant et justifiant les reproches faits à ce service. Alors que le premier hyperlien dans la phrase donne donc la parole au service qui se défend des accusations, le deuxième hyperlien donne la parole aux accusateurs. Le troisième hyperlien posé sur les " enquêtes internes du ministère " mène vers un article de lexpress.fr qui fournit des compléments d'information sur le rapport de l'Inspection générale des services (IGS) tout en les juxtaposant aux arguments des accusés

Figure 10. Exemple dans l'article (13) d'un hypertexte « croisant les points de vue »

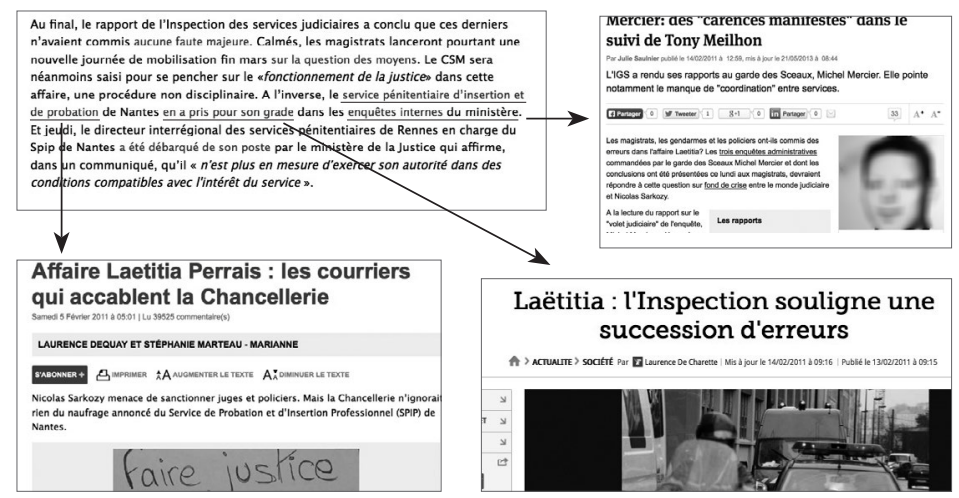

Le texte relié à l'hyperlien " service pénitentiaire d'insertion et de probation de Nantes » expose les difficultés récurrentes du service. Le deuxième hyperlien posé sur l'énoncé « en a pris pour son grade » renvoie vers un article explicitant et justifiant les reproches faits à ce service. Le troisième hyperlien posé sur les "enquêtes internes du ministère " mène vers un article fournissant des compléments d'information sur le rapport de l'IGS tout en les juxtaposant aux arguments des accusés. 
Les trois hyperliens permettent donc au lecteur de faire un tour complet de la problématique, exposée selon différents points de vue. Le fait que seul le premier hyperlien est interne peut être interprété comme une trace subtile du positionnement de l'Auteur. Même s'il approuve donc plutôt les arguments plaidant en faveur du service pénitentiaire, il invite pourtant le lecteur à accéder aux accusations formulées dans le figaro.fr et à se forger son propre avis.

\section{Hyperlien « réinterprétant »}

L'hyperlien « réinterprétant » constitue un cas limite des figures de la lecture dialogique. Il permet potentiellement au lecteur de voir comment un événement politique, un chiffre ou un fait divers ont été réinterprétés par l'Auteur à partir d'une information-source, sans que la divergence des points de vue ne soit annoncée dans le texte géniteur. Le potentiel d'action d'un tel hyperlien pose parfois problème. Certes, l'hyperlien permet au lecteur scrupuleux de comparer source et réinterprétation, et de démasquer la stratégie argumentative du journaliste. Tout auteur de textes journalistiques sait cependant que les hyperliens ne sont pas systématiquement activés, et que beaucoup de lecteurs approchent l'hyperlien avec une présomption d'information. La réinterprétation risque donc de passer inaperçue, même si l'Auteur se prémunit de tout reproche de manipulation en donnant au lecteur la possibilité de consulter la source.

Dans l'article (1) de lexpress.fr consacré à la visite du président chinois $\mathrm{Hu}$ Jintao en France, beaucoup de textes reliés nuancent les propos du texte géniteur. Le lecteur a donc potentiellement une vision différente sur les questions abordées par le texte géniteur s'il active les hyperliens, ou s'il décide de ne pas cliquer. Concernant les relations économiques entre la France et la Chine, l'auteur cite un chiffre officiel en avançant : «Seules 1400 entreprises françaises sont implantées en Chine, selon le Quai d'Orsay ». Dans le texte relié, le Quai d'Orsay présente pourtant ce chiffre comme satisfaisant. Si le lecteur procède à une lecture dialogique mettant en perspective les points de vue exposés, il constatera non seulement que ce chiffre économique peut être interprété selon deux points de vue divergents ; il comprendra aussi comment un discours officiel se trouve parfois réinterprété par un média dans le cadre d'une stratégie d'argumentation critique.

Cette méta-lecture portant sur les interprétations possibles du réel par le texte numérique est non seulement un enjeu important pour la 
recherche sur les stratégies de communication du texte numérique, mais peut utilement être intégrée dans une formation à la culture des médias.

Figures de la lecture « immersive »

L'hyperlien ne fait pas que relier deux textes, il est aussi un outil manipulable. Comme je l'ai précisé au premier chapitre, certains enchaînements de gestes peuvent être considérés comme le signifiant d'un signe iconique appelé unité sémiotique de la manipulation. L’unité " activer ", impliquée dans l'hyperlien, est par exemple composée d'un geste d'appui et de relâchement bref et non répétitif sur une zone manipulable - indépendamment du fait que cet enchaînement de gestes s'effectue sur les touches d'une souris, sur un touchpad ou sur un écran tactile. Un tel énoncé de gestes fait appel à des processus d'intégration et de stabilisation d'expériences antérieures. L'unité « activer » pourrait ainsi renvoyer à l'appui sur des boutons ou des interrupteurs, et convoquer de façon sensible des idées de réactivité, de maîtrise et de déclenchement instantané.

L'actualisation du potentiel d'action de cette forme-modèle du texte s'effectue en fonction du texte auquel elle est couplée, en fonction du contexte et en fonction de l'horizon d'attente du lecteur. J'ai parlé d'irradiation iconique lorsque le potentiel d'action de l'unité sémiotique de la manipulation se couple au potentiel d'action du texte pour souligner, préciser, renforcer, métaphoriser ou déstabiliser celui-ci, formant des procédés rhétoriques qui anticipent sur des pratiques de lecture. Grâce au caractère manipulable de l'hyperlien, le texte hyperlié peut par exemple se transformer en simulacre de référent. La manipulabilité du texte numérique crée dans ce cas une relation forte entre le technologique et le sensoriel. En résulte une présentification qui incite le lecteur à mettre de côté, au moins transitoirement, son incrédulité vis-à-vis des pouvoirs de représentation du texte. Certains couplages entre textes et unités sémiotiques de la manipulation préfigurent ainsi des pratiques de lecture immersives.

L'expérimentation avec les figures de la lecture immersive n'est pas sans risque, comme je l'ai déjà fait remarquer plus haut. D'une part, l'immersion a souvent été approchée avec suspicion : elle serait l'apanage du lecteur naif, à la recherche de sensations fortes qui le divertissent des enjeux politiques, économiques et sociétaux de son époque. D'autre part, les procédés de l'immersion et de la présentification se trouvent généralement associés à la fiction. 
Le potentiel d'action de l'hyperlien immersif dans le journalisme convoque jusqu'à un certain point les expériences proposées au lecteur par les immersive news. Dans cette forme de journalisme encore peu expérimentée en France, le lecteur est invité, au lieu de lire par exemple un rapport sur une intervention militaire, à endosser lui-même le rôle d'un soldat traversant une ville assiégée ; il peut, par avatar interposé, éprouver la peur de tomber dans une embuscade ; en activant des armes par des gestes de manipulation sur le dispositif numérique, il est amené à participer mentalement $e t$ physiquement à l'expérience de combat [voir exemples cités par Nolan, 2003]. De telles expériences ne sont certes pas présentes dans les textes étudiés ici. J'ai néanmoins pu observer que certains magazines comme lexpress.fr permettent parfois au lecteur de géolocaliser des lieux de crime sur Google Maps. En activant le nom d'un village, le lecteur se trouve littéralement projeté sur le lieu du crime ; en activant la street view, il explore les alentours par ses gestes.

D'un côté, l'immersion corporelle dans les événements relatés abolit potentiellement la distance entre le lecteur et les événements représentés, et crée peut-être un surplus d'empathie. D'un autre côté, cette abolition des distances pourrait provoquer une banalisation, voire une fictionnalisation de la réalité. Dans le corpus journalistique étudié, les expérimentations de l'hyperlien immersif, qui pourraient constituer un point de départ vers le « Nouveau Journalisme » [Wolfe, 1973] impliquant le corps du lecteur, sont encore marginales.

\section{« Kiné-gramme »}

J'appelle « kiné-gramme » le procédé qui donne potentiellement l'impression au lecteur de manipuler aussi l'objet ou le concept évoqués, et non pas seulement le mot. L'irradiation iconique apportée par l'unité sémiotique de la manipulation transforme le texte au moins partiellement en simulacre de référent.

"Pas question d'aborder les thèmes sensibles des droits de l'homme ou de la sous-évaluation du yuan ", écrit le journaliste de lexpress.fr dans l'article (1), résumant certains interdits lors de l'entrevue entre Nicolas Sarkozy et le président chinois Hu Jintao (voir captures d'écran figure 11). L'auteur aborde pourtant la question des droits de l'homme passée sous silence entre les deux chefs d'État, en mobilisant une figure de rhétorique 
textuelle appelée "pérétrition $»^{5}$. De surcroît, l'hyperlien sur l'énoncé « aborder les thèmes sensibles des droits de l'homme » rend celui-ci manipulable par le lecteur.

Figure 11. Exemple d'un hyperlien qui entre dans la catégorie du « kiné-gramme »

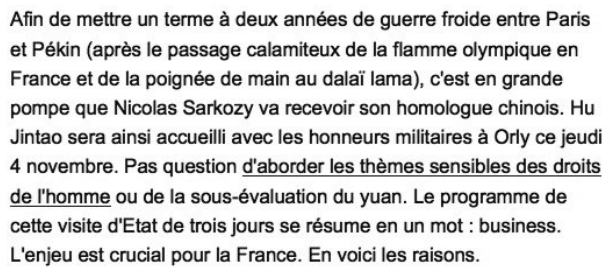

En activant l'hyperlien sur " d'aborder les thèmes sensibles des droits de l'homme », le lecteur n'a-t-il pas l'impression d'aborder lui-même les thèmes sensibles par son geste?

Les thèmes des droits de l'homme sont qualifiés de « sensibles ». L'unité « activer » renvoie potentiellement à des idées de déclenchement et de réactivité instantanés. Le texte relié traite, entre autres, de la question des droits de l'homme en Chine passée sous silence entre les deux chefs d'État. Grâce à sa manipulabilité, l'énoncé hypertextualisé suggère donc au lecteur qu'il peut aborder lui-même, par son appui sur des " points sensibles ", les thèmes tabous entre les deux présidents, et provoquer des réactions. L'irradiation iconique induite par l'unité " activer » donne une dimension corporelle à l'acte d'aborder, que je propose d'interpréter comme une tentative d'impliquer le lecteur plus profondément dans le débat. Le kiné-gramme pourrait dans cet exemple également créer une certaine complicité entre auteur et lecteur : ce qui est passé sous silence dans les discours officiels est littéralement pointé par un geste de résistance - un geste qui creuse les failles et les non-dits.

5. Dans la pérétrition, l'énonciateur déclare qu'il passera sous silence une chose sur laquelle il attire pourtant l'attention, en l'énonçant. 
Figures de la lecture « déviative »

Dans certains exemples du corpus, des lieux d'indétermination entre texte géniteur et texte relié, ou texte et unités sémiotiques de la manipulation, se creusent : se trouvent reliés des textes, ou des textes et formesmodèles qui, de première apparence, n’ont pas grand-chose à voir.

$\mathrm{Au}$ niveau du couplage entre texte et formes-modèles, la lecture immersive se trouve dans ce cas à la fois encouragée et minée. Au niveau de la relation entre texte géniteur et texte relié, ce manque de contiguïté peut se manifester à travers des sauts temporels importants et non expliqués, des brisures dans l'enchaînement causal, et des divergences entre les répertoires et stratégies textuels déstabilisant le lecteur.

Les figures de la lecture que je propose d'appeler « déviative » créent donc potentiellement des surprises déconcertantes et des découvertes insolites, qui peuvent éventuellement subvertir très fortement l'argumentation du texte géniteur. Une telle relation a peu de chances de répondre aux attentes du lecteur, qu'elles soient informationnelles ou dialogiques - d'autant plus si le texte géniteur ne prépare pas le terrain à la découverte insolite, et si le texte relié ne confirme pas ce que le texte géniteur a annoncé. Certaines de ces figures font donc appel à un lecteur prêt à se laisser surprendre, et à s'engager dans un processus d'interprétation remédiant au moins partiellement au manque de contiguïté. D’autres figures semblent anticiper sur un lecteur naïf, dont la crédulité peut être abusée par une argumentation manipulatrice.

Il est en effet parfois difficile de décider si les procédés rhétoriques de cette figure relèvent d'une volonté d'abuser de la présomption d'information du lecteur, s'ils renvoient à une tentative volontaire de rompre les schémas de pensée familiers et d'inciter au décryptage critique de structures de domination pesant sur la fabrique de l'information, ou s'ils constituent simplement une erreur d'aiguillage.

Reste aussi à savoir si, dans un contexte de réception journalistique, le lecteur veut se prêter au jeu de la figure déviative. Pour un lecteur qui attend du journalisme la mise à disposition d'informations neutres et objectives, la déviation de ses attentes dépasse peut-être les limites de l'acceptable. Même un lecteur qui attend de l'hyperlien un croisement d'opinions divergentes se sentira peut-être déconcerté par certains de ces hyperliens. 


\section{Hyperlien « déformatoire »}

Je propose de parler d'un potentiel d'action « déformatoire » lorsque l'activation de l'hyperlien mène vers un texte relié qui présente des éléments qui contredisent les affirmations dans le texte géniteur, sans que ces contradictions soient explicitement annoncées ou commentées. L'hyperlien renvoie par exemple à une source d'information, mais celleci est réinterprétée jusqu'à déformation complète. La frontière entre la manipulation préméditée, et l'éventuelle volonté de contester des discours d'autorité n'est pas toujours facile à tracer. L'interprétation d'un tel hyperlien dépend donc fortement de l'horizon d'attente du lecteur.

Dans l'article (12) publié par lepost.fr que j'ai déjà convoqué au chapitre I, le journaliste revient sur le choix des candidats pour l'émission télé Parole de Français avec Nicolas Sarkozy (voir figure 12). Le journaliste rappelle certains détails concernant les candidats de l'année précédente. Les propos d'une jeune auto-entrepreneuse ont été complaisants : " on apprenait par la suite qu'elle était fan de François Fillon ", commente le journaliste. Le texte relié indique pourtant dès le titre que la femme conteste être fan de François Fillon et dénonce une stratégie de manipulation médiatique. Le journaliste du post.fr veut-il perpétuer cette manipulation tout en se prémunissant, par l'insertion d'un hyperlien, d'un éventuel reproche de diffamation ? Ou veut-il dénoncer le démenti de la femme comme un mensonge ?

Figure 12. Exemple d'un hyperlien « déformatoire » dans l'article (12) du corpus

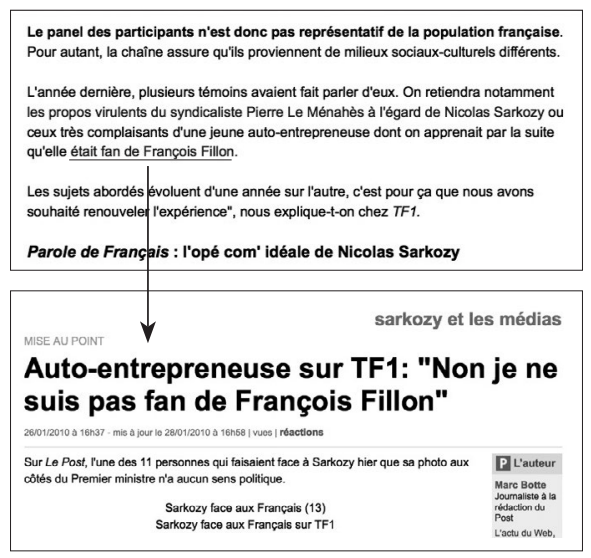

L'activation de l'hyperlien sur " était fan de François Fillon " provoque l'affichage d'un article où la jeune femme conteste vivement cette affirmation. 
Lors de la visite de Mouammar Kadhafi en France, l'Élysée avait promis la mise en place de contrats de plusieurs milliards d'euros pour justifier l'événement. Un article (15) de lexpress.fr se penche sur les résultats de cette visite qui, selon l'auteur, a été " dealée lors des négociations pour la libération des infirmières bulgares en juillet 2007 ». Si le lecteur approche cet hyperlien avec une présomption d'information, il s'attend sans doute à une confirmation de ce "deal » par texte relié. Si ses attentes vont dans le sens d'une juxtaposition dialogique de points de vue, le lecteur espère peut-être accéder au débat autour de ces négociations. Dans le texte relié datant de 2007, Nicolas Sarkozy nie pourtant l'existence même d'une contrepartie autre que financière pour la libération des infirmières bulgares. L'argument que cette visite d'État a été « dealée » par Nicolas Sarkozy déforme donc fondamentalement les affirmations du chef d'État. En reliant l'article à cette source, l'Auteur veut peut-être accuser Nicolas Sarkozy d'une déformation des faits et inciter au décryptage de ce mensonge. Le potentiel d'action de ce procédé reste néanmoins ambigu : beaucoup de lecteurs approchent spontanément l'hyperlien avec des attentes informationnelles qui, si elles ne sont pas vérifiées par l'activation de l'hyperlien, risquent de faire passer le lecteur à côté du démenti par le chef d'État.

\section{Hyperlien « ironisant »}

Dans l'hyperlien à potentiel d'action " ironisant ", la rétroprojection d'éléments du texte relié sur le texte géniteur substitue potentiellement à certains termes leur " propre négation » [Klinkenberg, 1996, 361]. La question de savoir si l'oscillation entre affirmation et négation, si typique de l'ironie, se trouve apaisée ou renforcée par l'hyperlien dépend des effets d'annonce, de l'emplacement de l'hyperlien, des répertoires et stratégies textuels et des figurations potentiellement mobilisées par l'hyperlien et par le média.

L'article (24) de lexpress.fr revient sur les visites de Nicolas Sarkozy consacrées à l'histoire de France, et montre qu'un conseiller du chef d'État était à l'origine de cette idée : " Patrick Buisson ", qualifié par le journaliste d'« éminent conseiller ». À la seule lecture du texte géniteur, une interprétation littérale du mot " éminent » semble possible. Le texte relié, en revanche, renverse potentiellement l'idée que le lecteur se fait d'un conseiller " éminent »: dans le contexte du paiement des sondages à l'UMP, Patrick Buisson serait mis en cause pour une convention entre 
l'Élysée et sa propre entreprise. Par rétroprojection de ces éléments sur le texte géniteur, le qualificatif « éminent » se trouve donc potentiellement mis en doute. L'actualisation plus ou moins complète de ce renversement ironique dans une situation de lecture précise dépend du point de vue du lecteur, et des imaginaires dont il investit le magazine lexpress.fr.

L'article (3) du post.fr traite du duel Dominique Strauss-Kahn - Nicolas Sarkozy, auquel les médias s'attendaient début 2011. Il débute d'une façon qui suggère déjà que le potentiel d'action de certains adjectifs pourrait être ironique : "Surprise, lundi, lors du discours du G20/G8 avec vœux au corps diplomatique incorporés : Nicolas Sarkozy, circonspect, posé, pédagogique (voire pontifiant), mesuré et précautionneux, s'abstient de toute annonce spectaculaire... ». Le texte relié présente un photomontage où la tête du chef d'État se trouve collée sur le corps de Louis de Funès dans le film L'aile ou la cuisse.

Le nom propre de l'homme politique et la photographie de l'acteur font appel à des répertoires supposés partagés en France. Nicolas Sarkozy est chef d'État au moment de la publication de l'article. Charles Duchemin est directeur d'un guide gastronomique. L'un est certes un personnage de l'actualité politique, tandis que l'autre est un personnage de fiction. Les deux partagent néanmoins des qualités anticipées comme étant familières au lecteur. Ces qualités contredisent les adjectifs " posé » et "pédagogique » dans le texte géniteur tout en rapprochant les deux personnages. Le lieu d'indétermination entre texte géniteur et texte relié, fondé en outre sur la divergence entre réalité et fiction, pourrait donc se résoudre par l'hypothèse d'une inversion du sens. Comme Charles Duchemin dans le film L'aile ou la cuisse, Nicolas Sarkozy ne serait en réalité nullement posé et pédagogique.

Le texte relié fait plus que " confirmer » ce renversement ironique. Il établit aussi une analogie potentielle entre un chef d'État et un acteur, qui pourrait suggérer que l'attitude de Nicolas Sarkozy relève de la pose d'acteur.

L'hyperlien ironisant n'est pas le seul à rappeler des procédés rhétoriques du texte papier. Pour compléter le relevé du champ des possibles, il me paraît utile de convoquer l'exemple d'un hyperlien " métaphorisant » repéré dans un blog littéraire.

\section{Hors corpus : hyperlien « métaphorisant »}

Plus haut, j’ai cité la comparaison établie par Lev Manovich [2001, 228] entre le potentiel d'action de certains hyperliens et la métonymie. 
Dans ces hyperliens, le mot hyperlié vaut pour le texte relié ; il le représente d'une façon prévisible. L'hyperlien " métaphorisant », en revanche, " éclaire » les éléments du texte géniteur et en même temps les " éclaire à l'infini » (voir la définition de la métaphore par Roland Barthes [1971, 95]). La relation entre texte géniteur et texte relié ne se laisse donc que partiellement épuiser par un processus d'interprétation. L'hyperlien métaphorique préfigure un lecteur prêt à déployer son imagination, à explorer des hypothèses, à se laisser surprendre par des associations inattendues et à s'engager dans une lecture interprétative des textes proposés.

Sur son blog tierslivre.fr, François Bon discute la question de savoir « comment Internet multiplie la littérature » :

Ce qui est fascinant, côté Internet, c'est le lien qu'on peut constituer de pair à pair (peer to peer), l'idée de réticule commence par une auto prise en charge : elle s'annonce déjà trop tardive. Internet ne peut fonctionner selon des utopies de maison commune, aussi parce qu'il s'ancre désormais sur des lieux de création singulière, dont le principe serait plutôt de constellation, et que ce qui s'y affirmera peu à peu comme contenu de littérature le fera depuis ces singularités, par quoi en chacune le langage va au monde ${ }^{6}$.

Le mot « réticule » n'est pas courant. En activant l'hyperlien, le lecteur pourrait s'attendre à une définition du terme, qui désigne à la fois une constellation de l'hémisphère Sud, et un dispositif optique permettant d'effectuer des visées précises en interposant dans le champ visuel net une croisée de fils traçant un repère. Dans le texte géniteur, le mot est déjà utilisé comme une métaphore qui fait jouer les deux significations du terme : le système du "peer to peer" est à la fois rapproché de l'idée de constellation (reprise plus loin dans le texte), et de l'idée d'une visée précise. L'activation du mot " réticule " projette le lecteur non pas vers une définition du terme, mais vers la première page du blog LittéRéticulaire, journal personnel de Patrick Rebollar. L'auteur de ce blog explique le choix du titre en avançant une définition du terme " littéréticulaire »: " propriété d'un texte où s'associent, aux valeurs traditionnelles et aux figures classiques du texte littéraire, les significations et effets de sens provoqués par les liens hypertextuels au sein d'un réseau (l'Internet par exemple), qu'ils aient été voulus ou non par l'auteur ».

6. François Bon, Le tiers livre, [En ligne] : < http://www.tierslivre.net/spip/spip.php?article676 >. 
D’une part, le renvoi vers un autre blog pourrait être interprété comme une illustration du "peer to peer" selon le principe de la visée précise, donc comme la focalisation d'une singularité. Cette interprétation laisse pourtant de côté l'idée de " constellation », reprise quelques mots après le terme « réticule » par un deuxième hyperlien. Lorsque le lecteur active cet hyperlien sur « constellation », l'image interactive d'un ciel étoilé s'affiche ; chaque activation d'un point lumineux mène vers un site personnel. La deuxième signification du terme " réticule », qui semble écartée dans la relation établie entre le mot " réticule » et le blog LittéRéticulaire, se trouve donc réactivée par le contexte de l'hyperlien.

L'hyperlien " réticule » est ainsi potentiellement caractérisé par une oscillation permanente entre les significations " repère » et « constellation », qui n'est que momentanément interrompue par le renvoi à un blog précis. Ce renvoi fait en outre intervenir la troisième signification du terme " réticule », désignant chez Patrick Rebollar la fonction de l'hyperlien en tant que procédé rhétorique. Si je projette cette signification possible sur l'hyperlien « réticule », celui-ci se trouve investi de l'idée de constituer une figure-phare de la sphère littéraire sur Internet. L'hyperlien sur « réticule » fait donc expérimenter au lecteur, par son fonctionnement même, le fondement de la sphère littéraire numérique en tant que visée précise et en tant que constellation. Cette interprétation est relativement totalisante : en fonction de mes propres connaissances du principe "peer to peer", je l'estime recevable.

En même temps, il suffit que je recommence la lecture de l'extrait du tierslivre pour que l'oscillation entre « visée précise » et « constellation » recommence, et sollicite de nouveau mon imagination. Le fait que le texte relié, le journal LittéRéticulaire, se transforme presque quotidiennement et m'apparaît donc autre à chaque actualisation, joue un rôle important dans le caractère inépuisable de cet hyperlien.

Par ailleurs, si je prends en compte le fait que le « réticule » est aussi un outil pour viser, je me demande jusqu'à quel point la manipulabilité de l'hyperlien agit sur le mot « réticule » et le transforme peut-être, au moins partiellement, en outil : est-ce que je ne vise pas aussi très littéralement le journal LittéRéticulaire en activant l'hyperlien?

\section{« Kiné-trope »}

Je viens maintenant aux couplages entre textes et unités sémiotiques de la manipulation qui relèvent potentiellement d'une figure de la lecture déviative. 
Dans l'article (3) du post.fr consacré au duel entre Nicolas Sarkozy et Dominique Strauss-Kahn, auquel les médias se préparaient début 2011 pour la présidentielle de 2012, le journaliste fait allusion à une couverture de Newsweek présentant Dominique Strauss-Kahn comme futur président de la République : "De sauveur, le monde en avait pourtant déjà un, Français de surcroît, et fort apprécié des Américains et de toute la planète (à en croire Newsweek, souvenez-vous) ».

L'énoncé « souvenez-vous » renvoie potentiellement à l'idée de ramener un objet ou un événement à la mémoire. L'unité « activer », couplée à l'injonction " souvenez-vous », peut être associée à des idées de déclenchement et de réactivité instantanés, qui rappellent potentiellement au lecteur des gestes comme l'appui sur un interrupteur ou un bouton. Or, le ressouvenir ne constitue pas, en principe, un processus activable par simple appui physique. Même si l'« outil » du ressouvenir électronique fonctionne en affichant effectivement la couverture de Newsweek, la mémoire du lecteur n'a sans doute pas fonctionné ainsi.

Un lieu d'indétermination possible s'ouvre donc entre le potentiel d'action de l'icône manipulable, et le potentiel d'action de l'injonction « souvenez-vous » adressée au lecteur. En activant cette injonction, le lecteur peut néanmoins avoir l'impression de se souvenir aussi lui-même.

Pour interpréter le rapprochement établi par ce couplage entre ressouvenir machinique et mémoire humaine, il me paraît cohérent de convoquer une figuration de l'Internet assez répandue dans les années 1990 et 2000. À cette époque, plusieurs auteurs ont avancé que l'espace d'écriture électronique, et en particulier le potentiel « associatif » de l'hyperlien, reflète la façon dont fonctionne l'esprit humain, rendant explorables des connexions entre idées (voir chapitre II). L'hyperlien « souvenezvous » dans l'exemple cité pourrait renvoyer à cette figuration de l'Internet comme mémoire-prothèse. Le lecteur est censé se souvenir, mais au lieu d'activer sa mémoire personnelle, il consulte la mémoire de l'Internet en appuyant sur des hyperliens.

Je propose de donner encore du fondement à cette hypothèse d'interprétation en rappelant que le fonctionnement des neurones se trouve souvent comparé à celui de flux électriques ${ }^{7}$, et que le système nerveux est parfois stimulé par des électrodes lors d'examens médicaux. Le couplage entre le texte et l'icône dans l'énoncé « souvenez-vous » peut donc être

7. Voir par exemple : « Les neurones assurent la transmission d'un signal bioélectrique appelé influx nerveux », [En ligne] : < http://fr.wikipedia.org/wiki/Neurone >. 
interprété comme matérialisation de l'idée qu'un souvenir s'active aussi facilement que la lumière s'allume, par appui sur un bouton. L'invariabilité technologique de la relation hypertextuelle renforcerait l'idée que l'activation de cette relation assure la restitution inaltérée de la mémoire. Comme il est jouissif de faire fonctionner l'outil, et de s'immerger pour un court instant dans le rêve d'une fusion des mémoires entre l'homme et la machine !

Pourtant, le lecteur sait bien que le souvenir humain ne fonctionne pas ainsi. La persistance de ce décalage dans le couplage entre les potentiels d'action du texte et de sa forme-modèle continue à troubler l'immersion. Comme ce décalage rappelle les processus d'émergence de la signification dans certaines figures tropologiques du texte papier, je propose d'appeler ce procédé « kiné-trope ».

Le tableau ci-dessous liste encore une fois les procédés rhétoriques de l'hyperlien identifiés dans le corpus journalistique, et les figures de la lecture sur lesquelles ces procédés anticipent.

\section{Figures de la lecture de l'hyperlien et ses procédés}

\section{Tableau 2. Figures de la lecture informationnelle}

\begin{tabular}{|c|c|}
\hline $\begin{array}{l}\text { Hyperlien définissant } \\
\text { préfigure un lecteur désireux d'en } \\
\text { savoir plus sur des mots et notions. }\end{array}$ & $\begin{array}{l}\text { Hyperlien posé sur des mots } \\
\text { rares, des notions complexes ; le } \\
\text { texte relié propose la définition } \\
\text { d'un terme technique, d'un } \\
\text { dispositif, d'une institution... }\end{array}$ \\
\hline $\begin{array}{l}\text { Hyperlien renvoyant à la source } \\
\text { préfigure un lecteur souhaitant vérifier } \\
\text { ce qui se trouve énoncé dans la source. }\end{array}$ & $\begin{array}{l}\text { Mise en relation du texte géniteur } \\
\text { avec la source qui a permis à l'Auteur } \\
\text { de circonscrire les contours d'un } \\
\text { événement, d'une personnalité... }\end{array}$ \\
\hline $\begin{array}{l}\text { Hyperlien illustratif } \\
\text { préfigure un lecteur souhaitant accéder } \\
\text { à une confirmation de l'information } \\
\text { donnée dans le texte géniteur. }\end{array}$ & $\begin{array}{l}\text { Mise en relation de certains } \\
\text { éléments du texte géniteur avec } \\
\text { des illustrations visuelles. }\end{array}$ \\
\hline $\begin{array}{l}\text { Hyperlien fournissant des preuves } \\
\text { préfigure un lecteur voulant disposer } \\
\text { de preuves pour l'information } \\
\text { donnée dans le texte géniteur. }\end{array}$ & $\begin{array}{l}\text { Le texte relié confirme les affirmations } \\
\text { avancées dans le texte géniteur } \\
\text { par des preuves factuelles. }\end{array}$ \\
\hline
\end{tabular}




\begin{tabular}{|l|l}
$\begin{array}{l}\text { Hyperlien d'autorité } \\
\text { préfigure un lecteur se laissant } \\
\text { rassurer par une « parole d'expert ». }\end{array}$ & $\begin{array}{l}\text { En renvoyant à des personnalités } \\
\text { ou des institutions jouissant d'une } \\
\text { position d'autorité, l'Auteur espère } \\
\text { doter le texte géniteur d'une } \\
\text { crédibilité supplémentaire. }\end{array}$ \\
$\begin{array}{l}\text { Hyperlien explicatif } \\
\text { peut répondre aux attentes } \\
\text { de lecteurs cherchant des } \\
\text { compléments d'information. }\end{array}$ & $\begin{array}{l}\text { Le texte relié décrit les circonstances } \\
\text { d'un événement, l'explique en avançant } \\
\text { des données factuelles forte contiguïté } \\
\text { causale avec le texte géniteur. }\end{array}$ \\
\end{tabular}

\section{Tableau 3. Figures de la lecture dialogique}

\begin{tabular}{|c|c|}
\hline $\begin{array}{l}\text { Hyperlien déplaçant le focus } \\
\text { préfigure un lecteur désireux } \\
\text { d'accéder à un aspect différent de } \\
\text { celui exposé dans le texte géniteur. }\end{array}$ & $\begin{array}{l}\text { Le texte relié déplace potentiellement } \\
\text { le focus du texte géniteur en } \\
\text { ciblant un aspect particulier de } \\
\text { la problématique générale, ou en } \\
\text { présentant un point de vue connexe. }\end{array}$ \\
\hline $\begin{array}{l}\text { Hyperlien comparatif } \\
\text { préfigure un lecteur prêt à effectuer } \\
\text { le transfert de caractéristiques entre } \\
\text { le texte géniteur et le texte relié. }\end{array}$ & $\begin{array}{l}\text { Texte géniteur et texte relié proposent } \\
\text { une analogie entre deux ou plusieurs } \\
\text { événements, personnes ou faits. }\end{array}$ \\
\hline $\begin{array}{l}\text { Hyperlien croisant des points de vue } \\
\text { préfigure un lecteur acceptant de mettre } \\
\text { en perspective les arguments exposés. }\end{array}$ & $\begin{array}{l}\text { Hyperlien faisant potentiellement } \\
\text { dialoguer des points de vue divergents } \\
\text { entre le texte géniteur et le texte relié. }\end{array}$ \\
\hline $\begin{array}{l}\text { Hyperlien réinterprétant } \\
\text { préfigure un lecteur suffisamment } \\
\text { attentif pour démasquer la } \\
\text { réinterprétation, ou un lecteur qui } \\
\text { se laisse manipuler en passant } \\
\text { vite sur cette réinterprétation. }\end{array}$ & $\begin{array}{l}\text { Un événement, un chiffre ou un fait } \\
\text { divers ont été réinterprétés par l'Auteur } \\
\text { à partir d'une information-source, sans } \\
\text { que la divergence de points de vue } \\
\text { soit annoncée dans le texte géniteur. }\end{array}$ \\
\hline
\end{tabular}




\section{Tableau 4. Figures de la lecture immersive}

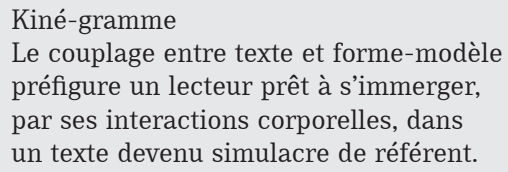

L'irradiation iconique apportée par l'unité sémiotique de la manipulation donne l'impression de pouvoir transformer le texte en simulacre de référent.

\section{Tableau 5. Figures de la lecture déviative}

\begin{tabular}{|c|c|}
\hline $\begin{array}{l}\text { Hyperlien déformatoire } \\
\text { préfigure un lecteur pressé, habitué aux } \\
\text { hyperliens informationnels ; ou alors } \\
\text { un lecteur complice, partageant avec } \\
\text { l'Auteur une volonté de subversion. }\end{array}$ & $\begin{array}{l}\text { Le texte relié présente des éléments } \\
\text { contredisant les affirmations du texte } \\
\text { géniteur, sans que ces contradictions } \\
\text { soient annoncées. La frontière } \\
\text { entre manipulation préméditée, } \\
\text { et volonté de décrypter de façon } \\
\text { critique des discours officiels n'est } \\
\text { pas toujours facile à tracer. }\end{array}$ \\
\hline $\begin{array}{l}\text { Hyperlien ironisant } \\
\text { préfigure un lecteur complice, } \\
\text { prêt à effectuer le renversement } \\
\text { ironique de certains termes. }\end{array}$ & $\begin{array}{l}\text { La rétroprojection d'éléments du texte } \\
\text { relié sur le texte géniteur y substitue } \\
\text { potentiellement leur négation. }\end{array}$ \\
\hline $\begin{array}{l}\text { Hyperlien métaphorisant } \\
\text { préfigure un lecteur prêt à déployer son } \\
\text { imagination, à se laisser surprendre } \\
\text { par des associations inattendues. }\end{array}$ & $\begin{array}{l}\text { L'hyperlien métaphorisant « éclaire » } \\
\text { des éléments du texte géniteur, et en } \\
\text { même temps les « éclaire à l'infini ». } \\
\text { La relation entre texte géniteur et texte } \\
\text { relié ne se laisse que partiellement } \\
\text { « épuiser » par l'interprétation. }\end{array}$ \\
\hline $\begin{array}{l}\text { Kiné-trope } \\
\text { le couplage entre texte et forme- } \\
\text { modèle préfigure un lecteur acceptant } \\
\text { l'oscillation entre immersion par } \\
\text { interaction corporelle et distance. }\end{array}$ & $\begin{array}{l}\text { L'irradiation iconique apportée } \\
\text { par l'unité sémiotique de la } \\
\text { manipulation agit sur le texte. Un } \\
\text { décalage persiste néanmoins entre } \\
\text { les potentiels d'action du texte et } \\
\text { de l'icône, et trouble l'immersion. }\end{array}$ \\
\hline
\end{tabular}


Les tableaux ci-après montrent comment les trois médias du corpus, lexpress.fr, marianne2.fr et lepost.fr, préfigurent leurs lectures en privilégiant certains procédés rhétoriques de l'hyperlien.

Tableau 6. Figures de la lecture informationnelle $67 \%$

\begin{tabular}{|c|c|c|}
\hline \multicolumn{3}{|c|}{ Hyperliens illustratifs 4 (2 \%) } \\
\hline lexpress.fr 2 (2 \%) & marianne2.fr $2(2,5 \%)$ & lepost.fr - \\
\hline \multicolumn{3}{|c|}{ Hyperliens renvoyant à la source 35 (16 \%) } \\
\hline lexpress.fr $16(15,5 \%)$ & marianne2.fr $13(15,5 \%)$ & lepost.fr $6(16,5 \%)$ \\
\hline \multicolumn{3}{|c|}{ Hyperliens fournissant des preuves $8(3,5 \%)$} \\
\hline lexpress.fr $5(4,5 \%)$ & marianne2.fr $2(2,5 \%)$ & lepost.fr $1(3 \%)$ \\
\hline \multicolumn{3}{|c|}{ Hyperliens d'autorité 5 (2%) } \\
\hline lexpress.fr 1 (1 \%) & marianne2.fr $3(3,5 \%)$ & lepost.fr $1(3 \%)$ \\
\hline \multicolumn{3}{|c|}{ Hyperliens définissants 13 (6 \%) } \\
\hline lexpress.fr $6(5,5 \%)$ & marianne2.fr 7 (8,5 \%) & lepost.fr - \\
\hline \multicolumn{3}{|c|}{ Hyperliens explicatifs 85 (38 \%) } \\
\hline lexpress.fr 36 (33\%) & marianne2.fr 33 (40 \%) & lepost.fr 16 (44\%) \\
\hline
\end{tabular}

Satisfaisant potentiellement la présomption d'information avec laquelle beaucoup de lecteurs approchent l'hyperlien, les figures de la lecture informationnelle priment dans les trois médias étudiés (67\%), comme le montre le premier tableau. Il faut néanmoins souligner que dans cette catégorie de figures, les hyperliens à potentiel d'action explicatif sont les plus fréquents. Notamment lepost.fr et marianne2.fr anticipent ainsi sur la figure d'un lecteur qui souhaite obtenir plus qu'une simple confirmation des informations du texte géniteur lorsqu'il clique. Les formes-modèles pro-intensives de marianne2.fr, comme la police de caractère avec empattements, et la séparation stricte entre texte à lire et commentaires peuvent favoriser cette pratique de lecture. 
Les pratiques de lecture rapides, potentiellement encouragées par les formes-modèles pro-extensives primant dans lexpress.fr et dans lepost.fr, se trouvent en revanche plutôt confirmées par les hyperliens renvoyant à la source, également fréquents dans les trois médias (16\% en moyenne).

Tableau 7. Figures de la lecture dialogique $29 \%$

\begin{tabular}{|c|c|c|}
\hline lexpress.fr 37 (19 \%) & marianne2.fr 10 (12 \%) & lepost.fr $3(8,5 \%)$ \\
\hline \multicolumn{3}{|c|}{ Hyperliens comparatifs 4 (2 \%) } \\
\hline lexpress.fr $4(3,4 \%)$ & marianne2.fr - & lepost.fr - \\
\hline \multicolumn{3}{|c|}{ Hyperliens croisant des points de vue 20 (9 \%) } \\
\hline lexpress.fr 12 (11\%) & marianne 5 (6 \%) & lepost.fr $3(8,5 \%)$ \\
\hline \multicolumn{3}{|c|}{ Hyperliens réinterprétants 7 (3\%) } \\
\hline lexpress.fr 2 (2 \%) & marianne2.fr 4 (5 \%) & lepost.fr $1(3 \%)$ \\
\hline
\end{tabular}

Les figures de la lecture dialogique, préfigurant un lecteur prêt à s'engager dans une mise en perspective des arguments entre texte géniteur et texte relié, s'élèvent à $29 \%$ (voir le tableau ci-dessus). Les hyperliens qui déplacent le focus du texte géniteur sans pour autant présenter des contre-arguments, sont certes les plus fréquents (19\%). $11 \%$ des hyperliens dans lexpress.fr vont néanmoins jusqu'à proposer un croisement de points de vue divergents : l'interprétation du réel proposée dans le texte géniteur peut, dans ces cas, se trouver fortement déstabilisée par la lecture du texte relié. Les trois médias présument ainsi, chez leur lectorat, l'aptitude à assumer un certain niveau d'incertitude sur le monde.

Les formes-modèles plutôt pro-extensives et pro-interventives primant dans lexpress.fr n'encouragent pas ces pratiques de lecture attentives et réflexives stipulées par les figures de la lecture dialogique. Des lieux d'indétermination potentiels s'ouvrent ainsi entre les figures de la lecture et les formes-modèles de la page-écran. 
Tableau 8. Figures de la lecture déviative $5 \%$

\begin{tabular}{|l|l|l|} 
Hyperliens déformatoires 7 (4\%) & \\
lexpress.fr 3 $(2,5 \%)$ & marianne2.fr 1 (3,5\%) & lepost.fr 3 (8,5\%) \\
Hyperliens ironisants 3 (1 \%) & lepost.fr 1 (3\%) \\
lexpress.fr 1 (1\%) & marianne2.fr 1 (1\%) & \\
Hyperliens métaphorisants - & lepost.fr - \\
lexpress.fr - & marianne2.fr - & \\
Kiné-trope 1 (0,5 \%) & & lepost.fr 1 (3\%) \\
lexpress.fr - & &
\end{tabular}

Les figures de la lecture déviative sont peu fréquentes (5\%, voir le tableau ci-dessus). Lepost.fr comme marianne2.fr expérimentent des hyperliens ironisants qui présupposent une forte complicité entre lecteur et auteur. Dans les trois médias, certains hyperliens mobilisent aussi un potentiel d'action déformatoire, où les interprétations du réel proposées dans le texte relié se trouvent en forte contradiction avec les arguments avancés dans le texte géniteur. Seul lepost.fr ose le kiné-trope jouant sur l'oscillation entre immersion et distance critique.

Tableau 9. Figures de la lecture immersive 2 (1\%)

Kiné-gramme $1(0,5 \%)$ 
Comme je l'ai déjà indiqué plus haut, les figures de la lecture immersive, préfigurant un lecteur prêt à abandonner au moins partiellement son incrédulité face aux pouvoirs de représentation du texte, sont également rares dans le corpus étudié (voir le tableau ci-dessus). Seul lexpress.fr expérimente le kiné-gramme.

\section{L'hyperlien comme préfiguration d'une relation sociale entre auteurs et médias}

L'hyperlien n'établit pas seulement une relation entre deux textes. Émergent aussi les préfigurations d'une relation sociale entre auteurs et médias, qui est annoncée de façon plus ou moins explicite dans le texte géniteur.

\section{Hyperliens « intro-projectifs »}

Dans ses recommandations aux stagiaires de lexpress.fr, Éric Mettout [2010] avance que les hyperliens doivent certes " pointer vers des articles du site » du groupe, mais qu'ils peuvent aussi, "sans hésitation ", renvoyer à d'autres médias, même si ceux-ci se trouvent en concurrence avec lexpress.fr : "C'est une règle fondamentale sur le Web : plus vous encouragez vos internautes à sortir de votre site, plus ils y reviendront », commente le rédacteur en chef. Dans le corpus d'articles sélectionnés pour cette étude, lexpress.fr est néanmoins celui qui travaille le moins avec des hyperliens externes. Le tableau ci-contre permet de constater que lexpress.fr utilise majoritairement $(67,3 \%)$ des hyperliens internes sans mentionner la source du texte relié dans le texte géniteur. La stratégie de l'hyperlien interne se reflète aussi dans les fonctionnalités du système de gestion de contenu de lexpress.fr : les liens aux pages internes du magazine sont en effet encouragés par la proposition de titres d'articles déjà publiés par le même média.

Les spécificités du système de gestion de contenu ne suffisent cependant pas pour expliquer la fréquence de cette pratique de l'hyperlien que je propose d'appeler " intro-projective ». Alors que dans marianne2. fr comme dans lexpress.fr, l'insertion d'hyperliens internes est favorisée par l'outil, marianne2.fr travaille nettement plus souvent (77,9\%) avec l'hyperlien externe. L'idée de ne pas renvoyer à un média externe qui pourrait favoriser le référencement du site potentiellement concurrentiel s'impose pourtant sans doute avec plus de vigueur dans un média comme 
lexpress.fr, qui accueille encore majoritairement des textes écrits par des journalistes salariés du groupe - auteurs qui, du coup, se rencontrent, se croisent et communiquent sur leur lieu de travail. Lepost.fr se caractérise également par une petite majorité d'hyperliens internes sans indication de la source (44,8\%), pourtant beaucoup moins flagrante.

Dans les trois médias, les journalistes n'évoquent - à une exception près (voir réflexions plus haut sur le rapport entre un journaliste et le rédacteur en chef) - jamais nominativement un confrère dans le texte géniteur lorsqu'ils renvoient à un article interne : entre collègues, la pratique de l'hyperlien intro-projectif semble aller de soi.

Chez le lecteur, ces hyperliens suscitent potentiellement l'impression de traverser un texte écrit par un seul Auteur, dont les identités multiples disparaissent derrière le label du média. Les hyperliens intro-projectifs pourraient par ailleurs être interprétés comme le reflet d'une politique d'entreprise repliée sur ses propres acquis, ne faisant appel au texte de l'autre qu'en cas de nécessité absolue, ou pour se dédouaner. L'étude exploratoire menée auprès des étudiants de l'université Paris 8 (voir chapitre II) a montré que certains lecteurs sont conscients de l'impact de l'hyperlien interne sur le référencement d'un site, et ne sont pas dupes des enjeux économiques de cette pratique.

Dans son livre Ergonomie Web, Amélie Boucher [2011, 27] avance : «Si votre internaute a l'impression de réfléchir et doute de l'issue de son clic, vous avez perdu la première partie de la bataille ». L'une des aides d'orientation que l'Auteur peut adresser à son lecteur est l'indication de la source du texte relié. Dans les 25 articles du corpus, la source d'un texte relié interne au média n'est pourtant jamais indiquée. Si cette pratique peut s'inscrire dans la représentation intro-projective d'un média valorisant ses propres ressources, l'absence fréquente de l'indication de la source dans le cas de l'hyperlien externe peut surprendre.

Or, d'une part l'évocation explicite d'un média concurrentiel peut aller à l'encontre des intérêts économiques du groupe. D’autre part, l'omission de l'indication de la source peut permettre de préserver une part de suspense et de surprise (jeu avec les attentes du lecteur qui fait partie du charme de l'hyperlien), éviter à l'Auteur de prendre explicitement position vis-à-vis du texte relié, et soutenir un certain niveau d'incertitude concernant l'interprétation du monde. 


\section{Hyperlien « extro-projectif »}

Lepost.fr a depuis son lancement donné une place importante aux contributions rédigées par des chroniqueurs non professionnels. Comme les blogueurs invités de marianne2.fr, ces rédacteurs non salariés sont sans doute moins intéressés par l'idée de favoriser le référencement des pages du site par des hyperliens internes. L'hyperlien externe avec indication de la source est également assez fréquent dans lepost.fr (33,6\%). Le lecteur pourrait en déduire que le média fait passer ses propres intérêts économiques derrière la volonté d'informer le lecteur, même si les hyperliens externes ne convoquent pas tous des médias concurrentiels (certains renvoient vers des sites institutionnels, des encyclopédies, etc.). Lepost.fr s'est néanmoins protégé contre les conséquences de cette pratique fréquente de l'hyperlien « extro-projectif » en l'affectant de la balise nofollow. Cette balise, insérée dans le code informatique de la page-écran, indique aux moteurs de recherche que l'hyperlien ne doit pas être valorisé dans le référencement. Dans les faits, elle permet de contourner la prescription technologique selon laquelle tout hyperlien constitue un "vote » positif pour le site relié [Cardon, 2009].

Dans marianne2.fr, la présence majoritaire (67,1\%) d'hyperliens externes sans indication de la source peut en revanche être interprétée comme une ouverture pleinement assumée vers l'autre texte, car ces hyperliens ne sont pas affectés de la balise nofollow. Afin de cerner les enjeux communicationnels d'un texte numérique, il peut donc s'avérer important de lire le code d'une page-écran, et d'examiner quelles représentations s'y logent.

\section{Proposition d'analyse d'un article journalistique}

Avec l'aide des outils d'analyse présentés dans les pages précédentes, le potentiel d'action d'un article journalistique en ligne avec ses hyperliens peut être circonscrit avec précision. Ma méthodologie permet aussi de localiser des lieux d'indétermination qui surgissent potentiellement entre textes géniteurs, textes reliés et formes-modèles, et d'élaborer des interprétations possibles. Comme toute démarche d'interprétation est tributaire d'un point de vue, les hypothèses doivent être clairement explicitées.

Je propose ci-contre l'exemple d'une analyse intégrale de l'article « 22 » du corpus. L'article s'intitule « Face aux crises migratoires, Bruxelles est dans les choux », et a été publié dans marianne2.fr le 2 mars 2011. 
Voici d'abord le texte géniteur avec ses hyperliens, mis en évidence par des soulignements. Deux captures d'écran montrent comment l'article se présente sur la page-écran :

\section{Encadré 2. Face aux crises migratoires, Bruxelles est dans les choux}

L'immigration n'est pas qu'un problème national, elle peut concerner tout un continent, la crise de Lampedusa en est l'exemple. Des milliers de migrants tunisiens voguent dans les eaux internationales puis échouent en Italie en espérant atteindre la France. De même, la frontière gréco-turque où passent notamment des migrants afghans est aussi un lieu de tension. Ces derniers cherchent à aller en Angleterre notamment via la France et son tunnel sous la Manche. Sans parler des enclaves espagnoles de Ceuta et Melilla, situées au Maroc.

Vouloir créer des frontières communes tout en abolissant les frontières intérieures a naturellement des conséquences sur les migrations. En franchissant les frontières de l'Europe, les immigrants espèrent pouvoir circuler librement sur le continent. $\mathrm{Vu}$ que la question migratoire s'impose désormais à tous, le bon sens imposerait donc que l'on mette en place une gestion commune des frontières. Ce n'est pourtant pas encore le cas. La crise de Lampedusa a d'ailleurs donné un bel exemple de cette désunion européenne. L'Italie avait accusé Bruxelles de ne pas lui apporter d'aide. Bruxelles a rétorqué que c'était Rome qui avait refusé la main tendue.
Toutefois, un outil a pu faire son apparition dans cette crise migratoire : l'agence Frontex. Un nom très novlangue pour l'Agence européenne pour la gestion de la coopération opérationnelle aux frontières extérieures. Créée en 2004, opérationnelle depuis 2005, elle a notamment trois missions : étudier les mouvements migratoires, coordonner des opérations de gestion de flux migratoire entre États membres et permettre des vols communs de retour vers les pays d'origine des migrants refoulés. Son budget pour 2011 dépasse les 88 millions d'euros. Elle a mené 43 opérations de contrôle ou de vols retours en 2010 selon des chiffres des eurodéputés Europe Écologie, un chiffre qui paraît faible vu l'enjeu.

L'agence Frontex possède aussi un bras armé avec les Rabits (pour Rapid intervention border teams, admirez l'humour bruxellois). Ce sont des policiers et autres agents armés chargés d'intervenir rapidement à la demande d'un État membre pour gérer un problème migratoire. Ils gardent leurs uniformes nationaux mais ont un brassard de l'UE et sont placés sous le commandement de l'État demandeur. Ce n'est donc pas à proprement parler une police européenne des frontières. Ces équipes sont notamment intervenues en Grèce à l'automne 
dernier face à un afflux de migrants venant de la frontière avec la Turquie.

Dans la crise de Lampedusa, Frontex a déployé un autre outil en dépêchant des « experts » sur place dans le cadre de l'opération Hermès. Aux alentours de 50 personnels issus de différents pays européens (France, Portugal, Danemark...) sont venus prêter main forte à l'Italie (qui dirige la mission). Ces experts seront notamment chargés de l'accueil et de l'interrogatoire des migrants. Ils ne seront pas seulement policiers mais aussi spécialistes des " relations interculturelles » ou du droit d'asile, comme l'a précisé la Commission européenne à marianne2. Une mise à disposition de moyens aériens en vue de la surveillance des frontières est également prévue. L'opération devrait être terminée le 31 mars selon la Commission. Mais Bruxelles n'a pas su nous dire si d'autres moyens sont prévus : « On suit les évolutions au jour le jour ».

C'est justement le problème. L'agence Frontex apparaît comme le symbole d'une Europe dépassée par la mondialisation des échanges. Elle peine à s'imposer comme outil commun de régulation et reste suspendue au bon vouloir des États membres qui mettent à disposition troupes et matériel. « On reçoit une demande d'aide d'un État membre et chaque État dit quels moyens il peut offrir », nous explique la Commission. En matière de migrations, c'est donc le règne du chacun pour soi. «Ce qui est en cause, c'est que l'agence Frontex doit être assurée que les États membres fournissent les moyens promis ", ajoute l'eurodéputé PS Sylvie Guillaume, contactée par marianne2. Mais créer une véritable police aux frontières européenne, idée qui est toujours sur la table, empiéterait sur la souveraineté des États. D'un autre côté, sans instruments politiques adéquats, la mise en place de frontière commune est ingérable.

\section{Chacun pour soi.}

Tout le dilemme de la construction européenne est là. On a voulu inscrire l'Europe dans la mondialisation, abolir les frontières, sans voir que la mondialisation doit aussi être régulée pour être viable, que l'on parle d'économie ou de migrations. Les règles européennes, au lieu de donner un cadre commun sûr, renvoient au chacun pour soi. Une chose qui ne protège ni les États ni les migrants. Mais il n'y a pas que l'aspect répressif qui compte. En matière d'asile par exemple, elles imposent que le migrant fasse sa demande uniquement dans le pays où il est arrivé en premier (ici l'Italie). Si un autre État l'arrête, il est alors renvoyé dans le pays d'entrée. La base de données Eurodac (qui recense les empreintes digitales) permet d'ailleurs de repérer les demandeurs multiples.

Cette règle a un effet pervers : faire porter une large part des politiques migratoires sur les pays ayant une frontière exposée, qui deviennent ainsi un guichet géant pour tous les demandeurs d'asile. Un petit État comme Malte a du mal à faire face. La Grèce a également menacé, avec le soutien de Paris, de construire une clôture à sa frontière turque, s'estimant trop isolée face à 
l'immigration clandestine. Bruxelles s'est opposé au projet, pourtant, à Ceuta et Melilla, l'UE a financé la construction de barrières de ce type. Mais les renvois vers la Grèce posent un autre problème étant contestés par la Cour européenne des droits de l'homme, car Athènes ne respecte pas assez les droits des demandeurs d'asile. L'Italie souhaite que le sujet de migrations soit à l'ordre du jour du prochain sommet européen. La crise de Lampedusa pourrait faire bouger les lignes vers plus de solidarité mais le chemin est encore long. " La très grande liberté laissée aux États aboutit à des systèmes hétérogènes », déplore Sylvie Guillaume. Une solution intermédiaire pourrait alors être des accords d'État à État sans passer par Bruxelles, solution écartée par l'eurodéputé. Pour elle, tous les pays de l'UE doivent être impliqués : "Les Suédois sont aussi concernés par ce qu'il se passe en Méditerranée ». Mais pour l'instant, la solidarité européenne n'est pas de mise. Comme si l'UE était seulement vouée à être le cadre d'une " concurrence libre et non faussée » et non celui d'une protection, laissant les États les plus faibles, comme la Grèce, en première ligne. Bien sûr, il reste la solution de confier la surveillance de nos frontières à la Libye de Kadhafi.

\section{Quelle politique d'asile?}

$\mathrm{Au}$ cœur donc de la crise de Lampedusa, c'est donc bien le degré de souveraineté que sont prêts à abandonner les États qui est mis en débat. Ce qui est politiquement difficile à faire avaler. Contrairement donc au discours ambiant, l'abolition des souverainetés est plus un vœu pieux qu'un horizon inéluctable. Si la politique d'asile en Europe est en cours de refonte, pour l'instant, « rien n'est commun » à ce sujet parmi les États membres, précise Sylvie Guillaume. Réguler n'est décidément pas le fort de l'UE. Car, au-delà de la question de Frontex, la politique migratoire de l'Union manque encore d'instruments communs. "Si les États membres veulent gérer le rapport migratoire avec la seule agence Frontex, c'est la mauvaise façon de faire ", estime Sylvie Guillaume. L'eurodéputé socialiste plaide pour " un dispositif d'asile commun ».

Ce serait un minimum, une Europe ouverte ne signifie pas forcément un monde sans règles. Il reste à savoir si les États membres sont capables d'accepter des règles communes en ce domaine. Pour l'instant, en France, on loue la nécessité d'une véritable politique européenne d'immigration. Le Quai d'Orsay avait déjà appelé au « renforcement opérationnel de Frontex ». Lundi, François Fillon a réaffirmé que la solution face aux crises migratoires était " une réponse collective, une réponse européenne sur ce sujet, d'abord pour faire en sorte qu'il y ait le moins de flux migratoires possibles, et ensuite pour accueillir les personnes qu'il faudra accueillir ». Le contexte politique imposait un tel discours. Mais la géographie qui sépare la Finlande de l'Italie, peut-elle permettre une position commune sur la crise migratoire méditerranéenne? 
Figure 13. Captures d'écran d'extraits de l'article (22) paru sur marianne2.fr
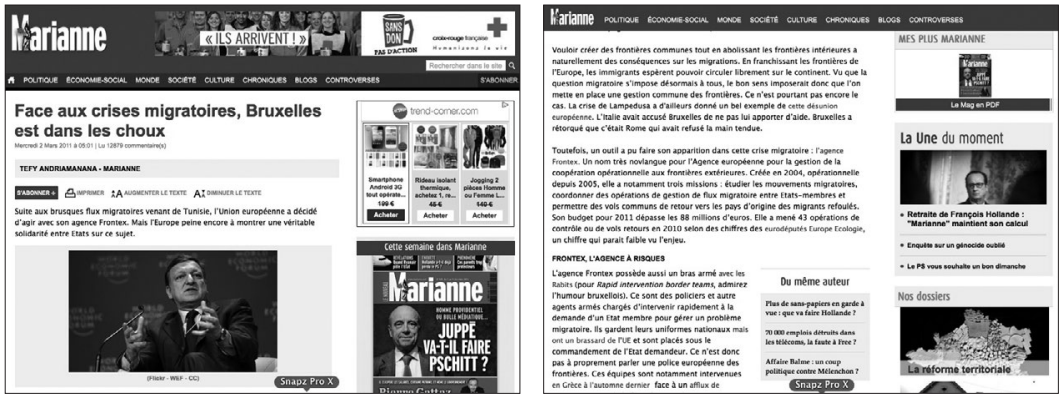

Pour réaliser cette analyse, il s'agit d'abord de circonscrire le texte à lire. Il serait tout à fait possible de proposer une analyse qui prendrait en compte les répertoires et stratégies de l'article géniteur, ceux du texte relié au premier hyperlien de cet article, ceux du texte relié au premier hyperlien dans ce nouveau texte relié, et ainsi de suite. Ce n'est pas le choix que j'ai fait lorsque j'ai analysé les 25 articles du corpus, dont l'article 22. Afin de réaliser une analyse aussi exhaustive que possible d'un article en tant que texte géniteur, j'ai décidé de prendre en compte tous les textes reliés aux hyperliens insérés dans cet article, sans me pencher sur les hyperliens présents à leur tour dans les textes reliés.

Après la définition du périmètre de lecture, j'ai identifié, paragraphe par paragraphe, les répertoires du texte géniteur : j'ai donc circonscrit les savoirs, compétences et normes auxquels le texte fait appel. Pour chaque hyperlien, j'ai ensuite dressé un catalogue d'attentes possibles en relevant d'une part les éventuelles annonces concernant le texte relié, et en prenant d'autre part en compte les figurations les plus courantes de l'hyperlien et du texte journalistique. Pour assurer la faisabilité du projet, j'ai décidé de regrouper ces attentes potentielles autour des concepts clés « informationnel » et "dialogique ». Ces deux termes ne désignent évidemment que des tendances : les attentes d'un lecteur peuvent être à la fois informationnelles et dialogiques ou ni l'une ni l'autre, variant en fonction du dispositif (lecture sur une liseuse, un ordinateur fixe, dans le train ou sur le canapé, etc.) et d'autres facteurs contextuels.

Le tableau suivant résume les répertoires et stratégies du texte géniteur, et liste des attentes possibles vis-à-vis du texte relié dans l'exemple choisi. Je présente cette étape de façon exhaustive pour que le lecteur de ce livre puisse comprendre tous les détails de ma démarche. 


\section{Répertoires, stratégies, annonces et reprises dans le texte géniteur}

Tableau 10. Article 22 http://www.marianne2.fr/Face-aux-crisesmigratoires-Bruxelles-est-dans-les-choux_a203368.html

\begin{tabular}{|c|c|}
\hline Titre & $\begin{array}{l}\text { «Face aux crises migratoires, Bruxelles est dans les choux ». } \\
\text { Répertoires : relations Bruxelles - UE supposées connues ; } \\
\text { expression anticipée comme familière : « être dans les choux ». }\end{array}$ \\
\hline Chapeau & $\begin{array}{l}\text { Suite aux flux migratoires venant de Tunisie, l'UE a décidé } \\
\text { de mobiliser son agence Frontex. Mais les États membres ne } \\
\text { font pas preuve de solidarité. } \\
\text { Répertoires : font référence à l'importance et à la cause des } \\
\text { flux migratoires venant de Tunisie en } 2011 \text {. }\end{array}$ \\
\hline Paragraphe 1 & $\begin{array}{l}\text { "L'immigration n'est pas qu'un problème national, elle peut } \\
\text { concerner tout un continent, la crise de Lampedusa en est } \\
\text { l'exemple " (1). Des milliers de Tunisiens espèrent atteindre } \\
\text { la France via l'Italie. Des migrants afghans tentent de passer } \\
\text { la frontière entre la Grèce et la Turquie. Ceuta et Melilla au } \\
\text { Maroc accueillent également des migrants. } \\
\text { Répertoires : renvoient aux causes de la migration } \\
\text { tunisienne; la situation géographique de Lampedusa, Ceuta } \\
\text { et Melilla est supposée connue. }\end{array}$ \\
\hline $\begin{array}{l}\text { Attentes potentielles } \\
\text { hyperlien } 1\end{array}$ & $\begin{array}{l}\text {-informationnelles : la crise de Lampedusa est citée comme } \\
\text { " exemple » dans le texte géniteur : les attentes sont } \\
\text { potentiellement orientées vers des images documentant la } \\
\text { situation, ou d'autres exemples de crises. } \\
\text { - dialogiques: le lecteur pourrait aussi s'attendre à des points } \\
\text { de vue divergents sur cette crise, par exemple les avis des } \\
\text { migrants ; mais aucune annonce d'un tel changement de } \\
\text { focus n'est faite dans le texte géniteur. }\end{array}$ \\
\hline Paragraphe 2 & $\begin{array}{l}\text { L'abolition des frontières en Europe a des conséquences } \\
\text { pour tous les pays européens. Or, l'UE tarde à mettre en place } \\
\text { une gestion des frontières commune. La crise de Lampedusa } \\
\text { est un exemple de "cette désunion européenne » (2). L'Italie } \\
\text { et Bruxelles s'accusent mutuellement. } \\
\text { Répertoires : font référence à l'histoire de l'UE, et aux conflits } \\
\text { récents entre pays membres concernant les migrations. }\end{array}$ \\
\hline
\end{tabular}




\begin{tabular}{|c|c|}
\hline $\begin{array}{l}\text { Attentes potentielles } \\
\text { hyperlien } 2\end{array}$ & $\begin{array}{l}\text {-informationnelles : le texte relié citera peut-être un } \\
\text { exemple de la " désunion européenne ». Les attentes } \\
\text { sont potentiellement orientées vers des compléments } \\
\text { d'information concernant cette désunion. } \\
\text { - dialogiques: des points de vue de différents pays pourraient } \\
\text { être juxtaposés; mais aucune annonce d'une telle relation } \\
\text { dialogique n'est faite dans le texte géniteur. }\end{array}$ \\
\hline Paragraphe 3 & $\begin{array}{l}\text { Un outil commun de l'UE est "l'agence Frontex » (3). Doté } \\
\text { d'un budget de } 88 \text { millions d'euros en 2011, elle étudie } \\
\text { les mouvements migratoires, coordonne des opérations } \\
\text { de gestion de flux, gère les vols de retour des migrants } \\
\text { refoulés. En } 2010 \text {, elle a mené } 43 \text { opérations de contrôle ou } \\
\text { de vols retours selon les " eurodéputés Europe Écologie " } \\
\text { (4); chiffre que l'auteur considère comme faible. } \\
\text { Répertoires : pratiques de refoulement de l'UE, ainsi que le } \\
\text { parti politique " Europe Écologie » sont supposés connus } \\
\text { par le lecteur. }\end{array}$ \\
\hline $\begin{array}{c}\text { Attentes potentielles } \\
\text { hyperlien } 3\end{array}$ & $\begin{array}{l}\text {-informationnelles : si le lecteur active l'hyperlien posé sur } \\
\text { "l'agence Frontex » avant la lecture de la suite, il s'attend } \\
\text { peut-être à une définition de l'agence, des précisions sur } \\
\text { sa mission. S'il lit les informations intégrées dans le texte } \\
\text { géniteur avant de cliquer, il pourrait s'attendre plutôt à } \\
\text { accéder aux sources de ces informations. } \\
\text { - dialogiques : un débat autour de l'agence Frontex pourrait } \\
\text { être proposé ; mais un tel débat n'est pas annoncé dans le } \\
\text { texte géniteur. }\end{array}$ \\
\hline $\begin{array}{l}\text { Attentes potentielles } \\
\text { hyperlien } 4\end{array}$ & $\begin{array}{l}\text { - informationnelles : l'hyperlien sur " eurodéputés Europe } \\
\text { Écologie » pourrait renvoyer à la source des chiffres cités } \\
\text { sur le site du parti. } \\
\text { • dialogiques : une prise de position critique vis-à-vis de } \\
\text { Frontex s'annonce dans le texte géniteur, qui pourrait } \\
\text { être développée par le texte relié via une juxtaposition de } \\
\text { points de vue. }\end{array}$ \\
\hline Paragraphe 4 & $\begin{array}{l}\text { L'auteur approfondit la description des missions de Frontex. } \\
\text { "Avec les Rabits » (5) (Rapid intervention border teams - } \\
\text { l'auteur fustige " l'humour " du choix de l'appellation), } \\
\text { Frontex possède un " bras armé ». La troupe intervient } \\
\text { à la demande d'un État membre. Les soldats gardent les } \\
\text { uniformes nationaux " mais ont un brassard de l'UE » (6). Ils } \\
\text { sont intervenus " en Grèce à l'automne dernier » (7), face à } \\
\text { un " afflux de migrants » (8) venant de la frontière turque ». } \\
\text { Répertoires: avec sa remarque ironique concernantl'appellation } \\
\text { de la troupe, l'auteur anticipe sur la complicité du lecteur. }\end{array}$ \\
\hline
\end{tabular}




\begin{tabular}{|c|c|}
\hline $\begin{array}{l}\text { Attentes potentielles } \\
\text { hyperlien } 5\end{array}$ & $\begin{array}{l}\text { - informationnelles : si le lecteur active l'hyperlien sur } \\
\text { "Avec les Rabits » avant de continuer la lecture du } \\
\text { paragraphe, il s'attend peut-être à une définition, ou alors } \\
\text { à un renvoi vers la source des informations. } \\
\text { - dialogiques : compte tenu de la remarque de l'auteur } \\
\text { concernant le choix de l'appellation, le lecteur pourrait } \\
\text { s'attendre à des prises de position critiques envers la } \\
\text { troupe, voire à un affichage de caricatures. }\end{array}$ \\
\hline $\begin{array}{l}\text { Attentes potentielles } \\
\text { hyperlien } 6\end{array}$ & $\begin{array}{l}\text { - informationnelles : le lecteur s'attend peut-être à la photo } \\
\text { d'un uniforme avec le brassard en activant l'hyperlien sur } \\
\text { «mais ont un brassard de l'UE ». } \\
\text { - dialogiques : les éventuelles tensions liées à la double } \\
\text { appartenance des soldats pourraient être exposées et } \\
\text { problématisées. }\end{array}$ \\
\hline $\begin{array}{l}\text { Attentes potentielles } \\
\text { hyperlien } 7\end{array}$ & $\begin{array}{l}\text { - informationnelles : le lecteur s'attend peut-être à des } \\
\text { précisions sur la situation en Grèce en activant l'hyperlien } \\
\text { sur « en Grèce à l'automne dernier ». } \\
\text { - dialogiques : différentes opinions sur cette intervention } \\
\text { pourraient se croiser grâce au texte relié ; mais pas } \\
\text { d'annonce d'un tel potentiel d'action dialogique dans le } \\
\text { texte géniteur. }\end{array}$ \\
\hline $\begin{array}{l}\text { Attentes potentielles } \\
\text { hyperlien } 8\end{array}$ & $\begin{array}{l}\text { - informationnelles : face à l'hyperlien sur « afflux de } \\
\text { migrants ", le lecteur pourrait s'attendre à des photos ou } \\
\text { vidéos montrant cet afflux, ou à des précisions sur leur } \\
\text { provenance. } \\
\text { - dialogiques : un changement de perspective pourrait } \\
\text { permettre au lecteur de découvrir par exemple le point } \\
\text { de vue des migrants; mais un tel changement n'est pas } \\
\text { annoncé dans le texte géniteur. }\end{array}$ \\
\hline Paragraphe 5 & $\begin{array}{l}\text { L'auteur revient vers Lampedusa et un autre outil de l'agence } \\
\text { Frontex : l'équipe d'« experts " déployée " dans le cadre } \\
\text { de l'opération Hermès » (9). Ces experts sont " chargés de } \\
\text { l'accueil et de l'interrogatoire des migrants " (10). Selon } \\
\text { la Commission européenne, ils ne sont pas seulement } \\
\text { policiers, mais spécialistes des relations interculturelles ou } \\
\text { du droit d'asile. Une mise à disposition de moyens aériens } \\
\text { est prévue. Bruxelles suit les évolutions " au jour le jour ». } \\
\text { Répertoires : le texte anticipe sur les connaissances } \\
\text { du lecteur concernant le statut et les fonctions de la } \\
\text { Commission européenne. }\end{array}$ \\
\hline
\end{tabular}




\begin{tabular}{|c|c|}
\hline $\begin{array}{l}\text { Attentes potentielles } \\
\text { hyperlien } 9\end{array}$ & $\begin{array}{l}\text { - informationnelles : en activant l'hyperlien sur " dans } \\
\text { le cadre de l'opération Hermès ", le lecteur pourrait } \\
\text { s'attendre à une description plus détaillée, des images ou } \\
\text { vidéos de l'opération Hermès. } \\
\text { - dialogiques : il pourrait éventuellement s'attendre à } \\
\text { découvrir des prises de position critiques vis-à-vis de } \\
\text { l'opération, même si aucune annonce dans le texte } \\
\text { géniteur ne le laisse supposer. }\end{array}$ \\
\hline $\begin{array}{l}\text { Attentes potentielles } \\
\text { hyperlien } 10\end{array}$ & $\begin{array}{l}\text { - informationnelles : le texte relié à " l'accueil et de } \\
\text { l'interrogatoire des migrants " pourrait proposer des } \\
\text { descriptions plus précises de la mission, des exemples } \\
\text { d'interrogatoires, des vidéos et photos. } \\
\text { - dialogiques : le lecteur pourrait s'attendre à un point } \\
\text { de vue critique sur ces pratiques, par exemple à travers } \\
\text { l'interview d'un migrant ; mais un tel changement de point } \\
\text { de vue n'est pas annoncé dans le texte géniteur. }\end{array}$ \\
\hline Paragraphe 6 & $\begin{array}{l}\text { Le journaliste revient à son argumentation principale } \\
\text { esquissée à la fin du paragraphe précédent : l'Europe lui } \\
\text { paraît dépassée par la mondialisation des échanges. Les } \\
\text { actions de Frontex restent dépendantes du bon vouloir } \\
\text { des États membres. L'eurodéputé PS " Sylvie Guillaume " } \\
\text { (11) confirme que Frontex doit s'assurer que les États } \\
\text { fournissent les moyens promis. Une police européenne des } \\
\text { frontières mettrait en cause la souveraineté des États, mais } \\
\text { semble nécessaire. } \\
\text { Répertoires : l'auteur renvoie à la constitution de l'UE, ainsi } \\
\text { qu'au rôle de la France. }\end{array}$ \\
\hline $\begin{array}{l}\text { Attentes potentielles } \\
\text { hyperlien } 11\end{array}$ & $\begin{array}{l}\text { - informationnelles : le lecteur s'attend potentiellement à } \\
\text { un CV, à la page personnelle de Sylvie Guillaume ; ou à } \\
\text { l'affichage de l'interview complète. } \\
\text { - dialogiques : compte tenu du texte géniteur, un renvoi } \\
\text { dialogique vers des acteurs contestant le point de vue de } \\
\text { Sylvie Guillaume paraît certes peu probable ; le lecteur } \\
\text { pourrait néanmoins s'attendre à un déplacement de focus. }\end{array}$ \\
\hline
\end{tabular}




\begin{tabular}{|c|c|}
\hline Paragraphe 7 & $\begin{array}{l}\text { L'auteur approfondit l'analyse du " dilemme " de l'UE : } \\
\text { l'ouverture des frontières inscrit l'Europe dans la } \\
\text { mondialisation tout en exigeant une régulation. Or, les règles } \\
\text { renvoient au " chacun pour soi ". En matière d'asile, les } \\
\text { migrants doivent faire la demande dans le pays où ils entrent } \\
\text { en premier. S'ils sont arrêtés ailleurs, ils sont renvoyés vers } \\
\text { le pays d'entrée. La base de données «Eurodac " (12) permet } \\
\text { de repérer les demandes d'asile multiples. } \\
\text { Répertoires : les processus de la mondialisation et sa } \\
\text { relation avec l'ouverture des frontières sont supposés } \\
\text { connus par le lecteur. }\end{array}$ \\
\hline $\begin{array}{l}\text { Attentes potentielles } \\
\text { hyperlien } 12\end{array}$ & $\begin{array}{l}\text { - informationnelles : le lecteur pourrait s'attendre à } \\
\text { une définition de la base Eurodac, et à des explications } \\
\text { concernant son cadre juridique. } \\
\text { - dialogiques : le texte relié pourrait donner accès au point } \\
\text { de vue des migrants, leurs motivations pour déposer des } \\
\text { demandes d'asile multiples ; mais un tel changement de } \\
\text { perspective n'est pas annoncé dans le texte géniteur. }\end{array}$ \\
\hline Paragraphe 8 & $\begin{array}{l}\text { L'auteur explicite les conséquences de cette règle d'asile : } \\
\text { les pays avec une frontière " exposée " sont confrontés à } \\
\text { un afflux particulièrement important de migrants. Un petit } \\
\text { État " comme Malte a du mal à faire face " (13). La Grèce } \\
\text { a " également menacé » (14), avec le " soutien de Paris " } \\
\text { (15), d'ériger une clôture à sa frontière turque. L'UE s'est } \\
\text { opposée au projet, tout en finançant la construction de } \\
\text { clôtures à Ceuta et Melilla. Les renvois de migrants vers la } \\
\text { Grèce sont contestés " par la Cour européenne des droits } \\
\text { de l'homme " (16) parce que le pays ne respecte pas assez } \\
\text { les droits des migrants. Même si l'Italie a demandé que le } \\
\text { sujet des migrations soit mis à l'ordre du jour du prochain } \\
\text { sommet européen, le chemin vers plus de solidarité paraît } \\
\text { encore long. } \\
\text { Répertoires : la situation géographique de Malte, Ceuta et } \\
\text { Melilla, ainsi que les missions de la Cour européenne des } \\
\text { droits de l'homme sont supposées connues. }\end{array}$ \\
\hline $\begin{array}{l}\text { Attentes potentielles } \\
\text { hyperlien } 13\end{array}$ & $\begin{array}{l}\text { - informationnelles : suite à l'activation de l'hyperlien sur } \\
\text { "comme Malte a du mal à faire face ", le lecteur pourrait } \\
\text { s'attendre à des compléments d'information sur la situation } \\
\text { à Malte, des photos et vidéos. } \\
\text { - dialogiques : une contestation de l'énoncé que " Malte a } \\
\text { du mal à faire face " paraît peu probable compte tenu des } \\
\text { répertoires du texte géniteur ; un déplacement de focus } \\
\text { pourrait néanmoins être attendu. }\end{array}$ \\
\hline
\end{tabular}




\begin{tabular}{|c|c|}
\hline $\begin{array}{l}\text { Attentes potentielles } \\
\text { hyperlien } 14\end{array}$ & $\begin{array}{l}\text { - informationnelles : le lecteur pourrait souhaiter des } \\
\text { compléments d'information sur la " menace " prononcée } \\
\text { par la Grèce en cliquant. } \\
\text { - dialogiques : il pourrait s'attendre aussi à des arguments } \\
\text { contre l'installation de la clôture. Comme la suite du } \\
\text { texte géniteur expose l'avis de l'UE, le texte relié pourrait } \\
\text { renvoyer vers cet avis. }\end{array}$ \\
\hline $\begin{array}{l}\text { Attentes potentielles } \\
\text { hyperlien } 15\end{array}$ & $\begin{array}{l}\text { - informationnelles : le lecteur pourrait approcher cet } \\
\text { hyperlien en s'attendant à des compléments d'information } \\
\text { sur le « soutien de Paris », ainsi que sur sa justification. } \\
\text { - dialogiques : le texte relié pourrait donner accès à des avis } \\
\text { contestant ce soutien, en France ou à l'étranger ; compte } \\
\text { tenu de la critique de l'UE exposée dans le texte géniteur, } \\
\text { une telle relation dialogique paraît même assez plausible. }\end{array}$ \\
\hline $\begin{array}{l}\text { Attentes potentielles } \\
\text { hyperlien } 16\end{array}$ & $\begin{array}{l}\text { - informationnelles : en activant l'hyperlien sur «par la Cour } \\
\text { européenne des droits de l'homme », le lecteur pourrait } \\
\text { s'attendre à des explications concernant les fonctions et le } \\
\text { statut de la Cour en général, et sur la position face au projet } \\
\text { grec en particulier. } \\
\text { - dialogiques : compte tenu du texte géniteur, il paraît assez } \\
\text { improbable que le texte relié expose un avis contraire à celui } \\
\text { de la Cour européenne ; le texte relié pourrait néanmoins } \\
\text { donner accès à une perspective différente (par exemple, } \\
\text { celle des habitants). }\end{array}$ \\
\hline Paragraphe 9 & $\begin{array}{l}\text { L'auteur revient vers le dilemme de l'UE face aux } \\
\text { migrations. L'eurodéputé Sylvie Guillaume déplore la grande } \\
\text { hétérogénéité des systèmes. Des accords d'État à État } \\
\text { sont néanmoins rejetés par la député à cause du principe } \\
\text { de solidarité, même si des États comme la Grèce restent } \\
\text { sans protection. "Bien sûr, il reste la solution de confier la } \\
\text { surveillance de nos frontières à la Libye de Kadhafi " (17), } \\
\text { commente l'auteur. } \\
\text { Répertoires: l'auteur présuppose chez le lecteur la capacité } \\
\text { de comprendre l'ironie du commentaire sur la Libye ; une } \\
\text { connaissance de l'histoire récente de la Libye est donc } \\
\text { également anticipée. }\end{array}$ \\
\hline
\end{tabular}




\begin{tabular}{|c|c|}
\hline $\begin{array}{l}\text { Attentes potentielles } \\
\text { hyperlien } 17\end{array}$ & $\begin{array}{l}\text { - informationnelles : le lecteur s'attend potentiellement à } \\
\text { des explications concernant la position libyenne face à la } \\
\text { surveillance des frontières. } \\
\text { - dialogiques/déviatifs : compte tenu du texte géniteur, le } \\
\text { texte relié pourrait faire éclater au grand jour l'absurdité } \\
\text { des propositions de Kadhafi, confirmant le retournement } \\
\text { ironique de l'énoncé dans le texte géniteur. }\end{array}$ \\
\hline Paragraphe 10 & $\begin{array}{l}\text { L'argumentation se recentre autour du degré de souveraineté } \\
\text { que les États membres seraient prêts à abandonner. Pour } \\
\text { l'instant, les États n'ont pas trouvé de dénominateur commun } \\
\text { concernant la politique d'asile. D'après l'eurodéputé Sylvie } \\
\text { Guillaume, l'UE manque d'outils : par exemple, d'un } \\
\text { dispositif d'asile commun. } \\
\text { Répertoires : renvoient au statut de l'UE et de ses États } \\
\text { membres. }\end{array}$ \\
\hline Paragraphe 11 & $\begin{array}{l}\text { Pour l'auteur, un « dispositif d'asile commun » serait } \\
\text { un " minimum ". Il n'est pas sûr que les États membres } \\
\text { arriveront à un consensus. En France, le «Quai d'Orsay » a } \\
\text { souligné la nécessité d'une politique européenne en matière } \\
\text { d'immigration. François Fillon affirme que " la solution face } \\
\text { aux crises migratoires " (18) ne peut être qu’une " réponse } \\
\text { européenne ». Selon l'auteur, la situation politique impose } \\
\text { un tel discours ; il se montre néanmoins sceptique par } \\
\text { rapport aux possibilités de trouver rapidement des solutions } \\
\text { concrètes. } \\
\text { Répertoires : la localisation d'un ministère au Quai d'Orsay } \\
\text { et de ses missions, ainsi que la fonction de François Fillon } \\
\text { sont anticipées comme connues par le lecteur. }\end{array}$ \\
\hline $\begin{array}{l}\text { Attentes potentielles } \\
\text { hyperlien } 18\end{array}$ & $\begin{array}{l}\text { - informationnelles : le texte relié pourrait renvoyer à } \\
\text { la source du discours de François Fillon, ou fournir des } \\
\text { compléments d'information. } \\
\text { - dialogiques : la position de François Fillon pourrait se } \\
\text { trouver contestée par le texte relié ; en cohérence avec le } \\
\text { commentaire critique de l'auteur, des opinions divergentes } \\
\text { pourraient être exposées. }\end{array}$ \\
\hline
\end{tabular}

Après cette phase d'analyse, les attentes potentielles envers les hyperliens ont été mises en perspective avec les répertoires de chaque texte relié. Le potentiel d'action des procédés rhétoriques de l'hyperlien a ainsi pu être circonscrit. Émergent progressivement les figures de la lecture anticipées par l'article. 


\section{Tableau 11. Identification des figures de la lecture}

\begin{tabular}{|c|c|}
\hline $\begin{array}{c}\text { Hyperlien } 1 \\
\text { « la crise de Lampedusa } \\
\frac{\text { en est l'exemple. » }}{\text { potentiel d'action }} \\
\text { d'abord explicatif, ensuite } \\
\text { déplaçant le focus }\end{array}$ & $\begin{array}{l}\text { Le texte relié au texte géniteur par un hyperlien intro- } \\
\text { projectif est rédigé par le même auteur. Il répond } \\
\text { d'abord aux attentes potentiellement informationnelles } \\
\text { du lecteur en fournissant d'autres exemples de crises } \\
\text { migratoires. Ces exemples sont cités dans la suite } \\
\text { du texte géniteur. À la fin du texte relié, le focus se } \\
\text { déplace vers les réactions dans la politique française. } \\
\text { Une critique ferme est avancée vis-à-vis de Nicolas } \\
\text { Sarkozy, critique qui correspond aux figurations } \\
\text { associées au magazine Marianne. }\end{array}$ \\
\hline $\begin{array}{c}\text { Hyperlien } 2 \\
\text { « cette désunion } \\
\text { européenne » } \\
\text { potentiel d'action d'abord } \\
\text { explicatif, déplaçant } \\
\text { ensuite le focus }\end{array}$ & $\begin{array}{l}\text { L'hyperlien renvoie vers le même article que } \\
\text { l'hyperlien précédent. La stratégie et les répertoires } \\
\text { du texte relié répondent potentiellement aux attentes } \\
\text { informationnelles du lecteur ; en revanche, cette } \\
\text { multiplication des hyperliens vers le même texte } \\
\text { relié peut agacer (voir étude exploratoire avec des } \\
\text { étudiants de Paris 8). }\end{array}$ \\
\hline $\begin{array}{l}\text { Hyperlien } 3 \\
\text { « l'agence Frontex } ~ \\
\text { potentiel d'action } \\
\text { définissant }\end{array}$ & $\begin{array}{l}\text { Le texte relié à un hyperlien extro-projectif } \\
\text { sans indication des sources répond aux attentes } \\
\text { potentiellement informationnelles du lecteur en } \\
\text { quête de définitions, renvoyant vers un article de } \\
\text { Wikipédia consacré à Frontex. }\end{array}$ \\
\hline $\begin{array}{l}\text { Hyperlien } 4 \\
\text { « eurodéputés Europe } \\
\text { Écologie " } \\
\text { potentiel d'action croisant } \\
\text { des points de vue }\end{array}$ & $\begin{array}{l}\text { L'hyperlien extro-projectif avec indication des } \\
\text { sources renvoie vers la source des chiffres cités, le } \\
\text { site d'Europe Écologie. Le texte relié va plus loin que } \\
\text { la critique des chiffres : il accuse l'agence Frontex } \\
\text { de violation des droits de l'homme, et illustre ces } \\
\text { propos de photos faisant potentiellement référence } \\
\text { aux camps de concentration. Le texte relié propose } \\
\text { donc un point de vue divergent, mettant en question } \\
\text { certaines affirmations du texte géniteur. }\end{array}$ \\
\hline $\begin{array}{l}\text { Hyperlien } 5 \\
\text { «Avec les Rabits » } \\
\text { potentiel d’action } \\
\text { définissant }\end{array}$ & $\begin{array}{l}\text { Répondant aux attentes informationnelles, l'hyperlien } \\
\text { extro-projectif renvoie vers le site touteleurope.eu, } \\
\text { qui propose une définition de la troupe. }\end{array}$ \\
\hline $\begin{array}{c}\text { Hyperlien } 6 \\
\text { « mais ont un brassard de } \\
\begin{array}{c}\text { l'UE » } \\
\text { potentiel d'action } \\
\text { illustratif }\end{array}\end{array}$ & $\begin{array}{l}\text { Le texte convoqué par un hyperlien extro-projectif } \\
\text { sans indication des sources se trouve sur un site de } \\
\text { l'UE et affiche la photo d'un uniforme de Rabit avec } \\
\text { le brassard. Cette illustration répond potentiellement } \\
\text { aux attentes suscitées par le texte géniteur. }\end{array}$ \\
\hline
\end{tabular}




\begin{tabular}{|c|c|}
\hline $\begin{array}{c}\text { Hyperlien } 7 \\
\text { « en Grèce à l'automne } \\
\begin{array}{c}\text { dernier » } \\
\text { potentiel d'action } \\
\text { explicatif }\end{array}\end{array}$ & $\begin{array}{l}\text { Le texte relié par hyperlien extro-projectif sans } \\
\text { indication des sources sur euranet.eu fournit des } \\
\text { compléments d'information sur la mission de Frontex } \\
\text { en Grèce, confirmant les informations du texte } \\
\text { géniteur sur le ton neutre d'une dépêche. }\end{array}$ \\
\hline $\begin{array}{c}\text { Hyperlien } 8 \\
\text { " afflux de migrants » } \\
\text { potentiel d'action croisant } \\
\text { les points de vue }\end{array}$ & $\begin{array}{l}\text { L'hyperlien extro-projectif sans indication des } \\
\text { sources renvoie vers le site de France } 24 \text {, et plus } \\
\text { précisément vers un reportage (texte et vidéo) } \\
\text { consacré à la situation sur la frontière : d'un côté, } \\
\text { le texte relié pointe la nécessité de gérer l'afflux de } \\
\text { migrants ; d'un autre côté, il montre la misère et les } \\
\text { espoirs des migrants clandestins et la réaction des } \\
\text { villageois grecs, offrant un croisement de point de } \\
\text { vue. Le texte géniteur ne contient aucune annonce } \\
\text { d'un tel changement. }\end{array}$ \\
\hline $\begin{array}{c}\text { Hyperlien } 9 \\
\text { « dans le cadre de } \\
\frac{\text { l'opération Hermès. " }}{\begin{array}{c}\text { potentiel d'action } \\
\text { explicatif }\end{array}}\end{array}$ & $\begin{array}{l}\text { L'hyperlien extro-projectif sans indication des } \\
\text { sources mène vers le blog d'un journaliste, et } \\
\text { pourrait annoncer une relation dialogique. Le billet } \\
\text { se contente pourtant de décrire l'opération Hermès, } \\
\text { fournissant des explications complémentaires. }\end{array}$ \\
\hline $\begin{array}{l}\begin{array}{c}\text { Hyperlien } 10 \\
\text { «l'accueil et de } \\
\text { l'interrogatoire }\end{array} \\
\text { des migrants" potentiel } \\
\begin{array}{c}\text { d'action croisant } \\
\text { des points de vue }\end{array}\end{array}$ & $\begin{array}{l}\text { L'hyperlien extro-projectif sans indication des } \\
\text { sources mène encore vers France } 24 \text {. Alors qu'aucun } \\
\text { croisement de points de vue n'est annoncé dans le } \\
\text { texte géniteur, le texte relié, augmenté d'une vidéo, } \\
\text { avance une forte remise en question de l'efficacité de } \\
\text { Frontex dans l'opération Hermès en reliant plusieurs } \\
\text { points de vue : d'un côté, l'UE a répondu à l'appel } \\
\text { d'aide de l'Italie en mobilisant Frontex; d'un autre } \\
\text { côté, cette mobilisation pourrait envoyer un signe } \\
\text { ambigu vers le monde arabe en pleine révolution ; il } \\
\text { faudrait plutôt se poser la question de la provenance, } \\
\text { de l'identité et des raisons de ces flux, avance } \\
\text { l'auteur. La fin du texte relié anticipe sur la question } \\
\text { de la Libye traitée plus loin, sous forme de citation } \\
\text { non référencée dans le texte géniteur. }\end{array}$ \\
\hline $\begin{array}{l}\text { Hyperlien } 11 \\
\text { « Sylvie Guillaume } \\
\text { potentiel d'action } \\
\text { renvoyant à la source }\end{array}$ & $\begin{array}{l}\text { Répondant aux attentes potentielles d'un lecteur } \\
\text { voulant accéder aux sources des paroles citées, } \\
\text { l'hyperlien extro-projectif sans indication des sources } \\
\text { mène vers le bureau d'information du Parlement } \\
\text { européen, et plus précisément vers l'interview avec } \\
\text { l'eurodéputé : elle y avance un point de vue critique } \\
\text { envers Frontex. Certains arguments sont repris } \\
\text { sous forme de citation référencée dans le texte } \\
\text { géniteur. Le texte relié apporte des compléments à } \\
\text { l'argumentation principale de l'auteur. }\end{array}$ \\
\hline
\end{tabular}




\begin{tabular}{|c|c|}
\hline $\begin{array}{l}\text { Hyperlien } 12 \\
\text { « Eurodac » } \\
\text { potentiel d'action } \\
\text { définissant }\end{array}$ & $\begin{array}{l}\text { Répondant aux attentes potentielles d'un lecteur à } \\
\text { la recherche d'une définition de la base de données } \\
\text { Eurodac, l'hyperlien extro-projectif sans indication } \\
\text { des sources renvoie vers Wikipédia. Le nouveau } \\
\text { recours à Wikipédia constitue potentiellement une } \\
\text { trace des pratiques informationnelles du journaliste. }\end{array}$ \\
\hline $\begin{array}{l}\text { Hyperlien } 13 \\
\text { « comme Malte a du mal à } \\
\text { faire face " } \\
\text { potentiel d'action croisant } \\
\text { des points de vue }\end{array}$ & $\begin{array}{l}\text { L'hyperlien extro-projectif sans indication des } \\
\text { sources mène vers le site belge lalibre.be, et propose } \\
\text { un changement de perspective : d'un côté, sont } \\
\text { confirmés les problèmes auxquels des États comme } \\
\text { Malte sont confrontés suite aux réponses insuffisantes } \\
\text { de l'UE ; d'un autre côté, le sort tragique et touchant } \\
\text { d'un de ces migrants est exposé dans l'article. }\end{array}$ \\
\hline $\begin{array}{c}\text { Hyperlien } 14 \\
\text { « également menacé " } \\
\text { potentiel d'action croisant } \\
\text { des points de vue }\end{array}$ & $\begin{array}{l}\text { Menant encore vers le site de France 24, l'hyperlien } \\
\text { extro-projectif sans indication des sources fait } \\
\text { accéder à un point de vue divergent : d'un côté, il } \\
\text { énumère les raisons avancées par la Grèce pour la } \\
\text { construction d'une clôture; d'un autre côté, il avance } \\
\text { des arguments de l'UE concernant l'inefficacité de } \\
\text { cette mesure. L'avis de l'UE est intégré sous forme de } \\
\text { citation invisible dans le texte géniteur. Le texte relié, } \\
\text { augmenté d'une vidéo, anticipe aussi sur la position } \\
\text { de la Libye évoquée plus loin dans le texte géniteur. }\end{array}$ \\
\hline $\begin{array}{c}\text { Hyperlien } 15 \\
\text { «soutien de Paris " } \\
\text { potentiel d'action croisant } \\
\text { des points de vue }\end{array}$ & $\begin{array}{l}\text { L'hyperlien extro-projectif sans indication des } \\
\text { sources mène vers un article du Monde qui présente } \\
\text { plusieurs arguments et contre-arguments : d'un côté, } \\
\text { le texte relié cite les justifications du soutien de la } \\
\text { part du gouvernement français ; d'un autre côté, } \\
\text { il résume les réactions embarrassées de l'UE, et } \\
\text { situe le projet d'une clôture dans le contexte d'une } \\
\text { condamnation de la Grèce par la Cour européenne } \\
\text { des droits le l'homme. Sous forme de citation non } \\
\text { référencée, les arguments de l'UE sont repris dans } \\
\text { le texte géniteur. }\end{array}$ \\
\hline $\begin{array}{c}\text { Hyperlien } 16 \\
\text { «par la Cour européenne } \\
\frac{\text { des droits de l'homme }}{\text { potentiel d'action }} \\
\text { explicatif }\end{array}$ & $\begin{array}{l}\text { L'hyperlien extro-projectif sans indication des } \\
\text { sources mène vers la Tribune de Genève. Le texte } \\
\text { géniteur fournit des explications complémentaires } \\
\text { de la réaction de la Cour européenne des droits de } \\
\text { l'homme, et répond aux attentes informationnelles. } \\
\text { En mars 2012, ce texte n'est pourtant plus accessible; } \\
\text { le lecteur est redirigé vers la une de la Tribune de } \\
\text { Genève : un lieu d'indétermination imprévu par } \\
\text { l'Auteur se crée ainsi, entre texte géniteur et texte } \\
\text { relié. }\end{array}$ \\
\hline
\end{tabular}




\begin{tabular}{|c|c|}
\hline $\begin{array}{c}\text { Hyperlien } 17 \\
\text { « confier la surveillance } \\
\text { de nos frontières à la } \\
\text { Libye de Kadhafi » } \\
\begin{array}{c}\text { potentiel d'action } \\
\text { ironisant }\end{array}\end{array}$ & $\begin{array}{l}\text { L'hyperlien extro-projectif sans indication des } \\
\text { sources mène vers Romandie news. Le texte relié } \\
\text { fournit des explications complémentaires sur les } \\
\text { menaces de Tripoli quant à la gestion des flux de } \\
\text { migrants, confirmant ainsi le renversement ironique } \\
\text { anticipé par le texte géniteur. En mars } 2012 \text {, ce texte } \\
\text { relié n'est pourtant plus accessible ; le lecteur est } \\
\text { redirigé vers la une de la Tribune de Genève : un lieu } \\
\text { d'indétermination imprévu par l'auteur se crée entre } \\
\text { texte géniteur et texte relié. }\end{array}$ \\
\hline $\begin{array}{c}\text { Hyperlien } 18 \\
\text { « la solution face aux } \\
\text { crises migratoires » } \\
\text { potentiel d'action croisant } \\
\text { des points de vue }\end{array}$ & $\begin{array}{l}\text { L'hyperlien extro-projectif sans indication des } \\
\text { sources mène vers le site de TF1 news. Le texte } \\
\text { relié fournit des compléments d'information sur les } \\
\text { solutions proposées par le gouvernement français } \\
\text { face aux crises migratoires, tout en donnant accès à } \\
\text { d'autres opinions, notamment celui du Front national, } \\
\text { et la position d'autres pays européens. }\end{array}$ \\
\hline
\end{tabular}

\section{Synthèse de l'analyse}

Lors d'une dernière étape, j'ai élaboré une synthèse détaillée des répertoires du texte géniteur et des textes reliés, des figures de la lecture et des formes-modèles de la page-écran. Des propositions d’interprétation des lieux d’indétermination potentiels sont également avancées. Voici le résultat :

Suite aux flux migratoires venant de Tunisie, l’UE a décidé de mobiliser son agence Frontex. Selon le journaliste de marianne2.fr, l'Europe peine cependant à créer une véritable solidarité entre États. "L'immigration n'est pas qu'un problème national, elle peut concerner tout un continent, la crise de Lampedusa en est l'exemple ", avance le journaliste. L'hyperlien mène vers un autre article de marianne2.fr écrit par le même journaliste, et consacré à la crise de Lampedusa. Ce texte relié non seulement élargit l'argumentation du texte géniteur en abordant d'autres flux migratoires ; il déplace également le focus du texte géniteur en citant les réactions politiques face à cette crise. L'abolition des frontières en Europe a des conséquences sur les migrations. Au lieu de faire émerger une solution européenne commune, la crise de Lampedusa donne un exemple de « cette désunion européenne » que l'article fustige. L'hyperlien mène au même article convoqué auparavant.

Face à la crise migratoire, l'Europe a mis en place un outil : " l'agence Frontex », dont le lecteur peut consulter par hyperlien la définition sur Wikipédia. Les principales missions de l'agence sont reprises dans le texte 
géniteur. La lecture du texte relié sur Wikipédia ne fait que confirmer les informations avancées dans le texte géniteur : Frontex est chargé d'étudier les mouvements migratoires, de coordonner leur gestion entre États membres et d'organiser des vols de retour des migrants refoulés vers leurs pays d'origine.

"Elle a mené 43 opérations de contrôle ou de vols retours en 2010 selon des chiffres des eurodéputés Europe Écologie, un chiffre qui paraitt faible vu l'enjeu ", commente le journaliste. L'hyperlien donne accès au rapport des eurodéputés, qui va cependant beaucoup plus loin que la critique des chiffres : il accuse l'agence de violation des droits de l'homme. Ces accusations ne sont ni reprises ni commentées dans le texte géniteur ; elles sont néanmoins reliées à celui-ci par hyperlien, minant potentiellement l'interprétation du réel avancée par le texte géniteur.

"Avec les Rabits », Frontex possède un bras armé ; le journaliste attire l'attention du lecteur sur l'étrange nom de cette troupe. Le texte relié donne une définition neutre de l'équipe. Ses membres peuvent intervenir rapidement à la demande d'un État membre. Ils gardent leurs uniformes nationaux " mais ont un brassard de l'UE » : cette information est confirmée par une illustration montrant un Rabit avec son brassard.

"Ces équipes sont notamment intervenues en Grèce à l'automne dernier face à un afflux de migrants venant de la frontière avec la Turquie ", poursuit le journaliste. Alors que le premier hyperlien confirme l'information que les Rabits ont été envoyés en Grèce en donnant quelques compléments d'information sur le ton neutre d'une dépêche, l'hyperlien sur " un afflux de migrants » ouvre le texte géniteur vers d'autres points de vue : un reportage de France 24 montre d'un côté la nécessité de gérer les flux migratoires, et d'un autre côté attire l'attention sur le désespoir des réfugiés. Cette humanisation de la question des migrations est absente du texte géniteur, qui se concentre sur la critique d'un manque de coordination entre les pays européens.

Des experts ont été déployés par Frontex « dans le cadre de l'opération Hermès ", poursuit le journaliste. Le billet de blog relié au texte géniteur résume cette opération sur un ton neutre. Les experts sont " chargés de l'accueil et de l'interrogatoire des migrants ", affirme le journaliste de façon tout aussi neutre dans le texte géniteur, et cite un discours officiel selon lequel ces experts ne sont pas seulement policiers, mais « spécialistes des relations interculturelles ». Le texte relié, un article de France 24, met pourtant en question l'efficacité de Frontex dans cette opération en faisant dialoguer différents points de vue : d'un côté, l'UE a répondu à l'appel d'aide 
de l'Italie en envoyant ces experts ; d'un autre côté, cet envoi peut être interprété comme un signe ambigu envers le monde arabe. Il faudrait donc plutôt se concentrer sur la provenance et l'identité des flux migratoires.

Ces différentes perspectives ne sont ni reprises ni commentées dans le texte géniteur. Le journaliste se contente d'affirmer que l'UE paraît dépassée par les événements. "Ce qui est en cause, c'est que l'agence Frontex doit être assurée que les États membres fournissent les moyens promis », ajoute l'eurodéputé PS Sylvie Guillaume. Le contenu de l'interview reliée renforce les propos, déjà critiques, du texte géniteur envers l'Europe, renvoyant à la source d'information. Les réglementations pour l'asile posent également problème, poursuit le journaliste : le migrant doit faire sa demande dans le pays où il est arrivé en premier, et il peut être renvoyé dans ce pays. La base de données Eurodac, définie par un texte relié à Wikipédia, permet de repérer les demandeurs multiples.

Néanmoins, un petit État " comme Malte a du mal à faire face ». Le texte relié fournit des compléments d'information sur les problèmes à Malte. Il propose pourtant également un changement de perspective en présentant le sort tragique de l'un des migrants. Le journaliste de marianne2.fr renonce encore une fois à l'indication de la source dans le texte géniteur, sans pour autant annoncer le changement de perspective entre texte géniteur et texte relié. Il ne prend pas non plus position vis-àvis de celui-ci.

" La Grèce a également menacé » de construire une clôture à la frontière turque, poursuit le journaliste - le texte relié, un article de France 24, fait dialoguer plusieurs points de vue en avançant d'un côté les justifications de la Grèce, et en citant d'un autre côté les discours qui fustigent l'inefficacité de la solution. La Grèce a néanmoins obtenu « le soutien de Paris " - dans le texte relié, un article du monde.fr, le lecteur peut découvrir comment Brice Hortefeux justifie cette prise de position. Les réactions de l'UE, fortement embarrassée à cause de la condamnation de la Grèce par la Cour européenne des droits de l'homme, se trouvent juxtaposées aux justifications avancées par le gouvernement. L'argument de la condamnation de la Grèce est repris dans le texte géniteur. Les renvois de migrants vers la Grèce sont « contestés par la Cour européenne des droits de l'homme " parce qu'Athènes ne respecte pas assez les droits des demandeurs d'asile - le texte relié de la Tribune de Genève donne un fondement supplémentaire à cette critique.

Face à toutes ces difficultés, une solidarité entre les États fait décidément défaut - et le journaliste fait remarquer sur un ton potentiellement 
ironique : "Bien sûr, il reste la solution de confier la surveillance de nos frontières à la Libye de Kadhafi ". Le lien vers Romandie news résume les menaces de Tripoli concernant la " gestion » des flux de migrants passant par la Libye, et confirme l'ironie du propos. En France, François Fillon réaffirme que " la solution face aux crises migratoires » doit être européenne. Le texte relié juxtapose différentes propositions de solutions, dont celle du Front national, et revient sur la situation en Italie. L'auteur termine son article en constatant que la voie vers de véritables solutions sera encore longue.

Pour résumer, l'on constate que l'article donne à travers les textes reliés accès à une large documentation, invitant le lecteur à rentrer " par clic » dans l'atelier d'écriture du journaliste. Les textes reliés ne proposent pourtant pas seulement des définitions, des illustrations ou des compléments d'informations répondant à la présomption d'information du lecteur. En faisant dialoguer arguments et contre-arguments, et en déplaçant parfois la perspective du texte géniteur, ils esquissent une critique profonde envers l'agence Frontex - critique qui, dans le texte géniteur, se concentre uniquement sur le manque de coordination entre les États membres.

La critique la plus radicale envers Frontex est formulée dans le texte relié signé par les eurodéputés écologistes. En citant explicitement la source du texte relié dans le texte géniteur, le journaliste se désolidarise potentiellement de certaines accusations graves avancées par le rapport des eurodéputés. Cette distance semble plutôt abolie lorsqu'il s'agit, par texte relié, de prendre en compte les problèmes humanitaires posés par les migrations. Dans certains cas, les arguments avancés dans le texte relié se trouvent intégrés dans le texte géniteur. Toute indication de source manque alors, renforçant l'impression que le journaliste approuve les arguments du texte relié.

L'analyse de la pratique de l'hyperlien dans cet article permet de faire émerger l'attitude finement nuancée du journaliste envers ses sources ; les textes reliés semblent parfois utilisés pour ouvrir, complexifier, voire dialectiser et subvertir l'argument principal. Les hyperliens déplaçant le focus ou croisant des points de vue peuvent miner le texte géniteur. Est notamment intégré, par texte relié, le point de vue des migrants. Cette juxtaposition des points de vue préfigure un lecteur prêt à mettre en perspective des visions divergentes sur une même problématique. L'incertitude du lecteur pourrait trouver un exutoire dans l'espace " commentaires». Certaines formes-modèles pro-interventives de marianne2.fr encouragent ces pratiques. 


\section{Synthèse de la méthodologie et perspectives}

Cette analyse d'un article journalistique visait à faire émerger de façon palpable la méthodologie de la rhétorique du texte numérique. Ci-dessous, je résume encore une fois les principales étapes de cette méthodologie qui, en dehors du champ de la recherche sur le texte numérique, peut trouver sa place dans des démarches pédagogiques visant l'acquisition d'une culture informationnelle et d'une culture des médias réflexives et critiques.

Encadré 3. Méthodologie d'analyse d'un texte numérique avec hyperliens

1. Circonscription du texte à lire. Étape notamment importante dans le domaine du journalisme, où chaque texte relié mène par hyperliens à d'autres textes reliés.

2. Identification des formes-modèles de la page-écran (voir typologie présentée plus haut).

3. Identification des répertoires du « texte géniteur » (du texte qui contient les hyperliens), éventuellement paragraphe par paragraphe : circonscription des savoirs, compétences et normes auxquels le texte géniteur fait appel chez le lecteur.

4. Pour chaque hyperlien, relevé d'un catalogue d'attentes potentielles: identification des éventuelles annonces et reprises du texte relié dans le texte géniteur, et prise en compte des figurations les plus courantes de l'hyperlien (voir chapitre II).

5. Identification, pour chaque texte relié, des répertoires et stratégies.

6. Mise en perspective des attentes potentielles envers chaque hyperlien avec les répertoires et stratégies de chaque texte relié.

7. À partir du résultat de cette mise en perspective, identification des figures de la lecture de l'hyperlien.

8. Élaboration d'une synthèse détaillée de l'analyse. Éventuellement propositions d'interprétation des lieux d'indétermination.

Cette analyse pourrait se poursuivre par l'analyse des commentaires laissés par les lecteurs.

Dans les analyses du corpus journalistique, j’ai laissé de côté le potentiel d'action temporel de certains hyperliens. En effet, la plupart des hyperliens renvoient vers des textes reliés qui ont été rédigés avant la date de publication du texte géniteur, relatant des événements d'un passé plus ou moins proche. Certaines hyperfictions vont plus loin dans l'exploration de ce potentiel d'action temporel. À travers l'analyse de l'une d'entre elles, je montrerai maintenant que certaines figures rappellent celles $d u$ discours informatif et argumentatif, alors que d'autres sont spécifiques au discours narratif. 


\section{FIGURES DE LA LECTURE ET FORMES-MODÈLES DE LA PAGE-ÉCRAN DANS LE DISCOURS NARRATIF}

\section{Brève introduction à la littérature numérique et justification du choix de l'exemple}

Il s'agit à présent d'identifier les figures de la lecture du texte numérique mobilisées dans le discours narratif. Le choix d'une hyperfiction me paraît propice à cette entreprise, grâce au champ des possibles particulièrement large expérimentés par certains auteurs. Au lieu de constituer un corpus de plusieurs hyperfictions et d'en retenir seulement certaines parties pour l'analyse, j'ai décidé de me concentrer ici sur un seul exemple, et de l'analyser en détail. Je suivrai pour cette analyse la méthodologie exposée dans la partie « Synthèse de la méthodologie et perspectives », p. 182.

Les premières hyperfictions aux États-Unis [comme Afternoon a story de Michael Joyce, 1993] s’inspiraient souvent du roman expérimental papier. $\mathrm{Au} \mathrm{xx}$ e siècle, il paraissait décidément impossible pour certains auteurs d'organiser encore les événements sous forme de totalité intelligible. Les structures temporelles et causales du roman « traditionnel » ne semblaient plus pouvoir contenir la représentation d'un réel qui, dans la perception de ces auteurs (Robert Musil, Alain Robbe-Grillet, Claude Simon, James Joyce, parmi tant d'autres) se déployait dans un tissu complexe de causalités entrelacées.

La charpente logicotemporelle de la narration de tradition aristotélicienne était non seulement perçue comme inapte à rendre compte de la complexité du monde ; elle était dénoncée comme reflet d'une société sclérosée. Comme je l'ai déjà brièvement indiqué au chapitre II, des auteurs tel Alain Robbe-Grillet ont donc essayé de tisser des réseaux d'actions et de motifs au lieu de raconter des histoires suivant le schéma classique de l' « avant » et " après ». Pour Claude Simon, le récit devait désormais se construire en référence au texte et à la logique de la langue travaillée. Parallèlement s'est développée une nouvelle conscience du support de lecture.

Plusieurs auteurs ont rapidement considéré l'hypertexte comme un procédé particulièrement adapté pour relier les éléments d'un tissu « non séquentiel » de textes de façon " rhizomatique » [la référence aux Mille plateaux de Gilles Deleuze et Félix Guattari a fréquemment été convoquée par ces auteurs, voir Landow, 1997, 2, 38 ; Bolter, 1991, 143]. La figuration 
non séquentielle de l'hypertexte s'est trouvée opposée à la séquentialité $\mathrm{du}$ livre papier. Les auteurs la mobilisaient notamment pour justifier leur refus de la clôture du texte et des enchaînements chronologiques et causaux. L'hypertexte a été associé à une logique de fractionnement.

La dissonance cognitive potentiellement provoquée par l'hypertexte s'est trouvée valorisée, mise au profit d'une désorientation tout à fait assumée. Beaucoup de chercheurs et d'auteurs de créations hypermédiatiques considéraient ainsi dans les années 1990-2000 la lecture " immersive » d'un texte narratif comme fondamentalement incompatible avec les caractéristiques du dispositif numérique [Landow, 1997 ; Bolter, 1991 ; Joyce, 1993]. Marie-Laure Ryan qualifie la position du lecteur dans une hyperfiction d'« externe » et " observante » $[2006,107]$.

Plus rares étaient ceux qui, dès ces années, insistaient sur le potentiel d'action temporel et causal de l'hyperlien dans la narration [Norton, Zimmerman, Lindeman, 1999 ; Bouchardon, 2002 ; Saemmer, 2003, 2007]. Se détachant des paradigmes du roman expérimental des années 1950 à 1970, des auteurs comme Renée Bourassa [2010] revalorisent aujourd'hui les bienfaits de la contiguité logicotemporelle et de l'immersion.

Dans le roman contemporain se dessine depuis plusieurs années une tendance vers ce que Bruno Blanckeman [2000] a appelé la « re-narrativisation » du texte (voir aussi chapitre II). Pour les auteurs s'inscrivant dans cette tendance, il s'agit, comme le formule Blanckeman, de montrer comment une fiction s'élabore désormais sur sa propre ruine - ruine laissée par les expérimentations des avant-gardes de la seconde moitié du $\mathrm{xx}^{\mathrm{e}}$ siècle. Les fictions re-narrativisantes explorent les contiguités possibles entre événements racontés et éléments décrits tout en faisant émerger les fissures inexorables de cette interprétation du réel. Se préfigurent dans ces récits des pratiques de lecture oscillant entre une jouissance de l'effet de présence et une distance réflexive. Pour le domaine de l'hyperfiction numérique, Marie-Laure Ryan a qualifié cette lecture de "medium-aware immersion" [2001, 351]. Elle est notamment préfigurée par des procédés rhétoriques comme le kiné-trope présenté au chapitre précédent.

Les procédés rhétoriques et les formes-modèles de cette immersion " consciente du dispositif » sont explorés de façon particulièrement diversifiée dans l'hyperfiction allemande Zeit für die Bombe de Susanne Berkenheger $^{8}$ que j'analyserai donc en guise d'exemple représentatif. Après un relevé des procédés rhétoriques et des formes-modèles je

8. Susanne Berkenheger, Zeit für die Bombe, [En ligne] : < http://www.wargla.de/zeit.htm >. 
proposerai, comme je l'ai fait pour l'article journalistique « 22 », une interprétation possible de cette œuvre et de ses lieux d'indétermination.

Avant d'entreprendre l'analyse, je reviens brièvement à l'histoire de la littérature numérique dans laquelle s’inscrit l'exemple choisi. Les premières expérimentations littéraires sur support numérique remontent à 1959, aux créations de Theo Lutz en Allemagne et à celles de Brion Gysin aux États-Unis. Que l'on pense aux générateurs de textes s'inscrivant dans l'héritage de l'OuLiPo (Ouvroir de Littérature Potentielle explorant la génération textuelle sur papier à partir des années 1960), à la poésie animée et à l'hyperfiction (expérimentée depuis les années 1980), ou aux blogs littéraires et aux récits sur Twitter et Facebook qui ont émergé plus récemment avec le développement du Web 2.0, la littérature numérique n'a cessé d'évoluer ces dernières décennies.

Bien qu'il soit souvent difficile de tracer des frontières nettes entre littératures et arts numériques, des bases de données volumineuses aux États-Unis ${ }^{9}$, au Canada ${ }^{10}$ et en Europe ${ }^{11}$ recensent aujourd'hui les œuvres considérées comme appartenant au champ, proposent des classements par genre et engagent des démarches de légitimation passant en outre par l'établissement d'anthologies [voir Saemmer, 2011c]. La recherche et la création s'organisent autour d'événements internationaux, qui s'efforcent de réunir différentes communautés de créateurs et chercheurs ${ }^{12}$.

Malgré les difficultés pour circonscrire le champ avec exactitude, je propose de retenir ici quelques caractéristiques fondamentales de la littérature numérique. Il y a d'abord la présence nécessaire d'une forme de texte sur la surface observable du support de lecture. En effet, et même si cette affirmation peut prêter à discussion, j'avancerais que la seule présence d'éléments textuels dans le programme d'une œuvre numérique n'est pas suffisante : sinon la plupart des créations cinématographiques actuelles, les jeux vidéo, et même n'importe quelle photographie numérique feraient partie intégrante du champ de la littérature numérique définition qui me paraît bien trop large pour être opérationnelle.

Le caractère programmé de l'œuvre est néanmoins une caractéristique importante de la littérature numérique, qui permet de la démarquer de ses

9. Directoire de la Electronic Literature Organization, [En ligne] : < http://directory.eliterature. org/ >.

10. Répertoire des arts et littératures hypermédiatiques, [En ligne] : < http://www.labo-nt2.uqam.ca/ observatoire/repertoire $>$.

11. Base de données du projet européen ELMCIP, [En ligne] : < http://elmcip.net/knowledgebase >.

12. Par exemple, le cycle de conférences et le festival e-poetry, et les conférences internationales de la Electronic Literature Organization. 
prédécesseurs : la poésie vidéo ou la littérature combinatoire sur papier. Le programme contient des " représentations " (voir réflexions sur la balise nofollow plus haut, les recherches de Nick Montfort et Stephanie Strickland [2012] sur les commentaires contenus dans certains programmes, les travaux de Mark Marino [2006] sur les figurations culturelles dans le code, et la démarche d'analyse croisée des événements de surface et du programme entreprise par Philippe Bootz et moi-même [2012]). Le programme constitue aussi la seule partie résistant à la " labilité du dispositif » provoquée par le changement de systèmes, de logiciels et de vitesse de calcul des ordinateurs, qui fragilise les événements de surface sur l'écran. Les auteurs de littérature numérique ne peuvent guère ignorer cette labilité, et se positionnent de diverses façons par rapport à elle, essayant de la maîtriser ou au contraire de la laisser agir de manière incontrôlée, adhérant pour certains à une esthétique que j'ai qualifiée d'« éphémère » [en allusion à l'essai du même titre par Christine Buci-Glucksmann, 2003].

Les événements observables à la surface de l'œuvre sont parfois caractérisés par des couplages entre texte et mouvements. J'ai avancé plus haut l'hypothèse que certains énoncés de mouvements constituent des icônes, c'est-à-dire des signes qui s'approchent par leur structure visuellement percevable d'une représentation de leur référent d'expérience. J'ai proposé d'appeler ces icônes unités sémiotiques temporelles à cause de leur lien étroit avec les unités du même nom dans le domaine sonore. L'unité « obsessionnel » par exemple est, dans le sonore comme dans le visuel, caractérisée par une pulsation rapide, qui peut rappeler des éléments clignotants comme des boutons d'alarme.

Le potentiel d'action de ces unités ne se démarque guère de leur référent d'expérience : l'obsessionnel peut ainsi renvoyer à des idées d'urgence et de danger. En couplant une unité sémiotique temporelle à un texte, l'auteur d'une création numérique effectue un acte d'interprétation de leur relation, et anticipe sur les réactions possibles du lecteur. L'irradiation iconique peut être poussée à l'extrême de sorte que le mot se trouve au moins partiellement transformé en simulacre de référent (par exemple, le mot "* *œur* » qui pulsionne dans le récit d'une histoire d'amour). Un tel procédé préfigure un lecteur qui accepte de s'immerger dans la matérialité des éléments animés et de jouir de leurs effets de présence, mettant au moins transitoirement de côté son incrédulité vis-à-vis des pouvoirs de représentation du texte.

D'autres couplages rhétoriques entre texte et unités sémiotiques temporelles, en revanche, préfigurent plutôt une mise au défi des attentes du 
lecteur, proposant des relations surprenantes, insolites ou provocatrices entre le potentiel d'action du texte et du mouvement. Je considère ces lieux d'indétermination comme un facteur important de littérarité [voir Saemmer 2007, 2008, 2009, 2001a], mais ce n'est pas le lieu ici de discuter la question de la littérarité de l'œuvre numérique. M'importe avant tout le constat que l'animation textuelle est un trait caractéristique de certaines formes de littérature numérique.

Une autre propriété concerne sa dimension interactive. En effet, le texte devient manipulable de diverses manières : le lecteur peut cliquer, déplacer des lettres ou mots, provoquer des réactions sur l'interface en effleurant ou en grattant des éléments, voire en insérant lui-même des contenus. Certains énoncés de gestes constituent le signifiant d'un signe iconique que j'ai proposé d'appeler unité sémiotique de la manipulation. Comme pour les unités sémiotiques temporelles, la reconnaissance d'une unité sémiotique de la manipulation est fondée sur des processus d'intégration et de stabilisation d'expériences antérieures. Une unité comme " activer », présente dans tout hyperlien, mobilise un potentiel d'action qui ne se démarque guère de son référent d'expérience : rappelant des gestes d'appui bref et non répétitif que nous effectuons par exemple sur des interrupteurs, elle renvoie potentiellement à des idées de réactivité, de maîtrise et de déclenchement instantanés. L'actualisation de ce potentiel d'action s'effectue en fonction du texte auquel il est couplé, ainsi qu'en fonction de l'horizon d'attente du lecteur.

Lorsque l'irradiation iconique mobilisée par une unité sémiotique de la manipulation est poussée à l'extrême, il peut se créer dans le couplage entre texte et gestes un simulacre de référent qui constitue un puissant facteur d'immersion. Autrement dit, le lecteur peut avoir l'impression de manipuler non plus tellement un mot, mais la chose ou le concept évoqués. Ces couplages anticipent sur un lecteur prêt à s'immerger dans les environnements ou événements décrits ou racontés, ce qui nécessite encore une fois la mise entre parenthèses, au moins transitoire, de son incrédulité par rapport aux pouvoirs de représentation du texte (voir exemples de kiné-grammes dans le corpus journalistique).

Il arrive cependant, là aussi, que des lieux d'indétermination s'ouvrent entre le potentiel d'action du texte et de l'unité sémiotique de la manipulation, qui mettent au défi les attentes du lecteur (par exemple, lorsqu'il est invité à gratter le mot « eau » dans un poème numérique). J'ai proposé le terme kiné-trope pour caractériser ces couplages qui pourraient encore être considérés comme un important facteur de littérarité, 
même si l'analyse d'exemples journalistiques a montré que l'emploi de ces figures n'est pas qu'une question de genre. Je retiens ici surtout le constat que la littérature numérique expérimente fréquemment avec le texte manipulable.

Tout texte manipulable relie un état avant et un état après l'effectuation des gestes. J'ai montré plus haut, à partir de l'étude du corpus journalistique, comment le texte relié par hyperlien à un texte géniteur peut confirmer les attentes du lecteur. J'ai également relevé quels procédés rhétoriques de l'hyperlien peuvent plutôt mettre ces attentes au défi. Cette mise au défi est également expérimentée dans la littérature numérique.

\section{Traces de réception}

J'en viens à l'exemple sélectionné pour ce chapitre consacré au discours narratif. L'hyperfiction Zeit für die Bombe, un polar parsemé d'hyperliens, a suscité beaucoup de commentaires et d'analyses depuis sa date de publication en 1997. Le relevé de ces « traces de réception » me permettra de comparer l'horizon d'attente au moment de la publication à des évolutions plus récentes de celui-ci - approche diachronique considérée comme essentielle par les fondateurs de la théorie de la réception tel Hans Robert Jauss [1972-1978 ; 2010], et qu'il m'est possible de mettre ici en œuvre grâce aux nombreux travaux consacrés à cette création.

Certains premiers lecteurs de Zeit für die Bombe ayant laissé des traces de leur expérience (il s'agit souvent de lecteurs académiques) ont eu recours à la figuration du "wreader" proposée par George P. Landow [1997], et à la représentation de l'hyperfiction comme contre-modèle au récit classique : « la non-linéarité permet de créer toujours un autre cours de l'histoire ", " le lecteur influence le cours de l'histoire de façon décisive » [Hautzinger, 1999 ; c'est moi qui traduis]. Ce sont souvent la perte de repères et la désorientation qui se trouvent mises en avant dans les traces d'expériences de la fin des années 1990. Ces impressions peuvent, d'une part, être mises en relation avec l'horizon d'attente des lecteurs académiques de l'époque, potentiellement imprégné par les figurations américaines de l'hypertexte ; d'autre part, elles reflètent sans doute le ressenti de lecteurs qui découvrent le genre de l'hyperfiction, mais se sentent encore dépourvus de repères pour la lire.

Il faut rappeler qu'à la fin des années 1990, en dehors des figurations littéraires de l'hyperlien réservées souvent aux milieux académiques, l'imaginaire d'un hyperlien informationnel ayant comme objectif de diminuer 
les incertitudes du lecteur s'était déjà bien imposé (voir chapitre II). La vision de l'hyperlien comme lien " efficace » et " prédictible » entre des informations factuelles a été présentée comme un "standard » répondant aux attentes du lecteur [Charney, 1994]. Dans ce contexte où l'hyperfiction s'établissait donc à la fois comme contre-modèle à la narration traditionnelle associée au papier, et comme contre-modèle aux paradigmes du site Web informationnel, Roberto Simanowski écrit en 1999 que le lecteur ne sait jamais comment les éléments de Zeit für die Bombe sont connectés, quelle est la relation entre les personnages et " ce que tout cela signifie » (c'est moi qui traduis). Mathias Franke avance de même que le lecteur peut choisir entre plusieurs « variantes » de l'histoire, mais que le récit ne lui donne aucune indication permettant de faire ces choix en connaissance de cause. Les interactions relèveraient avant tout du hasard ; Zeit für die Bombe serait une « œuvre chaotique " (c'est moi qui traduis).

Face à cette sensation de lecture chaotique, parfois accueillie positivement comme un défi [Simanowski, 1999] et parfois fustigée [Franke], certains lecteurs-chercheurs, peut-être influencés par la profusion des graphes de sites dans les années 1990, ont décidé de dessiner la " carte de navigation » de Zeit für die Bombe [Runkehl, Schlobinski, Siever, 1998, 166-167], sans pour autant se pencher sur les relations de contiguité temporelle ou causale qui s'établissent.

L'analyse par Beat Suter publiée en 2005 se démarque fortement de ces premiers écrits, car elle met en avant les contiguïtés possibles entre les textes de Zeit für die Bombe. Le retour sur certaines pages déjà explorées lors d'un premier parcours permet de regrouper les différents passages autour d'événements tout en les montrant parfois sous une nouvelle perspective, affirme Suter. L'expérience de lecture rappellerait la démarche d'un détective, et déboucherait sur la reconstruction d'une « histoire » cohérente.

Ce n'est pas seulement l'exploration des hyperliens en tant qu'éléments associatifs ou dissociatifs qui a retenu l'attention des lecteurs. Dans les années 2000 à 2010, certains reprennent la lecture de Zeit für die Bombe et proposent une analyse plus détaillée, insistant moins sur la perte de repères que sur la possibilité et/ou la mise au défi de la lecture immersive. Roberto Simanowski avance en 2007, dans un texte intitulé de façon significative "What is and to What End Do We Read Digital Literature?", que les gestes de manipulation, mobilisés à certains moments dans Zeit für die Bombe, peuvent donner l'impression au lecteur qu'il porte une responsabilité dans le déroulement des événements racontés. En même temps, Simanowski souligne que le texte réfléchit dans ces passages son propre 
dispositif, sans préciser les modalités de cette réflexion. Les animations $\mathrm{du}$ texte, qui mettent principalement en œuvre des clignotements rapides, sont soit analysées comme des effets de " présentification » qui attirent l'attention du lecteur sur « le temps qui passe » [Hautzinger, 1999, c'est moi qui traduis], soit considérées comme des redondances [Simanowski, 1999].

La divergence entre les expériences de lecture exposées, qui thématisent soit une impression de décohérence complète, soit la possibilité d'une contiguité temporelle et logique entre les parties de l'hyperfiction, et qui verbalisent une implication du corps du lecteur mi-immersive, miréflexive sans aller plus en avant dans l'analyse de ces effets, m'a incitée à examiner avec précision les figures de la lecture et des formes-modèles de Zeit für die Bombe.

\section{Formes-modèles de la page-écran et du texte}

L'hyperfiction Zeit für die Bombe a été écrite et programmée par Susanne Berkenheger en 1997, bien avant le succès des systèmes de gestion de contenu et leur influence sur la conception et la lecture de sites Web. Une lecture exhaustive des textes, disposés sur 47 pages fixes et 46 pages défilant automatiquement, est rythmée par l'activation de 76 hyperliens et dure environ quatre heures.

J'avance l'hypothèse que la présentation graphique des textes dans Zeit für die Bombe, leur cadrage sur la page-écran et la présence de pages défilantes peuvent mettre au défi les expectatives d'un lecteur en 2013 à plusieurs égards. La structuration proposée par les systèmes de gestion de contenu, aujourd'hui omniprésents sur le Web, a modifié les attentes du lecteur face au texte numérique : l'insertion d'un sommaire-menu en haut de chaque page-écran d'un site, la taille réduite du cadre accueillant le " texte courant ", le noir sur blanc et les polices de caractères sans empattements se sont généralisés ces dernières années, préfigurant un lecteur qui souhaite disposer d'une vue d'ensemble sur les textes disponibles et aller rapidement à l'information. Les textes de Zeit für die Bombe sont, au contraire, disposés sur des fonds de pages de couleurs diverses, s'affichent dans une police de caractère avec empattements en plusieurs couleurs et tailles qui contrastent de façon plus ou moins nette avec les couleurs du fond, et sont donc plus ou moins facilement déchiffrables. Ils s'étalent sur toute la longueur de la fenêtre de lecture. Aucun menu ne 
permet une vue d'ensemble sur le nombre de textes, les événements, les personnages et lieux.

La première page de Zeit für die Bombe sur laquelle s'affichent, sur fond noir encerclé de deux banderoles rouges, le titre et un signe graphique affecté d'un hyperlien (>>>), est représentative de la stratégie narrative qui préfigure un lecteur acceptant de plonger dans l'histoire racontée sans disposer de repères. Elle ne contient en effet aucune indication sur les contenus explorables. Lorsque le lecteur active l'hyperlien présent sur cette page-écran, plusieurs pages s'enchaînent automatiquement, sans que le lecteur ait à intervenir manuellement. De brefs morceaux de textes sont disposés sur ces pages ; apparaissant les uns derrière les autres, ils esquissent le point de départ d'une histoire : une jeune fille amoureuse, nommée Veronika, arrive à Moscou. Elle transporte une bombe dans sa valise. La couleur beige du fond de ces pages défilantes peut éventuellement rappeler celles d'un livre papier. La plupart de ces pages, que je propose d'appeler « transitionnelles » parce qu'elles assurent effectivement de façon automatisée la transition entre deux épisodes, s'affichent en beige ; un texte souvent formaté en noir s'y superpose.

Alors que la forme-modèle graphique de ces pages est donc potentiellement pro-intensive et encourage, par la police avec empattements et le rappel de la couleur papier, la lecture lente et intensive, le défilement automatique induit une certaine perte de maîtrise. En effet, le lecteur ne peut ni régler le rythme de défilement des pages transitionnelles, ni l'interrompre - à moins de quitter le site. Je propose d'appeler « prodepréhensive » une forme-modèle où le lecteur perd prise sur l'interface.

Mettant au défi les attentes de maîtrise et d'efficacité immédiate potentiellement associées à la manipulabilité du texte numérique, d'autres déclinaisons de cette forme-modèle sont mises en œuvre dans Déprise par Serge Bouchardon ${ }^{13}$ et dans ma création Tramway ${ }^{14}$. Lors du dernier épisode de Déprise, le lecteur est invité à écrire un texte dans une zone de saisie. Peu importent pourtant les lettres qu'il tape sur le clavier, c'est toujours le même texte qui apparaît dans cette zone. Dans Tramway, plusieurs fenêtres pop-up peuplent l'écran. Lorsque le lecteur essaie de les fermer, d'autres apparaissent toujours et remplissent progressivement la fenêtre de lecture. Les deux créations préfigurent un lecteur prêt à accepter la perte de prise sur le dispositif.

13. Serge Bouchardon, Déprise, [En ligne] : < http://lossofgrasp.com/ >

14. Alexandra Saemmer, Tramway, [En ligne] : < http://revuebleuorange.org/bleuorange/02/saemmer/ >. 
Les formes-modèles pro-depréhensives sont plus fréquentes en littérature numérique (voir aussi les « poèmes à lecture inconfortable » par Philippe Bootz) ${ }^{15}$ que dans les médias accueillant des discours informatif ou argumentatif. Il arrive néanmoins qu'elles soient mises en œuvre sur des sites Web commerciaux : les sites pornographiques par exemple, où les fenêtres pop-up résistent également avec obstination aux gestes de manipulation du lecteur.

Je reviens au début de Zeit für die Bombe. Après quelques pages-écran qui s'enchaînent automatiquement, une page fixe apparaît, contenant de nouveau le symbole graphique « >>> » affecté d'un hyperlien. Le texte s'affiche maintenant en blanc sur un fond gris, créant potentiellement une ambiance brumeuse, nuageuse, froide ; l'interprétation plus précise de cette couleur dépend des référents culturels du lecteur [voir Heller, 2009] et de ses expériences personnelles, et s'actualise éventuellement en relation avec le potentiel d'action du texte. Lorsqu'ils apparaissent en couleur rouge sur fond noir, certains mots ou phrases peuvent renvoyer à la violence, au crime et au danger avant même que le lecteur ne les déchiffre : un fond noir et des lettres rouges caractérisent ainsi la collection « Actes noirs » de l'éditeur Actes Sud qui a publié en outre la trilogie Millenium.

En effet, la couleur en tant que forme-modèle de la page-écran et du texte peut être perçue sur un mode iconique, même si certains artistes se sont insurgés contre l'idée d'établir des correspondances immédiates entre couleur et référent. Fernand Léger [1965, 111] affirme par exemple : « La couleur est vraie, réaliste, émotionnelle en elle-même sans se trouver dans l'obligation d'être étroitement liée à un ciel, à un arbre, à une fleur, elle vaut en soi ». C'est pourquoi, dans le Traité du signe visuel [1992], le groupe $\mu$ propose de différencier entre le " signe iconique » et le " signe plastique ». Une tâche circulaire et rouge peut être perçue soit sur le mode iconique (comme un ballon ou un coucher de soleil), soit sur le mode plastique (évoquant l'idée de circularité et de chaleur). L'orientation vers le signe iconique se définit ainsi par une prédominance de la fonction référentielle : voilà pourquoi j’ai proposé de parler de formes-modèles pro-référentielles. Dans le signe plastique, l'accent est mis sur le message pour son propre compte : je propose alors de parler de formes-modèles " non référentielles ».

Ce n'est pas seulement la couleur, mais aussi la forme engendrée par la disposition du texte, par exemple l'espace laissé vacant entre la

15. Philippe Bootz, Petits poèmes à lecture inconfortable, [En ligne] : < http://epoetry.paragraphe. info/artists/oeuvres/bootz/bootz_index.htm >. 
première et la deuxième phrase affichées en couleurs vives sur la pageécran reproduite ci-contre (figure 14), qui renvoient potentiellement au référent d'expérience d'un tâtonnement dans un lieu sombre. Le regard du lecteur est capté par cet espace avant même qu'il entame la lecture des textes affichés en couleurs vives ; sa main est invitée à saisir l'hyperlien logé dans cet espace sombre. L'agrandissement progressif de la taille des lettres entre les quatre paragraphes sur la page-écran pourrait préfigurer l'approche progressive d'un être, d'une chose ou d'un son dans cet espace sombre, et constitue donc également une forme-modèle pro-référentielle.

Le potentiel d'action de la couleur dans Zeit für die Bombe n'est pourtant pas systématiquement d'un ordre référentiel. Dans le cas de la pageécran citée, il serait difficile d'associer la couleur jaune de la deuxième phrase et la couleur verte de la première phrase à des référents d'expérience différents, bien que les couleurs ne soient pas les mêmes. Émerge donc dans Zeit für die Bombe une oscillation potentielle entre des formesmodèles que le lecteur aurait tendance à percevoir sur un mode iconique, et des formes-modèles non référentielles dont le sens ne se laisse pas enfermer dans le signe iconique [pour la différenciation entre signe iconique et signe plastique, voir aussi Roque, 2010].

Figure 14. Capture d'une page-écran de Zeit für die Bombe de Susanne Berkenheger

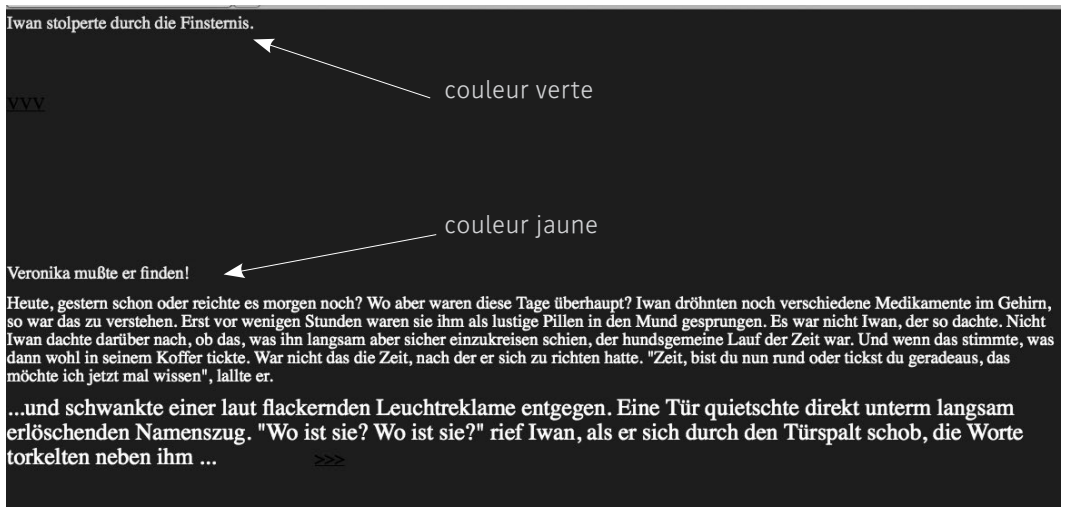

Certes, le lecteur peut traverser cette hyperfiction très rapidement, en activant la forme-modèle pro-référentielle qui marque les hyperliens en couleur rouge, et qui pourrait être associée aux traces de sang d'un crime ; il pourrait se contenter d'interpréter les ambiances potentiellement créées 
par les couleurs, la dimension des lettres, le défilement automatique de certaines pages et le clignotement de certains mots, sans entrer dans une lecture détaillée des textes. La longueur des textes ainsi que l'absence d’images ne préfigurent pourtant guère ces pratiques extensives.

Les hyperliens de Zeit für die Bombe changent systématiquement de couleur après avoir été activés. Il arrive ainsi que le lecteur retombe, au bout de l'exploration de plusieurs autres pages, sur une page-écran où il avait déjà activé un hyperlien. Le fait que cet hyperlien s'affiche maintenant dans une couleur différente lui fournit un repère potentiel pour poursuivre l'exploration selon une autre piste. Cette forme-modèle peut donc agir comme un marqueur dans un livre papier, et donner au lecteur l'impression rassurante qu'il aura, à un moment donné, fait le tour de toutes les pages.

Le tableau ci-dessous liste encore une fois les formes-modèles repérées dans Zeit für die Bombe.

\section{Tableau 12. Formes-modèles de la page-écran du texte}

Formes-modèles prodepréhensives

préfigurent un lecteur acceptant de perdre prise sur l'interface.

Formes-modèles proréférentielles préfigurent un lecteur prêt à s'abandonner, au moins transitoirement, au simulacre de référent proposé.

Formes-modèles non référentielles préfigurent un lecteur prêt à considérer les formes et couleurs comme des entités abstraites.
Pages-écran défilant automatiquement sans que le lecteur puisse intervenir sur le rythme, ou arrêter le processus ; multiplication intempestive de fenêtres pop-up sur la page-écran ; absence de réactivité de certains éléments d'apparence manipulables ; ou réaction différente de celle annoncée par le texte géniteur ou les formes-modèles.

La couleur, la police de caractère, la forme ou l'étendue de certains espaces sur la page-écran, les unités sémiotiques de la manipulation ou les unités sémiotiques temporelles renvoient potentiellement à un référent d'expérience.

La couleur, la police de caractère, la forme et l'étendue de certains espaces, des gestes et mouvements ne se laissent pas enfermer dans un signe iconique, mais renvoient plutôt à leur propre plasticité. 
Formes-modèles pro-intensives préfigurent des pratiques plutôt concentrées et lentes.

Formes-modèles pro-extensives préfigurent un lecteur impatient, souhaitant aller vite dans l'exploration du texte.
Police de caractère avec empattements ; relative longueur des textes, des paragraphes ; structuration des textes rappelant parfois un pendant papier.

Structuration des textes mettant en évidence les informations clés du texte ; encadrés synthétiques hyperliés ; police sans empattements ; haut niveau de tabularité de la page-écran ; marquage des hyperliens incitant à la navigation.

\section{Figures de la lecture de l'hyperlien et de l'animation textuelle}

Après l'examen des principales formes-modèles dans Zeit für die Bombe, je viens aux procédés rhétoriques de l'hyperlien et de l'animation textuelle. Contrairement aux articles du corpus journalistique pour lesquels il fallait d'abord définir le périmètre du texte à lire, Zeit für die Bombe forme une entité close dont le lecteur peut «faire le tour".

Pour chacune des pages-écran, j'ai d'abord identifié les répertoires du texte géniteur. J'ai circonscrit les attentes potentiellement suscitées par chaque hyperlien en fonction de ces répertoires, prenant en compte l'horizon d'attente possible du lecteur face à un texte narratif en général, et face à une hyperfiction en particulier (voir chapitre II). J'ai établi des tableaux comme dans l'analyse de l'article journalistique exposé plus haut - tableaux qui sont trop volumineux pour être reproduits ici. En listant les attentes, je suis partie de l'hypothèse que le lecteur accepte l'idée d'explorer un texte narratif sur un dispositif numérique, et qu'il est prêt à effectuer des lectures intensives, voire immersives des textes proposés - hypothèse qui, en regard des attentes fréquemment informationnelles du lecteur face au texte numérique et de la prédominance des pratiques extensives, ne va pas de soi.

Lors de l'étape suivante, j'ai mis ces attentes en perspective avec les répertoires de chaque texte relié afin d'évaluer le niveau de contiguité possible. Dans le discours narratif, cette contiguité peut se fonder en outre sur des relations temporelles et/ou causales, sur des changements de focus et sur le couplage entre texte, unité sémiotique de la manipulation et/ou unité sémiotique temporelle.

Lors d'une première lecture, le niveau de contiguïté entre certains textes géniteurs et textes reliés dans Zeit für die Bombe peut paraître 
faible, car marqué par des sauts temporels et/ou logiques importants (le personnage principal Veronika se trouve dans un taxi puis, dans le texte relié, est déjà en train de faire l'amour avec son amant Vladimir ; le texte géniteur renvoie à Veronika fuyant son ex-ami Iwan qui l'attendait à la gare de Moscou, alors que ce même Iwan se trouve dans le texte relié à l'hôpital psychiatrique). Si le lecteur explore tous les hyperliens, il peut cependant établir une contiguité logicotemporelle entre la plupart des épisodes. Ceux-ci s'ordonnent en effet progressivement sur l'axe temporel de l'histoire racontée ; les relations entre les personnages se clarifient, la description des lieux s'étoffe.

Voici une description plus précise des figures de la lecture que j'ai pu identifier dans Zeit für die Bombe.

\section{Figures de la lecture « chronologique »}

Le potentiel d'action de beaucoup de relations entre texte géniteur et texte relié dans Zeit für die Bombe est d'un ordre temporel. De tels procédés rhétoriques de l'hyperlien préfigurent un lecteur habitué à la charpente d'un texte narratif avec ses suites chronologiques, ses sauts temporels en avant et en arrière, et des événements présentés comme synchroniques. Dans un texte narratif publié sur papier, ces suites chronologiques ou sauts temporels peuvent advenir d'un chapitre à l'autre, d'un paragraphe à l'autre, voire parfois à l'intérieur d'une même phrase. Dans le cas de la lecture d'un texte numérique avec hyperliens, le lecteur ne peut pas évaluer l'étendue du texte à venir avant d'avoir activé le mot ou la phrase manipulables. En outre, l'hyperlien est parfois inséré au milieu d'un paragraphe. Le texte géniteur poursuit dans ce cas le récit d'un événement ou la description d'un lieu, alors que l'hyperlien propose au lecteur d'explorer un saut temporel en avant, un saut temporel en arrière ou un événement qui se déroule parallèlement dans un autre lieu. Les choix à faire par le lecteur, entre la poursuite de la lecture du texte géniteur ou l'activation d'un hyperlien proposant un changement temporel, peuvent créer une impression transitoire de rupture du fil narratif.

Dans Zeit für die Bombe, il faut pourtant souligner que beaucoup d'hyperliens sont insérés à la fin d'un paragraphe. Contrairement à certaines hyperfictions comme Afternoon a story [1993], l'activation d'un hyperlien n'est par ailleurs jamais fatale : le lecteur peut toujours revenir en arrière après avoir activé le texte relié, et continuer la lecture du texte géniteur. Faisant passer le lecteur parfois à plusieurs reprises par la même page, 
l'hypertexte de Zeit für die Bombe encourage aussi ce genre de pratiques, lors desquelles le lecteur met en perspective textes géniteurs et textes reliés afin d'évaluer leur contiguïté temporelle, et interprète ensuite les lieux d'indétermination qui résistent.

\section{Hyperlien « successif »}

Le potentiel d'action de certaines relations entre texte géniteur et texte relié dans Zeit für die Bombe peut être appelé « successif ». Le texte géniteur raconte un événement ; l'activation du texte relié permet de découvrir sa suite immédiate. Cet hyperlien préfigure un lecteur désireux de découvrir les épisodes d'une histoire dans l'ordre, alignés les uns après les autres sur la flèche du temps. Même s'il est évidemment impossible de refléter le réel avec tous ses détails et micro-événements, l'hyperlien successif peut provoquer une impression de cohérence unidirectionnelle, potentiellement associée au temps de la vie : un peu comme si les événements se déroulaient en direct, sous les yeux du lecteur.

Tous les hyperliens posés sur le signe « >»» dans Zeit für die Bombe annoncent une telle suite temporelle. Ce signe renvoyant à la flèche du temps, ne serait-ce que par son graphisme, est souvent inséré à la fin d'un paragraphe et peut susciter l'attente d'une suite immédiate. Vers la fin du récit, ce signe est inséré à la marge de textes de plus en plus courts, soutenant le caractère inexorable des événements qui se précipitent vers l'issue tragique.

Dans l'un des épisodes de Zeit für die Bombe ${ }^{16}$, Iwan, l'ex-ami malheureux de Veronika, se retrouve dans un hôpital psychiatrique. Comme Iwan travaille dans cet hôpital en tant qu'étudiant, l'un des médecins décide de le faire sortir. Lorsque le lecteur active le mot "schleussen " (faire sortir) affecté d'un hyperlien, il découvre la suite immédiate du périple d'Iwan après sa sortie de l'hôpital. Cette suite chronologique est couplée à une délocalisation (Iwan erre dans le noir, voir figure 14) et à un changement de focus (le lecteur n'est plus plongé dans la pensée du médecin, mais découvre l'errance d'Iwan).

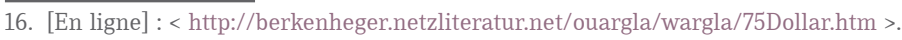




\section{Hyperlien « analeptique »}

Tout lecteur de textes narratifs est habitué à ce que le récit ne présente pas uniquement une suite chronologique des événements, mais l'invite à explorer des sauts en arrière sur la flèche temporelle de l'histoire racontée. Les « analepses » font partie intégrante de l'horizon d'attente, à partir $\mathrm{du}$ moment où le lecteur accepte que le texte narratif construit un monde et manipule librement la charpente de celui-ci. D’éventuelles annonces, ainsi que l'emplacement de l'hyperlien dans le texte géniteur peuvent préparer le terrain au saut temporel.

L'un des épisodes de Zeit für die Bombe décrit les retrouvailles entre Vladimir, l'actuel ami de Veronika et propriétaire potentiel de la bombe, et la jeune fille ${ }^{17}$. Ils font l'amour. Le texte commente : Veronika est « de retour » (je traduis les extraits de Zeit für die Bombe de l'allemand pour plus de clarté). L'expression « de retour » renvoie potentiellement à l'idée que Veronika s'est déjà trouvée dans cette chambre avant la scène racontée, et qu'elle a déjà fait l'amour avec Vladimir. L'activation de l'hyperlien déclenche d'abord l'affichage d'une " page transitionnelle », sur laquelle le narrateur demande au lecteur s'il veut vraiment " savoir » (voir les réflexions sur la " métalepse » plus loin). Le texte sur cette page transitionnelle anticipe donc sur les motivations qui ont potentiellement amené le lecteur à manipuler l'hyperlien. Le texte relié confirme cette attente potentielle : il propose au lecteur de découvrir les épisodes précédents de l'histoire d'amour entre Veronika et Vladimir.

Dans une cabine téléphonique, Blondie, une autre maîtresse de Vladimir, et son frère essaient de joindre Vladimir ${ }^{18}$. Alors que le frère est intéressé par le sort de la bombe, Blondie veut se renseigner sur une lentille de contact qu'elle a oubliée chez son amant. L'activation de l'hyperlien sur "lentille de contact ", inséré dans le discours direct de Blondie, provoque l'affichage d'un texte relié qui met le focus sur Vladimir, délocalisant potentiellement l'action dans l'appartement de celui-ci tout en créant une légère analepse : le lecteur peut découvrir ce que Vladimir a fait dans sa chambre juste avant que le téléphone sonne.

Sur la dernière page du récit ${ }^{19}$, le lecteur apprend que Veronika, dans le coma depuis l'explosion effective de la bombe qu'elle avait amenée à Moscou, rêve encore et encore de sa folle journée passée entre la gare et l'appartement de Vladimir. «Et elle recommença la recherche des débris

17. [En ligne] : < http://berkenheger.netzliteratur.net/ouargla/wargla/84Dollar.htm\#3Cents >.

18. [En ligne] : < http://berkenheger.netzliteratur.net/ouargla/wargla/91Dollar.htm >.

19. [En ligne] $:<$ http://berkenheger.netzliteratur.net/ouargla/wargla/77Dollar.htm >. 
épars et rouges depuis le début ", conclut le narrateur. Une activation de cet hyperlien permet de revenir en arrière sur la flèche du temps de l'histoire, et de parcourir de nouveau les événements qui se sont passés avant que Veronika tombe dans le coma.

Même si le potentiel d'action analeptique de ces hyperliens confirme les attentes suscitées par le contexte, il anticipe sur un lecteur prêt à effectuer une lecture suffisamment exhaustive des textes sur les pagesécran pour percevoir cette contiguité.

\section{Hyperlien « proleptique »}

L'hyperlien à potentiel d'action " proleptique » relie un texte géniteur à un texte relié qui propose un saut vers un futur plus lointain de l'histoire racontée. Des annonces dans le texte géniteur, ainsi que l'emplacement de l'hyperlien préparent éventuellement le terrain à ce saut en avant. Contrairement à l'hyperlien analeptique, qui est également fréquent dans le discours informatif et argumentatif, l'hyperlien proleptique est caractéristique du discours narratif de fiction, qui ne renvoie pas vers un réel supposé objectivable, et peut donc " anticiper " sur l'avenir des lieux et personnages.

Sur l'une des pages-écran de Zeit für die Bombe, le texte décrit le passage de cinq médecins de l'hôpital psychiatrique dans un couloir et relate leurs discussions ${ }^{20}$. Le " cinquième » des médecins éclate de rire lorsque son collègue évoque un homme fraîchement interné qui refuse de lâcher sa valise. L'activation de l'hyperlien sur " cinquième " provoque un changement de focus et de lieu, et permet de découvrir ce que le médecin fait dans un pub, plusieurs heures après la discussion avec ses collègues. Un lieu d'indétermination peut éventuellement émerger de ce saut temporel important qui n'est pas annoncé dans le texte géniteur.

\section{Hyperlien « synchronisant »}

Certains textes narratifs proposent de découvrir des événements qui se déroulent parallèlement. Sur une page papier, ces événements s'affichent les uns après les autres. Le potentiel d'action d'un hyperlien que je propose d'appeler «synchronisant » peut renforcer l'impression de synchronicité parce que les événements parallèles sont exposés sur des pages-écran

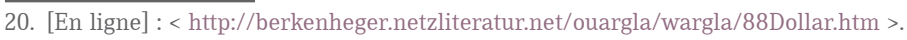


différentes - même si le lecteur est toujours tenu de les lire l'une après l'autre pour actualiser ce potentiel d'action. Cet hyperlien préfigure un lecteur désireux de découvrir différents focus, différentes perspectives sur les événements qui ont lieu lors d'un même laps de temps. Des annonces éventuelles ainsi que l'emplacement de l'hyperlien dans le texte géniteur peuvent anticiper sur la synchronicité des événements racontés.

Après leurs retrouvailles, Veronika et Vladimir font l'amour et, dans leur précipitation, renversent le téléphone ${ }^{21}$. Il sonnera occupé pendant un long moment. L'activation de l'hyperlien sur " téléphone " provoque l'affichage d'un texte relié décrivant ce qui, au même moment, se passe dans un $p u b$ où un jeune homme essaie désespérément de joindre Vladimir. Si le lecteur active l'hyperlien affecté à " signal occupé » sur cette page reliée, le texte décrivant la scène d'amour entre Vladimir et Veronika s'affiche de nouveau. Le lecteur pourrait tourner plusieurs fois en rond entre ces deux pages, adoptant une pratique compulsive qui renvoie potentiellement aux gestes du jeune homme composant frénétiquement le numéro de téléphone de Vladimir.

\section{Figures de la lecture « topologique »}

Les textes reliés aux hyperliens dans Zeit für die Bombe proposent souvent non seulement un saut temporel, mais un changement de lieu par rapport à celui évoqué dans le texte géniteur, faisant émerger des figures de la lecture que j’appelle « topologiques ».

\section{Hyperlien « délocalisant »}

L'hyperlien " délocalisant » invite le lecteur à changer de lieu dans l'histoire racontée. Ce potentiel d'action n'est pas réservé à l'hyperfiction : il peut également émerger de la relation entre texte géniteur et texte relié dans les discours informatif ou argumentatif. L’annonce éventuelle du changement de lieu dans le texte géniteur peut atténuer le risque d'un manque de contiguité.

Sur les premières pages-écran de Zeit für die Bombe, le lecteur assiste à l'arrivée de Veronika à Moscou ${ }^{22}$. Au lieu d'être accueillie par Vladimir, elle tombe dans les bras d'Iwan, son ex-ami, en descendant du train. Elle

21. [En ligne] : < http://berkenheger.netzliteratur.net/ouargla/wargla/84Dollar.htm\#3Cents >.

22. [En ligne] : < http://berkenheger.netzliteratur.net/ouargla/wargla/94Dollar.htm\#2Cents >. 
s'enfuit et prend un taxi. Assise dans la voiture, elle regarde les rues de Moscou défiler à travers la vitre ; la ville est décrite selon la perspective de Veronika, qui reconnaît soudainement une rue et se demande si « elle ne venait pas juste de passer à côté de la chambre de Vladimir ». Compte tenu de la position de l'hyperlien, le lecteur peut s'attendre à entrer effectivement dans cette chambre en cliquant. Le texte relié confirme ces attentes, permettant de découvrir ce qui se passe au même moment dans la chambre de Vladimir. La délocalisation se trouve couplée à un changement de perspective qui préserve l'unité du temps.

Figures de la lecture « dialogique »

Dans les textes journalistiques analysés plus haut, j'ai identifié des hyperliens qui potentiellement déplacent le focus entre texte géniteur et texte relié. Les figures de la lecture "dialogique » anticipent sur un lecteur qui accepte de mettre en perspective différents points de vue. Dans le texte narratif, ces figures diversifient les points de vue sur un personnage, un événement ou un lieu.

\section{Hyperlien « déplaçant le focus »}

À travers l'hyperlien « déplaçant le focus », le lecteur est invité à suivre un déplacement de focus de l'externe vers l'interne, ou inversement. Le lecteur peut découvrir dans le texte relié les pensées d'un personnage qui, dans le texte géniteur, a été décrit seulement à travers ses faits et gestes (suivant la terminologie de Gérard Genette [1983, 50], il s'agit d'un passage de la «focalisation externe » vers la «focalisation interne »).

Le lecteur de Zeit für die Bombe découvre rapidement que Vladimir a deux maîtresses : Veronika et Blondie. Dans l'un des épisodes ${ }^{23}$, Blondie décide de quitter l'appartement de Vladimir après avoir fait l'amour avec lui. Elle descend les escaliers, entre brièvement en collision avec Veronika qui lui est inconnue, et part " ailleurs ". Le texte relié à cet hyperlien propose non seulement la suite chronologique du déplacement de Blondie, mais une plongée dans ses souvenirs de jeunesse.

Le focus peut également se déplacer d'un personnage vers un autre. Le lecteur découvre dans ce cas les paroles prononcées, les actions ou les événements impliquant un autre personnage que celui qui avait été

23. [En ligne] : <http://berkenheger.netzliteratur.net/ouargla/wargla/78Dollar.htm >. 
focalisé dans le texte géniteur. Arrivée en taxi devant l'immeuble de Vladimir, Veronika jette son porte-monnaie au " chauffeur », l'invitant à se servir ${ }^{24}$. En activant l'hyperlien sur chauffeur, le lecteur découvre les pensées du chauffeur de taxi plusieurs heures après (potentiel d'action proleptique), lorsqu'il rentre chez lui. Le lecteur s'attend peut-être bien à un changement de focus en cliquant sur " chauffeur "; une prolepse aussi importante, en revanche, peut éventuellement le surprendre et provoquer l'émergence d'un lieu d'indétermination.

Je reviens à cet autre épisode de Zeit für die Bombe lors duquel cinq médecins discutent dans le couloir d'un hôpital psychiatrique ${ }^{25}$. Un médecin raconte donc le cas d'un homme qui vient d'être interné parce qu'il refuse de lâcher sa valise. Le mot "valise ", dans le discours direct du médecin, est affecté d'un hyperlien. Ayant parcouru les pages précédentes de cette hyperfiction, le lecteur se doute bien que l'homme à la valise n'est autre qu'Iwan, qui voulait récupérer Veronika à la gare de Moscou et se retrouve avec la valise de celle-ci sur les bras. Lorsque le lecteur active l'hyperlien sur "valise ", le focus se déplace vers Iwan dans la chambre d'hôpital, qui hésite à ouvrir la valise. Iwan l'ouvre, découvre la bombe et l'active. C'est maintenant le narrateur qui s'adresse à son personnage (un cas de "métalepse ", voir explications plus loin) et l'incite à se poser, entre autres, la question de savoir " où est Veronika ". L'activation de l'hyperlien sur "Veronika » permet au lecteur de découvrir, dans le texte relié, ce que Veronika fait pendant ce temps dans la chambre de Vladimir. Le potentiel d'action de cet hyperlien est donc synchronique tout en déplaçant le focus.

Selon les explorations déjà effectuées par le lecteur, un lieu d'indétermination peut néanmoins émerger entre les répertoires du texte géniteur et du texte relié : le lecteur ne sait pas forcément comment Veronika est arrivée dans l'appartement de Vladimir, et comment les retrouvailles se sont passées. La scène d'amour elle-même, suggérée dans le texte relié à travers la position allongée de Veronika et son état d'épuisement, reste à imaginer si le lecteur n'a pas encore lu les textes décrivant ces retrouvailles.

24. [En ligne] : < http://berkenheger.netzliteratur.net/ouargla/wargla/74Dollar.htm\#10Cents >.

25. [En ligne] : < http://berkenheger.netzliteratur.net/ouargla/wargla/88Dollar.htm >. 
Figures de la lecture « informationnelle»

Dans la partie précédente consacrée au discours informatif et argumentatif, certaines figures de la lecture ont été appelées « informationnelles », parce qu'elles répondent à la présomption d’information avec laquelle beaucoup de lecteurs abordent le texte numérique. Ces figures existent également dans le discours narratif, où elles étoffent l'univers créé par la fiction en définissant un lieu, en fournissant des compléments d'information ou des explications aux agissements des personnages. Il est tout à fait imaginable qu'un hyperlien inséré dans une hyperfiction renvoie à Wikipédia pour la définition d'un terme technique : dans ce cas, l'hyperlien peut créer un " effet de réel » tout à fait troublant. Dans Zeit für die Bombe, les figures de la lecture informationnelle répondent aux besoins d'informations ou d'explications du lecteur sans jamais toucher à ces barrières : le site ne contient aucun lien extro-projectif. Le lecteur évalue donc la contiguïté entre texte géniteur et texte relié non pas suivant des critères de vérité factuelle, mais de vraisemblance.

\section{Hyperlien « explicatif »}

Comme dans un texte journalistique, l'hyperlien explicatif permet au lecteur de découvrir pourquoi un personnage agit ainsi, pourquoi des événements se déroulent de cette manière. Proposant des réponses vraisemblables aux questions logiques que le lecteur se pose lors de la lecture, ce potentiel d'action a souvent tendance à confirmer ses attentes, d'autant plus si des annonces ou l'emplacement de l'hyperlien dans le texte géniteur suscitent déjà un désir d'explications.

Dans le premier épisode de Zeit für die Bombe ${ }^{26}$, Veronika arrive donc à Moscou et, en sortant du train, tombe sur son ex-ami Iwan, qui la débarrasse de sa valise. Veronika prend la fuite, et avant qu'Iwan puisse réaliser ce qui lui arrive " elle était déjà partie ». Si le lecteur active l'hyperlien sur " partie », il découvre après quelques pages transitionnelles la suite chronologique de l'histoire, et reçoit grâce à un changement de focus des explications du comportement de Veronika : elle est déçue parce qu'elle s'attendait à être accueillie par un autre homme.

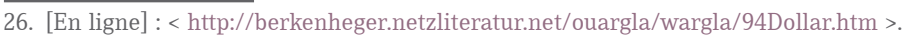


Figures de la lecture « immersive »

Dans certains épisodes de Zeit für die Bombe, le lecteur n'est pas seulement invité à plonger dans l'histoire de Veronika, de Vladimir et d'Iwan en lisant. Il peut parfois s'adonner à l'impression d'agir physiquement sur les objets évoqués, ou d'observer leur mouvement dans l'espace comme s'il s'agissait des choses évoquées. Les couplages entre le texte à lire et les gestes ou les mouvements préfigurent, dans ce cas, des pratiques de lecture immersives.

\section{« Kiné-gramme »}

J’ai proposé d'appeler « kiné-gramme » le procédé rhétorique fondé sur un couplage entre le potentiel d'action d'un texte et celui d'une unité sémiotique de la manipulation, dans lequel l'irradiation iconique est poussée à l'extrême de sorte que se crée un simulacre de référent. Ce procédé n'est pas réservé à l'hypertexte de fiction, même s'il y est plus fréquent que dans le discours informatif ou argumentatif.

Dans l'un des passages de Zeit für die Bombe que j'ai commencé à analyser au premier chapitre, Iwan hésite devant la valise ouverte s'il doit ou non déclencher la bombe. Le narrateur commente : " Alors moi je comprends Iwan : est-ce que nous n'avons pas tous tendance à vouloir toujours appuyer sur quelque chose, cliquer quelque part, afin de déclencher des événements sans grand effort ? C'est en effet la plus belle chose au monde. Iwan, fais-le enfin, appuie sur le petit interrupteur ! »27

Ce passage est d'abord marqué par un procédé exploré dans d'autres textes de fiction, qu'ils soient publiés sur papier ou sur dispositif numérique : il s'agit de la métalepse, dont le potentiel d'action est fondé sur la transgression entre le niveau du récit et le niveau de l'histoire racontée. Le narrateur s'adresse aux personnages, commente leurs actions, se montre parfois omniscient, parfois hésitant, parfois ignorant, leur donne des conseils ou les met devant des alternatives.

Dans l'extrait cité, le narrateur s'adresse à Iwan et l'incite à accomplir l'activation de la bombe. Dans d'autres épisodes de Zeit für die Bombe, le narrateur s'adresse au lecteur en anticipant sur ses réactions, en lui posant des questions, en l'exhortant, voire en l'impliquant directement dans l'histoire racontée en tant que personnage : il lui apprend qu'il sera condamné

27. [En ligne] : < http://berkenheger.netzliteratur.net/ouargla/wargla/96Dollar.htm\#5Cents >. 
à faire la plonge dans un $\operatorname{bar}^{28}$. Jorge Luis Borges $[1957,85]$ résume ainsi le trouble du lecteur provoqué par la métalepse : " De telles inventions suggèrent que si les personnages d'une fiction peuvent être lecteurs ou spectateurs, nous, leurs lecteurs ou spectateurs, pouvons être des personnages fictifs ». Dans le roman papier Tristram Shandy de Laurence Sterne [1760], le narrateur sollicite par exemple l'intervention du lecteur pour aider le personnage Mrs Shandy à regagner son lit. Vladimir Nabokov, cité par Susanne Berkenheger dans une interview comme l'un des auteurs qui l'ont le plus inspirée dans sa pratique d'écriture [Simanowski, 2000], a également expérimenté la métalepse dans beaucoup de ses romans.

Le potentiel d'action métaleptique peut être souligné et confirmé par l'intervention physique du lecteur d'une hyperfiction, car le lecteur effectue vraiment certains gestes de manipulation physiques. Si, chez Laurence Sterne, le lecteur ne peut que mentalement pousser Mrs Shandy vers son lit, il semble pouvoir concrètement précipiter l'arrivée du taxi dans Zeit für die Bombe en cliquant sur l'hyperlien « Taxi, taxi », même si ses gestes n'influencent pas le cours de l'histoire prescrite par l'auteur et vécue par le personnage de fiction Veronika ${ }^{29}$.

Je reviens à l'exemple de l'hyperlien posé sur " appuie sur le petit interrupteur ». L'unité sémiotique de la manipulation " activer ", renvoyant potentiellement à des objets qui s'activent effectivement par un enchaînement rapide d'appui et de relâchement, se trouve dans cet énoncé couplé au mot « interrupteur ", un objet qui s'active par un appui et un relâchement bref. L'injonction du narrateur s'adresse à Iwan, mais l'activation de l'hyperlien est effectuée par le lecteur qui « appuie »geste auquel le verbe dans l'énoncé renvoie également.

La forme-modèle pro-référentielle irradie donc potentiellement le mot " interrupteur » jusqu'à le transformer, au moins transitoirement, en simulacre de référent : le lecteur peut avoir l'impression d'appuyer sur la chose " interrupteur », et non pas seulement sur le mot. L'irradiation iconique provoque ainsi, comme plusieurs lecteurs l'ont fait remarquer (voir relevé des traces de réception plus haut), potentiellement un effet de présentification qui est renforcé par le présent de l'injonction adressée à Iwan.

Même si ce couplage entre texte et forme-modèle pro-référentielle relève donc du kiné-gramme, le simulacre de référent peut se fissurer à plusieurs niveaux, et faire émerger des lieux d'indétermination. D'une

28. [En ligne] : < http://berkenheger.netzliteratur.net/ouargla/wargla/73Dollar.htm >.

29. [En ligne] : < http://berkenheger.netzliteratur.net/ouargla/wargla/86Dollar.htm >. 
part, le texte et sa forme-modèle pro-référentielle relèveront toujours de systèmes sémiotiques fondamentalement différents. D'autre part, l'injonction dans le texte géniteur s'adresse à Iwan, et non pas au lecteur ; la page transitionnelle qui apparaît après l'effectuation du geste, affichant l'énoncé "Iwan appuya », peut confirmer certaines attentes du lecteur tout en attribuant la responsabilité de l'activation de la bombe à Iwan - et non pas au lecteur, qui a pourtant effectué un geste d'activation. Enfin, cet énoncé restitue l'événement dans le passé. Le procédé même du simulacre se trouve donc jusqu'à un certain point dévoilé.

Ce kiné-gramme préfigure un lecteur prêt à s'immerger dans l'histoire racontée en allant jusqu'à devenir physiquement actif, et qui accepte en même temps que le texte lui rappelle sa nature sémiotique ancrée dans le dispositif. Les kiné-grammes dans Zeit für die Bombe sont tous marqués par cette fissure plus ou moins profonde, qui les fait glisser vers la catégorie des « kiné-tropes » (voir figures de la lecture déviative).

\section{« Ciné-gramme »}

J'ai proposé d'appeler « ciné-gramme » un procédé rhétorique fondé sur un couplage entre le potentiel d'action d'un texte et le potentiel d'action d'une unité sémiotique temporelle, dans lequel l'irradiation iconique est poussée à l'extrême de sorte que se crée un simulacre de référent. Après avoir activé l'hyperlien sur " appuie sur le petit interrupteur ", une page transitionnelle restitue d'abord l'événement au niveau de l'histoire racontée : "Iwan appuya ». Le texte relié confirme ensuite que la bombe a été activée. Dans l'énoncé " et la *bombe* faisait tic-tac » qui s'affiche en rouge sur fond noir (voir figure 15), le mot « bombe » clignote rapidement et se trouve donc couplé à l'unité sémiotique temporelle " obsessionnel » (voir explications sur les unités sémiotiques temporelles au chapitre I).

Le mot « bombe " renvoie à l'idée d'un engin explosif. Certaines bombes se caractérisent en effet par un son de tic-tac lorsque leur compte à rebours a été activé. L'unité " obsessionnel » renvoie potentiellement à des idées d'urgence, de danger et de stress. À première vue, le mouvement dans Zeit für die Bombe non seulement confirme, mais encore imite le potentiel d'action mobilisé par le texte, et pourrait donc bien être considéré comme un « ciné-gramme » (ciné comme cinétique). 
Figure 15. Extrait de Zeit für die Bombe
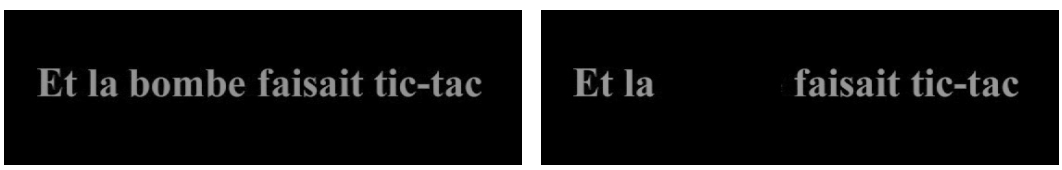

Une fois l'énoncé « appuie sur le petit interrupteur » activé, le mot « bombe » clignote à un rythme rapide dans un contexte stable.

Le simulacre, fondé sur le caractère iconique du mouvement de clignotement, est pourtant à la fois affirmé et fissuré. La séquence animée « et la *bombe* faisait tic-tac » fait émerger une oscillation entre ressemblance et différence, qui joue sur l'iconicité de l'onomatopée " tictac », et le clignotement qui attribue au « tic-tac » la fréquence requise tout en imitant le son par un effet visuel silencieux, complètement étranger à cette bombe spécifique. Un lieu d'indétermination supplémentaire peut se creuser entre le tic-tac suggéré par le passé statique du verbe, et le mouvement visuel qui se répète encore et encore dans le présent, et faire glisser ce couplage vers la catégorie du " ciné-trope ».

Figures de la lecture « déviative »

Dans certains couplages entre le texte et les gestes ou les mouvements, des lieux d'indétermination se creusent alors. La lecture immersive se trouve à la fois encouragée et mise en question.

\section{Kiné-trope}

Après avoir fait l'amour, Veronika veut quitter l'appartement de Vladimir pour retrouver la bombe, mais prend la " mauvaise porte ${ }^{30}$. Le geste d'activation de l'hyperlien sur « mauvaise porte » renvoie potentiellement au geste de pousser une porte, transformant le mot « porte » en simulacre de référent. L'adjectif " mauvaise » annonce une erreur possible, mais qui peut se trouver confirmée seulement après l'activation du texte relié, troublant alors le simulacre de référent. Après l'activation de l'hyperlien s'affiche d'abord un message d'erreur « 404 » qui renvoie explicitement au dispositif numérique. Il est remplacé par un texte revenant à l'histoire racontée et décrivant la situation de Veronika : elle se

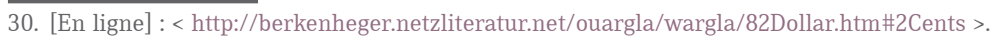


demande comment sortir de là, et soudainement voit une souris disparaître dans un petit trou.

Aucun hyperlien ne se détache visuellement du fond de cette pageécran. S’agit-il d'un «cul-de-sac »? Le lecteur pourrait le croire. S’il commence à scruter le fond de la page-écran en mobilisant l'unité sémiotique « mouvoir » [selon la terminologie proposée par Serge Bouchardon, 2011], il peut pourtant repérer un hyperlien caché. De nouveau, son geste renvoie donc potentiellement à celui effectué par Veronika, qui tâtonne à la recherche d'une sortie. L'hyperlien invisible constitue alors une formemodèle pro-référentielle qui rappelle le «trou de souris » au niveau de l'histoire racontée. La " souris » renvoie pourtant aussi aux caractéristiques du dispositif et trouble potentiellement la lecture immersive, lors de laquelle le lecteur est censé oublier au moins transitoirement le dispositif. Je propose de parler de nouveau de « kiné-tropes » pour caractériser ces couplages entre textes et gestes.

\section{Ciné-trope}

D’autres lieux d'indétermination s'ouvrent dans Zeit für die Bombe entre texte et unités sémiotiques temporelles, que je suggère d'appeler « ciné-tropes».

Sur l'une des dernières pages du récit ${ }^{31}$, la phrase «*Le temps explose en mille éclats* » se trouve couplée à un clignotement obsessionnel. Un lieu d'indétermination particulièrement troublant peut émerger de ce couplage, dans la mesure où une explosion n'est que très improprement imitée par un clignotement. Le lecteur peut interpréter ce manque de contiguïté en ayant recours au contexte du couplage. S'y affiche en outre l'énoncé "Sauve qui peut ». Il serait alors possible d'en déduire que le « danger » de l'explosion est imminent, mais que celle-ci est encore comme " suspendue » dans le temps, donnant aux personnages (et au lecteur) une dernière chance de s'échapper.

Le tableau ci-contre liste encore une fois les procédés rhétoriques de l'hyperlien et de l'animation textuelle, et les figures de la lecture qu'ils font émerger.

31. [En ligne] $:<$ http://berkenheger.netzliteratur.net/ouargla/wargla/1Dollar.htm $>$. 
Tableau 13. Figures de la lecture de l'hyperlien et de l'animation textuelle et leurs procédés

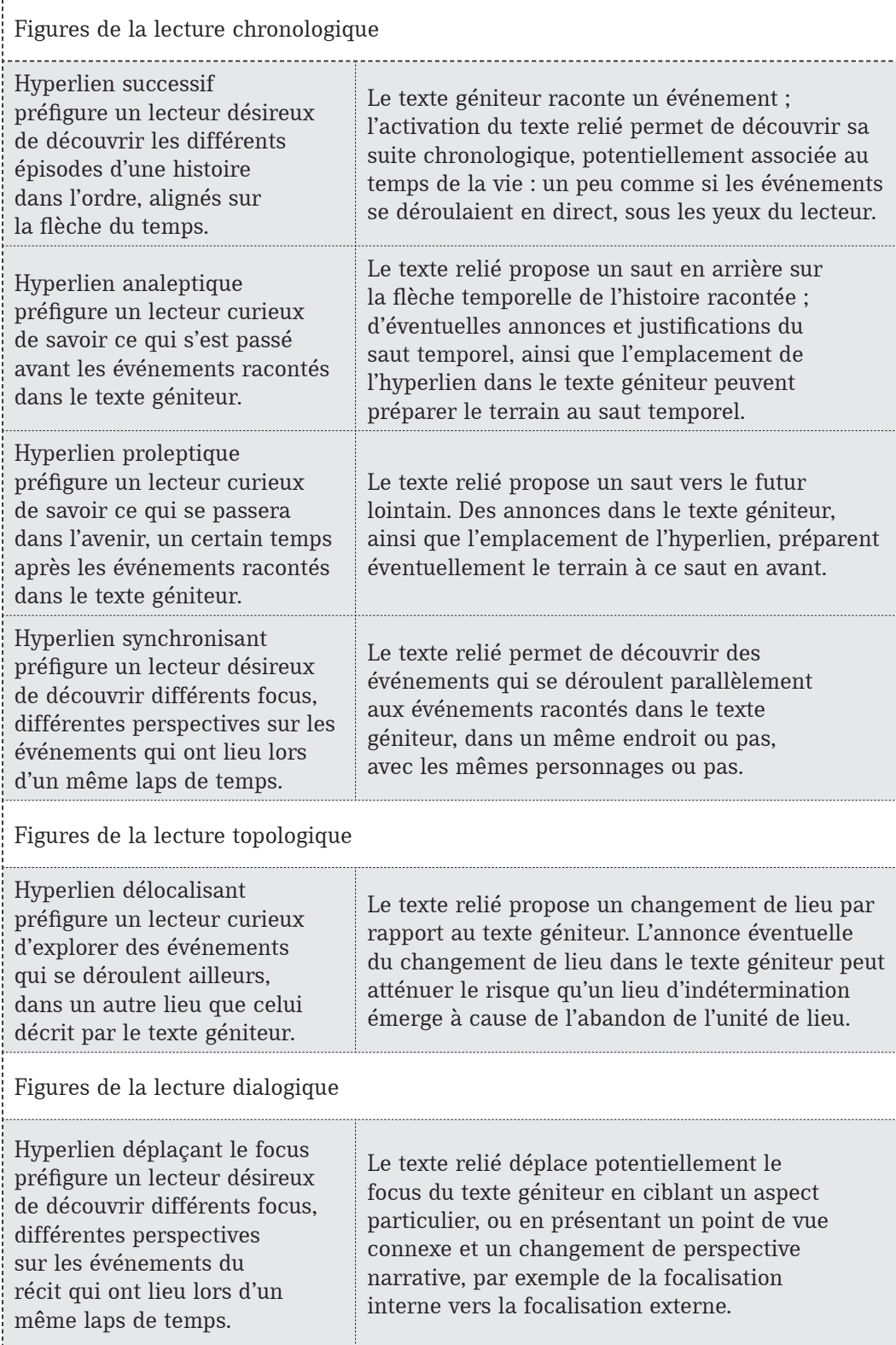


Figures de la lecture informationnelle

Hyperlien explicatif

peut répondre aux attentes

de lecteurs cherchant des

compléments d'information.
Le texte relié décrit les circonstances

d'un événement, l'explique en avançant

des données factuelles ; forte contiguïté

causale avec le texte géniteur.

Figures de la lecture immersive

Kiné-gramme

le couplage entre texte et

forme-modèle pro-référentielle

préfigure un lecteur prêt

à s'immerger, du moins

transitoirement, dans un texte

L'irradiation iconique apportée par

l'unité sémiotique de la manipulation

donne l'impression que le texte se

transforme en simulacre de référent.

devenu simulacre de référent.

Ciné-gramme

le couplage entre texte et

forme-modèle pro-référentielle

préfigure un lecteur prêt

à s'immerger, du moins

transitoirement, dans un texte

devenu simulacre de référent.

Figures de la lecture déviative

Kiné-trope

préfigure un lecteur prêt à

accepter l'oscillation entre

immersion et distance.

Ciné-trope

préfigure un lecteur prêt à

accepter l'oscillation entre

immersion et distance.
L'irradiation iconique de l'unité sémiotique de la manipulation agit sur le texte. Un décalage persiste néanmoins, entre les potentiels d'action du texte et de la forme-modèle pro-référentielle, et trouble l'immersion.

L'irradiation iconique de l'unité sémiotique temporelle agit sur le texte. Un décalage persiste néanmoins, entre les potentiels d'action du texte et de la forme-modèle pro-référentielle, et trouble l'immersion.

\section{Proposition d'interprétation d'une hyperfiction}

Les outils présentés dans les pages précédentes aident à circonscrire les potentiels d'action d'une hyperfiction. Comme pour l'article journalistique, je propose ci-contre une synthèse de l'analyse que j'ai effectuée 
page-écran par page-écran ; elle fait émerger les contiguités et les lieux d'indétermination potentiels de Zeit für die Bombe. Elle restitue ainsi une expérience de lecture résolument intensive, qui s'est effectuée le stylo (numérique) à la main, et qui avait comme but à la fois l'exhaustivité et la précision. Elle résume cette expérience en établissant des cohérences ; pour cela, les épisodes de l'histoire racontée ont été mis dans un ordre qui n'est pas forcément celui de l'exploration en acte.

Cette étape fait partie de l'interprétation possible de Zeit für die Bombe, qui propose des hypothèses pour meubler les lieux d'indétermination du texte et assurer un certain seuil de compréhension partageable. Je mobilise dans cette étape mes connaissances personnelles du champ de l'hyperfiction, du récit contemporain et des fonctionnalités du dispositif numérique.

Après l'explosion d'une bombe dans une gare à Moscou, la jeune Veronika tombe dans le coma. Sur la dernière page du récit, le lecteur apprend que tout le réseau de parcours narratifs, interconnectés par hyperliens, reflète donc potentiellement la conscience de la jeune femme, qui tourne autour de certaines décohérences de sa mémoire. Comment est-elle arrivée dans cette situation?

Zeit für die Bombe est le récit d'une folle journée d'hiver, durant laquelle plusieurs personnages se croisent et se perdent dans les rues de Moscou, ses gares, un hôpital psychiatrique, un appartement délabré et un pub lugubre. L'été précédent, Veronika a eu une aventure avec Vladimir. Celui-ci a demandé à la jeune femme de transporter une bombe lors de sa prochaine visite. "Sauver l'âme russe ", voilà tout ce que le lecteur apprend sur les motivations de Vladimir. Veronika a accepté la mission.

Arrivant à la gare de Moscou, elle s'attend à être accueillie par Vladimir. C'est pourtant Iwan, son ex-ami, étudiant en psychiatrie, qui l'attend à la gare. Veronika tombe directement dans ses bras en sortant du train. Iwan récupère sa valise, par galanterie. Encore sous le choc de ces retrouvailles inattendues, Veronika s'enfuit vers un taxi et dans sa hâte oublie la valise. Iwan reste seul sur le quai de la gare. Par hyperlien, le lecteur a le choix de suivre Veronika ou Iwan dans leurs périples respectifs ; s'il regrette son choix, il peut revenir en arrière, et explorer un parcours alternatif.

Blondie, deuxième maîtresse de Vladimir, son frère impliqué dans le projet de la bombe ainsi qu'un chauffeur de taxi, un patron de pub et un médecin tuteur d'Iwan rejoignent le cercle des personnages. Des pages « transitionnelles », parfois animées, s'intercalent entre les pages porteuses d'hyperliens ; s'affichant et disparaissant de l'écran sans que le lecteur puisse intervenir sur la cadence de défilement, ces formes-modèles 
pro-depréhensives assurent la transition entre les parcours et imposent un rythme de lecture haletant.

Les en-têtes des pages-écran, loin de proposer seulement un résumé ou une annonce du contenu de la fenêtre de lecture, affichent parfois des commentaires du narrateur, et souvent rappellent potentiellement la synchronie des événements. Lorsque le lecteur s'engage pour la première fois dans la lecture de Zeit für die Bombe, certaines en-têtes peuvent lui paraître énigmatiques. La plupart de ces lieux d'indétermination se résolvent potentiellement lorsque toutes les pages-écran ont été explorées.

Si le lecteur décide de "suivre » le départ précipité de Veronika, il apprend qu'elle monte dans un taxi pour rejoindre Vladimir. Après quelques moments d'errance dans les rues de Moscou, Veronika arrive devant l'immeuble de Vladimir. Devant la porte, elle entre en collision avec une jeune fille blonde qui vient de sortir de chez Vladimir après avoir fait l'amour avec lui. Veronika ne sait pas encore qu'il s'agit de Blondie, la deuxième maîtresse de Vladimir. Selon les hyperliens déjà activés, le lecteur le sait, ou il aura la possibilité de l'apprendre plus tard. Il apprendra dans ce cas aussi que Blondie a oublié une lentille de contact chez Vladimir. Certaines allusions à une " poudre blanche » peuvent suggérer que Vladimir n'est pas qu'un poseur de bombes. Cette piste n'est pourtant pas développée.

Après avoir sonné intempestivement à la porte, Veronika entre chez Vladimir. La scène de retrouvailles provoque entre autres la chute du téléphone, qui sonnera occupé pendant un long moment. Vladimir revient au sujet de la bombe. Veronika se souvient de la valise abandonnée dans les bras d'Iwan. Elle ressort précipitamment de l'appartement de Vladimir. Juste avant, elle remarque la lentille de contact.

Selon les hyperliens activés, Veronika commence soit à avoir des soupçons concernant la fidélité de Vladimir, et s'en va furieuse ; soit son sentiment amoureux est encore intact lorsqu'elle arrive devant la gare. Si le lecteur active certains hyperliens, il apprend qu'elle intercepte, à ce moment, une conversation de Blondie et de son frère dans une cabine téléphonique. La jeune fille parle de sa lentille de contact, son frère évoque « la bombe ». Veronika en déduit que les deux personnes sont en conversation avec Vladimir. Si le lecteur n'a pas activé les hyperliens en question, il apprendra peut-être seulement lors d'une deuxième exploration pourquoi Veronika, furieuse, fait demi-tour et revient chez Vladimir pour l'interroger.

Après une violente dispute, les deux se réconcilient sur l'oreiller, et Veronika repart une deuxième fois pour retrouver la bombe. Elle prend cependant la « mauvaise porte ». En activant l'hyperlien sur « mauvaise 
porte », le lecteur arrive sur une page d'erreur « 404 » sur laquelle, au bout d'un court instant, s'affiche la phrase " Où peut-on sortir d'ici, demande Veronika, et voit une petite souris disparaître dans un petit trou ». En scrutant la page avec le curseur, le lecteur peut repérer un hyperlien caché, désigné lors de l'activation comme un " trou à travers l'espace et le temps ".

Sur la page reliée, il découvre comment Veronika revient dans l'appartement de Vladimir et lui demande d'indiquer la «bonne porte ». Si le lecteur active l'hyperlien sur "bonne porte ", il découvre Vladimir en train de faire l'amour avec Blondie. Comment Blondie a-t-elle pu arriver si vite dans l'appartement de Vladimir, alors que Veronika avait passé juste un court instant derrière la " mauvaise porte " ? Même au bout de plusieurs explorations de Zeit für die Bombe, le mystère de ce " trou à travers l'espace et le temps » reste entier. Il constitue un lieu d'indétermination résistant à toute tentative d'établir de la contiguité, et met potentiellement au défi les attentes d'un lecteur habitué à la charpente logicotemporelle du récit traditionnel. Il peut être interprété comme une tentative d'attirer l'attention sur le caractère construit de ce texte de fiction, ainsi que sur ses caractéristiques médiatiques. Cette hypothèse peut être justifiée aussi par le fait que la page, affichant les mots " erreur 404 », contient ellemême une allusion au dispositif numérique.

Veronika repart en tout cas, fermement décidée de quitter Moscou au plus vite. Pendant tout ce temps, Iwan était en possession de la valise. Resté seul sur le quai de gare, il plonge dans le délire. Il est embarqué par des infirmiers et transporté à l'hôpital psychiatrique. Alors que les médecins débattent de son sort et que son tuteur envisage des moyens pour le faire sortir, Iwan, dans sa chambre d'hôpital, hésite à ouvrir la valise de Veronika. Lorsqu'il le fait, il découvre la bombe. Doit-il appuyer sur l'interrupteur?

Le narrateur semble l'y inciter et le lecteur est amené, par le couplage entre l'énoncé « appuie sur le petit interrupteur » et l'unité sémiotique de la manipulation " activer ", à prendre part à l'activation. La page transitionnelle qui suit rappelle pourtant que c'est Iwan qui a appuyé sur le petit interrupteur. Le compte à rebours est lancé : « la *bombe* faisait tic-tac », indique le texte, alors que le mot " bombe » clignote silencieusement sur l'écran. Entre le passé et le présent, entre le mouvement clignotant silencieux et le son décrit du tic-tac, apparaissent des fissures qui à la fois confirment et mettent en question les simulacres de référent créés par les ciné-grammes et kiné-grammes dans Zeit für die Bombe. 
Aidé par son professeur, Iwan sort de l'hôpital. Encore obnubilé par les médicaments, tâtonnant dans le noir qui se reflète dans les formesmodèles de la page-écran sombre, Iwan repère l'entrée d'un pub : le même pub où le frère de Blondie essaie de joindre Vladimir pour échanger sur le problème de la bombe.

Le frère de Blondie essaie de joindre Vladimir depuis le téléphone du $p u b^{32}$. Selon les parcours déjà effectués, le lecteur sait peut-être pourquoi le téléphone sonne occupé chez Vladimir : il fait l'amour avec Veronika. Blondie rejoint son frère et pousse un " haaalloooo " tonitruant ${ }^{33}$. Après avoir activé l'hyperlien posé sur ce cri, le lecteur apprend que Blondie, qui s'était jetée avec effusion sur son frère, a endommagé le téléphone. Le couple s'enfuit du pub et cherche une cabine téléphonique. C'est devant cette cabine que Veronika surprendra leur conversation avec Vladimir.

Si le lecteur a suivi le périple d'Iwan vers le même bar, il peut avoir l'impression de se retrouver à un moment donné sur la même page, avec le cri « haaalloooo » activable en bas de la page-écran. En revanche, l'hyperlien ne mène plus vers le texte décrivant la fuite du couple frère et sœur, mais vers Iwan qui sort du bar suivant les indications de Blondie. Selon le chemin parcouru, le " haaalloooo ${ }^{34}$ hyperlié s'adresse donc soit au frère de Blondie, soit à Iwan : s'ouvre un autre lieu d'indétermination potentiel où la charpente logicotemporelle du récit se fissure.

Alors que la contiguïté entre la plupart des épisodes reliés par des hyperliens préfigure un lecteur prêt à suspendre au moins partiellement son incrédulité face au caractère fictionnel de l'histoire, ce lieu d'indétermination attire de nouveau l'attention sur le caractère construit de la fiction, et sur le dispositif. Sont ainsi préfigurées des pratiques que MarieLaure Ryan a qualifiées de "medium-aware" [2001, 351].

À un moment, le lecteur lui-même est invité par le narrateur à entrer dans le pub en activant un hyperlien. À un autre moment, il est comme « embarqué de force » et contraint de rester dans le pub. Si, dans le texte décrivant la fuite de Blondie et de son frère, il active la question « peutêtre pas vous ? », il se fait « rattraper » par le patron et doit « faire la plonge » à la place du couple. Il est également « condamné » à rejoindre le pub lorsque, lisant les cogitations d'Iwan dans sa chambre d'hôpital, il choisit l'hyperlien posé sur l'énoncé « appeler la police ». Ces effets

32. [En ligne] : < http://berkenheger.netzliteratur.net/ouargla/wargla/84Dollar.htm\#3Cents >.

33. [En ligne] : < http://berkenheger.netzliteratur.net/ouargla/wargla/98Dollar.htm >.

34. [En ligne] : < http://berkenheger.netzliteratur.net/ouargla/wargla/97Dollar.htm >. 
métaleptiques jouent également avec l'immersion du lecteur tout en révélant leur caractère construit, de façon souvent humoristique.

Pendant qu'Iwan s'engage maintenant vers la gare, Blondie et son frère arrivent enfin à joindre Vladimir, qui invite Blondie à récupérer sa lentille de contact. Ils font de nouveau l'amour, mais Veronika les surprend (en passant par le " trou à travers l'espace et le temps ») et s'en va en taxi, furieuse. Le chauffeur de taxi, qui est en fait le père de Blondie, commence à se lasser de ce va-et-vient frénétique des personnages.

Veronika rejoint la gare, Iwan aussi. Iwan cherche Veronika et, sur les indications d'un collègue, s'engage vers le quai. Veronika penche la tête hors de la fenêtre du train et reconnaît Iwan ; pour un bref instant, une réconciliation semble possible ; c'est à ce moment que la bombe explose, tuant Iwan et 32 autres passagers. Veronika tombe dans le coma. Sur la dernière page du récit, le lecteur est invité à recommencer l'exploration.

Dès la première page-écran, l'utilisation de Netscape 3.XX est recommandée au lecteur. Ce navigateur dominait le marché dans les années 1990. Son développement a été arrêté en 2008. L'inaccessibilité du dispositif d'actualisation recommandé a provoqué certains lieux d'indétermination entre les événements de surface prévus par l'auteur, et l'actualisation de l'œuvre en 2013. Les témoignages des premiers lecteurs et de l'auteur permettent de les circonscrire.

Lorsque Veronika est dans le train de retour, le lecteur est invité à activer un hyperlien sur " hall de gare ». Le témoignage de Nina Hautzinger [1999] évoque l'existence d'une fenêtre pop-up dans laquelle on pouvait lire «Que dalle. La bombe explose sur $\mathrm{OK}$ », avec le mot « OK » activable. Cette deuxième étape d'activation de la bombe, et ce deuxième renvoi vers le dispositif ont aujourd'hui disparu de la surface observable de l'œuvre.

Un autre exemple : selon Roberto Simanowski [1999], on pouvait, lors de la parution de Zeit für die Bombe, changer de page non seulement en activant les hyperliens, mais aussi en appuyant sur la touche " entrée ». L'auteur a interprété cette possibilité comme le reliquat d'une présentation linéaire du texte, plutôt caractéristique du livre papier. Cette possibilité de feuilletage a également disparu.

Comme le souligne Hans Robert Jauss [2010, 125], une œuvre traverse les époques non pas parce qu'elle pose des questions éternelles ou parce qu'elle donne des réponses atemporelles, mais en raison d'une tension plus ou moins ouverte entre question et réponse, qui relance le dialogue entre l'horizon de l'œuvre et l'horizon d'attente du lecteur. L'analyse de Zeit für die Bombe, mise en perspective avec d'autres traces de lectures, montre comment les 
lieux d'indétermination entre textes géniteurs et textes reliés, entre textes à lire et formes-modèles peuvent se déplacer en fonction des attentes du lecteur. Ils continueront à se déplacer avec l'évolution des pratiques de lecture numériques, et avec la démocratisation des " hypertextes de fiction » pour tablettes [voir les succès éditoriaux d'Actialuna, Storylab...] ${ }^{35}$.

Certains lieux d'indétermination peuvent se dissoudre si le lecteur s'engage dans une pratique de lecture intensive des textes ; d'autres continuent à résister. C'est précisément parce que le potentiel d'action de Zeit für die Bombe ne renvoie pas d'emblée aux paradigmes de la fragmentation et de la décohérence ; c'est parce que la charpente logicotemporelle permet des pratiques de lecture immersives, que les lieux d'indétermination échappant à ces contiguïtés défient fortement les attentes du lecteur.

Le potentiel d'action de Zeit für die Bombe se caractérise par une oscillation entre des formes-modèles pro- et non référentielles, prodepréhensives et pro-intensives, des ciné-grammes, kiné-grammes, cinétropes et kiné-tropes, et des renvois vers le dispositif mettant en question les simulacres de référent. Comme certains romans de Jean Echenoz ou de Pierre Michon, Zeit für die Bombe peut être considéré comme un " médiateur pour temps flottants » [expression empruntée à Blanckeman, 2000] - à condition que le lecteur s'y plonge.

Comme je l'ai montré à travers l'analyse de Zeit für die Bombe, il est tout à fait possible d'intégrer de l'animation textuelle au sein d'un discours narratif. La temporalité, apportée au texte par l'irradiation iconique, peut être mobilisée pour raconter des histoires qui imposent leur rythme de déroulement au lecteur. Je rappelle l'exemple «Et la *bombe* faisait tic-tac ", où le mot *bombe* clignote rapidement. Plusieurs séquences narratives pourraient d'ailleurs s'enchaîner dans cette même animation : imaginons que l'énoncé, au bout d'une phase de clignotement, explose en mille morceaux sur la page-écran, ou qu'il arrête de clignoter.

Est-il pourtant possible d'argumenter avec une animation textuelle, autrement dit : l'iconicité apportée au texte par la forme-modèle du mouvement peut-elle donner des raisons pour ou contre une proposition ? Si l'on réduit l'argumentation à l'étude des moyens par lesquels on appuie une proposition, il est sans doute difficile d'envisager des exemples. Cette difficulté s'explique par le caractère iconique des mouvements en question. Même s'il est possible de circonscrire jusqu'à un certain point leur

35. Actialuna, [en ligne] : < http://www.actialuna.com > Storylab, [en ligne] : < http://www.Storylab.fr/ >. 
potentiel d'action, il ne faut pas oublier que celui-ci « n'est pas un objet pour la pensée consciente », mais plutôt « une forme avec laquelle le corps percevant entre en résonance mimétique » [Meunier, 2006, 137].

À partir du moment où la définition de l'argumentation intègre en revanche le discours persuasif qui fait appel à l'affectif, les procédés rhétoriques de l'animation textuelle font partie intégrante de l'argumentation. Dans le chapitre consacré au corpus journalistique, ce glissement entre discours argumentatif (au sens restreint) et discours persuasif (qui pourrait intégrer une définition plus large de l'argumentation) s'observe dans les exemples de kiné-grammes et de kiné-tropes, qui mettent en œuvre une irradiation iconique apportée par la manipulation physique du texte sur l'interface écranique. Dans l'étude du dernier corpus constitué de bannières publicitaires, c'est l'animation qui soutient la nature persuasive du discours.

\section{FIGURES DE LA LECTURE DANS LE DISCOURS PERSUASIF}

Le marché de la publicité numérique est important ; l'intérêt du lecteur pour la publicité, en revanche, est faible. Il prête une attention réduite à ces éléments qui se trouvent souvent dans la partie périphérique de son champ de vision (voir études citées au chapitre II). Néanmoins, les discours de la publicité numérique ont une influence sur le comportement [voir Anand, Sternthal, 1991]. Didier Courbet [2004, 38] appelle persuasion " sans conscience » ces « effets affectifs et non conscients où l'activité du sujet, non verbalisable, n'amène pas à la construction de significations mais conduit, tout de même, à engrammer des traces mnésiques et à produire des effets comportementaux ».

Plusieurs facteurs pourraient être à l'origine de cette " persuasion sans conscience » : grâce aux couleurs et aux formes, le lecteur reconnaît peut-être une marque sans focaliser son attention sur la bannière. Je partirai ici de l'hypothèse que le caractère iconique des mouvements mobilisés dans les bannières animées agit également sur le lecteur, même si le texte n'est pas déchiffré. Le texte à lire est néanmoins présent dans les exemples sélectionnés. Son potentiel d'action peut donc être défini comme une interaction entre ce texte à lire, et les mouvements.

La focalisation directe ou indirecte, la lecture attentive ou la perception non consciente d'une bannière publicitaire relève de la " décision sémiotique » [Klinkenberg, 1996, 83] du lecteur, qui isole une partie intelligible du monde et en retire une signification. Les auteurs de 
bannières anticipent certainement sur ces deux situations extrêmes de la lecture : celle où la bannière agit sur le sujet sans qu'il y prête attention, et celle où il regarde la bannière de près parce qu'elle l'intéresse. Dans cette deuxième situation, l'anticipation des pratiques de lecture peut aller jusqu'au "partage poétique » de scènes imaginaires, comme l'a formulé Nicole Pignier [2005, 532, voir plus haut].

Avant de présenter les bannières animées retenues pour ce dernier chapitre, voici une brève reprise de la méthodologie déjà amplement présentée et discutée plus haut.

Le texte à lire dans une bannière mobilise des " répertoires » [Iser, 1976 ; 1995] en direction du lecteur : il fait appel à des normes sociales, des croyances, des valeurs partagées, des événements ou personnages supposés connus par le lecteur. Des animations comme le clignotement d'un mot, sa disparition ou son passage de gauche à droite, couplent un déroulement temporel à ce texte à lire. J'ai indiqué plus haut que des mouvements aux caractéristiques visuelles assez différentes peuvent provoquer un effet de sens semblable chez le lecteur : par exemple, lorsque le mot « soldes » est couplé à un clignotement, à un changement de couleur ou à un changement de taille rapides et réitérés (voir modélisations au chapitre I). J'en ai conclu que ce n'est pas chaque micro-phénomène visuel (comme une apparition ou une disparition dans un clignotement), mais plutôt des enchaînements de mouvements qui font potentiellement sens pour le lecteur.

J'ai convoqué les recherches menées par le laboratoire MIM pour donner du fondement à cette idée. Le laboratoire a identifié des unités sémiotiques temporelles (UST) dans la musique ${ }^{36}$ : des enchaînements de sons, que les auditeurs reconnaissent comme des unités de sens grâce à leurs caractéristiques touchant au rythme, à la réitération et à l'intensité. Lorsqu'on écoute des exemples d'unités sémiotiques temporelles sur le site du MIM, et que l'on visualise en même temps des animations qui se déroulent au même rythme, on ne peut qu'être frappé par l'impression de synesthésie. La pulsation sonore rapide de l'« obsessionnel » semble entrer en correspondance étroite avec des mouvements de pulsation visuelle, que ceux-ci se manifestent sur la page-écran par un clignotement, un changement de taille ou un changement de couleur rapide.

Les UST peuvent donc être considérées comme les unités d'une sémiotique temporelle implémentable dans du son, du texte ou de l'image. La

36. Laboratoire Musique et informatique de Marseille, "Les unités sémiotiques temporelles », [En ligne] : < http://www.labo-mim.org/site/index.php?2008/08/22/44-liste-des-19-ust >. 
reconnaissance d'une unité sémiotique temporelle est fondée sur des processus d'intégration et de stabilisation d'expériences antérieures : le lecteur reconnaît un " obsessionnel " parce qu'il a déjà vu clignoter des boutons d'alarme ou des enseignes lumineuses dans son entourage... Le potentiel d'action de l'UST ne se démarque guère de ces référents d'expérience, et renvoie à des idées de stress, d'urgence, de danger et d'excitation. L'icône fait pourtant autrement appel à nos sens que le texte à lire, et la verbalisation de son potentiel d'action doit être effectuée avec prudence. Lorsqu'une unité sémiotique temporelle est couplée à un texte, des signes de nature très différente interagissent sur le même support.

J'ai étudié les textes du corpus journalistique en les appelant « discours informatif et argumentatif ». J'introduis maintenant le corpus de bannières publicitaires et d'annonces commerciales en les qualifiant de " discours persuasifs ». Cette distinction doit être explicitée.

Comme je l'ai indiqué au chapitre I, la rhétorique est souvent définie comme un art du discours persuasif qui inclut l'argumentation. Il serait certes possible de proposer, comme Richard Arcand et Nicole Bourbeau [1995, 126], la distinction suivante entre discours argumentatif et persuasif : " au moyen d'une argumentation rationnelle, un argumentateur produit un discours argumentatif dans le but de convaincre alors que, au moyen d'une argumentation non rationnelle, un persuadeur produit un discours persuasif dans le but de persuader ». Le discours persuasif ferait plutôt appel aux émotions et croyances, alors que le discours argumentatif mobiliserait un raisonnement rationnel. Il est pourtant difficile de concevoir qu'une argumentation rationnelle ne suscite aucune émotion, ou qu'un discours persuasif se passe d'arguments rationnels.

Dans le domaine de la rhétorique visuelle, la distinction entre argumentation et persuasion paraît plus spontanément acceptable. Si l'on définit l'argumentation comme l'étude des moyens rationnels par lesquels on donne des raisons pour appuyer une proposition, il n'est certes pas impossible de repérer des images argumentatives. Dans ce cas, une image donnerait les raisons pour ou contre une proposition au travers des énoncés visuels [voir Roque, 2011]. Je me range néanmoins à l'observation de Marc Bonhomme [2008, 215] qui indique que la rhétorique visuelle, notamment dans la publicité, est souvent mobilisée pour orienter les associations du consommateur de façon persuasive. Marc Bonhomme cite comme exemple une publicité où la forme d'une grappe de raisin et la forme de l'île de Corse se trouvent fusionnées. L'auteur parle d'un " transfert iconique » pour caractériser le couplage de formes. J’ai proposé le terme irradiation 
iconique pour décrire ce même processus dans l'animation textuelle. Dans certaines bannières et annonces commerciales animées s'observe néanmoins un glissement du persuasif vers l'argumentatif, comme je le montrerai plus loin.

\section{Constitution d'un corpus de bannières publicitaires et d'annonces commerciales}

Mon corpus accueille cinq bannières et annonces commerciales. J'ai opté pour ces exemples parce qu'ils mobilisent des textes à lire couplés à une ou plusieurs unités sémiotiques temporelles. Les bannières publicitaires ont été sélectionnées à partir d'une collection publiée sur le site journaldunet.com. Le site les présente sous forme de captures d'écran animées, qui reproduisent la bannière hors contexte. Ce contexte, des sites aux contenus les plus divers, peut être partiellement reconstruit à partir d'indications données par journaldunet. Toutes les bannières renvoyaient au moment de leur publication à un site de marque. Les annonces commerciales ont été capturées au hasard de mes explorations sur Internet ; elles ne constituent pas des bannières apposées au contenu éditorial principal d'une page-écran, mais font partie intégrante de ce contenu. Pour assurer l'homogénéité du corpus, j'ai effectué des captures d'écran animées qui les isolent du contexte de la page-écran. Je donnerai néanmoins des indications sur leur contexte de publication tel que je l'ai expérimenté.

Le premier exemple provient d'une page du site agentdunet.com recensant des casinos en ligne. S'alignent, sur cette page, plusieurs banderoles horizontales. Chaque casino est représenté par deux carrés dans la banderole : le premier donne des informations factuelles comme le nombre de jeux accessibles, la somme minimale de dépôt et une somme offerte lors du premier enregistrement du joueur ; le deuxième carré, juxtaposé au premier, accueille parfois le logo du site en question, parfois des éléments animés. Pour le Casino Glamour, premier site de la liste ${ }^{37}$, c'est la somme mise à disposition lors du premier enregistrement qui occupe une grande partie du carré. « 50 \$ offerts » s'affiche en violet. Un liséré blanc apparaît et disparaît autour de l'énoncé de sorte que se crée un mouvement de pulsation rapide. Au-dessus de l'animation est inséré le logo du casino ; en dessous se trouve l'injonction " Jouez avec notre argent », dédoublée

37. [En ligne] :< http://www.agentdunet.com/casino.html >. 
par l'énoncé « Jouez maintenant » formaté en surbrillance. L'animation tourne en boucle.

Figure 16. Capture d'écran d'un extrait du site Agentdunet
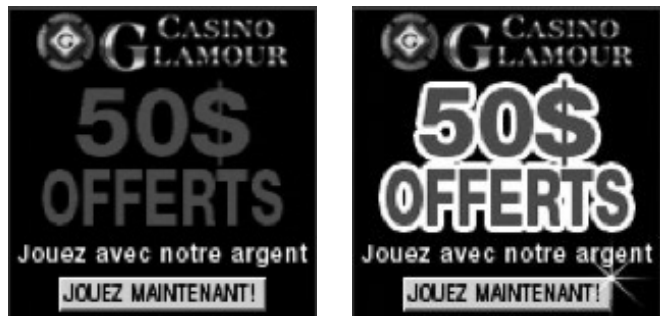

Consultable à l'adresse : < http://www.agentdunet.com/casino.html > (consulté le 15 juin 2012). Un liséré blanc apparaît et disparaît de façon rapide et réitérée autour de l'énoncé « 50 \$ offerts ».

L'exemple suivant est une bannière repérée dans la collection publiée en mai 2008 sur journaldunet ${ }^{38}$. Elle s'inscrit dans une campagne plus vaste, lancée par l'Agence régionale de développement des territoires d'Auvergne autour du thème "Les Urbanophiles ». Déclinée autour de bannières en noir et blanc contenant des animations textuelles, et d'un jeu appelé « l'Urbanette », elle a été conçue par l'agence Singapour ${ }^{39}$ et s'est trouvée insérée entre autres sur les sites cessionpme.com (vente de fonds de commerce et de locaux professionnels), journaldunet.com et societe.com.

Sur la bannière sélectionnée pour le corpus, l'énoncé textuel « Soyez stressé, c'est bon pour la productivité », affiché en blanc avec un liséré noir et en lettres partiellement fissurées, bouge sur une image de fond grise qui renvoie à une route goudronnée. Juste à côté de l'énoncé textuel, le couvercle d'une bouche d'évacuation est visible. L'énoncé textuel vibre plusieurs fois de façon rapide ; la vibration est accompagnée d'un passage de gauche à droite qui finit par faire glisser l'élément textuel hors champ. Juste avant que ce passage de gauche à droite commence, «Conseil $\mathrm{n}^{\circ} 1$ » apparaît à côté de la phrase vibrante. Animé d'abord par un mouvement de flash, il reste stabilisé à l'écran jusqu'à ce que le

38. [En ligne] : < http://www.journaldunet.com/ebusiness/rubriques/creations-pub-selections-mensuelles/080527-selections-pub-mai/1.shtml >.

39. [En ligne] : < http://www.singapour.com >. 
conseil $n^{\circ} 2$ remplace le premier : « Dites bonjour à votre patron, c'est lui qui vous paie ». Puis vient le tour du conseil $n^{\circ} 3:$ : Portez un costume gris, vous serez assorti à la couleur du ciel », toujours animé de la même façon. Vibre enfin la phrase «Quelle chance de travailler en ville » sur le décor gris. À côté s'inscrit et se stabilise l'adresse www.lesurbanophiles. com, et l'énoncé « Cliquez ici » clignotant. L'animation reprend au bout de quelques instants.

Figure 17. Bannière publicitaire publiée dans une collection de journaldunet

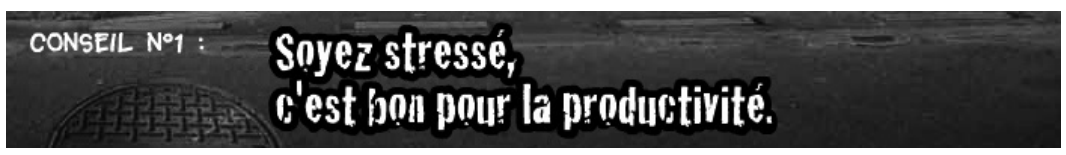

\section{CONSEIL No1: SỌYCZ StreSSQ, c'est bon pour la productivité.}

Consultable à l'adresse : < http://www.journaldunet.com/ebusiness/rubriques/ creations-pub-selections-mensuelles/080527-selections-pub-mai/1.shtml > (consulté le 15 juin 2012). L'énoncé « Soyez stressé, c'est bon pour la productivité » vibre rapidement tout en bougeant vers la droite. L'impression de vibration rapide est impossible à reproduire dans la capture d'écran statique.

Le troisième exemple a été publié dans la collection de février 2009 par journaldunet ${ }^{40}$. Créé par l'agence Fullsix ${ }^{41}$ pour le Club Med, il existe sous deux formats : une bannière longiligne destinée à la partie supérieure des pages-écran, et une bannière plus carrée destinée à la partie droite. Les bannières ont été publiées sur des sites aux contenus les plus divers : des sites immobiliers (avendrealouer.com, lesiteimmobilier.com), des magazines féminins (elle.com, femmeactuelle.com), des portails d'information sportive (eurosport, equipe, laposte, aol, yahoo, capital...).

J'ai choisi comme exemple la version carrée de la bannière. À droite s'affiche le portrait d'un homme souriant. Il porte des lunettes de ski sur lesquelles se reflète une composition de plusieurs images : un skieur en pleine action, deux personnes nageant dans une piscine, un bâtiment

40. [En ligne] : < http://www.journaldunet.com/ebusiness/rubriques/creations-pub-selections-mensuelles/090306-selections-pub-fevrier/3.shtml >.

41. [En ligne] : < http://www.fullsix.com >. 
éclairé juxtaposé à un panorama alpin. Un panorama de montagne s'affiche également à côté du skieur sur le fond gris rosé de la bannière. En haut à gauche s'y superpose le logo du Club Med en blanc, en bas de la bannière s'étale, sur toute la longueur, une banderole rouge sombre. L'énoncé « Le tout compris, c'est... » s'y affiche en blanc, juxtaposé à une petite flèche. Dès que le lecteur effleure la banderole avec le curseur, un expand se déploie à l'intérieur de la bannière et remplace toutes les images par un texte détaillant l'offre commerciale.

Trois énoncés animés apparaissent et disparaissent successivement et lentement, par floutage et défloutage, à côté du visage du skieur : "Partez entre le 10 janvier et le 26 avril 09 », « 200 euros offerts à deux* (*voir conditions sur le site) », " Réservez avant le $1^{\text {er }}$ mars 09 ». L'animation tourne en boucle.

Figure 18. Bannière publicitaire publiée dans une collection de journaldunet
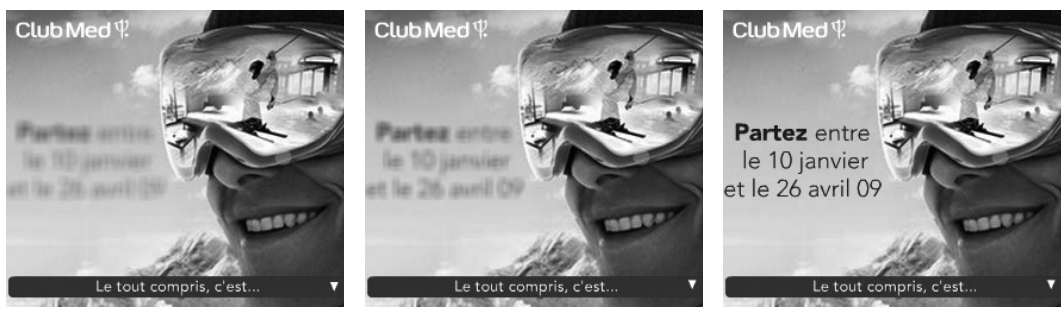

Consultable à l'adresse : < http://www.journaldunet.com/ebusiness/rubriques/ creations-pub-selections-mensuelles/080527-selections-pub-mai/1.shtml > (consulté le 15 juin 2012). L'énoncé « Partez entre le 10 janvier et le 26 avril 09 », d'abord flou, apparaît progressivement, se stabilise pendant quelques instants avant de redevenir flou.

L'annonce commerciale suivante a été repérée sur le site des parfums $K{ }^{4}{ }^{42}$. N'étant plus visible actuellement (août 2013), elle s'étalait pendant plusieurs années sur une grande partie de la page-écran. Le fond de la page-écran était constitué d'une image évanescente qui renvoyait au dernier parfum de la marque, et pouvait donc changer au fil des saisons.

42. [En ligne] : < http://www.kenzoparfums.com/ >. Consulté le $1^{\text {er }}$ février 2010, il n'est plus visible sous cette forme actuellement. 
Figure 19. Capture d'écran d'un extrait du site Kenzo parfums qui n'est plus visible à l'heure actuelle (2013)

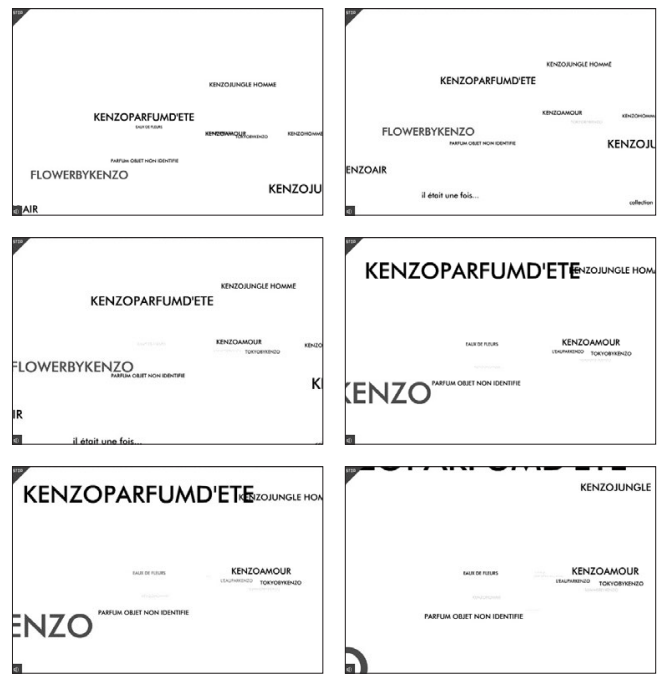

Consultable à l'adresse : < http://www.alexandrasaemmer.fr/corpus/ trajectoire/kenzo.mov >. Les noms de parfums changent progressivement de taille, donnant l'impression de s'approcher du lecteur.

Je décris l'animation telle qu'elle se présente sur la capture d'écran que j'ai mise en ligne ${ }^{43}$. Dans un rectangle blanc s'approchent des noms de parfums, par changement de taille progressif, jusqu'à passer hors champ. La couleur des polices ne perd jamais en intensité lors de cette traversée. Une fois disparus vers le hors-champ, les mêmes noms de parfums resurgissent du centre de l'espace de lecture. Les noms s'affichent en noir, à part quelques exceptions : Kenzopower apparaît en violet sombre, Flower by Kenzo en rose fuchsia. Le mouvement des mots est accompagné d'un son : des bruits de pas s'approchent sur un fond sonore qui pourrait être celui d'une ville, ou alors celui des vagues de la mer. Une voix de femme suave énonce " Il était une fois... Kenzo », suivi d'un petit rire. Les pas s'éloignent. Reste un léger bruitage de fond accompagné d'une musique lente, douce, et d'un court morceau de chant. L'animation tourne

43. [En ligne] : < http://www.alexandrasaemmer.fr/corpus/trajectoire/kenzo.mov >. 
en boucle. Un effleurement des mots par le curseur change légèrement l'orientation du mouvement. L'activation d'un nom de parfum mène à un micro-site dédié à celui-ci.

Figure 20. Bannière publicitaire publiée dans une collection de journaldunet
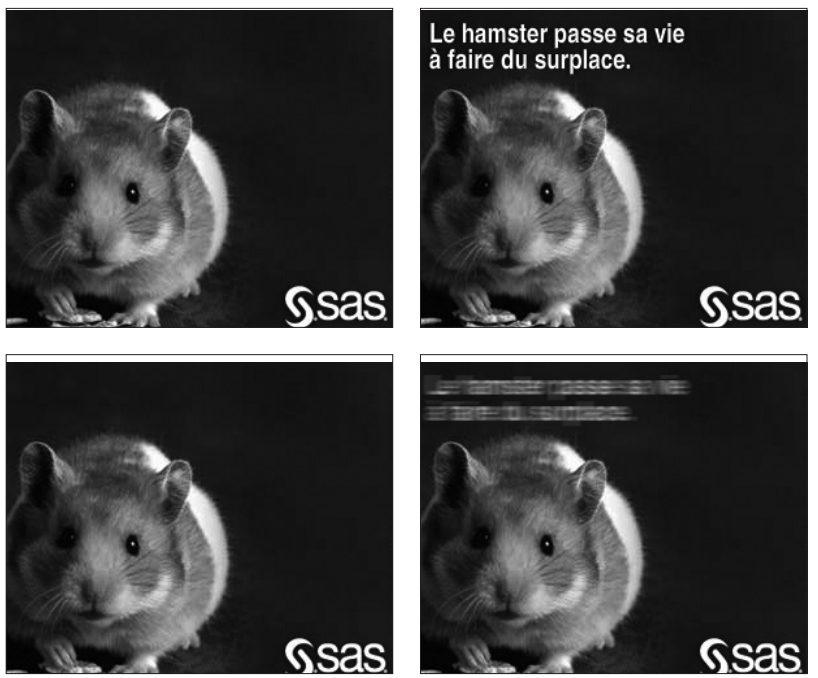

Consultable à l'adresse : < http://www.journaldunet.com/ebusiness/rubriques/ creations-pub-selections-mensuelles/081110-selections-pub-octobre/3. shtml > (consulté le 15 juin 2012). L'énoncé « Le hamster passe sa vie à faire du surplace » apparaît et disparaît à plusieurs reprises avant de se stabiliser.

Le dernier exemple provient de la collection d'octobre 2008 sur le site journaldunet ${ }^{44}$. La bannière faisait la promotion pour SAS Services, un éditeur de solutions informatiques, sur des sites comme decideur, indexa et journaldunet. Le nom de l'agence n'est pas précisé. S'affiche sur un fond sombre, légèrement décalée à gauche, l'image d'un hamster photographié de face. L'une de ses pattes est posée sur de petits débris jaunes. $\mathrm{Au}$-dessus du hamster apparaît et disparaît à plusieurs reprises l'énoncé

44. [En ligne] : < http://www.journaldunet.com/ebusiness/rubriques/creations-pub-selectionsmensuelles/081110-selections-pub-octobre/3.shtml >. 
"Le hamster passe sa vie à faire du surplace », avant de laisser place à la question «Et vous ? " animée par le même mouvement. Dans une banderole marron clignote enfin lentement et à plusieurs reprises l'énoncé "Cliquez pour le savoir ».

\section{Formes-modèles du texte animé}

Dans les cinq bannières publicitaires et annonces commerciales du corpus, le couplage entre textes et mouvements anticipe sur des pratiques de lecture. Lorsque le lecteur s'engage effectivement dans la lecture des textes, un processus que j'ai appelé irradiation iconique se met en place. Les mouvements agissent pourtant potentiellement sur le lecteur même s'il n'entreprend pas cette lecture-déchiffrage.

\section{Formes-modèles « pro-référentielles »}

Comme je l'ai expliqué plus haut, je pars de l'idée que la reconnaissance de certains enchaînements de mouvements est fondée sur des processus d'intégration et de stabilisation d'expériences antérieures : ils forment des unités sémiotiques temporelles que le lecteur reconnaît parce qu'il les a déjà expérimentées ailleurs, par exemple en observant le clignotement d'un bouton d'alarme. Voilà pourquoi j'ai proposé au chapitre précédent de parler de formes-modèles " pro-référentielles ».

\section{« Obsessionnel »}

Les deux premiers exemples du corpus sont marqués par des mouvements de pulsation rapide. Dans l'annonce commerciale de Casino Glamour, le liséré blanc autour de l'énoncé « 50 \$ offerts » apparaît et disparaît de façon rapide et réitérée. Dans la bannière " Les Urbanophiles ", les conseils sont animés par un mouvement de vibration rapide et réitéré. Malgré les différences visuelles entre un mouvement d'apparition-disparition et un mouvement de vibration, les deux enchaînements de mouvements se caractérisent par le même rythme rapide, et renvoient donc à l'unité « obsessionnel ».

Le lecteur peut reconnaître l'obsessionnel parce qu'il a déjà vu clignoter ou vibrer des éléments dans son entourage : j'ai cité l'exemple des boutons d'alarme. Dans le domaine sonore, ce peut être le bip insistant 
d'un réveil, ou une même note de piano répétée rapidement qui se caractérisent par l'obsessionnel ${ }^{45}$. Le potentiel d'action de cette forme-modèle peut donc renvoyer à des idées de stress, de nervosité, d'urgence et d'excitation, ou disons plutôt : il peut créer de telles émotions et sensations chez le lecteur, même si celui-ci ne lit pas le texte associé au mouvement.

\section{« Trajectoire inexorable / à but défini »}

L’unité sémiotique temporelle, appelée " trajectoire inexorable ", est une unité à phase unique, présentant une évolution linéaire qui se caractérise par une "prévisibilité de la non-fin ". Dans le domaine musical, elle est par exemple représentée par un son qui n'en finit pas de monter ${ }^{46}$.

Dans la bannière "Les Urbanophiles », les conseils ne sont pas seulement animés par un mouvement de vibration rapide, mais bougent de gauche à droite vers le hors-champ, sans que la couleur des polices de caractères ni les vibrations perdent en intensité. Dans l'annonce commerciale Parfums Kenzo, un tel mouvement continu anime les noms de parfums, sauf qu'ils surgissent ici du fond vers le hors-champ. Malgré certaines différences visuelles, les deux mouvements peuvent s'apparenter à une trajectoire inexorable grâce au caractère continu et indéfini du mouvement mobilisé.

Le lecteur reconnaît potentiellement une trajectoire inexorable parce qu'il a déjà vu des choses ou des personnes décrire un tel mouvement continu. L'icône pourrait donc renvoyer à des idées de transformation ou de dynamisme constants sans perte de matière et d'énergie. Un tel mouvement rassure potentiellement le lecteur sur sa continuité sans limites.

Le mouvement mobilisé dans la bannière du Club Med se distingue des trajectoires inexorables sur un point important. Les injonctions comme «Partez entre le 10 janvier et le 26 avril 09 », d'abord floues, deviennent progressivement lisibles : ce mouvement s'apparente à une trajectoire, sauf qu'elle s'arrête à son point culminant. Dans le projet de recherche "Signes et figures ${ }^{47}$, nous avons décidé d'appeler cette unité une " trajectoire à but défini ». Elle pourrait renvoyer à des idées de matérialisation, de gain, de révélation et de stabilisation.

45. « Obsessionnel », [En ligne] : < http://www.labo-mim.org/site/index.php?2008/08/22/36-obsessionnel >.

46. « Trajectoire inexorable », [En ligne] : < http://www.labo-mim.org/site/index.php?2008/08/22/28trajectoire-inexorable $>$.

47. Projet «Signes et figures de la création numérique », initié en 2009, avec Philippe Bootz, Serge Bouchardon, Jean Clément et Alexandra Saemmer. Ouvrage en cours de préparation. 


\section{«Sur l'erre »}

Dans la bannière Club Med, les énoncés « Partez entre le 10 janvier et le 26 avril 09 » et " 200 euros offerts à deux* » se stabilisent pour quelques instants. Ensuite ils redeviennent flous, et s'effacent progressivement. Ce mouvement pourrait constituer un correspondant visuel de l'unité sémiotique temporelle " sur l'erre ». Cette unité est délimitée dans le temps et se caractérise par une extinction progressive par disparition naturelle de l'énergie ${ }^{48}$, comme une baisse continue du volume sonore jusqu'au silence. Rappelant au lecteur des êtres ou des choses qui ont disparu de son entourage, le potentiel d'action du « sur l'erre » peut renvoyer à des idées de perte et de disparition inexorables. Le lecteur éprouve potentiellement un sentiment de tristesse ou de frustration en percevant leur extinction progressive.

\section{«Stationnaire »}

Alors que l'obsessionnel peut créer des sensations de nervosité, d'excitation, d'urgence et de stress, et alors qu'une frustration pourrait résulter de l'idée de perte progressive associée au « sur l'erre », l'unité « stationnaire » se caractérise par le paradoxe du « mouvement sur place ». Même si elle peut comprendre des éléments aléatoires ou pseudoaléatoires, en répétition plus ou moins variée, ceux-ci ne perturbent pas la stabilité générale $d u$ mouvement ${ }^{49}$. Dans le domaine sonore, le stationnaire peut par exemple correspondre à une note de flûte maintenue longtemps avec de petites variations d'intensité.

Les mouvements visuels du texte dans la bannière pour SAS Services s'apparentent à cette unité sémiotique temporelle. Plusieurs énoncés se stabilisent progressivement sur le fond de la bannière, après avoir été parcourus par des mouvements d'apparition et de disparition transitoires. $\mathrm{Au}$ niveau visuel, la première partie de ce " stationnaire » peut certes faire penser à un " obsessionnel ». Le clignotement se résorbe pourtant vite dans une stabilité globale. Le potentiel d'action renvoie ainsi à des idées de continuité, voire de monotonie, malgré une certaine vitalité et un dynamisme intérieurs. L'attention du lecteur est potentiellement attirée par ces mouvements transitoires, qui se résorbent si rapidement qu'il n'a

48. «Sur l'erre », [En ligne] : < http://www.labo-mim.org/site/index.php?2008/08/22/45-sur-l-erre >.

49. Exemples de "stationnaires » du corpus consultables en capture vidéo : < http://www.alexandrasaemmer.fr/corpus/stationnaire/ >. 
peut-être pas le temps de les capter consciemment. Ils pourraient donc anticiper à la fois sur des sensations d'excitation et de frustration.

Le tableau ci-dessous liste encore une fois les formes-modèles proréférentielles du texte animé identifiées dans le corpus.

\section{Tableau 14. Formes-modèles pro-référentielles du texte animé}

\section{Trajectoire (inexorable} ou à but défini)

unité non délimitée ou limitée dans le temps, à phase unique, avec une évolution linéaire.
Apparition progressive de l'(infiniment) grand vers le petit ; $\mathrm{du}$ petit vers l'(infiniment) grand. Agrandissement progressif, rétrécissement progressif. Changement de position à vitesse égale.

Disparition progressive ; floutage, décomposition ; changement de position par perte d'énergie.

unité délimitée dans le temps, à une seule phase. Extinction progressive par disparition d'énergie.

Obsessionnel

unité non délimitée dans le temps, à phase unique, constituée d'une formule dont la répétition crée une pulsation.

\section{Stationnaire}

unité délimitée dans le temps, à phase unique, à progression non linéaire, constituée de deux profils successifs opposés.
Clignotement ; changement de couleur brusque et répété ; changement de position brusque et répété ; agrandissement/ rétrécissement répété.

Apparition/disparition lente et réitérée, changement de taille réitéré mais lent ; changement de couleur lent/peu contrasté et réitéré ; déplacement « surplace ».

En tant que formes-modèles qui clignotent, disparaissent ou traversent l'espace de la page-écran, tous ces enchaînements de mouvements peuvent agir sur le lecteur même s'il ne déchiffre pas les textes couplés à eux. Couplés à des textes, ils irradient celui-ci de diverses manières, formant des procédés rhétoriques. 
Figures de la lecture du texte animé

Dans le cas de la publicité en ligne, l'auteur d'une animation textuelle ne peut certainement pas compter sur le fait que le lecteur y prête une attention consciente. Couplant des textes à des mouvements, les auteurs des exemples sélectionnés anticipent néanmoins aussi sur des pratiques de lecture attentives.

Figures de la lecture « affirmative »

Toutes les bannières et annonces commerciales ont comme objectif d'attirer l'attention du lecteur. En dehors de cette évidence, certaines animations textuelles mettent à profit le potentiel d'action du mouvement pour renforcer celui du texte. Ils font appel à un lecteur qui se laisse convaincre par le caractère doublement affirmatif du message. Ces couplages sont donc appelés figures de la lecture « affirmative ».

\section{Couplage « emphatique »}

Dans l'annonce commerciale pour Casino Glamour, un liséré blanc apparaît et disparaît de façon rapide et réitérée autour de l'énoncé « 50 \$ offerts ». J'ai rapproché ces mouvements de l'unité sémiotique temporelle " obsessionnel ", qui renvoie potentiellement à des émotions de stress, de nervosité, d'urgence et d'excitation. L'énoncé « 50 \$ offerts » promet un cadeau pécuniaire, anticipant sur un sentiment de convoitise chez le lecteur. Cette sensation est soulignée par la pulsation rapide autour de l'offre : les potentiels d'action du texte et du mouvement se confirment et se renforcent mutuellement sous forme de couplage « emphatique ».

Inséré dans une annonce commerciale pour un Casino en ligne, le mouvement peut par ailleurs rappeler les enseignes clignotantes dans les salles de jeux, et installer le lecteur dans une ambiance électrisée. Juxtaposé aux injonctions « Jouez avec votre argent » et « Jouez maintenant », le couplage préfigure un lecteur prêt à se laisser prendre au jeu de cette double affirmation, textuelle et sensorielle.

\section{Couplage « additionnel »}

Dans la bannière pour l'opérateur Club Med, les énoncés « Partez entre le 10 janvier et le 26 avril 09 » et « 200 euros offerts à deux* (*voir 
conditions sur le site) » se relayent. Chaque énoncé est d'abord affiché de façon floue, puis devient progressivement lisible. Ce mouvement peut être considéré comme un correspondant visuel de l'unité sémiotique temporelle " trajectoire à but défini », qui renvoie potentiellement à des idées de matérialisation progressive, de révélation et de stabilisation. Au bout de quelques instants de stabilisation, l'énoncé redevient pourtant flou - mouvement qui peut être rapproché de l'unité "sur l'erre ", potentiellement associée à des idées de perte et de disparition inexorables.

Le premier énoncé textuel indique que l'offre du Club Med est limitée dans le temps. Le deuxième énoncé renvoie à l'idée d'un cadeau offert en cas de voyage à deux. La combinaison de la " trajectoire à but défini » et du " sur l'erre », couplée à l'énoncé " Partez entre le 10 janvier et le 26 avril 09 », souligne à quel point l'offre est limitée dans le temps : elle est lancée, se stabilise pendant quelques instants - matérialisation rassurante ; puis elle est retirée du champ de vision, anticipant sur les émotions d'un lecteur qui n'aura pas su profiter de l'offre. Je propose d'appeler couplage " additionnel » cette combinaison entre texte et mouvement, où les potentiels d'action non seulement se renforcent autour d'une idée principale, mais où plusieurs idées et sensations s'additionnent.

Dans le cas de la bannière pour le Club Med, la même combinaison entre la " trajectoire à but défini » et le "sur l'erre » se trouve couplée à l'énoncé « 200 euros offerts à deux »- énoncé qui ne reprend pas l'idée de la limitation temporelle de l'offre. Le potentiel d'action de cette relation entre texte et mouvement illustre, de façon plus claire encore, ce que j'entends par un couplage " additionnel » : alors que le texte renvoie à l'idée d'un cadeau, l'unité « sur l'erre » est potentiellement génératrice d'une émotion de perte et de frustration.

Les animations textuelles côtoient des images renvoyant aux joies des vacances au ski. Alors que ces images restent stables, les couplages entre textes et images rappellent le caractère éphémère de ces visions à celui qui ne profite pas de l'offre. La banderole rouge interactive en bas de la bannière permet au lecteur de s'informer sur les conditions du tout compris.

Les procédés rhétoriques mis en œuvre dans les couplages entre textes et mouvements dans leur contexte proposent une sorte de raisonnement qu'il serait possible de paraphraser par un discours argumentatif : " Vous pouvez profiter de l'offre pendant un certain temps : voilà les joies qui vous sont promises ; si vous ne le faites pas, l'offre disparaîtra inexorablement ; néanmoins, avant de vous décider, voilà les conditions de l’offre ». 
Cette bannière anticipe donc sur un lecteur prêt à se laisser convaincre par l'addition entre les potentiels d'action du texte et du mouvement.

Figures de la lecture « immersive »

En créant des ambiances, sensations et émotions déjà expérimentées ailleurs, certains couplages entre texte et mouvements préfigurent un lecteur prêt à s'immerger, au moins momentanément, dans un texte devenu simulacre de référent.

\section{« Ciné-gramme »}

Dans un couplage que j'ai proposé plus haut d'appeler " cinégramme ", l'irradiation iconique est poussée à l'extrême de sorte que se crée un simulacre de référent : le mot semble se transformer au moins partiellement en " chose » lors de la lecture (voir aussi le ciné-gramme « et la *bombe* faisait tic-tac » dans l'hyperfiction Zeit für die Bombe analysé dans la partie "Figures de la lecture et formes-modèles de la pageécran dans le discours narratif », p. 183). Le ciné-gramme anticipe sur des pratiques de lecture immersives, lors desquelles le lecteur suspend son incrédulité face aux pouvoirs de représentation du texte.

Les noms des parfums Kenzo sont animés sur le site de la marque en s'agrandissant progressivement, comme s'ils s'approchaient du lecteur avant de disparaître vers le hors-champ. Comme l'intensité de la couleur des lettres ne faiblit jamais, le lecteur peut supposer que leur passage se poursuit indéfiniment. J'ai donc rapproché ce mouvement de l'unité sémiotique temporelle " trajectoire inexorable », qui renvoie potentiellement à des idées de cohérence et de persistance. Les mots animés sont des noms de parfums, auxquels le lecteur associe potentiellement des idées d'odeur, d'expérience sensuelle, peut-être d'agrément. Le couplage entre les noms de parfums et le mouvement peut d'abord être qualifié d'additionnel, anticipant sur l'idée d'un parfum persistant.

Le mouvement continu des noms surgissant du centre de l'espace de lecture peut par ailleurs faire penser à l'action d'un diffuseur de parfum qui ne s'arrête jamais, formant donc un ciné-gramme. En effleurant les noms de parfums, le lecteur peut orienter ce nuage d'odeurs vers lui ; en activant l'un des noms, il plonge dans l'ambiance associée à un parfum particulier. Les mots prononcés par la voix féminine, "Il était une fois... Kenzo », soutiennent l'invitation à des pratiques de lecture immersives. 
Le son des pas qui s'approchent et s'éloignent forme en revanche un lieu d'indétermination potentiel avec le couplage entre textes et mouvements. S’agit-il de plaider la persistance, ou au contraire le caractère éphémère de l'instant présent ? Ce lieu d’indétermination pourrait au moins partiellement se résoudre grâce à la mobilisation de certains savoirs culturels. Ne dit-on pas qu'un parfum laisse un sillage, qui nous rappelle une personne même si elle est partie ? Ce sillage ne laisse-t-il pas des traces mnésiques qui peuvent nous ramener à l'enfance - le temps des contes de fée, du " il était une fois »? Le pouvoir des parfums Kenzo résiderait dans ce cas non seulement dans leur persistance, mais dans les traces qu'ils laissent dans notre mémoire en nous plongeant, encore et encore, dans une parenthèse enchantée.

La première animation textuelle dans la bannière SAS Services peut également être considérée comme un ciné-gramme, impliquant cette fois-ci l'unité sémiotique "stationnaire ». À côté de l'image du hamster s'affiche l'énoncé "Le hamster passe sa vie à faire du surplace ». Cet énoncé, d'abord fixe, apparaît et disparaît à plusieurs reprises avant de se stabiliser de nouveau. Le "stationnaire » peut renvoyer à des idées de continuité, voire de monotonie, avec néanmoins une certaine vitalité et un dynamisme intérieurs. L'énoncé " Le hamster passe sa vie à faire du surplace » renvoie au hamster domestiqué, qui effectue des actions répétitives comme courir dans une roue. L'idée du mouvement sur place est imitée par les mouvements de pulsation et leur stabilisation : le lecteur n'est pas seulement invité à lire ce que fait le hamster, il est incité à l'éprouver. Sont donc anticipées dans ce couplage des pratiques de lecture immersives, qui plongent le lecteur dans la monotonie de la vie de hamster animée par un dynamisme réel, mais vain.

L'image du hamster statique renvoie au hamster évoqué dans le couplage texte-mouvement. Le deuxième énoncé, qui se rajoute au bout de quelques instants, s'adresse au lecteur en demandant : « Et vous ? ». Il est animé par le même mouvement stationnaire. Ce couplage additif associe potentiellement l'activité du lecteur à l'idée d'un mouvement sur place.

Après cette nouvelle invitation à la lecture immersive est enfin proposée une solution : "Cliquez pour le savoir ». L'énoncé est animé par un « obsessionnel ». Le texte incite à une action interrompant le mouvement sur place : « Cliquez ». 
Figures de la lecture « déviative »

Dans un exemple du corpus, un lieu d'indétermination s'ouvre entre le texte et l'enchaînement de mouvements dans l'animation textuelle : la lecture immersive se trouve à la fois encouragée et minée. Ce couplage rentre donc dans la catégorie des figures de la lecture « déviative ».

\section{« Ciné-trope ironisant »}

Sur la bannière Auvergne Développement, des lettres fissurées clignotent avant de quitter le cadre de lecture vers le hors-champ. L'énoncé «Soyez stressé, c'est bon pour la productivité » s'affiche par-dessus l'image d'une route goudronnée en vibrant rapidement. Cet enchaînement de mouvements " obsessionnels » renvoie potentiellement à des émotions d'urgence et de danger, de stress et de nervosité. L'obsessionnel semble ainsi mettre en scène l'idée de stress évoquée dans le texte, et former avec lui un ciné-gramme. Son passage rapide hors champ, animé par l'unité « trajectoire inexorable », se couple à cette première combinaison entre texte et mouvement et lui additionne des idées de continuité et de dynamisme constant ; il pourrait faire penser aux mouvements de passage rapide observables dans les grandes villes.

Pourtant, l'injonction " soyez stressé, c'est bon pour la productivité » ne fait pas seulement référence au stress, mais à la " productivité », et associe ces deux idées de façon positive. Il suffirait peut-être de remplacer le contexte iconographique actuel par l'image d'une équipe de cadres discutant d'un projet excitant pour que le couplage entre texte, mouvements et image satisfasse les attentes. Couplé à l'image de la route goudronnée, l'énoncé animé met plutôt au défi ces attentes, déclenchant potentiellement un processus lors duquel le lecteur projette sur le texte sa propre négation. J'appelle ce couplage un " ciné-trope ironisant ». L'oscillation possible entre un sens littéral, exacerbé ici par la forme-modèle animée, et le renversement ironique préfigure des pratiques qui varient entre immersion et mise à distance.

Dans les trois autres conseils qui s'enchaînent dans la bannière, le potentiel d'action du couplage entre texte et mouvement est plutôt de l'ordre additionnel. La recommandation potentiellement sarcastique « Dites bonjour à votre patron, c'est lui qui vous paye » se trouve non seulement soulignée par les mouvements : des idées de nervosité, de stress, d'urgence et de passage inexorable s'y trouvent également associées. Il en 
est de même pour l'énoncé « Portez un costume gris, vous serez assorti à la couleur du ciel ».

Un séjour en Auvergne est proposé comme solution à la situation de stress inexorable. Toutes les recommandations sont présentées sous forme de conseils numérotés - clin d'œil aux célèbres affiches publicitaires de la marque de lingerie féminine Aubade ${ }^{50}$.

Si le lecteur préfiguré par cette bannière oscille entre immersion et distance critique, les mouvements seuls créent potentiellement une ambiance de stress et d'excitation - ambiance que cette bannière dénonce en l'exacerbant, un peu comme si elle dénonçait sa propre stratégie de communication.

Le tableau ci-dessous liste encore une fois les figures de la lecture du texte animé et leurs procédés.

\section{Tableau 15. Figures de la lecture du texte animé et leurs procédés}

Figures de la lecture affirmative

\begin{tabular}{|c|c|}
\hline $\begin{array}{l}\text { Couplage emphatique } \\
\text { préfigure un lecteur prêt à se } \\
\text { laisser convaincre par la double } \\
\text { affirmation, textuelle et sensuelle. }\end{array}$ & $\begin{array}{l}\text { Le potentiel d'action du texte et du } \\
\text { mouvement se confirme. L'irradiation } \\
\text { iconique souligne et renforce le } \\
\text { potentiel d'action du texte. }\end{array}$ \\
\hline $\begin{array}{l}\text { Couplage additionnel } \\
\text { préfigure un lecteur prêt à se laisser } \\
\text { convaincre par l'addition entre les } \\
\text { potentiels d'action du texte et du } \\
\text { mouvement, qui éventuellement } \\
\text { propose un raisonnement. }\end{array}$ & $\begin{array}{l}\text { Combinaison entre le texte et des } \\
\text { formes-modèles pro-référentielles, où } \\
\text { les potentiels d'action non seulement se } \\
\text { renforcent autour d'une idée principale, } \\
\text { mais où plusieurs idées s'additionnent } \\
\text { et complexifient l'énoncé. }\end{array}$ \\
\hline
\end{tabular}

Figures de la lecture immersive

Ciné-gramme
préfigure un lecteur qui suspend
au moins transitoirement son
incrédulité face aux pouvoirs de
représentation du langage.

L'irradiation iconique est poussée à l'extrême de sorte que se crée un simulacre de référent : le mot semble se transformer au moins partiellement en « chose » lors du processus de lecture.

50. « Leçons de séduction » d'Aubade, [En ligne] : < http://aubade.com/Le\%C3\%A7ons\%C2\%A0de\% C2\%A0S\%C3\%A9duction >. 


\section{Figures de la lecture déviative}

\begin{tabular}{|l|l|}
$\begin{array}{l}\text { Ciné-trope ironisant } \\
\text { préfigure un lecteur qui oscille } \\
\text { entre immersion et renversement } \\
\text { critique de l'énoncé. }\end{array}$ & $\begin{array}{l}\text { Le couplage entre texte et forme- } \\
\text { modèle pro-référentielle met } \\
\text { potentiellement au défi les attentes } \\
\text { du lecteur, déclenchant un processus } \\
\text { lors duquel le lecteur projette sur } \\
\text { le texte sa propre négation. }\end{array}$
\end{tabular}




\section{CORPUS D'ARTICLES JOURNALISTIQUES DU CHAPITRE III

Ces articles sont liés à la partie « Figures de la lecture du texte numérique dans les discours informatif et argumentatif ».

1. « Pourquoi la France déroule le tapis rouge pour Hu Jintao », Lexpress.fr, 4 novembre 2010. [En ligne] : < http://www.lexpress.fr/actualite/economie/pourquoi-la-france-deroule-le-tapis-rouge-pour-hu-jintao_933726. html >.

2.Andriamanana Tefy, « Logement : Delanoë creuse-t-il la fracture sociale ? », Marianne2.fr, 29 décembre 2010. [En ligne] : < http:// www.marianne2.fr/Logement-Delanoe-creuse-t-il-la-fracture-sociale_ a201109.html >.

3. Fanette, "Comment Nicolas Sarkozy se "DSKise” en vue de la présidentielle de 2012 », Lepost.fr, 25 janvier 2011. [En ligne] : < http://www. lepost.fr/article/2011/01/25/2382305_comment-nicolas-sarkozy-sedskise-en-vue-des-presidentielles-de-2012.html >.

4.Tian, « Disparition de Laëtitia : François Hollande demande qu'on arrête “de faire des annonces de circonstance” », Lepost.fr, 25 janvier 2011. [En ligne] : < http://www.lepost.fr/article/2011/01/25/2383098_disparition-de-laetitia-francois-hollande-demande-qu-on-arrete-de-fairedes-annonces-de-circonstance.html >.

5. Saulnier Julie, « Récidive : une "proposition de loi Pornic” ?», Lexpress. fr, 26 janvier 2011. [En ligne] : < http://www.lexpress.fr/actualite/societe/ recidive-une-proposition-de-loi-pornic_955890.html >. 
6. Pinsolle Laurent, "Euthanasie : donner aux malades le choix », Marianne2.fr, 27 janvier 2011. [En ligne] : < http://www.marianne2.fr/ Euthanasie-donner-aux-malades-le-choix_a202135.html >.

7. «Laëtitia : les magistrats dénoncent “l'amalgame” », Lexpress.fr, 3 février 2011. [En ligne] : < http://www.lexpress.fr/actualite/societe/laetitia-lesmagistrats-denoncent-l-amalgame_958396.html >.

8. Koch François, « Comment l'affaire Laëtitia est devenue politique », Lexpress.fr, $1^{\text {er }}$ février 2011. [En ligne] : < http://www.lexpress.fr/ actualite/societe/justice/comment-l-affaire-laetitia-est-devenue-politique_957534.html >.

9. Andrieu Gérald, "Un spécialiste du "care" au chevet de la pensée de Nicolas Hulot », Marianne2.fr, 7 février 2011. [En ligne] : < http:// www.marianne2.fr/Un-specialiste-du-care-au-chevet-de-la-pensee-deNicolas-Hulot_a202450.html >.

10.Vincent Olivier, « Bioéthique : comment les parlementaires veulent faire évoluer la loi », Lexpress.fr, 8 février 2011. [En ligne] : < http:// www.lexpress.fr/actualite/sciences/sante/bioethique-comment-la-loipourrait-evoluer_959762.html >.

11. Brunel Charlotte, "Acne, la marque suédoise qui monte ", Lexpress.fr, 18 février 2011. [En ligne] : < http://www.lexpress.fr/styles/mode/acnela-marque-suedoise-qui-monte_963465.html >.

12. "Nicolas Sarkozy dans "Parole de Français" : comment sont choisis les participants ? », Lepost.fr, 3 février 2011. [En ligne] : < http://www. lepost.fr/article/2011/02/03/2393850_nicolas-sarkozy-dans-parole-defrancais-comment-sont-choisis-les-participants.html >.

13. Andriamanana Tefy, « Pornic : une mauvaise coopération police/gendarmerie ? », Marianne2.fr, 20 février 2011. [En ligne] : < http://www. marianne2.fr/Pornic-une-mauvaise-cooperation-police-gendarmerie_ a202924.html >. 
14. Veron Michel, "Sarkozy rate son pari sur l'international », Lexpress. fr, 23 février 2011. [En ligne] : < http://www.lexpress.fr/actualite/politique/sarkozy-rate-son-pari-sur-l-international_965548.html > .

15.Veron Michel, "Qu'a rapporté la visite de Kadhafi à la France ? ", Lexpress.fr, 22 février 2011. [En ligne] : < http://www.lexpress.fr/actualite/politique/qu-a-rapporte-la-visite-de-kadhafi-a-la-france_965242. html >.

16. Pierre-Alain, « Libération d'une otage française d'AQMI : contre une rançon ? », Lepost.fr, 26 février 2011. [En ligne] : < http://www.lepost. fr/article/2011/02/26/2418070_otages-enleves-au-niger-francoise-larribe-a-ete-liberee-contre-une-rancon.html >.

17. Andriamanana Tefy, « L'insécurité et la violence progressent dans les hôpitaux », Marianne2.fr, 25 février 2011. [En ligne] : < http://www. marianne2.fr/L-insecurite-et-la-violence-progressent-dans-les-hopitaux_a203140.html >.

18. Bousquet Élodie, "Livre numérique : quelle bibliothèque pour demain ? », Lexpress.fr, 26 février 2011. [En ligne] : < http://www. lexpress.fr/culture/livre/livre-numerique-quelle-bibliotheque-pourdemain_966337.html >.

19. Dupont Laureline, "Fillon enfile le costume de chef du service aprèsvente », Marianne2.fr, 28 février 2011. [En ligne] : < http://www. marianne2.fr/Fillon-enfile-le-costume-de-chef-du-service-apresvente_a203352.html >.

20. « Que retiendrez-vous du remaniement ? », Lexpress.fr, 28 février 2011. [En ligne] : < http://www.lexpress.fr/actualite/politique/que-retiendrez-vous-du-remaniement_966904.html >.

21. « Dior peut-il licencier Galliano pour des propos racistes ? », Lexpress. fr, $1^{\text {er }}$ mars 2011. [En ligne] : < http://www.lexpress.fr/emploi-carriere/emploi/dior-peut-il-licencier-galliano-pour-des-proposracistes_967671.html >. 
22. Andriamanana Tefy, « Face aux crises migratoires, Bruxelles est dans les choux », Marianne2.fr, 2 mars 2011. [En ligne] : <http://www. marianne2.fr/Face-aux-crises-migratoires-Bruxelles-est-dans-leschoux_a203368.html>.

23. Veron Michel, « Laïcité : "La République est hypocrite” », Lexpress.fr, 4 mars 2011. [En ligne] : <http://www.lexpress.fr/actualite/politique/ laicite-la-republique-est-hypocrite_968875.html>.

24.Veron Michel, "Le candidat Sarkozy enrôle l'histoire de France ", Lexpress.fr, 3 mars 2011. [En ligne] : <http://www.lexpress.fr/actualite/ politique/le-candidat-sarkozy-enrole-l-histoire-de-france_968473. html>.

25. Lemarié Alex, «Progression du FN : पL'UMP et le PS ont chacun une part de responsabilité" », Lepost.fr, 9 mars 2011, < http://www.lepost. fr/article/2011/03/08/2427830_progression-du-fn-l-ump-et-le-ps-ontchacun-une-part-de-responsabilite.html >. 


\section{CONCLUSION. SYNTHESE ET PERSPECTIVES}




\section{CONCLUSION. SYNTHĖSE ET PERSPECTIVE}

$\mathrm{L}$ a rhétorique, art de persuader et de convaincre, stratégie de la délibération et de la discussion, art poétique et méthode de décryptage critique des discours, est fondée sur des figures qui proposent une restructuration du réel adressée à un public. La rhétorique du texte numérique est une méthodologie au carrefour des méthodes : à la fois une science du texte en tant qu'elle étudie celui-ci comme matière potentiellement signifiante, et une science de ses lectures en tant qu'elle se penche sur les pratiques individuelles et socialement partagées du sens.

Depuis ses origines antiques, la rhétorique est considérée comme une approche à la fois théorique et pratique qui s'intéresse aux contenus, contextes matériels, et aux publics potentiels et réels d'un discours. Elle se décline traditionnellement en cinq composantes : l'inventio, la dispositio, l'elocutio, l'actio et la memoria.

Le texte numérique est caractérisé par de nouveaux procédés rhétoriques, fondés en outre sur l'hyperlien et le mouvement, qu'il s'agissait d'identifier dans ce livre. Tout hyperlien est d'abord un texte manipulable qui implique le corps du lecteur : les gestes effectués par le lecteur sur le texte à lire influencent la réception de celui-ci. J'ai proposé d'appeler unités sémiotiques de la manipulation certains enchaînements de gestes, qui renvoient à un référent d'expérience dans l'entourage du lecteur : les gestes d'appui et de relâchement sur un hyperlien, par exemple, peuvent faire penser à l'activation d'un interrupteur. Les couplages entre textes et unités sémiotiques de la manipulation forment des procédés rhétoriques qui relèvent de l'élocution.

Il en est de même pour les procédés rhétoriques du texte animé, qui sont fondés sur un couplage entre le texte à lire et des mouvements. Là aussi, certains enchaînements de mouvements peuvent faire penser à des référents d'expérience : le clignotement peut ainsi rappeler des éléments clignotants dans l'espace urbain. J’ai proposé d'appeler unités sémiotiques temporelles ces enchaînements de mouvements, en référence à des unités identifiées dans le domaine sonore qui semblent partager des caractéristiques fondamentales avec certains mouvements visuels (le tic-tac 
insistant d'une bombe et le clignotement font ainsi partie d'une même unité appelée « obsessionnel »).

Les unités sémiotiques temporelles et les unités sémiotiques de la manipulation font partie des formes-modèles [Jeanneret, Souchier, 2005] du texte numérique. D’autres formes-modèles du texte, déjà bien connues dans le domaine du texte papier, sont la couleur, la taille et la police de caractère. Tout texte numérique s'inscrit enfin dans le cadre d'une pageécran, qui est à son tour caractérisée par un certain nombre de formesmodèles, par exemple la couleur du fond de la page.

La rhétorique du texte numérique considère le processus de lecture comme une rencontre, toujours forcément partielle, entre l'anticipation de pratiques par les procédés rhétoriques, et les attentes individuelles et socialement partagées du lecteur. Il s'agit donc, d'une part, de prendre en compte les imaginaires du dispositif et de la lecture numériques, des médias et des genres textuels, en s'appuyant sur des études empiriques (questionnaires, entretiens, observations et analyses de discours théoriques). D'autre part, il s'agit d'analyser comment le texte numérique à lire, à regarder et à manipuler, cadré dans une page-écran et ancré dans son dispositif, anticipe sur les pratiques de lecture et les constitue. Ces modélisations de pratiques par le texte numérique sont appelées figures de la lecture.

Au chapitre II, j'ai d'abord étudié un grand nombre de discours consacrés au texte et à la lecture numériques. Je me suis également penchée sur des études empiriques consacrées à la lecture et aux pratiques des dispositifs numériques. J'ai constaté que l'idée d'un hyperlien dialogique, défini comme médiateur entre idées divergentes, et celle d'un hyperlien informationnel, défini comme lien stable entre des données factuelles, constituent des imaginaires récurrents.

Après le parcours dans l'horizon d'attente du lecteur de textes numériques, j'ai débuté l'étude des corpus journalistique, littéraire et publicitaire par l'identification des formes-modèles du texte et de la page-écran. En cohérence avec la présomption d'information qui prime chez beaucoup de lecteurs face au texte numérique, en cohérence aussi avec la rapidité considérée par beaucoup comme caractéristique de la lecture numérique, les formes-modèles pro-extensives préfigurent un lecteur impatient qui souhaite aller vite : prenons l'exemple de la police de caractère sans empattements adoptée par beaucoup de journaux en ligne, qui est censée favoriser la lecture rapide à l'écran. 
Les formes-modèles pro-intensives, en revanche, font appel à un lecteur prêt à prendre le temps d'une exploration lente et concentrée. Certains journaux en ligne ont adopté une police de caractère avec empattements pour favoriser cette pratique.

Les formes-modèles pro-interventives anticipent sur un lecteur qui ne se contente plus de lire, mais qui désire laisser lui-même des traces d'existence sur la page-écran : ne serait-ce qu'en apposant un "like». La figuration de ce lecteur-auteur hyperactif s'est trouvée renforcée par l'émergence du Web 2.0.

Les formes-modèles pro-référentielles préfigurent un lecteur prêt à s'abandonner aux simulacres de référent mobilisés par la couleur, les unités sémiotiques temporelles ou les unités sémiotiques de la manipulation, qui renvoient par leurs caractéristiques sensibles à des référents d'expérience : prenons l'exemple du mot « cœur » rouge qui clignote dans le récit d'une histoire d'amour, et qui renverrait à la fois à la couleur et au rythme de l'organe désigné.

Les formes-modèles non référentielles anticipent sur un lecteur prêt à considérer les formes et les couleurs du texte et de la page-écran comme des entités abstraites : la couleur, par exemple, ne renvoie dans ce cas plus à un référent d'expérience, mais à sa propre plasticité.

Les formes-modèles pro-depréhensives, enfin, préfigurent un lecteur qui accepte de perdre prise sur l'interface : le clic sur le bouton de fermeture d'une fenêtre pop-up provoque dans ce cas l'invasion d'une multiplicité d'autres fenêtres pop-up. Ces formes-modèles potentiellement surprenantes ou déroutantes semblent plus facilement acceptables dans des textes relevant des genres littéraires, que dans le journalisme.

Tableau 16. Formes-modèles du texte et de la page-écran

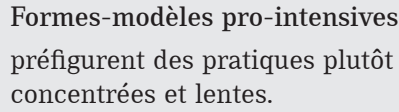

Polices de caractère avec empattements ; relative longueur des articles et paragraphes ; structuration des articles rappelant parfois un pendant papier. 


\begin{tabular}{|c|c|}
\hline $\begin{array}{l}\text { Formes-modèles pro-extensives } \\
\text { préfigurent un lecteur pressé. }\end{array}$ & $\begin{array}{l}\text { Structuration des articles mettant en } \\
\text { évidence les informations clé du texte ; } \\
\text { encadrés synthétiques hyperliés ; police } \\
\text { sans empattements ; haut niveau de } \\
\text { tabularité de la page-écran ; marquage } \\
\text { des hyperliens incitant à la navigation. }\end{array}$ \\
\hline $\begin{array}{l}\text { Formes-modèles pro-interventives } \\
\text { préfigurent un lecteur souhaitant } \\
\text { laisser des traces dans le texte. }\end{array}$ & $\begin{array}{l}\text { Espace « commentaires » et autres } \\
\text { invitations à la participation active } \\
\text { du lecteur, possibilité d’accéder au } \\
\text { backoffice renvoyant à des formats } \\
\text { numériques modifiables comme } \\
\text { le blog ; symboles invitant au } \\
\text { partage sur les réseaux sociaux. }\end{array}$ \\
\hline $\begin{array}{l}\text { Formes-modèles pro-depréhensives } \\
\text { préfigurent un lecteur acceptant } \\
\text { de perdre prise sur l'interface. }\end{array}$ & $\begin{array}{l}\text { Pages-écran défilant automatiquement } \\
\text { sans que le lecteur puisse intervenir } \\
\text { sur le rythme ou arrêter le processus ; } \\
\text { multiplication intempestive de } \\
\text { fenêtres pop-up sur la page-écran ; } \\
\text { absence de réactivité de certains } \\
\text { éléments d'apparence manipulables. }\end{array}$ \\
\hline $\begin{array}{l}\text { Formes-modèles pro-référentielles } \\
\text { préfigurent un lecteur } \\
\text { prêt à s'abandonner à un } \\
\text { simulacre de référent. }\end{array}$ & $\begin{array}{l}\text { La couleur, la police de caractère, } \\
\text { la forme ou l'étendue de certains } \\
\text { espaces sur la page-écran, les unités } \\
\text { sémiotiques de la manipulation et } \\
\text { les unités sémiotiques temporelles } \\
\text { renvoient potentiellement à } \\
\text { un référent d'expérience. }\end{array}$ \\
\hline $\begin{array}{l}\text { Formes-modèles non référentielles } \\
\text { préfigurent un lecteur prêt à } \\
\text { considérer formes et couleurs } \\
\text { comme des entités abstraites. }\end{array}$ & $\begin{array}{l}\text { La couleur, la police de caractère, la } \\
\text { forme et l'étendue de certains espaces } \\
\text { ne se laissent pas enfermer dans } \\
\text { un signe iconique, mais renvoient } \\
\text { plutôt à leur propre plasticité. }\end{array}$ \\
\hline
\end{tabular}

Les couplages entre les textes à lire et leurs formes-modèles relèvent donc des procédés d'élocution du texte numérique. Des formes-modèles pro-référentielles comme le clignotement peuvent ainsi irradier le texte de sorte que le message s'en trouve affirmé. Fait partie de ces figures de la lecture affirmative le couplage emphatique : par exemple, l'urgence déjà suggérée par le mot " soldes » dans une bannière publicitaire, est renforcé par le clignotement du mot. Le couplage additionnel est également une figure de la lecture affirmative : dans une bannière publicitaire, le texte « 200 euros offerts à deux » est d'abord couplé à un mouvement d'apparition lente (unité " trajectoire »), puis il est suivi d'un mouvement de disparition (" sur l'erre »). Alors que le texte renvoie à l'idée d'un 
cadeau, l'unité « sur l'erre » est potentiellement génératrice d'une émotion de perte et de frustration; ces potentiels s'additionnent dans la bannière. Les figures de la lecture affirmative ne vont généralement pas à l'encontre des attentes du lecteur.

Il en va autrement des figures de la lecture déviative, qui sont plutôt censées mettre au défi les attentes. Dans une hyperfiction, la phrase «*Le temps explose en mille éclats* » se trouve couplée à un clignotement « obsessionnel ». Ce clignotement n'imite nullement l'explosion. Le cinétrope et le kiné-trope (couplage surprenant entre texte et gestes) jouent ainsi avec les pratiques immersives du texte numérique tout en les mettant en branle.

Le ciné-gramme et le kiné-gramme, en revanche, confortent ces pratiques et font donc partie des figures de la lecture immersive. Le texte s'y trouve transformé en simulacre de référent. Je rappelle l'exemple de l'hyperlien posé sur " appuie sur le petit interrupteur " dans une hyperfiction. L'unité " activer ", renvoyant potentiellement à des objets qui s'activent effectivement par un enchaînement rapide de gestes d'appui et de relâchement, se trouve dans cet énoncé couplé au mot « interrupteur ", un objet qui s'active par les mêmes gestes. Est ainsi suggéré au lecteur qu'il appuie sur la chose "interrupteur », et non pas seulement sur le mot.

Tous ces procédés de l'élocution du texte numérique combinent des signes de nature fort différente (par exemple du texte et du mouvement), et font appel à diverses facultés cognitives, sensations et émotions du lecteur.

Les procédés de la disposition du texte numérique anticipent également de plusieurs manières sur l'horizon d'attente du lecteur, et fondent de nouveau des figures de la lecture. J'ai défini l'hyperlien comme une marque d'interprétation : un Auteur relie deux textes entre eux parce qu'il pense qu'ils doivent être reliés ainsi, pour différentes raisons que la rhétorique du texte numérique essaie de sonder. J'ai appelé texte géniteur le texte contenant l'hyperlien : par exemple un article journalistique qui avance une affirmation. J'ai appelé texte relié le texte connecté à cet hyperlien : par exemple un texte proposant une confirmation ou un démenti de cette affirmation. Il s'agit ainsi d'étudier les niveaux de contiguité logique ou temporelle entre les répertoires du texte géniteur et du texte relié dans leur contexte d'édition.

Les figures de la lecture informationnelle font appel à la présomption d'information avec laquelle beaucoup de lecteurs approchent l'hyperlien dans le texte numérique. Les procédés mobilisés dans cette figure sont plus précisément l'hyperlien définissant, l'hyperlien renvoyant à la source, 
l'hyperlien illustratif, l'hyperlien fournissant des preuves, l'hyperlien d'autorité, et l'hyperlien explicatif.

Alors que beaucoup de lecteurs approchent l'hyperlien avec des attentes informationnelles, certains comptent aussi sur une une juxtaposition de points de vue divergents. Les figures de la lecture dialogique répondent à ces attentes, proposant des procédés comme l'hyperlien déplaçant le focus, l'hyperlien comparatif, l'hyperlien croisant des points de vue, et l'hyperlien réinterprétant.

Les figures de la lecture chronologique anticipent sur un lecteur désireux de pouvoir ordonner différents événements sur un axe temporel. L'hyperlien à potentiel d'action successif, l'hyperlien analeptique, l'hyperlien proleptique et l'hyperlien synchronisant proposent au lecteur différentes possibilités de se déplacer en avant ou en arrière sur l'axe temporel des événements racontés.

Les figures de la lecture topologique sont fondées sur un changement de lieu entre texte géniteur et texte relié : l'hyperlien délocalisant préfigure ainsi un lecteur curieux d'explorer des événements qui se déroulent ailleurs, dans un autre lieu que celui décrit par le texte géniteur.

Il va sans dire que toutes ces figures de la lecture de l'hyperlien ne sont pas réservées à un genre textuel précis, et qu'un même hyperlien peut avoir plusieurs potentiels d'action. Pensons à un article journalistique qui décrit un personnage politique de l'extérieur, alors que le texte relié relate le point de vue interne de ce personnage tout en ramenant le lecteur en arrière sur l'axe temporel (par exemple en donnant accès à un souvenir d'enfance raconté par ce personnage). Les réactions du lecteur face aux figures de la lecture, en revanche, ne seront sans doute pas les mêmes lorsqu'elles se trouvent mobilisées dans un texte journalistique, une hyperfiction ou une bannière publicitaire.

Cette remarque vaut tout particulièrement pour la catégorie des figures de la lecture déviative. Ces figures font appel à un lecteur prêt à s'engager dans des découvertes surprenantes. J’ai déjà évoqué les ciné-tropes et kiné-tropes, qui font partie de ces figures de la lecture déviative au niveau de l'élocution. Au niveau de la disposition, l'hyperlien déformatoire, l'hyperlien métaphorisant, et l'hyperlien ironisant font partie de cette catégorie : pensons à cet article qui affirme que les propos d'une autoentrepreneuse lors d'une émission consacrée au gouvernement Sarkozy ont été très complaisants (" on apprenait par la suite qu'elle était fan de François Fillon »), alors que le texte relié montre que la femme conteste cette affirmation. 
Le tableau ci-dessous liste encore les figures de la lecture du texte manipulable et animé qui ont été identifiées dans les corpus.

Les figures relevant de la disposition sont marquées par une trame claire, les figures de l'élocution dans une trame plus foncée.

Tableau 17. Figures de la lecture informationnelle

\section{Hyperlien définissant}

préfigure un lecteur désireux d'en savoir plus sur des mots et notions.
Hyperlien posé sur des mots rares, des notions complexes; le texte relié propose la définition d'un terme technique, d'un dispositif, d'une institution...

Mise en relation du texte géniteur avec la source qui a permis à l'Auteur de circonscrire les contours d'un événement, d'une personnalité...

Mise en relation de certains éléments du texte géniteur avec des illustrations visuelles.

préfigure un lecteur souhaitant accéder à une confirmation de l'information donnée dans le texte géniteur.

\section{Hyperlien fournissant des preuves}

préfigure un lecteur voulant disposer de preuves pour l'information donnée dans le texte géniteur.

\section{Hyperlien d'autorité} préfigure un lecteur se laissant rassurer par une parole d'expert.

\section{Hyperlien explicatif} peut répondre aux attentes de lecteurs cherchant des compléments d'information.

Le texte relié confirme les affirmations avancées par le texte géniteur par des preuves factuelles.

En renvoyant à des personnalités ou des institutions jouissant d'une position d'autorité, l'Auteur espère doter le texte géniteur d'une crédibilité supplémentaire.

Le texte relié décrit les circonstances d'un événement, l'explique en avançant des données factuelles ; forte contiguïté causale avec le texte géniteur. 
Figures de la lecture dialogique

\section{Hyperlien déplaçant le focus}

préfigure un lecteur désireux d'accéder à un aspect différent de celui exposé dans le texte géniteur.

\section{Hyperlien comparatif}

préfigure un lecteur prêt à effectuer le transfert de caractéristiques entre le texte géniteur et le texte relié.

Hyperlien croisant des points de vue préfigure un lecteur acceptant de mettre en perspective les arguments exposés.

\section{Hyperlien réinterprétant}

préfigure un lecteur suffisamment attentif pour démasquer la réinterprétation, ou alors un lecteur qui se laisse manipuler en passant trop rapidement sur le texte.
Le texte relié déplace potentiellement le focus du texte géniteur en ciblant un aspect particulier de la problématique générale, ou en présentant un point de vue connexe.

Texte géniteur et texte relié proposent une analogie entre deux ou plusieurs événements, personnes ou faits.

Hyperlien faisant potentiellement dialoguer des points de vue divergents entre le texte géniteur et le texte relié.

Un événement, un chiffre ou un fait divers ont été réinterprétés par l'Auteur à partir d'une information source, sans que la divergence de points de vue soit annoncée dans le texte géniteur.

Figures de la lecture chronologique

\section{Hyperlien successif}

préfigure un lecteur désireux de découvrir les différents épisodes d'une histoire dans l'ordre, alignés sur la flèche du temps.

\section{Hyperlien analeptique}

préfigure un lecteur curieux de savoir ce qui s'est passé avant les événements racontés dans le texte géniteur.
Le texte géniteur raconte un événement ; l'activation du texte relié permet de découvrir sa suite chronologique : un peu comme si les événements se déroulaient en direct, sous les yeux du lecteur.

Le texte relié propose un saut en arrière sur la flèche temporelle de l'histoire racontée ; d'éventuelles annonces et justifications du saut temporel, ainsi que l'emplacement de l'hyperlien dans le texte géniteur peuvent préparer le terrain au saut temporel. 


\section{Hyperlien proleptique \\ préfigure un lecteur curieux de savoir ce qui se passera dans l'avenir, un certain temps après les événements racontés dans le texte géniteur.}

Le texte relié propose un saut vers le futur lointain. Des annonces dans le texte géniteur, ainsi que l'emplacement de l'hyperlien préparent éventuellement le terrain à ce saut en avant.

Le texte relié permet de découvrir des événements qui se déroulent parallèlement aux événements racontés dans le texte géniteur, dans un même endroit ou pas, avec les mêmes personnages ou pas.

\section{Figures de la lecture topologique}

\section{Hyperlien délocalisant}

préfigure un lecteur curieux d'explorer des événements qui se déroulent dans un autre lieu que celui décrit par le texte géniteur.
Le texte relié propose un changement de lieu par rapport au texte géniteur. L'annonce éventuelle du changement de lieu dans le texte géniteur peut atténuer le risque qu'un lieu d'indétermination émerge à cause de l'abandon de l'unité de lieu.

Figures de la lecture immersive

\section{Kiné-gramme}

préfigure un lecteur prêt à s'immerger au moins momentanément dans un texte devenu simulacre de référent.
L'irradiation iconique apportée par l'unité sémiotique de la manipulation est poussée à l'extrême, de sorte que se crée un simulacre de référent : le mot semble se transformer au moins partiellement en " chose » lors du processus de lecture.

L'irradiation iconique apportée par l'unité sémiotique temporelle est poussée à l'extrême, de sorte que se crée un simulacre de référent : le mot semble se transformer au moins partiellement en " chose » lors du processus de lecture. 
Figures de la lecture affirmative

\section{Couplage emphatique}

préfigure un lecteur prêt à se laisser convaincre par la double affirmation, textuelle et sensuelle.

\section{Couplage additionnel}

préfigure un lecteur prêt à se laisser convaincre par l'addition entre les potentiels d'action du texte et du mouvement, qui éventuellement propose un raisonnement.
Le potentiel d'action de la formemodèle pro-référentielle du texte (unité sémiotique de la manipulation, unité sémiotique temporelle, couleur...) et les répertoires $d u$ texte se confirment et se renforcent mutuellement. L'irradiation iconique souligne et renforce le potentiel d'action du texte.

Le potentiel d'action de la formemodèle pro-référentielle du texte (unité sémiotique de la manipulation, unité sémiotique temporelle, couleur...) et les répertoires du texte non seulement se renforcent autour d'une idée principale, mais plusieurs idées s'additionnent et complexifient l'énoncé.

Figures de la lecture déviative

\section{Hyperlien déformatoire}

préfigure un lecteur pressé, habitué aux hyperliens informationnels ; ou alors un lecteur complice, partageant avec l'Auteur une volonté de subversion.

\section{Hyperlien ironisant}

préfigure un lecteur complice, prêt à effectuer le renversement ironique de certains termes.

\section{Hyperlien métaphorisant}

préfigure un lecteur prêt à se laisser surprendre par des associations inattendues, et à déployer son imagination.
Le texte relié présente des éléments contredisant les affirmations du texte géniteur, sans que ces contradictions soient explicitement annoncées. La frontière entre manipulation préméditée et volonté de contester des discours officiels n'est pas toujours facile à tracer.

La rétroprojection de certains éléments du texte relié sur le texte géniteur y substitue potentiellement sa propre négation.

L'hyperlien métaphorisant « éclaire » des éléments du texte géniteur et, en même temps, les « éclaire à l'infini ». La relation entre texte géniteur et texte relié ne se laisse que partiellement « épuiser » par un processus d'interprétation. 
Kiné-trope

préfigure un lecteur prêt à accepter l'oscillation entre immersion et distance.

Ciné-trope ironisant préfigure un lecteur qui oscille entre immersion et renversement critique de l’énoncé.
L'irradiation iconique agit sur le texte.

Un décalage persiste néanmoins entre les potentiels d'action du texte et de l'unité sémiotique de la manipulation, et trouble l'immersion.

L'énoncé animé met potentiellement au défi les attentes du lecteur, déclenchant un processus lors duquel le lecteur projette ensuite sur le texte sa propre négation.

L'objectif de la rhétorique présentée dans ce livre est de sensibiliser lecteurs, chercheurs, auteurs et éditeurs aux possibles expressifs du texte numérique, et de leur permettre ainsi de mieux connaître et maîtriser ses procédés d'argumentation, de persuasion, de narration, de métaphorisation et de séduction. J'espère que la connaissance de ce champ des possibles favorisera l'émergence d'une nouvelle culture de l'interprétation, réflexive et critique.

La création textuelle sur support numérique réinvente ses formes et figures non seulement en fonction de nouveaux dispositifs, langages de programmation et logiciels avec leurs contraintes et potentialités, mais aussi en répondant à une demande grandissante du public qui commence à accepter la possibilité d'une lecture numérique, que ce soit dans un cadre professionnel ou personnel, pour se plonger dans une fiction, lire le journal, chercher des informations factuelles ou pour apprendre. L'édition numérique soutient, alimente et oriente cette demande, cherche et propose des textes qui explorent, avec plus ou moins d'audace, le champ des possibles de la textualité numérique.

La rhétorique du texte numérique n'est pas un modèle théorique élaboré à côté du marché de l'édition numérique et de la recherche appliquée en technologies de l'information et de la communication ; elle n'est pas déconnectée de l'enseignement des écritures sur ordinateur. Je propose ici la stabilisation d'une approche théorique que j'ai commencé à élaborer il y a une dizaine d'années, certes en réponse aux besoins du chercheur qui souvent manque encore de mots et de concepts pour décrire et analyser ce qu'il voit, observe et lit sur les supports numériques, mais aussi en réponse aux demandes formulées par mes étudiants, futurs designers numériques, 
publicitaires, webmasters éditoriaux et documentalistes, qui souhaitent savoir écrire et éditer du texte numérique en connaissance de cause.

J'ai constaté dans ma pratique d'enseignement que les outils de la rhétorique présentée ici, loin d'enfermer le texte numérique dans des typologies et des concepts, peuvent favoriser l'expérimentation créative : en montrant comment les potentiels d'un texte et d'un mouvement se couplent au sein d'une animation textuelle, et comment ce couplage anticipe sur les pratiques de lecture, la rhétorique ouvre aussi la voie à d'autres couplages possibles.

Émergent donc trois perspectives qui esquissent des terrains d'application de la rhétorique du texte numérique.

\section{RHÉTORIQUE ET FORMATION À LA CULTURE NUMÉRIQUE}

La première perspective concerne l'intégration de la rhétorique du texte numérique dans les pratiques pédagogiques. Beaucoup de lecteurs vont sur Internet pour consulter des informations ; ils espèrent obtenir des réponses précises, fiables, objectives. À travers l'étude du corpus journalistique, j'ai souhaité montrer que certains textes journalistiques avec hyperliens préfigurent pourtant plutôt un lecteur prêt à mettre en perspective des avis divergents. De telles figures peuvent mettre en branle le désir de certitudes (" je vérifie sur Internet ») ; elles peuvent pourtant aussi former un rempart essentiel contre les vérités définitives, grands récits et autres tentatives de re-mythification dans notre société.

Marlène Loicq [2009, 77] définit l'enjeu principal de la formation aux médias comme " la capacité de prendre conscience du caractère polysémique des messages ainsi que de la dimension "construite" de l'information (et de la réalité) ». La rhétorique du texte numérique propose des outils pour le chercheur, l'apprenant, et pour tout lecteur voulant s'engager dans un acte d'interprétation réflexif et critique, ressort dynamique de l'émergence d'un nouveau rapport aux savoirs. Comme l'affirme Claude Baltz [2005], « trouver l'information ne facilite pas la compréhension »: il faut savoir la lire. Voilà pourquoi, en vue d'une complexification des pratiques de lecture, il était urgent de développer des repères et outils conceptuels pour l'analyse et l'interprétation du texte numérique. 


\section{RHÉTORIQUE ET CRÉATION}

J'ai proposé un relevé des figures de la lecture du texte numérique en étudiant trois corpus. J'ai pourtant également modélisé, à plusieurs reprises, des possibles du texte numérique. En modélisant les déclinaisons d'un couplage entre le mot « soldes » et l'unité sémiotique temporelle « obsessionnel », j'ai eu recours à des combinaisons entre texte et mouvement dans le corpus tout en imaginant d'autres possibilités. Les outils méthodologiques de la rhétorique m’ont aidée à repérer les unités de sens d'un texte animé, que j'ai ensuite pu combiner d'une nouvelle façon. Il m'importe de pointer le rôle que l'expérimentation a joué dans l'élaboration de la rhétorique présentée dans ce livre. La connaissance des potentiels d'action du texte numérique et son analyse au regard de nos horizons d'attente actuels sont un enjeu de recherche et un moteur pour l'innovation - telle est en tout cas l'hypothèse que je souhaite avancer ici.

\section{RHÉTORIQUE ET PLAISIR}

Après ce long parcours dans les procédés rhétoriques du texte numérique je reviens, en guise de conclusion, à la question cruciale du plaisir du texte. Même si la prise de conscience des effets de persuasion dans le texte numérique est un enjeu important, et même si la rhétorique vise à identifier les couches de médiation du texte numérique pour faire émerger une attitude réflexive chez le lecteur, son objectif ne se réduit pas à la proposition d'outils de défense contre les effets de persuasion ou de manipulation du texte.

J'espère avoir démontré qu'il est possible de non seulement confirmer des informations par hyperlien, mais aussi de relier une citation à sa référence ou un terme technique à sa définition, et d'attirer l'attention du lecteur par un clignotement. En effet, toute cette multiplicité d'exemples a été convoquée pour montrer aux chercheurs, lecteurs et auteurs de textes numériques que l'hyperlien peut aussi être mobilisé pour argumenter ; qu'un enchaînement de gestes, couplé à des mots, peut immerger le lecteur dans l'événement raconté ; qu'un enchaînement de mouvements peut provoquer le renversement ironique d'un énoncé, et qu'il est même possible de faire émerger des vérités métaphoriques par les procédés rhétoriques de l'hyperlien et l'animation textuelle. 
Le recours à certains termes issus de la rhétorique papier ne doit pas détourner l'attention de la spécificité de ces procédés : grâce à sa manipulabilité et à l'animation, le texte numérique implique le corps du lecteur, ses sensations et émotions de façon inédite. Nous ne sommes qu'au début de l'exploration de ce champ des possibles. Certains auteurs et éditeurs hésitent encore à le mettre en œuvre. Craignant que l'hyperlien perturbe la lecture, ils réduisent son utilisation au minimum. Craignant que l'animation incite le lecteur à seulement regarder le texte au lieu de le lire, les couplages entre texte et mouvement restent réservés aux champs restreints de la publicité numérique, du jeu vidéo et du livré enrichi pour enfants.

L'exploration restreinte de l'hyperlien et de l'animation s'explique sans doute, d'une part, par la méconnaissance des possibles ; d'autre part, elle s'enracine dans la vieille peur que le texte, mis en concurrence avec des formes faisant appel aux sens et sensations du lecteur, pourrait disparaître. Je n'ai, par exemple, repéré jusqu'alors aucun ciné-gramme ou kiné-gramme dans des revues scientifiques en ligne.

Il est certain que l'hyperlien comme l'animation peuvent encourager des pratiques extensives, immersives, voire compulsives. Au lieu de considérer ces pratiques comme une perte de raison, serait-il possible d'envisager que des vérités métaphoriques émergent dans l'expérience du vertige ? Pouvons-nous envisager l'écriture de textes journalistiques, voire de textes scientifiques qui mettent en œuvre l'expérience de la vitesse, de la superposition fragmentaire de traces mémorielles provoquée par le clic frénétique, et de la sensation de plongée jouissive engendrée par certaines animations?

Mieux encore peut-être, pouvons-nous commencer à concevoir des textes à entrées multiples anticipant à la fois sur des pratiques intensives, référentielles, extensives, depréhensives et non référentielles et sur leur hybridation, informant, faisant dialoguer, racontant, immergeant, affirmant ainsi par le sens et par le sensible?

Oui, décidément, nous ne sommes qu'au début de l'exploration de ce champ des possibles. Ce livre est une invitation à entreprendre cette exploration avec perspicacité et audace. 


\section{BIBLIOGRAPHIE}

Aarseth Espen, Cybertext. Perspectives on Ergodic Literature, Baltimore, The Johns Hopkins University Press, 1997.

Adorno Theodor W., « L'industrie culturelle », Conférence pour l'université radiophonique internationale, première diffusion les 21 et 28 septembre 1963. [En ligne] : < http://www.le-terrier.net/adorno/industrie. htm $>$.

Adorno Theodor W., Théorie esthétique, Paris, Klincksieck, 2004.

Amerika Mark, "Expanding the Concept of Writing. Notes on Net Art, Digital Narrative, and Viral Ethics”, Leonardo, 2004, vol. 37, no 1, pp. 9-13.

Anand Punam, Sternthal Brian, "Perceptual Fluency and Affect Without Recognition”, Memory \& Cognition, 1991, vol. 19, no 3, pp. 293-300.

Arcand Richard, Bourbeau Nicole, La communication efficace. De l'intention aux moyens d'expression, Bruxelles, DeBoeck, 1995.

Archibald Samuel, Le texte et la technique. La lecture à l'heure des médias numériques, Montréal, Le Quartanier, 2009.

Aristote, Poétique, Michel Magnien (trad.), Paris, Librairie générale française, 1990.

Baccino Thierry, « Lecture sur écran. Processus cognitifs. Point de vue de chercheurs en France », 2009. [En ligne] : < http://eduscol.education. fr/numerique/dossier/lectures/lecture-sur-ecran/processus-cognitifs/ points-de-vue-de-chercheurs-en-france/thierry-baccino >.

Baccino Thierry, "Lire sur Internet, est-ce toujours lire ? », Bulletin des bibliothèques de France, 2011, 5, pp. 63-66. [En ligne] : < http://bbf. enssib.fr/consulter/bbf-2011-05-0063-011 >.

Badir Sémir, « Valeur et variation, sémiologie et rhétorique », Semen, 2011, 32. [En ligne] : < http://semen.revues.org/9333 >. 
Bakhtine Mikhaill, La poétique de Dostoïevski, Paris, Seuil, 1970.

Baltz Claude, Documents de séminaire, Université Paris 8, 2009.

Baltz Claude, « La cyberculture : un driver obligé pour la société de l'information ? », Conférence au Congrès de la FADBEN, Nice, 8-10 avril 2005.

Baltz Claude, Information : Shannon en Questions. Retour Sur un Concept Majeur, Paris, Europia, 1999.

Barbier-Bouvet Jean-François, « La lecture de magazines par les jeunes adultes : un écran de papier ? », in Evans Christophe (dir.), Lectures et lecteurs à l'heure d'Internet. Livre, presse, bibliothèques, Paris, Éditions du Cercle de la Librairie, 2011, pp. 115-134.

Barthes Roland, «L'ancienne rhétorique », Communications, 1970, 16, pp. 172-229.

Barthes Roland, Sade, Fourier, Loyola, Paris, Seuil, 1971.

Barthes Roland, « Texte (théorie du) », Encyclopédie Universalis, 1973a, t. 15, p. 1015.

Barthes Roland, Le plaisir du texte, Paris, Seuil, 1973b.

Bautier Roger, De la rhétorique à la communication, Grenoble, Presses universitaires de Grenoble, 1994.

Béguin-Verbrugge Annette, " Information, communication et anthropologie des savoirs », RECIIS - Electronic Journal of Communication, Information and Innovation in Health, 2009, 3, 3. [En ligne] : < www.reciis. cict.fiocruz.br/ >.

Bélisle Claire (dir.), Lire dans un monde numérique, Villeurbanne, Presses de l'enssib, 2011 (coll. Papiers).

Bélisle Claire, "Conclusion », in Bélisle Claire (dir.), Lire dans un monde numérique, Villeurbanne, Presses de l'enssib, 2011 (coll. Papiers), pp. 277-289. 
Bélisle Claire, « Du papier à l'écran : lire se transforme », in Bélisle Claire (dir.), Lire dans un monde numérique, Villeurbanne, Presses de l'enssib, 2011 (coll. Papiers), pp. 111-162.

Bélisle Claire, « Introduction », in Bélisle Claire (dir.), Lire dans un monde numérique, Villeurbanne, Presses de l'enssib, 2011 (coll. Papiers), pp. 9-48.

Bélisle Claire, "Les nouveaux environnements de lecture ", in Bélisle Claire (dir.), Lire dans un monde numérique, Villeurbanne, Presses de l'enssib, 2011 (coll. Papiers), pp. 179-204.

Bélisle Claire (dir.), Alusse André, Belaïd Abdel, Chartron Ghislaine, Endrizzi Laure, Feyfant Annie, Limam Latifa, Lecointe Nicolas, Rangoni Yves, Rosado Eliana, Saemmer Alexandra, Scoccimarr Bruno, Rapport ELLEN : Encyclopédies en ligne et lectures numériques, Projet soutenu dans le cadre de l'appel à propositions « Usages de l'Internet » lancé conjointement par le ministère délégué à la Recherche et la DATAR, en liaison avec la Caisse des dépôts et consignations, 2006. [En ligne] : $<$ http://lire.ish-lyon.cnrs.fr/spip.php?rubrique88 >.

Bergk Johann Adam, Die Kunst, Bücher zu lesen. Nebst Bemerkungen über Schriften und Schriftsteller, Iéna, Hempel, 1799.

Bernstein Mark, "Patterns of hypertext”, 2003. [En ligne] : < http://www. eastgate.com/patterns/Print.html >.

Birkerts Sven, The Gutenberg Elegies. The Fate of Reading in an Electronic Culture, Winchester, Faber \& Faber, 1994.

Blanckeman Bruno, Les récits indécidables. Jean Echenoz, Hervé Guibert, Pascal Quignard, Villeneuve-d'Ascq, Presses universitaires du Septentrion, 2000 (coll. Perspectives. Lettres et arts).

Bollack Jean, Sens contre sens. Comment lit-on ? Entretien avec Patrick Llored, Lyon, La passe du vent, 2000.

Bolter Jay David, Writing Space. The Computer, Hypertext, and the History of Writing, Hillsdale, Lawrence Erlbaum, 1991.

Bolter Jay David, Grusin Richard, Remediation. Understanding New Media, Cambridge, The MIT Press, 2000. 
Bonhomme Marc, Pragmatique des figures de discours, Paris, Honoré Champion, 2005.

Bonhomme Marc, «Peut-on parler de métonymie iconique ? », in Badir Sémir, Klinkenberg Jean-Marie (dir.), Figures de la figure. Sémiotique et rhétorique générale, Limoges, Presses universitaires de Limoges, 2008, pp. 215-228.

Bootz Philippe, « Note », in Alire, 1990, 3.

Bootz Philippe (dir.), Alire, 12, Poésie et art programmés, avec : Jim Andrews, Wilton Azevedo, Jean-Pierre Balpe, Philippe Bootz, B-L-U-ES-C-R-E-E-N, Patrick Henry Burgaud, John Cayley, Philippe Castelin, Alexandre Gherban, Xavier Leton, Tibor Papp, Nazura Rahime, Antoine Schmitt, Eric Sérandour, Reiner Strasser, CD-ROM, Villeneuve-d'Ascq, Mots-Voir et les auteurs, 2004.

Bootz Philippe, Saemmer Alexandra, "Semiotic Cross Analyses of Digital Poetry", Intervention au Congrès international de la Electronic Literature Association, 20 juin 2012, West Virginia University, Morgantown.

Bordeleau Benoît, « Marcher la ville brisée », Intervention dans "Word and Image", $9^{e}$ Congrès international pour l'étude des rapports entre texte et image, 22 au 26 août 2011, UQAM, Montréal.

Borges Jorge Luis, Enquêtes, Paris, Gallimard, 1957 (coll. Du monde entier). Borges Jorge Luis, Fictions, Paris, Gallimard, 1965.

Bouchardon Serge, " Hypertexte et art de l'ellipse », in Ghitalla Franck (dir.), Les Cahiers du numérique, 3, La navigation, Paris, Hermès, 2002, pp. 65-86.

Bouchardon Serge, Littérature numérique. Le récit interactif, Paris, Hermès, 2009.

Bouchardon Serge, « Des figures de manipulation dans la création numérique », Protée, 2011, vol. 39, no 1, pp. 37-46.

Boucher Amélie, Ergonomie Web. Pour des sites Web efficaces, Paris, Eyrolles, 2011 (coll. Accès Libre). 
Boullier Dominique, " "Profils, alerte et vidéo" : de l'outre-lecture à la fin de la lecture ? ", in Evans Christophe (dir.), Lectures et lecteurs à l'heure d'Internet. Livre, presse, bibliothèques, Paris, Éditions du Cercle de la Librairie, 2011, pp. 41-58.

Bourassa Renée, Les fictions hypermédiatiques. Mondes fictionnels et espaces ludiques, Montréal, Le Quartanier, 2010.

Bourdieu Pierre, Chartier Roger, " La lecture : une pratique culturelle. Débat », in Chartier Roger (dir.), Pratiques de la lecture, Paris, Payot, 2003, pp. 277-306.

Boutaud Jean-Jacques, Veron Eliseo, Sémiotique ouverte. Itinéraires sémiotiques en communication, Paris, Hermès, 2007.

Boutaud Jean-Jacques, " Du sens, des sens. Sémiotique, marketing et communication en terrain sensible ", Semen, 2007, 23. [En ligne] : $<$ http://semen.revues.org/5011 >.

Bouveresse Jacques, Prodiges et vertiges de l'analogie, Paris, Raisons d'agir, 1999.

Bowker, "British University Students Still Crave Print", 2012. [En ligne] : < http://www.bowker.com/en-US/aboutus/press_room/2012/ pr_03152012.shtml >.

Breton Philippe, « La presse régionale entre le fait universel et le commentaire local », Études de communication, 1995, 17. [En ligne] : < http:// edc.revues.org/index2460.html >.

Breton Philippe, Proulx Serge, L'explosion de la communication, Paris, La Découverte, 1989.

Buci-Glucksmann Christine, Esthétique de l'éphémère, Paris, Galilée, 2003 (coll. Écritures/Figures).

Cardon Dominique, "Vertus démocratiques de l'Internet ", La vie des idées, 2009. [En ligne] : < http://www.laviedesidees.fr/spip. php?page $=$ auteur\&id_auteur $=0>$.

Cardon Dominique, La démocratie Internet. Promesses et limites, Paris, Seuil, 2010 (coll. La République des Idées). 
Carr Nicholas, "Is Google making us stupid?”, The Atlantic, 2008. [En ligne] : < http://www.theatlantic.com/magazine/archive/2008/07/isgoogle-making-us-stupid/6868/ >.

Cassin Barbara, Clayssen Virginie, Leclère Thierry, "Internet rend-il bête ? », Télérama, 23 juillet 2009, no 3106. [En ligne] : <http://www. telerama.fr/techno/internet-rend-il-bete,45486.php>.

Cavallo Guglielmo, Chartier Roger, « Introduction », in Cavallo Guglielmo, Chartier Roger (dir.), Histoire de la lecture dans le monde occidental, Paris, Seuil, 2001, pp. 7-51.

Certeau Michel de, L'invention du quotidien, t. 1, Arts de faire, Paris, Gallimard, 1990.

Charles Michel, Rhétorique de la lecture, Paris, Seuil, 1977.

Charney Davida, "The Effect of Hypertext on Processes of Reading and Writing", in Hilligoss Susan, Selfe Cynthia (dir.), Literacy and Computers. The Complications of Teaching and Learning with Technology, New York, Modern Language Association, 1994, pp. 238-263.

Chatonsky Gregory, "Contre l'immersion », 2008, < http://incident.net/ users/gregory/wordpress/26-contre-limmersion/comment-page-1 > (consulté le 12 janvier 2012).

Citton Yves, L'avenir des Humanités. Économie de la connaissance ou cultures de l'interprétation?, Paris, La Découverte, 2010.

Clément Jean, " Fiction interactive et modernité », 1994. [En ligne] : $<$ http://hypermedia.univ-paris8.fr/jean/articles/litterature.html >.

Clément Jean, « Hypertexte et complexité », Études françaises, 2000, vol. 36, no 2, pp. 39-57.

Clément Jean, « Hypertexte et fiction : une affaire de liens », 2004. [En ligne] : < hypermedia.univ-paris8.fr/jean/articles/Lien.pdf >.

Compagnon Antoine, La seconde main ou le travail de la citation, Paris, Seuil, 1979 (coll. Philosophie générale).

Courbet Didier, Communication médiatique : les apports de la psychologie sociale. Pour une pluralité épistémologique, théorique et méthodologique 
en SIC, Note de synthèse des travaux pour l'obtention de l'Habilitation à diriger des recherches en sciences de l'information et de la communication, Marseille, Université de Provence-Aix-Marseille 1, 2004.

Courbet Didier, Borde Amélie, Intartaglia Julien, Denis Sylvain, « L'influence non consciente des publicités vues furtivement et aussitôt oubliées : une méthode d'étude socio-cognitive appliquée à Internet », Questions de communication, 2004, 5, pp. 83-102.

Courbet Didier, Fouquet-Courbet Marie-Pierre, « Les processus psychologiques lors de la création publicitaire », Hermès, 2005, no 41, pp. 67-74.

CSA, "Les pratiques numériques des internautes », Enquête pour Observatoire Orange - Terrafemina, 2011. [En ligne] : < http://eduscol. education.fr/numerique/textes/rapports/societe-numerique/culturenumerique/2011/csa >.

Currie Gregory, The Nature of Fiction, Cambridge, Cambridge University Press, 1990.

Cusset François, French Theory. Foucault, Derrida, Deleuze et Cie et les mutations de la vie intellectuelle aux États-Unis, Paris, La Découverte, 2003.

Davallon Jean, Després-Lonnet Marie, Jeanneret Yves, Le Marec Joëlle, Souchier Emmanuël, "Introduction », in Souchier Emmanuël, Jeanneret Yves, Le Marec Joëlle (dir.), Lire, écrire, récrire. Objets, signes et pratiques des médias informatisés, Paris, Bibliothèque publique d'information, 2003 (coll. Études et recherche), pp. 17-40.

Davallon Jean, Noël-Cadet Nathalie, Brochu Danièle, "L'usage dans le texte : les "traces d'usage" du site Gallica ", in Souchier Emmanuël, Jeanneret Yves, Le Marec Joëlle (dir.), Lire, écrire, récrire. Objets, signes et pratiques des médias informatisés, Paris, Bibliothèque publique d'information, 2003, pp. 47-90.

De Montety Caroline, « Le magazine comme outil tactique de médiation pour les marques : une représentation sociale du lien marchand », in Actes du colloque international "L'organisation média », Lyon, Université Jean-Moulin Lyon 3, 2004, pp. 83-90.

Deleuze Gilles, Guattari Félix, Mille plateaux, Paris, Minuit, 1980. 
Deleuze Gilles, Cours du 5 janvier 1982. [En ligne] : < http://gallica.bnf.fr/ ark:/12148/bpt6k1282642 >.

Derrida Jacques, De la grammatologie, Paris, Minuit, 1967 (coll. Critique).

Desrichard Yves, "Accélération du livre », Bulletin des bibliothèques de France, 2011, 5, pp. 58-62. [En ligne] : < http://bbf.enssib.fr/consulter/ bbf-2011-05-0058-010 >.

DeWitt Scott Lloyd, "Defining Links", in DeWitt Scott Lloyd, Strasma Kip (dir.). Contexts, Intertexts and Hypertexts, Cresskill, Hampton Press, 1999, pp. 117-154.

Dolbeau-Bandin Cécile, « Le Monde et Le Monde.fr : de la nécessité d'un tiers », Les Cahiers du journalisme, 2008, no 18, pp. 184-207.

Donnat Olivier, « Lecture, livre et littérature. Évolution 1973-2008 », in Evans Christophe (dir.), Lectures et lecteurs à l'heure d'Internet. Livre, presse, bibliothèques, Paris, Éditions du Cercle de la Librairie, 2011, pp. 27-40.

Doueihi Milad, Pour un humanisme numérique, Paris, Seuil, 2011 (coll. La Librairie du xx $x^{e}$ siècle).

Drèze Xavier, Zufryden Fred, "Internet Advertising. The Medium is the Difference", Consumption, Markets and Culture, 2000, vol. 4, no 1, pp. 12-20.

Ducrot Oswald, Schaeffer Jean-Marie, Nouveau dictionnaire encyclopédique des sciences du langage, Paris, Seuil, 1995.

Dumais Fabien, L'appropriation d'un objet culturel. Une réactualisation des théories de C. S. Peirce à propos de l'interprétation, Montréal, Presses universitaires du Québec, 2010.

Eco Umberto, Lector in fabula, Paris, Le Livre de poche, 1985 (coll. Biblio Essais).

Eco Umberto, "Vegetal and Mineral Memory. The Future of Books", 2003. [En ligne] : < http://weekly.ahram.org.eg/2003/665/bo3.htm >.

eMarketer, "Is the Click Still King ?". [En ligne] : < http://www.emarketer. com/go/IAB_France.aspx >. 
Ensslin Astrid, Canonizing Hypertext. Explorations and Constructions, Londres, Bloomsbury Academic, 2007.

Enzensberger Hans Magnus, « Una modesta proposta per difendere la gioventù dalle opere di poesia », in Sulla piccola borghesia. Un " capriccio sociologico » seguito da altri saggi, Milan, Il Saggiatore, 1983.

Ertzscheid Olivier, Affordance (blog). [En ligne] : < http://affordance.typepad.com/ >.

Ertzscheid Olivier, Le lieu, le lien, le livre. Les enjeux cognitifs et stylistiques de l'organisation hypertextuelle, Thèse de doctorat, sous la direction de François-Charles Gaudard et Jo Link-Pezet, Toulouse, Université Toulouse 2, 2001.

Ertzscheid Olivier, Gallezot Gabriel, Boutin Éric, « PageRank : entre sérendipité et logiques marchandes », in Simonnot Brigitte, Gallezot Gabriel (dir.), L'entonnoir. Google sous la loupe des sciences de l'information et de la communication, Paris, C\&F éditions, 2009, pp. 113-138.

Estienne Yannick, Le journalisme après Internet, Paris, L'Harmattan, 2007 (coll. Communication et Civilisation).

Evans Christophe (dir.), Lectures et lecteurs à l'heure d'Internet. Livre, presse, bibliothèques, Paris, Éditions du Cercle de la Librairie, 2011.

Fabre Isabelle, Couzinet Viviane, «Désir, curiosité, culture informationnelle : l'organisation des savoirs au cœur de l'histoire des idées », The Canadian Journal of Information and Library Science / La Revue canadienne des sciences de l'information et de bibliothéconomie, 2008, 32, 3-4, < http://www.utpjournals.com/cjils/cjils.html > (consulté le 15 février 2011).

Faucilhon Joël, Rêveurs, marchands et pirates : que reste-t-il du rêve de l'Internet ?, Le Pré-Saint-Gervais, Le Passager clandestin, 2010.

Faure Nadine, Sassinot-Uny Laetitia, Étude qualitative Dialego pour les États généraux de la presse écrite, 2008. [En ligne] : < http://www. culture.gouv.fr/culture/actualites/conferen/albanel/egpequali.pdf >.

Festinger Leon, A Theory of Cognitive Dissonance, Stanford, Stanford University Press, 1957. 
Finkielkraut Alain, Soriano Paul, Internet, l'inquiétante extase, Paris, Mille et une nuits, 2001.

Fish Stanley, Is There a Text in this Class? The Authority of Interpretative Communities, Cambridge, Harvard University Press, 1980.

Flichy Patrice, L’imaginaire d'Internet, Paris, La Découverte, 2001.

Flichy Patrice, «La place de l'imaginaire dans l'activité technique : le cas d'Internet ", in Actes des Quatrièmes rencontres réseaux humains / Réseaux technologiques, 31 mai et $1^{\text {er }}$ juin 2002, Poitiers, CNDP, 2003, pp. 87-104. [En ligne] : < http://edel.univ-poitiers.fr/rhrt/document553. php >.

Fontanier Pierre, Les figures du discours, Paris, Flammarion, 1977.

Foucault Michel, Ceci n'est pas une pipe, Paris, Fata Morgana, 1973.

Fourmentraux Jean-Paul, Art et Internet. Les nouvelles figures de la création, Paris, CNRS éditions, 2005.

Franke Matthias, « Ästhetik und Technik. Literaturprojekte der Semiosphäre ». [En ligne] : < http://www.franke-matthias.de/wlbfctxt.htm >.

Frey Aline, Poitrenaud Sébastien, Tijus Charles, «Validation expérimentale de la pertinence cognitive des UST », Musimédiane, 2010, 5. [En ligne] : < http://www.musimediane.com/ >.

Frommer Franck, "Au doigt et à l'œil », Bulletin des bibliothèques de France, 2011, no 5, pp. 63-66. [En ligne] : < http://bbf.enssib.fr/consulter/bbf-2011-05-0074-014 >.

Gaschke Susanne, Klick. Strategien gegen die digitale Verdummung, Fribourg, Herder, 2009.

Gauthier Joëlle, « Zeit für die Bombe », Répertoire du nt2, 2009, < http:// nt2.uqam.ca/repertoire/zeit_f\%C3\%BCr_die_bombe > (consulté le 13 juillet 2012).

Genette Gérard, "La rhétorique restreinte ", Communications, 1970, 16, pp. 158-171. 
Genette Gérard, Palimpsestes. La littérature au second degré, Paris, Seuil, 1982.

Genette Gérard, Nouveau discours du récit, Paris, Seuil, 1983.

Gervais Bertrand, Lecture littéraire et explorations en littérature américaine, Montréal, XYZ, 1998 (coll. Théorie et Littérature).

Gervais Bertrand, « Naviguer entre le texte et l'écran : penser la lecture à l'ère de l'hypertextualité », in Salaün Jean-Michel, Vandendorpe Christian (dir.), Les défis de la publication sur le Web : hyperlectures, cybertextes et méta-éditions, Villeurbanne, Presses de l'enssib, 2004.

Gervais Bertrand, " Richard Powers et les technologies de la représentation. Des vices littéraires et de quelques frontières », Alliage. Culture, science, technique, 2006, vol. 57-58, pp. 226-237.

Gervais Bertrand, Figures, lectures : logiques de l'imaginaire, t. 1, Montréal, Le Quartanier, 2007 (coll. Erres essais).

Gervais Bertrand, Guilet Anaïs, « Esthétique et fiction du flux. Éléments de description », Protée, 2011, 39, 1, pp. 89-100.

Gervais Bertrand, Xanthos Nicolas, «L'hypertexte : une lecture sans fin », 2003, < http://www.arts.uottawa.ca/astrolabe/articles/art0036.htm > (consulté le 4 mars 2010).

Giffard Alain, «Critique de la lecture numérique : The Shallows de Nicholas Carr », Bulletin des bibliothèques de France, 2011, no 5, pp. 63-66. [En ligne] : < http://bbf.enssib.fr/consulter/bbf-2011-05-0071-013 >.

Golson Emily, "Cognition, Meaning, and Creativity: On Reading Student Hypertexts”, in DeWitt Scott Lloyd, Strasma Kip (dir.), Contexts, Intertexts and Hypertexts, Cresskill, Hampton Press, 1999, pp. 155-176.

Grafton Anthony, « Le lecteur humaniste », in Cavallo Guglielmo, Chartier Roger (dir.), Histoire de la lecture dans le monde occidental, Paris, Seuil, 2001, pp. 221-264.

Greimas Julien Algirdas, Courtés Joseph, Dictionnaire raisonné de la théorie du langage, Paris, Hachette, 1986, t. 2. 
Gumbrecht Hans Ulrich, Éloge de la présence. Ce qui échappe à la signification, Stanford, Stanford University Press, 2004 [pour le titre original] ; Paris, Libella Maren Sell, 2010 [pour la traduction française].

Hamesse Jacqueline, « Le modèle scholastique de la lecture », in Cavallo Guglielmo, Chartier Roger (dir.), Histoire de la lecture dans le monde occidental, Paris, Seuil, 2001, pp. 131-152.

Hautzinger Nina, Vom Buch zum Internet? Eine Analyse der Auswirkungen hypertextueller Strukturen auf Text und Literatur, Mannheimer Studien zur Literatur- und Kulturwissenschaft, 1999. Extraits : [en ligne] : < http://www.berlinerzimmer.de/eliteratur/hautzinger/hautz3. htm\#top $>$.

Hayles N. Katherine, "Hyper and Deep Attention: the Generational Divide in Cognitive Modes”, Profession, 2007, pp. 187-199.

Heller Eva, Psychologie de la couleur. Effets et symboliques, Paris, Pyramyd, 2009.

Hersent Jean-François, "Sociologie de la lecture en France : état des lieux », 2000. [En ligne] : < http://www.culture.gouv.fr/culture/dll/ sociolog.rtf $>$.

Huesca Robert, Dervin Brenda, "Hypertext and Journalism”, in Jenkins Henry, Thorburn David (dir.), Democracy and New Media, Cambridge, MIT Press, 2003, pp. 281-307.

Hugo Victor, Voyages, Paris, Laffont, 1987.

IAB Europe, " 100 milliards d'euros de services Internet financés par la pub en ligne ». [En ligne] : < http://www.iabfrance.com/getfile. php?id=2833 >.

Ihadjanene Madjid, La recherche et la navigation dans un système de recherche d'information grand public, Thèse de doctorat, sous la direction de Richard Bouché, Lyon, Université Lyon 1, 1999.

Iser Wolfgang, L'acte de lecture. Théorie de l'effet esthétique, Munich, Fink, 1976 [pour le titre original] ; Bruxelles, Mardaga, 1995 [pour la traduction française citée]. 
Jahjah Marc, «À la recherche désespérée de la lecture asociale (I) : discours et représentations », 2011, < http://www.sobookonline.fr/livrenumerique/livre-social/a-la-recherche-desesperee-de-la-lecture-asociale/ > (consulté le 15 mai 2012).

Jakobiak François, L’information scientifique et technique, Paris, PUF, 1995.

Jauss Hans Robert, Pour une esthétique de la réception, Munich - Francfort-sur-le-Main, Fink - Suhrkamp et al., 1972-1978 [pour le titre original] ; Paris, Gallimard, 2010, préface Jean Starobinski [pour la traduction française citée].

Jeanneret Yves, Y a-t-il (vraiment) des technologies de l'information?, Paris, Presses universitaires du Septentrion, 2007a.

Jeanneret Yves, " La prétention sémiotique dans la communication. Du stigmate au paradoxe », Semen, 2007b, 23. [En ligne] : < http://semen. revues.org/8496 >.

Jeanneret Yves, Penser la trivialité, vol. 1, La vie triviale des êtres culturels, Paris, Hermès-Lavoisier, 2008.

Jeanneret Yves, Davallon Jean, « La fausse évidence du lien hypertexte », Communication et langages, 2004, no 140, pp. 43-54.

Jeanneret Yves, Béguin Annette, Cotte Dominique, Labelle Sarah, Perrier Valérie, Quinton Philippe, Souchier Emmanuël, « Formes observables, représentations et appropriation du texte de réseau ", in Souchier Emmanuël, Jeanneret Yves, Le Marec Joëlle (dir.), Lire, écrire, récrire. Objets, signes et pratiques des médias informatisés, Paris, Bibliothèque publique d'information, 2003, pp. 91-158.

Jeanneret Yves, Souchier Emannuël, " Pour une poétique de l'écrit d'écran », Xoana, 1998, 6, pp. 97-107.

Jeanneret Yves, Souchier Emannuël, « L'énonciation éditoriale dans les écrits d'écran », Communication et langages, 2005, no 145, pp. 3-15.

Jennings Pamela, "Narrative Structures for New Media. Towards a New Definition”, Leonardo, 1996, vol. 29, no 5, pp. 345-350.

Kahane Howard, Logic and Contemporary Rhetoric. The Use of Reason in Everyday Life, Belmont, Wadsworth, 1976. 
Kapferer Jean-Noël, Laurent Gilles, « Les profils d'implication », Recherche et applications en marketing, 1986, no 1, pp. 3-20.

Kelly Kevin, “An Intermedia with 2 Billion Screens Peering into it”, 2010, < http://www.edge.org/q2010/q10_1.html\#kelly > (consulté le 15 mai 2012).

Kleltz Françoise, "La lecture des étudiants en sciences humaines et sociales », Cahiers de l'économie du livre, 1992, 7, pp. 5-57.

Klinkenberg Jean-Marie, Précis de sémiotique générale, Bruxelles, De Boeck, 1996.

Kristeva Julia, Sèméiôtikè. Recherches pour une sémanalyse, Paris, Seuil, 1969 (coll. Essais).

Labelle Sarah, " "La société de l'information”, à décrypter ! ", Communication et langages, 2001, no 128, pp. 65-79.

Laboratoire Musique et informatique de Marseille, "Les unités sémiotiques temporelles ». [En ligne] : < http://www.labo-mim.org/site/ index.php?2008/08/22/44-liste-des-19-ust >.

Laborde Aurélie (dir.), « Quel imaginaire d'Internet dans la société française ? Représentations d'Internet dans la presse généraliste depuis 2000 », Rapport de recherche, 2010. [En ligne] < http://raudin.u-bordeaux3.fr/oat/wp-content/uploads/SYNTHESE\%20IMAGINAIRE\%20D। x27INTERNET.pdf >.

Landow George P., Hypertext 2.0. The Convergence of Contemporary Critical Theory and Technology, Baltimore, The Johns Hopkins University Press, 1997.

Landow George P., Hypertext 3.0. Critical Theory and New Media in an Era of Globalization, Baltimore, The Johns Hopkins University Press, 2006.

Landowski Éric, Passions sans nom, Paris, PUF, 2004 (coll. Formes sémiotiques).

Laroche Hervé, "Information, technologie et domination », Les Échos, 1999, < http://www.lesechos.fr/formations/manag_info/articles/article_11_3.htm > (consulté le 13 mai 2011). 
Latour Bruno, "La rhétorique scientifique : qu'est-ce que la force d'un argument ? », in Sens et place des connaissances dans la société, Paris, CNRS éditions, 1986.

Latour Bruno, Matériaux pédagogique pour la controverse structurée, $<$ http://www.bruno-latour.fr/cours/index.html > (consulté le 20 décembre 2010).

Le Deuff Olivier, Le guide des égarés (blog). [En ligne] : < http://www.guidedesegares.info/author/admin/ >.

Le Grignou Brigitte, Neveu Erik, « Émettre la réception. Préméditation et réception de la politique télévisée », Réseaux, 1988, 32, pp. 67-107.

Le Groupe $\mu$, Traité du signe visuel, Paris, Seuil, 1992.

Le Marec Joëlle, Babou Igor, "De l'étude des usages à une théorie des "composites" : objets, relations et normes en bibliothèque ", in Souchier Emmanuël, Jeanneret Yves, Le Marec Joëlle (dir.), Lire, écrire, récrire. Objets, signes et pratiques des médias informatisés, Paris, Bibliothèque publique d'information, 2003 (coll. Étude et recherche), pp. 233-296.

Léger Fernand, Fonctions de la peinture, Paris, Gonthier, 1965.

Lendrevie Jacques, « Internet est-il doué pour la publicité ? », Revue française de marketing, 2000, no 177-178, pp. 102-118.

Lévy Pierre, Qu'est-ce que le virtuel ?, Paris, La Découverte, 1998.

Lévy Pierre, Cyberculture, Paris, Odile Jacob, 1997.

Li Chan, Poe Felicia, Potter Michele, Quigley Brian, Wilson Jacqueline, UC Libraries Academic e-Book. Usage Survey, Springer e-Book Pilot Project, University of California Projects, 2011. [En ligne] : < http://www. cdlib.org/services/uxdesign/docs/2011/academic_ebook_usage_survey.pdf $>$.

Magné Bernard, « Machines à écrire, machine à lire », Études françaises, 2000, vol. 36, no 2, pp. 119-128.

Manovich Lev, The Language of New Media, Cambridge, MIT Press, 2001. 
Marino Mark, "Critical code studies », The Electronic Book Review, 2006. [En ligne] : < http://www.electronicbookreview.com/thread/electropoetics/codology/ >.

Mattelart Armand et Michèle, Histoire des théories de la communication, Paris, La Découverte, 2004.

McLuhan Marshall, Understanding Media. The Extensions of Man, New York, McGraw-Hill Book Company, 1964.

Mettout Éric, « Le petit manuel du parfait stagiaire (et réciproquement) », 2010. [En ligne] : < http://blogs.lexpress.fr/nouvelleformule/2010/07/27/ le_petit_manuel_du_parfait_sta/ >.

Meunier Jean-Pierre, "Pour une approche cognitive de la signification iconique », in Darras Bernard (dir.), Images et sémiotique. Sémiotique pragmatique et cognitive, Paris, Publications de la Sorbonne, 2006 (coll. Esthétique), pp. 133-145.

Miège Bernard, L'espace public contemporain, Grenoble, Presses universitaires de Grenoble, 2010.

Ministère de la Culture et de la Communication, «Enquêtes sur les "Pratiques culturelles des Français”", depuis 1973. [En ligne] $:<$ http:// www.pratiquesculturelles.culture.gouv.fr/ >.

Moles Abraham, Informationstheorie und ästhetische Wahrnehmung, Cologne, Du Mont [pour la traduction allemande citée], 1971.

Monnoyer-Smith Laurence, « La participation en ligne, révélateur d'une évolution des pratiques politiques ? », Participations, 2011, no 1, Démocratie et participation : un état des savoirs, pp. 156-185.

Montfort Nick, Strickland Stephanie, "Commenting Creative Code”, Intervention au Congrès international de la Electronic Literature Association, 20 juin 2012, West Virginia University, Morgantown, 2012.

Musil Robert, Gesammelte Werke, Reinbek bei Hamburg, Rowohlt, 1978.

Musil Robert, L'homme sans qualités, Philippe Jaccottet (trad.), Paris, Seuil, 1956.

Nelson Theodore Holm, Literary Machines, Sausalito, Mindful Press, 1982. 
Nibley Andrew, "The Internet and the New Generation of Newsreaders", Historical Journal of Film, Radio and Television, 2000, vol. 20, no 1, pp. 37-42.

Nielsen Jakob, Designing Web Usability, Berkeley, Peachpit Press, 1999.

Nielsen Jakob, Loranger Hoa, Site Web. Priorité à la simplicité, Paris, Campus Press, 2007.

Nietzsche Friedrich, Le livre du philosophe, Angèle K. Marietti (trad.), Paris, Flammarion, 1993 (coll. GF Philosophie).

Nolan Sybil, "Journalism Online. Search for Narrative Form in a Multilinear Word", in Melbourne DAC, the 5th International Digital Arts and Culture Conference, 19-23 mai 2003. [En ligne] : < hypertext.rmit.edu. $\mathrm{au} /$ dac/papers/Nolan.pdf >.

Norton David, Zimmerman Beverly, Lindeman Neil, "Developing Hyperphoric Grammar to Teach Collaborative Hypertexts", in DeWitt Scott Lloyd, Strasma Kip (dir.), Contexts, Intertexts and Hypertexts, Cresskill, Hampton Press, 1999, pp. 177-202.

Observatoire de la vie étudiante (OVE), Enquête « Conditions de vie », 2006. [En ligne] : < http://www.ove-national.education.fr/enquete/2006 >.

Oléron Pierre, L'argumentation, Paris, PUF, 1983.

Pajares Tosca Susana, “A Pragmatic of Links”, JoDI, 27 juin 2000, 1, 6.

Pélissier Nicolas et al., « Tous journalistes ? Les établissements de formation au défi du Web participatif », Les cahiers du journalisme, 2010, 21, pp. 176-190.

Pearson, "New Survey Finds Dramatic Increase in Tablet Ownership Among College Students and High School Seniors", 2012. [En ligne] : < www.pearsonfoundation.org/pr/20120314-new-survey-finds-dramatic-increase-in-tablet-ownership-among-college-students-and-highschool-seniors.html >.

Perelman Chaïm, L'empire rhétorique. Rhétorique et argumentation, $2^{\mathrm{e}}$ éd., Paris, Vrin, 2009. 
Perelman Chaïm, Olbrechts-Tyteca Lucie, Traité de l'argumentation. La nouvelle rhétorique, Bruxelles, Éditions de l'université de Bruxelles, 1970.

Perriault Jacques, « Effet diligence, effet serendip et autres défis pour les sciences de l'information », 2000. [En ligne] : < http://archives.limsi.fr/ WkG/PCD2000/textes/perriault.html >.

Petrucci Armando, « Lire pour lire : un avenir pour la lecture », in Cavallo Guglielmo, Chartier Roger (dir.), Histoire de la lecture dans le monde occidental, Paris, Seuil, 2001, pp. 341-358.

Pignier Nicole, « Analyse sémiotique de la webpublicité », Semiotica, 2005, vol. 157, no 1-4, pp. 521-538.

Pignier Nicole, Drouillat Benoît, Le webdesign. Sociale expérience des interfaces Web, Paris, Hermès, 2008 (coll. Forme et sens).

Pimentel Ken, Teixeira Kevin, Virtual Reality. Through the New Looking Glass, New York, Intel/Windcrest - McGraw Hill, 1993.

Pinte Jean-Paul, La veille informationnelle en éducation pour répondre au défi de la société de la connaissance au XXI siècle. Application à la conception d'une plateforme de veille et de partage de connaissances en éducation : Commun@utice, sous la direction de Clément Paoli et Henri Dou, Marne-la-Vallée, Université de Marne-la-Vallée, 2006. [En ligne] : < http://lewebpedagogique.com/cultureinformationnelle/ page/3/ >.

Proust Marcel, Sur la lecture, Arles, Actes Sud, 1993.

Quintilien, Institution oratoire, livres VIII et IX, Paris, Les Belles Lettres, 1978.

Rastier François, Arts et sciences du texte, Paris, PUF, 2001.

Rebillard Franck, Touboul Annelise, "Promises Unfulfilled ? Journalism 2.0, User Participation and Editorial Policy on Newspaper Websites”, Media, Culture and Society, 2010, vol. 32, no 2, pp. 323-334.

Rheingold Howard, The Virtual Community. Homesteading on the Electronic Frontier, Cambridge, MIT Press, 2000. 
Ricœur Paul, Temps et récit, t. 1, L’intrigue et le récit historique, Paris, Seuil, 1983.

Riffaterre Michael, « La trace de l'intertexte », La pensée, no 215, 1980, pp. 4-19.

Robbe-Grillet Alain, Temps et description dans le récit d'aujourd'hui. Pour un nouveau roman, Paris, Gallimard, 1963 (coll. Idées).

Robbe-Grillet Alain, Projet pour une révolution à New York, Paris, Minuit, 1970.

Roque Georges, "À propos du Traité du signe visuel : une remarque et deux questions ", in Nouveaux actes sémiotiques, Actes du colloque de 2008, Le Groupe $\mu$. Quarante ans de rhétorique - trente-trois ans de sémiotique visuelle, 2010, < http://rfevues.unilim.fr/nas/document. php?id=3290 > (consulté le 2 février 2012).

Roque Georges, « Rhétorique visuelle et argumentation visuelle », Semen, 2011, 32. [En ligne] : < http://semen.revues.org/9370 >.

Rosario Giovanna di, Electronic Poetry. Understanding Poetry in the Digital Environment, Thèse de doctorat, Finlande, Université de Jyväskylä.

Rosnay Joël de, L'homme symbiotique. Regards sur le troisième millénaire, Paris, Seuil, 1995.

Runkehl Jens, Schlobinski Peter, Siever Torsten, Sprache und Kommunikation im Internet, Wiesbaden, Westdeutscher Verlag, 1998.

Ryan Marie-Laure, Avatars of Store, Minneapolis, University of Minnesota Press, 2006.

Ryan Marie-Laure, Narrative as Virtual Reality, Baltimore - Londres, The Johns Hopkins University Press, 2001.

Saemmer Alexandra, Duras et Musil - drôle de couple ? Drôle d'inceste ?, Amsterdam - New York, Rodopi, 2002.

Saemmer Alexandra, "La Révolution hypertextuelle », in Colin RenéPierre (dir.), Écrire la rupture, Tusson, Du Lerot, 2003, pp. 191-203. 
Saemmer Alexandra, "Courriers de lecteurs à propos de "Sublime, forcément sublime Christine V." de Marguerite Duras », in Cousseau Anne, Denès Dominique (dir.), Marguerite Duras. Marges et transgressions, Nancy, Presses universitaires de Nancy, 2006, pp. 149-157.

Saemmer Alexandra, Matières textuelles sur support numérique, SaintÉtienne, Publications de l'université de Saint-Étienne, 2007.

Saemmer Alexandra, "Figures de surface média », Protée, 2008, 36, 1, pp. 79-90.

Saemmer Alexandra, "Digital Literature. A Question of Style", in Simanowski Roberto, Schäfer Jörgen, Gendolla Peter, Reading Moving Letters. Digital Literature in Research and Teaching. A Handbook, Bielefeld, Transcript Verlag, 2009, pp. 163-182.

Saemmer Alexandra, "Les "effets" préfigurés par l'outil de conception numérique - stéréotypes, clichés ou contraintes libératrices ? », in Yacoub Claude, Zreik Khaldoun (dir.), Conception assistée par concepteur, Paris, Europia, 2010a, pp. 157-162.

Saemmer Alexandra, "Approche semio-rhétorique des couplages textemouvement dans le discours numérique ", in Ihadjanene Madjid, Zackland Manuel, Zreik Khaldoun (dir.), Cide 13, Paris, Europia, 2010b, pp. 25-38.

Saemmer Alexandra, " De la confirmation à la subversion : les figures d'animation face aux conventions du discours numérique ", Protée, 2011a, vol. 39, no 1, pp. 23-36.

Saemmer Alexandra, "Writing the Ephemeral and Re-Enchanting the Remnants. The Lability of the Digital Device in Literary Practice", Journal of Writing in Creative Practice, 2011b, 4, 1, Intellect Journals, pp. 79- 92.

Saemmer Alexandra, " La littérature numérique entre légitimation et canonisation », Culture et musées, 2011c, 18, pp. 201-223.

Saemmer Alexandra, "Irradiations iconiques : réflexions sur le texte animé, à partir de quatre exemples dans la littérature numérique », Nouveaux actes sémiotiques, 2012, 115, Analyses sémiotiques, < http:// revues.unilim.fr/nas/document.php?id=4970 > (consulté le 4 août 2012). 
Saemmer Alexandra, Maza Monique, E-Formes 2. Les littératures et arts numériques au risque du jeu, Saint-Étienne, Publications de l'université de Saint-Étienne, 2011.

Saussure Ferdinand de, Écrits de linguistique générale, Paris, Gallimard, 2002 (coll. Bibliothèque de philosophie).

Shannon Claude E., "A Mathematical Theory of Communication”, Bell System Technical Journal, 1948, 27, pp. 379-423, pp. 623-656.

Shapiro Stewart, Krishnan H. Shanker, "Memory-based Measures for Assessing Advertising Effects. A Comparison of Explicit and Implicit Memory Effects”, The Journal of Advertising, 2001, 30, pp. 1-14.

Simanowski Roberto, "Susanne Berkenhegers “Zeit für die Bombe”. Die Tat des Lesers, die simulierte Zerstörung und die Redundanz der Animation », Dichtung digital, 1999. [En ligne] < http://dichtung-digital. mewi.unibas.ch/Simanowski/2-Juli-99/brief1_0_x.htm >.

Simanowski Roberto, « Hyperfiction pur, Interview mit Susanne Berkenheger », 2000. [En ligne] : < http://berkenheger.netzliteratur.net/ouargla/home/interview1.htm $>$.

Simanowski Roberto, "What is and to What End Do We Read Digital Literature?”, Dichtung digital, 2007, < http://dichtung-digital.mewi.unibas. ch/readingdigitalliterature/proceedings-Simanowski.htm > (consulté le 12 décembre 2011).

Simanowski Roberto, Digitale Medien in der Erlebnisgesellschaft, Reinbek bei Hamburg, Rowohlt, 2008.

Simon Claude, « Un homme traversé par le travail. Entretien », La nouvelle critique, 1977, 105, pp. 32-43.

Souchier Emmanuël, « L'image du texte. Pour une théorie de l'énonciation éditoriale », Les cahiers de médiologie, 1998, no 6, pp. 137-145.

Stenger Thomas, Coutant Alexandre (dir.), « Ces réseaux numériques dits sociaux », Hermès, 2011, 59.

Sterne Laurence, The Life and Opinions of Tristram Shandy, Gentleman, Londres, Penguin Classics, 2003. 
Stein Bob, « A Taxonomy of Social Reading. A Proposal », 2010. [En ligne] < http://futureofthebook.org/social-reading/introduction/ >.

Stiegler Bernard, La technique et le temps, Paris, Galilée, 2001, t. 3.

Stiegler Bernard, « La formulation de la nouvelle raison. Sept propositions pour l'école », in Kambouchner Denis, Meirieu Philippe, Stiegler Bernard, Gautier Julien, Vergne Guillaume, L'école, le numérique et la société qui vient, Paris, Mille et une nuits, 2012, pp. 197-201.

Stiegler Bernard, Kambouchner Denis, Meirieu Philippe, «Écriture et numérique. Entretien I », in Kambouchner Denis, Meirieu Philippe, Stiegler Bernard, Gautier Julien, Vergne Guillaume, L'école, le numérique et la société qui vient, Paris, Mille et une nuits, 2012, pp. 19-48.

Stiegler Bernard, Kambouchner Denis, Meirieu Philippe, «L'école dans la "société de la connaissance". Entretien II », in Kambouchner Denis, Meirieu Philippe, Stiegler Bernard, Gautier Julien, Vergne Guillaume, L'école, le numérique et la société qui vient, Paris, Mille et une nuits, 2012, p. 49-74.

Suter Beat (2005), « Narrationspfade in Hyperfictions, Erzählung als Weg durch den fiktiven Raum ». [En ligne] : < http://www.netzliteratur.net/ suter/narrationspfade.htm $>$.

Svenbro Jesper, « La Grèce archaïque et classique. L'invention de la lecture silencieuse », in Cavallo Guglielmo, Chartier Roger (dir.), Histoire de la lecture dans le monde occidental, Paris, Seuil, 2001, pp. 51-84.

The Economist, "All too much", 27 février 2010, < http://www.economist. com/node/1555742 > (consulté en 2011).

Tétu Jean-François, « Sur les origines littéraires des sciences de l'information et de la communication », in Boure Robert (dir.), Les origines des sciences de l'information et de la communication. Regards croisés, Villeneuve-d'Ascq, Presses universitaires du Septentrion, 2002, pp. 71-93.

Todorov Tzvetan, « Les poètes devant le bon usage », Revue d'esthétique, 1965, vol. 18, no 3-5, pp. 300-305. 
Van der Klei Alice, « De l'utilité du rhizome pour penser l’hypermédia », Intervention dans "Histoire et archives », colloque international, 30 avril-2 mai 2009, Montréal, UQAM, 2009.

Vandendorpe Christian, Du papyrus à l'hypertexte. Essai sur les mutations du texte et de la lecture, Paris, La Découverte, 1999 (coll. Cahiers libres).

Vandendorpe Christian, «Quelques questions clés que pose la lecture sur écran », in Bélisle Claire (dir.), Lire dans un monde numérique, Villeurbanne, Presses de l'enssib, 2011 (coll. Papiers), pp. 49-66.

Varela Francisco J., Connaître les sciences cognitives. Tendances et perspectives, Pierre Lavoie (trad.), Paris, Seuil, 1989.

Vovou Ionna, "L'espace public médiatique vu comme un jeu à la télévision », in 18 Congrés de l'AISLF, 7-11 juillet 2008, Istanbul, Actes des travaux du groupe de travail Sociologie de la communication, 2008, pp. 226-233. [En ligne] : < http://w3.aislf.univtlse2.fr/gtsc/DOCS_ SOCIO/istambul/Actes_AISLF_GT13_ISTAMBUL_2008.pdf >.

Weissberg Jean-Louis, Présences à distance, déplacement virtuel et réseaux numériques : pourquoi nous ne croyons plus la télévision, Paris, L'Harmattan, 1999 (coll. Communication et civilisation).

Wittmann Reinhard, « Une révolution de la lecture à la fin du XVIII ${ }^{\mathrm{e}}$ siècle ? ”, in Cavallo Guglielmo, Chartier Roger (dir.), Histoire de la lecture dans le monde occidental, Paris, Seuil, 2001, pp. 355-392.

Wolfe Tom, “The New Journalism”, in Wolfe Tom, Johnson E. W. (dir.), The New Journalism, New York, Harper and Row, 1973, pp. 1-52.

Wolton Dominique, Informer n'est pas communiquer, Paris, CNRS éditions, 2009 (coll. Débats).

Zinna Alessandro, L'invention de l'hypertexte, Documents de travail, Urbino, Università di Urbino, 2002 (coll. Documents de travail ; no 318), p. 3. 


\section{EUVRES DE LITTÉRATURE ET D'ART NUMÉRIQUES CITÉES}

Berkenheger Susanne, Zeit für die Bombe, 1997. [En ligne] : < http://www. wargla.de/zeit.htm >.

Bon François, Le tiers livre. [En ligne] : < http://www.tierslivre.net/ >.

Bootz Philippe, Petits poèmes à lecture inconfortable. [En ligne] : < http:// epoetry.paragraphe.info/artists/oeuvres/bootz/bootz_index.htm >.

Bouchardon Serge, Déprise. [En ligne] : < http://lossofgrasp.com/ >.

Campbell Andy, Inside - a Journal of Dreams. [En ligne] $:<$ http://www. dreamingmethods.com/inside/ >.

Chatonsky Gregory, La Révolution à New York a eu lieu. [En ligne] : < http:// www.incident.net $>$.

Joyce Michael, Afternoon, a Story, Watertown, Eastgate Systems, 1993.

Saemmer Alexandra, "Tramway », bleuOrange, 2009, 2. [En ligne]: < http://revuebleuorange.org/bleuorange/02/saemmer/ >.

Saemmer Alexandra, Flux. [En ligne] : < http://www.mandelbrot.fr/flux. html $>$.

Stefans Brian Kimb, The Dreamlife of Letters. [En ligne] : < http://collection.eliterature.org/1/works/stefans_the_dreamlife_of_letters/ dreamlife_index.html >. 


\section{LISTE DES ILLUSTRATIONS}

Figure 1. Extrait de l'hyperfiction Zeit für die Bombe de Susanne Berkenheger

Figure 2. Capture d'écran d'un extrait de l'article de Wikipédia consacré aux disjoncteurs

Figure 3. Le mot « soldes » change de couleur ou de taille, à un rythme rapide et de façon réitérée

Figure 4. Capture d'écran d'un extrait d'Inside - a journal of dreams par Andy Campbell

Encadré 1. Affaire Galliano : Dior peut-il licencier Galliano pour des propos racistes?

Figure 5. Le haut et le milieu d'une page-écran de marianne2.fr

Figure 6. Le haut (à gauche) et le milieu (à droite) d'une page-écran de lexpress.fr

Figure 7. Le haut (à gauche) et le milieu (à droite) d'une page-écran du post.fr

Tableau 1. Formes-modèles de la page-écran et du texte

Figure 8. Exemple d'un hyperlien potentiellement « informationnel » dans l'article (13) du corpus, qui rentre dans la catégorie de l'hypertexte « définissant»

Figure 9. Exemple d'un hyperlien « dialogique » dans l'article (1) du corpus, qui rentre dans la catégorie de l'hypertexte " croisant les points de vue »

Figure 10. Exemple dans l'article (13) d'un hypertexte " croisant les points de vue»

Figure 11. Exemple d'un hyperlien qui entre dans la catégorie du « kiné-gramme »

Figure 12. Exemple d'un hyperlien " déformatoire » dans l'article (12) du corpus

Tableau 2. Figures de la lecture informationnelle

Tableau 3. Figures de la lecture dialogique

Tableau 4. Figures de la lecture immersive

Tableau 5. Figures de la lecture déviative

Tableau 6. Figures de la lecture informationnelle $67 \%$ 
Tableau 7. Figures de la lecture dialogique $29 \%$

Tableau 8. Figures de la lecture déviative $5 \%$

Tableau 9. Figures de la lecture immersive 2 (1\%)

Encadré 2. Face aux crises migratoires, Bruxelles est dans les choux

Figure 13. Captures d'écran d'extraits de l'article (22) paru sur marianne2.fr

Tableau 10. Article $22<$ http://www. marianne2.fr/Face-aux-crises-migratoires-Bruxelles-est-dans-leschoux_a203368.html >

Tableau 11. Identification des figures de la lecture

Encadré 3. Méthodologie d'analyse d'un texte numérique avec hyperliens

Figure 14. Capture d'une page-écran de Zeit für die Bombe de Susanne Berkenheger

Tableau 12. Formes-modèles de la page-écran et du texte

Figure 15. Extrait de Zeit für die Bombe
Tableau 13. Figures de la lecture de l'hyperlien et de l'animation textuelle et leurs procédés

Figure 16. Capture d'écran d'un extrait du site Agentdunet

Figure 17. Bannière publicitaire publiée dans une collection de journaldunet

Figure 18. Bannière publicitaire publiée dans une collection de journaldunet

Figure 19. Capture d'écran d'un extrait du site Kenzo parfums qui n'est plus visible à l'heure actuelle (2013)

Figure 20. Bannière publicitaire publiée dans une collection de journaldunet

Tableau 14. Formes-modèles pro-référentielles du texte animé

Tableau 15. Figures de la lecture du texte animé et leurs procédés

Tableau 16. Formes-modèles du texte et de la page-écran

Tableau 17. Figures de la lecture informationnelle 
Secrétariat d'édition:

Silvia Ceccani

Mise en page:

Cédric Vigneault

Conception graphique:

atelier Perluette, 69001 Lyon.

< http://www.perluette-atelier.com >

Achevé d'imprimer en janvier 2015

imprimerie Bialec (Nancy) - nº 84939

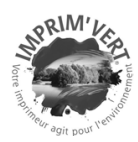

dépôt légal: $1^{\mathrm{er}}$ semestre 2015 


\section{PAPIERS SOUS LA DIRECTION DE

La collection Papiers a pour ambition d'explorer de nouveaux champs de recherche autour des sciences de l'information et des bibliothèques. Elle donne aux auteurs l'occasion de produire une réflexion nouvelle, originale, et propose de nouvelles lectures des domaines d'expertise de l'enssib.

\section{PRESSES DE L'ENSSIB}

École nationale supérieure des sciences de l'information et des bibliothèques Université de Lyon

17-21 boulevard du 11 novembre 1918

69623 Villeurbanne Cedex

Tél. 0472444343 - Fax 0472444344

Pour suivre l'actualité de nos

publications :

$<$ http://www.enssib.fr/presses >.

Pour obtenir la version imprimée (38 €) : commande auprès des libraires ou sur la librairie en ligne Le comptoir des presses d'universités (LCDPU)

< http://www.lcdpu.fr/livre/?GCOI=27000100308520 >.

Supports : broché

ISBN : 979-10-910281-45-4

Nombre de pages : 288

Format : $15 \times 23 \mathrm{~cm}$

Parution : février 2015

Prix : $38 €$
Supports : numérique

ISBN : 979-10-91281-46-1

Format : PDF

Parution : février 2015

Prix : $22.80 €$ 